\title{
Structural, Magnetic and Electronic \\ Properties of Nanostructured Magnetic Materials
}

By

Tushara Prakash

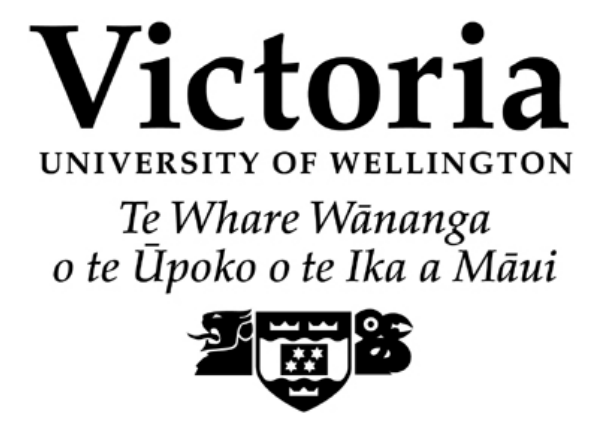

A thesis

Submitted to the Victoria University of Wellington

In fulfilment of the requirements for the degree of

Doctor of Philosophy

in Physics.

Victoria University of Wellington 


\section{Abstract}

This thesis was motivated by the different properties exhibited by magnetic nanoparticles when compared with the bulk. For example the coercivity and magnetocrystalline anisotropy vary with the particle size and the finite particle size can affect the spin-wave dispersion. When the nanoparticle radius becomes small enough it is possible to observe superparamagnetism with negligible hysteresis. The transport properties can also be different in nanoparticle composites when compared with the bulk. It is particularly interesting if the nanoparticles have a degree of electronic spin polarization because it is then possible to observe spin-dependent tunnelling. This thesis reports the results from a study of the structural, magnetic, and electronic properties of two partially electronically spin-polarized nanostructured compounds, iron-nickel alloy and magnetite, that were made using a new arcdischarge method, ion implantation and annealing, and a co-precipitation method.

It was found that permalloy powders could be made by arc-discharge where there were a range of particle sizes from nms to $10 \mathrm{~s}$ of microns. Magnetoresistance was observed where it is due to the ordinary magnetoresistance and spin-dependent tunnelling between the particles. It was also possible to make magnetite using the arcdischarge process and the powders contained nanoparticles, large faceted nanoparticles, and larger particles in the $10 \mathrm{~s}$ of micron range. The temperature dependence of the saturation magnetization changes at $127 \mathrm{~K}$, which can be attributed to the charge-ordering Verwey transition. A large magnetoresistance was observed and attributed to spin-dependent tunnelling between the magnetite particles. It was less than predicted due to a spin-disordered interfacial region. The electrical resistance was modelled in terms of small nanoparticles coating the larger particles and electrostatic charging during tunnelling between small nanoparticles. Magnetite powders were also synthesized via a chemical co-precipitation method where nanoparticles with diameters of $\sim 14 \mathrm{~nm}$ were observed. The Verwey transition was only observed in the zero-field cooled field-cooled magnetization for the arcdischarge powders. It was observed for the magnetite powders made using both methods in the temperature dependence of the saturation moment. The saturation magnetic moment for powders made using both methods has a power law dependence 
on temperature with an exponent of $3 / 2$ at low temperatures and a higher value above the Verwey transition temperature 2. There was also a large magnetoresistance due to spin-dependent tunnelling for magnetite nanoparticle made using a chemical coprecipitation method and the electrical resistance could be modelled in terms of electrostatic charging during tunnelling.

$\mathrm{Ni}_{\mathrm{x}} \mathrm{Fe}_{1-\mathrm{x}}$ nanoparticles were made for the first time by ion beam implantation. Small superparamagnetic nanoparticles occurred after implantation. The saturation moment after implantation did not follow the Bloch's $\mathrm{T}^{3 / 2}$ for $\mathrm{x}=0.82$, which is likely to be due to spin-waves propagating in the nanoparticle/ $\mathrm{Ni}_{\mathrm{y}} \mathrm{Fe}_{1-\mathrm{y}} \mathrm{Si}_{\mathrm{z}} \mathrm{O}_{\mathrm{n}}$ matrix. A bimodal particle size distribution of mostly spherical nanoparticles was observed for $\mathrm{x}=0.82$ after annealing. An $\mathrm{x}=0.45$ sample showed large asymmetric $\mathrm{Ni}_{\mathrm{x}} \mathrm{Fe}_{1-\mathrm{x}}$ nanoparticles with minimal smaller nanoparticles. The different nanoparticle morphologies is likely to be due to the different nucleation centres and the different initial concentration profiles. The saturation moment had an exponent of $3 / 2$ at low temperatures and there was a contribution from surface disordered spins. A higher $\mathrm{Ni}$ fluence with $\mathrm{x}=0.53$ lead to the formation of superparamagnetic nanoparticles that had a higher blocking temperature, indicating the formation of larger nanocrystallites. There was an enhancement in the permeability. 


\section{TABLE OF CONTENTS}

\section{INTRODUCTION}

\section{BACKGROUND AND THEORY}

6

$\begin{array}{ll}\text { HISTORICAL BACKGROUND } & 6\end{array}$

$\begin{array}{ll}\text { THEORY } & 8\end{array}$

METHODS OF PREPARATION $\quad 8$

SPIN POLARIZED MATERIALS $\quad 8$

PERMALLOY $\quad 9$

MAGNETITE

MAGNETIC ANISOTROPY 13

DEMAGNETIZATION FACTORS

MAGNETIC ORDER 16

SUPERPARAMAGNETISM

$\begin{array}{lr}\text { SPIN DISORDER } & 18\end{array}$

$\begin{array}{ll}\text { SPIN POLARIZATION } & 18\end{array}$

MAGNETORESISTANCE 20

TUNNELLING MAGNETORESISTANCE

ORDINARY MAGNETORESISTANCE 21

ANISOTROPIC MAGNETORESISTANCE $\quad 22$

MECHANISMS OF RESISTIVITY: RESISTIVITY IN METALS 23

\begin{tabular}{lr} 
3. EXPERIMENTAL DETAILS & 27 \\
\hline
\end{tabular}

$\begin{array}{lr}\text { SYNTHESIS TECHNIQUES } & 27\end{array}$

ARC DISCHARGE SYNTHESIS OF MAGNETIC POWDERS 27

CHEMICAL CO-PRECIPITATION METHOD

PRESSING AND SINTERING $\quad 30$

ION IMPLANTATION

ELECTRON BEAM ANNEALING

CHARACTERIZATION TECHNIQUES

ELEMENTAL COMPOSITION - ION BEAM ANALYSIS (IBA) 36

RUTHERFORD BACKSCATTERING SPCECTROSCOPY 36

X-RAY DIFFRACTION

SCANNING ELECTRON MICROSCOPY

TRANSMISSION ELECTRON MICROSCOPY

RAMAN CONFOCAL SPECTROSCOPY $\quad 40$

MAGNETIC PROPERTIES MEASUREMENT SYSTEM (MPMS) 41

PHYSICAL PROPERTIES MEASUREMENT SYSTEM (PPMS) 42

RESISTIVITY MEASUREMENTS $\quad 42$

$\begin{array}{ll}\text { VIBRATING SAMPLE MAGNETOMETER } & 43\end{array}$ 


\section{PERMALLOY POWDERS CONTAINING NANOSTRUCTURES}

SYNTHESIZED BY ARC-DISCHARGE

ARC DISCHARGE SYNTHESIS 46

STRUCTURAL, MAGNETIC AND MAGNETORESISTANCE MEASUREMENTS 47 X-RAY DIFFRACTION

SCANNING ELECTRON MICROSCOPY AND TUNNELLING ELECTRON MICROSCOPY 49

MAGNETIZATION MEASUREMENTS

FIELD-DEPENDENT MAGNETIZATION MEASUREMENTS

TEMPERATURE DEPENDENT MAGNETIZATION MEASUREMENTS

MAGNETORESISTANCE MEASUREMENTS

IMPROVING THE DESIGN OF THE ARC DISCHARGE SYSTEM 64

$\begin{array}{ll}\text { CONCLUSIONS } & 66\end{array}$

\section{IRON OXIDE MADE BY AN ARC-DISCHARGE METHOD AND} A CO-PRECIPITATION METHOD

IRON OXIDE NANOSTRUCTURED PARTICLES WITH A SPIN-DISORDERED REGION AT THE INTERFACE

SYNTHESIS TECHNIQUE

$\begin{array}{ll}\text { STRUCTURAL ANALYSIS } & 70\end{array}$

$\begin{array}{ll}\text { X-RAY DIFFRACTION } & 70\end{array}$

TRANSMISSION ELECTRON MICROSCOPY

SCANNING ELECTRON MICROSCOPY

RAMAN CONFOCAL SPECTROSCOPY MEASUREMENTS

MAGNETIZATION MEASUREMENTS

MAGNETORESISTANCE MEASUREMENTS

$\begin{array}{lr}\text { CONCLUSIONS } & 90\end{array}$

MAGNETITE NANOPARTICLES SYNTHESIZED BY A

CO-PRECIPITATION METHOD $\quad 92$

SYNTHESIS TECHNIQUE $\quad 92$

\begin{tabular}{lr} 
STRUCTURAL INVESTIGATION & 93 \\
\hline & 93
\end{tabular}

X-RAY DIFFRACTION

SCANNING ELECTRON MICROSCOPY AND TRANSMISSION ELECTRON MICROSCOPY 95

RAMAN SPECTROSCOPY ANALYSIS $\quad 99$

$\begin{array}{lr}\text { MAGNETIZATION MEASUREMENTS } & 104\end{array}$

MAGNETORESISTANCE MEASUREMENTS $\quad 110$

$\begin{array}{ll}\text { CONCLUSIONS } & 113\end{array}$

$\begin{array}{ll}\text { SUMMARY } & 115\end{array}$ 


\section{DUAL IMPLANTATION OF IRON AND NICKEL INTO SILICON}

DIOXIDE

IMPLANTATION OF $\mathrm{Ni}_{0.78} \mathrm{Fe}_{0.22} \mathrm{IN} \mathrm{SiO}_{2} \quad 122$

SYNTHESIS TECHNIQUE 122

$\begin{array}{lr}\text { STRUCTURAL MEASUREMENTS } & 123\end{array}$

DYNAMIC TRIM SIMULATIONS 123

$\begin{array}{ll}\text { RUTHERFORD BACKSCATTERING ANALYSIS } & 124\end{array}$

TRANSMISSION ELECTRON MICROSCOPY MEASUREMENTS 125

MAGNETIZATION MEASUREMENTS $\quad 129$

$\begin{array}{ll}\text { CONCLUSIONS } & 139\end{array}$

$\begin{array}{lr}\mathrm{NI}_{0.45} \mathrm{FE}_{0.55} \text { IMPLANTED AND ANNEALED } & 141\end{array}$

$\begin{array}{ll}\text { EXPERIMENTAL DETAILS } & 141\end{array}$

$\begin{array}{lr}\text { STRUCTURAL ANALYSIS } & 142\end{array}$

$\begin{array}{lr}\text { D-TRIM SIMULATIONS } & 142\end{array}$

$\begin{array}{ll}\text { RUTHERFORD BACKSCATTERING ANALYSIS } & 143\end{array}$

$\begin{array}{lr}\text { TRANSMISSION ELECTRON MICROSCOPY } & 145\end{array}$

$\begin{array}{lr}\text { MAGNETIZATION } & 147\end{array}$

$\begin{array}{lr}\text { CONCLUSIONS } & 156\end{array}$

HIGH-FLUENCE IRON-NICKEL NANOPARTICLES IN SILICON DIOXIDE 158

$\begin{array}{lc}\text { EXPERIMENTAL DETAILS } & 158\end{array}$

$\begin{array}{lr}\text { D-TRIM SIMULATIONS } & 158\end{array}$

$\begin{array}{ll}\text { RUTHERFORD BACKSCATTERING ANALYSIS } & 159\end{array}$

$\begin{array}{lr}\text { MAGNETIZATION MEASUREMENTS } & 160\end{array}$

$\begin{array}{ll}\text { CONCLUSIONS } & 164\end{array}$

$\begin{array}{ll}\text { SUMMARY } & 166\end{array}$

\begin{tabular}{lr} 
7. SUMMARY & 172 \\
\hline
\end{tabular}

STRUCTURAL AND VIBRATIONAL STUDIES 172

$\begin{array}{lr}\text { MAGNETIC STUDY } & 173\end{array}$

$\begin{array}{ll}\text { MAGNETOTRANSPORT STUDY } & 175\end{array}$

$\begin{array}{ll}\text { OUTLOOK } & 176\end{array}$ 


\section{List of figures}

\section{CHAPTER 2}

Figure 2.1 Examples of possible arrangements of nanoparticles.

Figure 2.2 The variation of the magnetic moment of bcc (solid points) and fcc (open circles) with composition for iron + nickel alloys. The inset shows the low nickel content in a magnified scale. (Figure reproduced from Crangle, J. and Hallam, G.C., 1963, The magnetization of face-centred cubic and body-centred cubic ion + nickel alloys. In Proceedings of the Royal Society of London A: Mathematical, Physical and Engineering Sciences (Vol. 272, No. 1348, pp. 119-132). The Royal Society.)

Figure 2.3 (a) Structure of magnetite showing the $\mathrm{Fe}^{2+}$ (red circles, $\mathrm{Fe}_{\text {tet }}$ ) and the $\mathrm{Fe}^{3+}$ (yellow circles, $\mathrm{Fe}_{\text {oct }}$ ) ions arranged (b) tetrahedrally (top) and octahedrally (bottom) coordinated with the oxygen atoms (green smaller circles). [Figure reproduced from Friák, M., Schindlmayr, A. and Scheffler, M., 2007. Ab initio study of the half-metal to metal transition in strained magnetite. New journal of physics, 9(1), p.5.]

Figure 2.4 Two ferromagnetic films separated by an insulator with the two films magnetized parallel (top) and the two films magnetized antiparallel (bottom).

Figure 2.5 Schematic two-current conduction model for ferromagnetic transition-metal alloys with $\mathrm{s}$ and $\mathrm{d}$ scattering. Figure reproduced from Malozemoff, A. P. "Anisotropic magnetoresistance of amorphous and concentrated polycrystalline iron alloys." Physical Review B 32, no. 9 (1985): 6080.

\section{CHAPTER 3}

Figure 3.1: Schematic of the arc discharge system used to synthesise powders containing nanoparticles

Figure 3.2: Flowchart for a typical chemical co-precipitation process.

Figure 3.3 A $5 \mathrm{~mm}$ diameter die made of tool steel that was used for pressing of powders.

Figure 3.4 Schematic of the ion implanter with a Penning source.

Figure 3.5 A sample held on a tungsten wire being electron beam annealed at a temperature of $1000^{\circ} \mathrm{C}$.

Figure 3.6: SEM images of a magnetic iron oxide powder in backscattered electron mode (left) and the secondary electron mode (right) 
Figure 3.7: Preparation of a thin lamellae from the implanted silicon dioxide film during FIB of a film.

Figure 3.8: Dipole response curve.

Figure 3.9: Typical arrangement of a pellet placed on a sapphire substrate and soldered on to a resistivity puck set-up for four terminal measurements.

\section{CHAPTER 4}

Figure 4.1. Upper figure: XRD data from $\mathrm{P} 45$ and $\mathrm{P} 78$ made in $\mathrm{Ar}$ or $\mathrm{N}_{2}$. The data are offset for clarity and normalized to the same (111) peak intensity. The peak from P45 $\mathrm{N}_{2}$ is fit to two Gaussians with centres at $43.7^{\circ}$ and $44.1^{\circ}$. Lower figure: Lower intensity peaks of the spectra. The asterisk denotes the magnetite or maghemite.

Figure 4.2. (a) (upper figure) SEM image of the P78 powder made in $\mathrm{N}_{2}$. (lower figure) HRTEM of P78 made in Ar and the size distribution obtained from a HRTEM image of P78 made in Ar.

Figure 4.3 (a) TEM image of an aggregated cluster of permalloy 78 nanoparticles that were synthesized in Argon. (b) SAED pattern of powders of permalloy 78 made in Ar showing the presence of some magnetite (solid rings) and a permalloy phase.

Figure 4.4. The magnetization from $\mathrm{P} 45$ made in $\mathrm{N}_{2}$ and Ar at $5 \mathrm{~K}$ (closed circles) and $300 \mathrm{~K}$ (open squares) and that of P78 made in $\mathrm{Ar}$ and $\mathrm{N}_{2}$ at $5 \mathrm{~K}$ (closed circles) and $300 \mathrm{~K}$ (open squares) made in $\mathrm{N}_{2}$. The inset shows the same data for small magnetic fields.

Figure 4.5. Zero-field cooled field-cooled curves of the magnetization from P78 made in Ar in the range from $5 \mathrm{~K}$ to $360 \mathrm{~K}$, in the presence of a field of $10 \mathrm{mT}$.

Figure 4.6. Temperature-dependent curves of the magnetization from P78 made in Ar. In the range from $5 \mathrm{~K}$ to $360 \mathrm{~K}$, in the presence of fields of $5 \mathrm{~T}$ with a fit to the Bloch.

Figure 4.7. Permeability of P78 powder made in Ar at room temperature.

Figure 4.8: $\mathrm{R}(\mathrm{T})$ for the $\mathrm{P} 78$ made in $\mathrm{N}_{2}$ in the presence of a field of $0 \mathrm{~T}$.

Figure 4.9: The magnetoresistance of the pressed pellet of permalloy powder made in $\mathrm{N}_{2}$ at the temperatures of $5 \mathrm{~K}, 50 \mathrm{~K}$ and $300 \mathrm{~K}$.

Figure 4.10. (color online) The resistance from P78 made in $\mathrm{N}_{2}$ at $5 \mathrm{~K}$ as a function of the field perpendicular and parallel to the field.

Figure 4.11: (a) The magnetoresistance from P78 made in $\mathrm{N}_{2}$ at $5 \mathrm{~K}$ for the current parallel or perpendicular to $B$. (b) The magnetoresistance from P78 made in $\mathrm{N}_{2}$ at $300 \mathrm{~K}$ where the current was perpendicular or parallel to $B$. 
Figure 4.12. (color online) The resistance from P78 made in $\mathrm{N}_{2}$ at $5 \mathrm{~K}$ as a function of the angle between the current and applied magnetic field at $8 \mathrm{~T}$. The solid curve is a fit to the data using Eq. 2.

Figure 4.13 Arc discharge schematic showing the schematic of the present design (left) and the prominent areas of the design where the particles aggregate.

\section{CHAPTER 5}

\section{Section 5.1:}

Figure 5.1.1. (colour online) XRD data from the iron oxide powder. Also shown are the Miller indices for magnetite peak. The * symbols show the hematite peaks. The small peaks at $49.0^{\circ}$ and $52.40^{\circ}$ are from $\mathrm{FeO}$ and iron, respectively. The inset shows a Lorentzian fit to a high angle peak.

Figure 5.1.2. (colour online) Williamson-Hall plot of $\beta \times \cos \theta$ vs. $\sin \theta$ for the magnetite peaks in the XRD data with a linear fit (dashed line) to the data.

Figure 5.1.3. Transmission electron microscopy images showing (a) an aggregate of different sized nanoparticles, (b) an aggregate that has larger nanoparticles with some smaller nanoparticles, (c) SAED pattern of the iron oxide powders where the scale is in d-spacings. Also shown are circles that show diffraction from magnetite (red) and hematite (green), (inset shows the pattern without the marked rings) and (d) scanning electron microscopy image of the powders showing a range of particle sizes.

Figure 5.1.4. Raman spectra on the iron oxide powders taken at different spots and showing (a) magnetite, (b) magnetite and hematite, and (c) magnetite and maghemite. The Raman peaks are identifies by the allowed Raman modes for magnetite (red), hematite (green), and maghemite (blue).

Figure 5.1.5. (a) Magnetization, $M$, a function of applied field at $5 \mathrm{~K}$ (empty circles) and 300 $\mathrm{K}$ (solid circles). Also shown is the saturation magnetization from bulk $\mathrm{Fe}_{3} \mathrm{O}_{4}$ at low temperatures (dashed line). Inset: Magnetization at $300 \mathrm{~K}$ over a smaller field range.

Figure 5.1.6. Zero-field-cooled (solid curve) and field-cooled (dashed curve) magnetization curves for the iron oxide powder at $0.5 \mathrm{mT}$ and (in inset) at $10 \mathrm{mT}$.

Figure 5.1.7. Saturation magnetization, $M_{\mathrm{s}}$, as a function of temperature at an applied field of $6 \mathrm{~T}$ (solid curve). Also shown are fits to Eq. 2.11 in the low and high temperature regions 
(dashed curves). Inset: Log plot of $\left[\mathrm{M}_{\mathrm{s}}(\mathrm{T})-\mathrm{M}_{\mathrm{s}}(0)\right] / \mathrm{M}_{\mathrm{s}}(0)$ as a function of the log of the temperature (solid curve) showing a linear dependence in the temperature range of $130 \mathrm{~K}$ to $350 \mathrm{~K}$ (dashed curve).

Figure 5.1.8. (colour online) (a) Magnetoresistance plotted against the applied magnetic field at $120 \mathrm{~K}, 150 \mathrm{~K}, 180 \mathrm{~K}$, and $300 \mathrm{~K}$ (solid curves). The arrow shows increasing temperature. Also shown are fits to the data using Equations 2.14 and 5.2 (dashed curves). Inset: Plot of the fitted degree of electronic polarization, $P$, against temperature. The solid line is a guide to the eye. (b) Plot of the low-field MR against the applied field at $300 \mathrm{~K}$ showing the MR for decreasing and increasing B.

Figure 5.1.9. Schematic diagram of the model of the grain boundary region with disordered spins and ordered spins in the bulk.

Figure 5.1.10. Plot of the electrical resistance against (a) temperature and (b) $1 / T^{0.5}$. The inset shows the model for the mechanism of tunnelling between grains dominated by conduction through the smaller nanoparticles found surrounding the larger particles and are also in close contact with other small nanoparticles.

\section{Section 5.2:}

Figure 5.2.1 XRD data from the chemical iron oxide (black line) with reference samples of magnetite (dashed line) normalized to the (311) peak intensity

Figure 5.2.2 Williamson-Hall plot for the XRD data of the (a) chemical iron oxide and (b) the magnetite reference sample. The straight lines are a guide to the eye.

Figure 5.2.3. Scanning electron microscopy image of the chemical iron oxide nanoparticles synthesized by a co-precipitation method.

Figure 5.2.4. Transmission electron microscopy images of the chemical iron oxide powder showing nanoparticles and SAED pattern of the chemical iron oxide powder with the dspacings indicated from magnetite with the corresponding Miller indices and red circles (lower left). TEM image showing clusters of nanoparticles aggregates (lower right).

Figure 5.2.5. Raman spectra of reference powders of (a) magnetite, (b) maghemite and (c) hematite that were made by a chemical synthesis process.

Figure 5.2.6. Raman spectra showing (a) magnetite with the laser spot with low power of 50 $\mu \mathrm{W}$ and using the $633 \mathrm{~nm}$ laser and (b) hematite seen after exposure of the magnetite powder to high laser power of $90 \mathrm{~mW}$ for 7 minutes. Raman spectra of (c) magnetite with the laser spot with low laser power of $0.03 \mathrm{~mW}$ using the $514 \mathrm{~nm}$ laser and inset showing that the peak 
fits to two peaks at $677 \mathrm{~cm}^{-1}$ and $717 \mathrm{~cm}^{-1}$, and (d) magnetite powder annealed in hydrogen atmosphere at $600{ }^{\circ} \mathrm{C}$.

Fig. 5.2.7. (a) Magnetization in emu/g as a function of applied field of the chemical iron oxide powder at $300 \mathrm{~K}$ (open circles) and $5 \mathrm{~K}$ (closed circles). (b) Zero-field-cooled fieldcooled curves of the iron oxide powder taken at $10 \mathrm{mT}$.

Figure 5.2.8. (a) Saturation magnetization against average temperature with the fit to the Bloch equation at low temperature and higher temperature range. (b) Plot of $[\mathrm{m}(\mathrm{T})$ $\mathrm{m}(0)] / \mathrm{m}(0)$ against temperature.

Fig 5.2.9. Plot of the magnetization at different temperatures of $300 \mathrm{~K}, 200 \mathrm{~K}, 110 \mathrm{~K}$ and $5 \mathrm{~K}$. The inset is a plot of the slopes of the field loops at different temperatures and high magnetic fields.

Fig. 5.2.10. (a) Magnetization in emu/g as a function of applied field of the synthesized magnetite powder annealed at $600^{\circ} \mathrm{C}$ at $300 \mathrm{~K}$. Inset: Magnetization at $300 \mathrm{~K}$ with a maximum applied field of $0.15 \mathrm{~T}$ showing the hysteresis. (b) Zero-field-cooled field-cooled curves of the annealed iron oxide powder taken at $10 \mathrm{mT}$.

Fig. 5.2.11. Plot of MR as a function of the field at $200 \mathrm{~K}$ and $300 \mathrm{~K}$ with the fit to Eq. 2.14 and using Eq. 5.2 (dashed line) for a chemical iron oxide powder pressed into a pellet.

Fig. 5.2.12. (a) Plot of $\operatorname{Ln}(\mathrm{R}(\mathrm{T}))$ against $1 / \sqrt{T}$ and (b) plot of the resistance against temperature for the chemical iron oxide powder.

\section{CHAPTER 6}

\section{Section 6.1:}

Figure 6.1.1. DTRIM simulations for the implantation of $\mathrm{Ni}$ into silicon dioxide at a fluence of $2 \times 10^{16}$ at. $/ \mathrm{cm}^{2}$ (filled circles) and subsequent implantation of $\mathrm{Fe}$ with a fluence of $4.5 \times 10^{15}$ at. $/ \mathrm{cm}^{2}$ (open circles) into the Ni-implanted films at $10 \mathrm{keV}$ and the resultant $\mathrm{Fe}$ fraction as a function of depth (filled squares).

Figure 6.1.2. Rutherford backscattering data for the as-implanted sample (dashed curve) and the sample annealed for 1 hour at $1000{ }^{\circ} \mathrm{C}$ (solid curve) with RUMP simulations. Inset: expanded RBS plot.

Figure 6.1.3. Cross-sectional TEM images of (a) the iron-nickel implanted film showing a uniform $\mathrm{Ni}_{x} \mathrm{Fe}_{1-x}$ layer in the $\mathrm{SiO}_{2}$ film, and small particles in the as-implanted $\mathrm{Ni}_{x} \mathrm{Fe}_{1-x}$ layer (inset) and (b) the film annealed for 1 hour at $1000{ }^{\circ} \mathrm{C}$ showing large particles at the surface as well as predominately deeper diffused smaller particles. The inset to (b) shows one of the 
smaller $8 \mathrm{~nm}$ wide particles (left) and one larger protruding particle (right). Each sample had platinum and carbon layers followed by the silicon dioxide film containing the implanted $\mathrm{Fe}$ and Ni on the thick Si substrate.

Figure 6.1.4. The moment per implanted ion against the applied magnetic field for the asimplanted film at $5 \mathrm{~K}$ (filled circles) and $300 \mathrm{~K}$ (open circles).

Figure 6.1.5. (a) The moment per implanted ion, $m_{\text {ion }}$, at $1 \mathrm{~T}$ against temperature from the asimplanted film at $1 \mathrm{~T}$ (filled circles). Also shown is a fit to the data using Eq. 6.1.1 (solid curve). (b) The zero-field-cooled (solid curve) and field-cooled (dashed curve) curves at $10 \mathrm{mT}$ for the as-implanted film.

Figure 6.1.6. The moment per implanted ion against the applied magnetic field for the film annealed for 1 hour at $1000{ }^{\circ} \mathrm{C}$ at $5 \mathrm{~K}$ (filled circles) and $300 \mathrm{~K}$ (open circles).

Figure 6.1.7. (a) The moment per implanted ion, $m_{\mathrm{ion}}$, at $1 \mathrm{~T}$ against temperature for the film annealed for 1 hour at $1000{ }^{\circ} \mathrm{C}$ (filled circles). Also shown are fits to Eq. 6.1.1 (solid curve) and Eq. 6.1.3 (dashed curve). (b) Zero-field-cooled (filled circles) and field-cooled (filled up triangles) curves at $10 \mathrm{mT}$ for the annealed film.

Figure 6.1.8. Plot of $\mu_{0} \mathrm{dM} / \mathrm{dB}$ at $5 \mathrm{~K}$ from $-0.8 \mathrm{~T}$ to $0.8 \mathrm{~T}$ for the as-implanted sample (open circles) and the sample annealed for 1 hour (filled squares).

\section{Section 6.2:}

Figure 6.2.1. DTRIM simulations for the implantation of Ni into silicon dioxide at a fluence of $2 \times 10^{16}$ at. $\mathrm{cm}^{-2}$ (filled circles) and subsequent implantation of Fe with a fluence of $2.5 \times 10^{16}$ at. $\mathrm{cm}^{-2}$ (open circles) into the Ni-implanted films at $10 \mathrm{keV}$ and the resultant $\mathrm{Fe}$ fraction as a function of depth (open squares). The average implantation depth was $15 \mathrm{~nm}$ and the maximum depth was $35 \mathrm{~nm}$ for the dual implantation.

Figure 6.2.2. Rutherford backscattering data focusing on the Fe and Ni combined peaks for the as-implanted sample (dashed curve) and the sample that has been electron-beam annealed at $1000 \mathrm{C}$ for 1 hour (solid curve). Inset: RBS data over all energies and the simulations.

Figure 6.2.3. Cross-sectional TEM images of (a) the iron-nickel implanted film showing a uniform deposited layer within the silicon dioxide layer, and small particles in the asimplanted film, (b) the film annealed for 1 hour at $1000{ }^{\circ} \mathrm{C}$ showing large particles at the surface, and (c) another part of the film showing some large nanoparticles and some smaller nanoparticles. The inset shows an image of a smaller nanoparticle at a higher resolution. (d) is elemental maps of the core/shell nanoparticle seen in (b). 
Figure 6.2.4. The moment per implanted ion against the magnetic field for the as-implanted film at $5 \mathrm{~K}$ (filled circles) and $300 \mathrm{~K}$ (open circles).

Figure 6.2.5: Plot of the (a) moment per implanted ion at $1 \mathrm{~T}$ against temperature, and (b) the zero-field-cooled (open circles) and field-cooled (filled circles) magnetization at $10 \mathrm{mT}$ from the as-implanted sample. Also shown in (a) is a fit to Eq. 6.1.3.

Figure 6.2.6: Plot of the moment per f.u. divided by the saturation moment against the applied magnetic field divided by the temperature for the as-implanted sample at $300 \mathrm{~K}$ (filled circles), $220 \mathrm{~K}$ (filled up triangles) and $180 \mathrm{~K}$ (filled circles). Also shown is a Langevin fit to the data (dashed curve).

Figure 6.2.7. The moment per implanted ion against the magnetic field for the sample that was electron-beam annealed at $1000 \mathrm{C}$ for 1 hour at $5 \mathrm{~K}$ (filled circles) and $300 \mathrm{~K}$ (open circles).

Figure 6.2.8. Plot of the (a) moment per implanted ion at $1 \mathrm{~T}$ against temperature, and (b) the zero-field-cooled (open circles) and field-cooled (filled circles) magnetization at $10 \mathrm{mT}$ from the sample electron-beam annealed at $1000 \mathrm{C}$ for 1 hour. The solid curve in (a) is a fit to Eq. 6.1.3.

Figure 6.2.9. Plot of $M / H$ for the as-implanted sample (filled squares) and the sample that was EBA at $1000 \mathrm{C}$ for 1 hour (filled circles).

\section{Section 6.2.2:}

Fig. 6.2.2.1 DTRIM simulations for the implantation of Ni into silicon dioxide at a fluence of $4 \times 10^{16}$ at. $\mathrm{cm}^{-2}$ (filled circles) and subsequent implantation of $\mathrm{Fe}$ with a fluence of $3.5 \times 10^{16}$ at. $\mathrm{cm}^{-2}$ (open circles) into the Ni-implanted films at $10 \mathrm{keV}$ and the resultant $\mathrm{Fe}$ fraction as a function of depth (open squares).

Fig. 6.2.2.2 Rutherford backscattering data for the as-implanted sample with a high fluence (solid curve) and the simulation (dashed curve).

Fig. 6.2.2.3. The moment per implanted ion against B for the film with high fluence at $300 \mathrm{~K}$ and $5 \mathrm{~K}$.

Figure 6.2.2.4. The zero-field-cooled (solid curve) and field-cooled (dashed curve) curves at $10 \mathrm{mT}$ for the film that had a higher fluence.

Figure 6.2.2.5. The susceptibilities at room temperature for the high fluence sample at $300 \mathrm{~K}$ (black closed squares) and $5 \mathrm{~K}$ (red closed circles).

Figure 6.2.2.6. The moment per implanted ion, $m_{\text {ion }}$, at $1 \mathrm{~T}$ against temperature from the sample with higher fluence. Also shown is a fit to the data using Eq. 6.1.3 (dashed curve). 


\section{List of tables}

Table 2.1 Magnetocrystalline anisotropy constants of some magnetic materials (Cullity and Graham, 2011).

Table 4.1. Lattice parameters of the P45 and P78 samples made in nitrogen and argon.

Table 4.2 Summary of the d spacing values obtained from Fig. 4.3 and the d spacings of the corresponding phases and Miller indices.

Table 4.3. Table of the saturation moment per formula unit at $5 \mathrm{~K}$, and the coercive fields at $300 \mathrm{~K}$ for P45 and P78 made in different gases. 


\section{Acknowledgments}

I would like to express my sincere gratitude to my primary supervisor Dr. Grant V. M. Williams of Victoria University of Wellington and MacDiarmid Institute for Advanced Materials and Nanotechnology for his continued support through his vast array of knowledge and encouragement throughout this project. I particularly thank him for his ability to see the best potential in everything.

I thank my secondary supervisor Dr. John V. Kennedy of GNS Science and MacDiarmid Institute for Advanced Materials and Nanotechnology for introducing me to the world of ion beams, his knowledge in materials science and his constant encouragement.

I am thankful to Sergey Rubanov of the University of Melbourne who conducted so many transmission electron microscopy studies on my samples and for showing me his lab and equipment. I was also lucky to have worked with Dr. Shen Chong who helped me with SQUID magnetometry, PPMS measurements and X-ray diffraction measurements and his expertise in sample preparation. I appreciate Dr. Simon Granville for help with the SQUID and PPMS.

I also thank members from the Materials and Air team at GNS Science for helping me learn and explore materials, techniques and methods, in particular, Dr. Jerome Leveneur for sharing his experiences with fabrication and characterization of magnetic nanoclusters, Dr. Peter P Murmu for constant support and helpful discussions, and Dr. Andreas Markwitz for guidance with ion implantation and electron beam annealing. I also thank Steve Mawsley for the design for the dies, Chris Purcell for technical assistance with the ion beam analysis, John Futter, Ed Hutchinson and Bruce Crothers.

A significant amount of work was done at VUW for Raman spectroscopy and SEM studies. I thank Olly Pantoja and Brendan Darby for assistance with Raman measurements. I thank David Flynn for guiding me through using the SEM and TEM equipment. Many thanks are due to my fellow PhD students and friends Pierre Couture and Prasanth Gupta, among others from the MacDiarmid Institute for the helpful insight and support with techniques.

The results from this thesis were a part of my three years' work based at the Materials and Air team at the National Isotope Centre, GNS Science and Victoria University of Wellington. I am particularly grateful for the access to resources at GNS Science, at Robinson Research Institute and Victoria University of Wellington. This work was funded by the MacDiarmid Institute for Advanced Materials and Nanotechnology and the Ministry of Business, Innovation and Employment (C08X01206).

Finally, I thank my parents Usha and Prakash and my grandparents for their continual encouragement and excitement. I also thank my brother Vinayak and my partner Gaurav for invaluable support. 


\section{Chapter 1}

\section{Introduction}

Magnetic nanoparticles are being actively researched because they have a wide variety of potential applications that include magnetic resonance imaging contrast agents (Banerjee et al., 2010) (Pankhurst et al., 2003), medical treatment and diagnostics (Banerjee et al., 2010), (Pankhurst et al., 2003) waste water treatment (Shen et al., 2009), high density magnetic memory (Ross, 2001) (Sun et al., 2000) (Moser et al., 2002), and magnetic field sensors (Leveneur et al., 2011) (Chen et al., 2011) (Dai et al., 2011). Nanoparticles have been made with a range of techniques that lead to nanoparticle powders or nanoparticle composites.

The nanoscale physics in magnetic nanoparticles is different when compared with the bulk. For example the magnon dispersion is known to change for small nanoparticles where the small nanoparticle size can lead to the opening of a magnon gap (Hendriksen et al., 1993) and the coercivity and magnetocrystalline anisotropy can depend on the nanoparticle size (Huber, 2005). When the nanoparticle radius becomes small enough it is possible for the thermal energy to exceed the magnetocrystalline anisotropy energy and this leads to superparamagnetism with negligible hysteresis (Cullity and Graham, 2011). This can be advantageous for magnetic sensing applications where negligible hysteresis makes it easier to measure small magnetic fields. Nanostructured composites containing magnetic nanoparticles can display magnetotransport properties not seen in the bulk (Leveneur et al., 2011) (Inoue and Maekawa, 1996) (Wang et al., 2006) (Wang et al., 2009). For example, spin tunnelling between nanoparticles in a semiconducting matrix can lead to large magnetoresistances (Inoue and Maekawa, 1996; Wang et al., 2009, 2006). 
This thesis is a study of new methods to make magnetic nanoparticles and nanostructured materials and understanding their structural, magnetic and electronic properties. A layout of the thesis is described as follows. Chapter 2 is an introduction to the theoretical subject matter dealt with in this thesis. An introduction to research into magnetic nanoparticles and the various applications and synthesis methods is given. This is followed by a description of the magnetic phenomena that are relevant to this thesis with theoretical background and some examples. The various phenomena include magnetic anisotropy, demagnetization, superparamagnetism, magnetic ordering phenomena in bulk and nanoparticles, magnon dispersion, spin disorder effects and glassy behaviour. Various origins of the magnetoresistance are explored in this thesis and the types that are relevant here are tunnelling magnetoresistance, ordinary magnetoresistance, and anisotropic magnetoresistance. A brief description of each of these is given. Conduction mechanisms are described next.

Chapter 3 deals with the synthesis techniques and analysis techniques and the experimental methods used. Synthesis techniques used to make magnetic nanoparticles are the conventional chemical co-precipitation method, the arcdischarge method, pressing of powders into pellets for resistivity measurements and low-energy ion implantation. Various techniques to study the structural characteristics include X-ray diffraction (XRD), ion beam analysis, Raman confocal microscopy, electron microscopy, scanning electron microscopy (SEM), transmission electron microscopy (TEM). The major analytical measurements on the magnetic properties and the transport properties were performed using a magnetic properties measurement systems and the physical properties measurement system and these are described in greater detail. The physical properties measurement system was used in the resistivity and the vibrating sample magnetometry modes. 
Chapter 4 deals with the synthesis and properties of permalloy powders made using a novel arc-discharge mechanism. Samples were synthesised in nitrogen and air for two different compositions of iron and nickel and the structural properties studied by XRD, SEM, TEM and Raman measurements in order to determine the composition and crystalline quality. Field dependent magnetization measurements were presented for all the samples and compared to the values expected for bulk. Temperature-dependent magnetization measurements were also performed. Temperature-dependent resistivity measurements were performed on a pressed pellet of this sample that confirmed the metallic nature of the sample. Magnetoresistance measurements at different temperatures and angle-dependent magnetoresistance measurements helped establish the origins of the magnetoresistance. The low spin-tunnelling magnetoresistance was attributable to the degradation of the spin polarization.

Chapter 5 is on magnetite powder made by the novel arc-discharge mechanism characterised by magnetic and magnetoresistance properties and iron oxide made by a chemical co-precipitation method. For each set of materials, the structural properties, constitution and crystalline quality were investigated via XRD, SEM, TEM and Raman measurements. Field-dependent measurements were performed to determine the saturation magnetization, coercivity and other quantities and compared to the expected value in bulk. Temperature-dependent magnetization measurements were performed in order to observe the Verwey transition, determine the blocking temperature and the Bloch exponent. Magnetoresistance measurements were done across various temperatures and it was modelled using a phenomenological model. Using this model, values of spin polarization were determined and the lower than expected magnetoresistance was explained using a model of a spin disordered region between the grains. 
Chapter 6 deals with the synthesis of $\mathrm{Ni}_{\mathrm{x}} \mathrm{Fe}_{1-\mathrm{x}}$ by low-energy dual implantation of iron and nickel into silicon dioxide and their structural and magnetic properties. Two $x$ values of 0.78 and 0.45 were studied with the same $\mathrm{Ni}$ fluence as well as $\mathrm{x}=0.53$ with a higher Ni fluence. Dynamic Transport of Ions in Matter (D-TRIM) simulations were used for beam energy selection and to study the depth profile. After implantation, ion beam analysis and TEM were used to determine the composition and depth profile of the implanted and electron beam annealed (EBA) film. Magnetization measurements - field dependent and temperature dependent - were used to determine the magnetization saturation values and study the magnon dispersion for both the films before and after annealing. A model was used to account for the temperature dependence of the saturation moment that included a spin-disordered shell and the thickness of the disordered layer was determined. Electron energy loss spectroscopy (EELS) was also done for the $\mathrm{x}=0.45$ sample to study the composition of core-shell particles.

Chapter 7 is a summary on the results in this thesis and a comparison of the different nanoparticles and powders as well as the future outlook. 


\section{References}

Banerjee, R., Katsenovich, Y., Lagos, L., Mclintosh, M., Zhang, X., Li, C.-Z., 2010. Nanomedicine: magnetic nanoparticles and their biomedical applications. Current medicinal chemistry 17, 3120-3141.

Chen, L.X., Huang, X.G., Zhu, J.H., Li, G.C., Lan, S., 2011. Fiber magnetic-field sensor based on nanoparticle magnetic fluid and Fresnel reflection. Optics letters 36, 2761-2763.

Cullity, B.D., Graham, C.D., 2011. Introduction to magnetic materials. John Wiley \& Sons.

Dai, J., Yang, M., Li, X., Liu, H., Tong, X., 2011. Magnetic field sensor based on magnetic fluid clad etched fiber Bragg grating. Optical Fiber Technology 17, 210-213.

Hendriksen, P.V., Linderoth, S., Lindg $\backslash$ a ard, P.-A., 1993. Finite-size modifications of the magnetic properties of clusters. Physical Review B 48, 7259.

Huber, D.L., 2005. Synthesis, properties, and applications of iron nanoparticles. Small 1 , 482-501.

Inoue, J., Maekawa, S., 1996. Theory of tunneling magnetoresistance in granular magnetic films. Physical Review B 53, R11927.

Leveneur, J., Kennedy, J., Williams, G.V.M., Metson, J., Markwitz, A., 2011. Large room temperature magnetoresistance in ion beam synthesized surface $\mathrm{Fe}$ nanoclusters on $\mathrm{SiO}_{2}$. Applied Physics Letters 98, 053111. doi:10.1063/1.3553274

Moser, A., Takano, K., Margulies, D.T., Albrecht, M., Sonobe, Y., Ikeda, Y., Sun, S., Fullerton, E.E., 2002. Magnetic recording: advancing into the future. Journal of Physics D: Applied Physics 35, R157.

Pankhurst, Q.A., Connolly, J., Jones, S.K., Dobson, J.J., 2003. Applications of magnetic nanoparticles in biomedicine. Journal of physics D: Applied physics 36, R167.

Ross, C.A., 2001. Patterned magnetic recording media. Annual Review of Materials Research 31, 203-235.

Shen, Y.F., Tang, J., Nie, Z.H., Wang, Y.D., Ren, Y., Zuo, L., 2009. Preparation and application of magnetic $\mathrm{Fe}_{3} \mathrm{O}_{4}$ nanoparticles for wastewater purification. Separation and Purification Technology 68, 312-319.

Sun, S., Murray, C.B., Weller, D., Folks, L., Moser, A., 2000. Monodisperse FePt nanoparticles and ferromagnetic FePt nanocrystal superlattices. Science 287, 1989-1992.

Wang, S., Yue, F.J., Wu, D., Zhang, F.M., Zhong, W., Du, Y.W., 2009. Enhanced magnetoresistance in self-assembled monolayer of oleic acid molecules on $\mathrm{Fe}_{3} \mathrm{O}_{4}$ nanoparticles. Applied Physics Letters 94, 012507. doi:10.1063/1.3059571

Wang, W., Yu, M., Batzill, M., He, J., Diebold, U., Tang, J., 2006. Enhanced tunneling magnetoresistance and high-spin polarization at room temperature in a polystyrene-coated $\mathrm{Fe}_{3} \mathrm{O}_{4}$ granular system. Physical Review B 73, 134412. 


\section{Chapter 2}

\section{Background and theory}

\subsection{Historical background}

The earliest nanotechnologists date back to as far as the $4^{\text {th }}$ century AD, when nanoparticles were used for paintings and sculptures (Horikoshi and Serpone, 2013) and interesting effects were observed, such as the change in colour of the material upon illumination. Metallic nanoparticles were used in medieval time for applications that included glass making where they produced vivid colours. This was described by Michael Faraday and it was called the FaradayTyndall effect (Tweney, 2006). It was only with the advent of quantum mechanics and more detailed studies that it was shown that the different colours are due to surface plasmons in metallic nanoparticles. The detailed study and synthesis of nanoparticles started late last century due to the potential applications in medicine, electronics, sun screens, antibacterial agents, etc. Nanoparticles form part of the nanotechnology area that is most well-known in the semiconductor industry when electronic elements are now reaching the 10 s of nm scale.

The definition of nanoparticles can have different interpretations across academic circles, but in general, nanoparticles can range from 1-100 $\mathrm{nm}$ and the term nanostructures largely refers to structures that have been constrained at least in one dimension to the range of nanometres, although nanoparticles can be grouped randomly, fractally or closely packed among other arrangements, as shown in Figure 2.1. Metallic nanoparticles can have interesting properties such as higher specific surface areas, specific optical properties, mechanical strengths and specific magnetizations. 

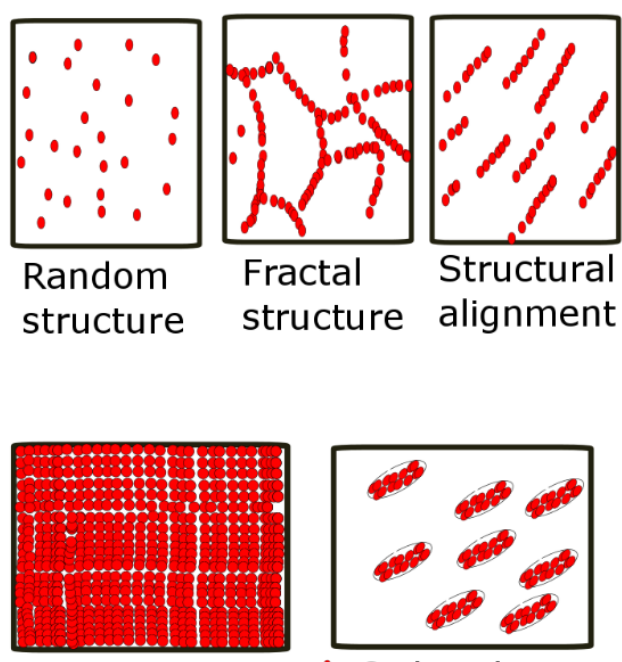

Closely packed structure

Ordered structure (dispersion)

Figure 2.1 Examples of possible arrangements of nanoparticles. 


\section{$2.2 \quad$ Theory}

\subsubsection{Methods of preparation}

Nanoparticles are made by a number of methods that include simple chemical co-precipitation methods (Aquino et al., 2005) (Maaz et al., 2010) (Wang et al., 2009), polyol processing (Qin et al., 2011), and microemulsion method (Cushing et al., 2004). Nanoparticles and nanoparticle composites are made by a number of methods like radio frequency plasma torch synthesis (Son et al., 2002), magnetron sputtering (Sun et al., 2003) and pulsed laser ablation that are being developed recently. The various methods also include ion implantation that can be used to create nanostructured magnetic materials that are embedded in silicon dioxide (Leveneur et al., 2011) (Amekura et al., 2004) (Malik et al., 2012) (Kennedy et al., 2011; Lobotka et al., 2007) (Sprouster et al., 2011) (Wang et al., 2008) (Zhu et al., 2006). The advantages of ion implantation over other methods include the ability to control to high precision the depth and concentration at a nanoscale level (Ziegler, J. F. (Ed.), 2012). It has recently been shown that that low-energy $\mathrm{Fe}$ ion implantation into $\mathrm{SiO}_{2}$ followed by EBA leads to superparamagnetic Fe nanoparticles in the surface region that have a large room temperature magnetoresistance that can be useful for magnetic sensing applications (Leveneur et al., 2011). A range of other magnetic nanoparticles have been made by ion implantation into $\mathrm{SiO}_{2}$ that includes Ni (Amekura et al., 2004; Malik et al., 2012; Sprouster et al., 2011) and Co (Malik et al., 2012) nanoparticles.

\subsubsection{Spin-polarized materials}

In this section, we look at some highly spin polarized materials that are of interest in this thesis. Spin polarization is discussed in section 2.2.7. 


\subsubsection{Permalloy}

Permalloy is a well-known metallic alloy, made of iron and nickel and some additives (e.g. $\mathrm{Cr}, \mathrm{Mo}, \mathrm{Mn}$ and $\mathrm{Cu}$ ) of lower concentration, used in industry. Of the various composition of iron-nickel alloy, permalloy $78\left(\mathrm{P} 78, \mathrm{Ni}_{0.22} \mathrm{Fe}_{0.78}\right)$ and permalloy $45\left(\mathrm{P} 45, \mathrm{Ni}_{0.45} \mathrm{Fe}_{0.55}\right)$ are face centred cubic (fcc). The phase varies from fcc to bcc as the concentration of the bcc iron is increased (Reck and Fry, 1969). Iron-nickel alloy (to be distinguished from permalloy, which contains additives) with a bcc structure varies across $x<0.30$, as is apparent in Figure 2.2. The fcc structure varies across the concentration from $x>0.30$. The Curie temperatures of fcc iron-nickel alloy and bcc iron-nickel alloy are $871 \mathrm{~K}$ and $553 \mathrm{~K}$, respectively, which is much above room temperature, which is ideal for potential room temperature applications.

The magnetic properties, including the saturation moment and the anisotropy can vary significantly according to the annealing regime that the alloy has been subjected to. Permalloy 78 has the least magneto-crystalline anisotropy in the well-annealed regime (Bozorth, 1951) and permalloy 45 has the maximum magnetization saturation. Permalloy 78 has a high Curie temperature of $1858 \mathrm{~K}$ and a low anisotropy $\left(100-300 \mathrm{~J} \mathrm{~m}^{-3}\right)$. Permalloy also has a very high permeability in the well-annealed regime. The permeability varies as the concentration of the ratios of iron and nickel change.

The saturation moment varies as the relative ratios of the iron and nickel vary. The saturation moment of bulk bcc polycrystalline iron determined by low temperature magnetization is about $2.2 \pm 0.1 \mu_{\mathrm{B}}$. The saturation moment of amorphous iron has been calculated to be $\sim 1.7 \mu_{\mathrm{B}}$ (Grinstaff et al., 1993). The lowering of the saturation magnetization can be possibly due to the presence of random anisotropy, which is the case of correlated spin glasses (Chudnovsky et al., 1986). The magnetism of nickel also varies with the degree of structural 
disorder and it has been found to decrease to as low as $0.4 \mu_{\mathrm{B}}$ (Al-Attar and Kakehashi, 1999).

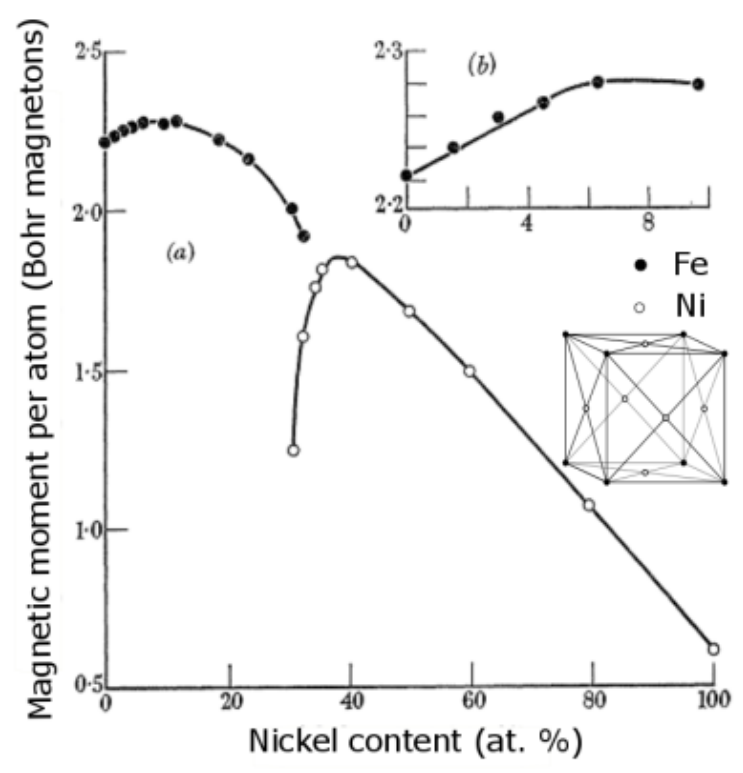

Figure 2.2 The variation of the magnetic moment of bcc (solid points) and fcc (open circles) with composition for iron-nickel alloys. The inset shows the low nickel content in a magnified scale. (Figure reproduced from Crangle, J. and Hallam, G.C., 1963, The magnetization of face-centred cubic and body-centred cubic iron-nickel alloys. In Proceedings of the Royal Society of London A: Mathematical, Physical and Engineering Sciences (Vol. 272, No. 1348, pp. 119132). The Royal Society.)

The saturation moment of the permalloy 45 is $\sim 1.8 \mu_{\mathrm{B}}$. This is near the highest saturation magnetization and the highest flux density of the permalloys. At $40 \mathrm{mT}$, the flux density is reported to be as high as $60 \mathrm{~T}$ (Elmen, 1935). The saturation moment of the permalloy 78 is $1.06 \mu_{\mathrm{B}}$. 


\subsubsection{Magnetite}

Magnetite is ferrimagnetic up to $856 \mathrm{~K}$ (Levy et al., 2012), which is ideal for room temperature applications. The crystallized structure is cubic as shown in Figure 2.3a. The $\mathrm{Fe}^{2+}$ and $\mathrm{Fe}^{3+}$ cations are arranged tetrahedrally and octahedrally coordinated with oxygen atoms, as shown in Figure 2.3b. While the oxygen atoms have small magnetic moments $\mu<0.1 \mu_{\mathrm{B}}$, where $\mu_{\mathrm{B}}$ denotes the Bohr magneton, those of the iron atoms are more than one order of magnitude larger and antiparallel on the two sub-lattices.

An interesting feature of magnetite is that it shows the Verwey transition at $\mathrm{T}_{\mathrm{V}} \sim 120 \mathrm{~K}$. It has been attributed to a charge ordering transition where the conductivity decreases by up to two orders of magnitude. It is theoretically proposed that the Verwey transition is accompanied by a change from long range charge ordering below $\mathrm{T}_{\mathrm{V}}$ to short range ordering above $\mathrm{T}_{\mathrm{V}}$. The transition can then be best described as a semiconductor-semiconductor transition with two states of different conductivities. Verwey proposed that there is a transition induced orthorhombic deformation and the ions in the $\mathrm{Fe}_{\text {oct }}$ sites becomes the $c$ axis and $\mathrm{Fe}$ ions in the cubic [110] axes perpendicular to $c$ become the orthorhombic $a$ and $b$ axes as shown in Figure 2.3c. The octahedral $\mathrm{Fe}^{3+}$ ions lie in rows parallel to $a$ and the ferrous ions parallel to $b$ 


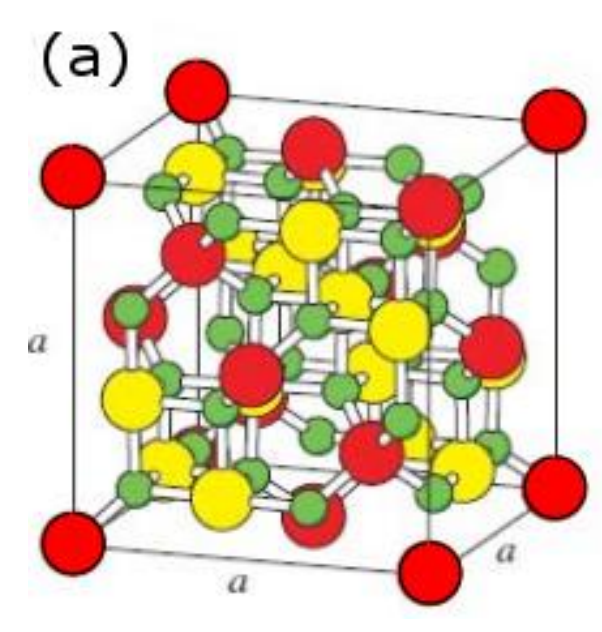

(b)

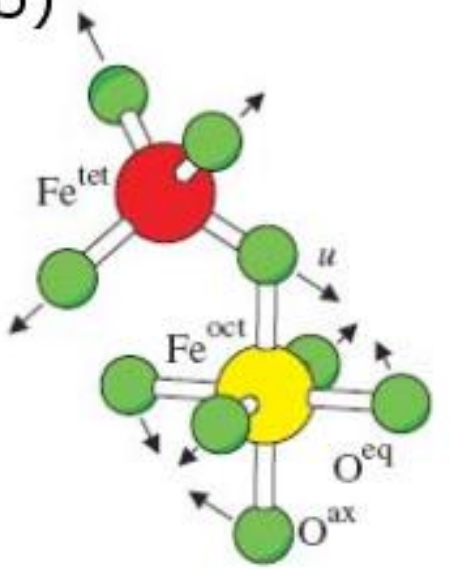

(c)

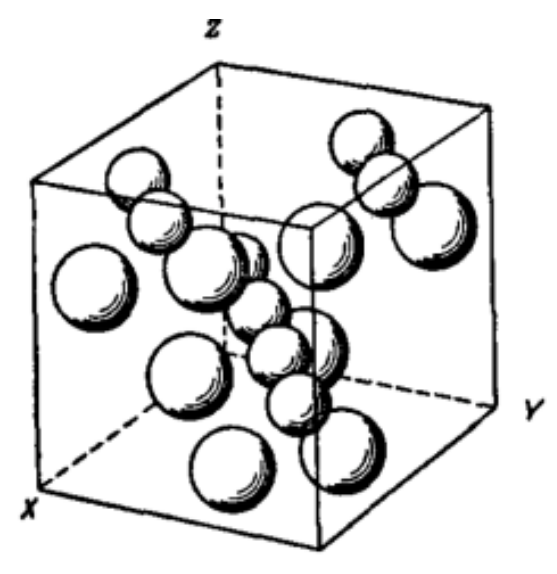

Figure 2.3 (a) Structure of magnetite showing the $\mathrm{Fe}^{2+}$ (red circles, $\mathrm{Fe}_{\mathrm{tet}}$ ) and the $\mathrm{Fe}^{3+}$ (yellow circles, $\mathrm{Fe}_{\text {oct }}$ ) ions arranged (b) tetrahedrally (top) and octahedrally (bottom) coordinated with the oxygen atoms (green smaller circles). [Figure reproduced from Friák, M., Schindlmayr, A. and Scheffler, M., 2007. Ab initio study of the half-metal to metal transition in strained magnetite. New journal of physics, 9(1), p.5.] (c) Orthorhombic structure of magnetite proposed by Verwey at $T<\mathrm{T}_{\mathrm{V}}$ [Figure from Verwey, E. J., P. W. Haayman, and F. C. Romeijn (1947), Physical properties and cation arrangements of oxides with spinel structure II. Electronic conductivity, J. Chem. Phys., 15, 181-187]. The large ions are $\mathrm{Fe}_{3+}$ and smaller ones are $\mathrm{Fe}^{2+}$. 
Later magnetoelectric studies as well as neutron diffraction studies (Mizoguchi, 1978) have shown that the low temperature structure is actually monoclinic $(C c)$ although the details of the structure is still being researched (Martín-García et al., 2016). The exchange interaction between the valence electrons and the inner electrons can cause a localization of the conduction electrons and to a partial screening of the magnetic moment (Vonsovskii, 1946) (Walz, 2002). This leads to a decrease in the saturation moment of magnetite under the Verwey transition.

\subsubsection{Magnetic anisotropy}

The intrinsic magnetic anisotropy comes from the magneto-crystalline anisotropy that arises from the spin-orbit interaction. It means that there is a preferred direction of the moments in a lattice called the easy-axis. The application of a magnetic field at 90 degrees to the easy axis is required to rotate the moments from the easy axis to this hard-axis. For cubic systems that additional contribution to the magnetic energy density can be written as,

$$
\mathrm{E}=\mathrm{K}_{0}+\mathrm{K}_{1}\left(\alpha_{1}^{2} \alpha_{2}^{2}+\alpha_{2}^{2} \alpha_{3}^{2}+\alpha_{3}^{2} \alpha_{1}^{2}\right)+\mathrm{K}_{2}\left(\alpha_{1}^{2} \alpha_{2}^{2} \alpha_{3}^{2}\right)+\ldots
$$

where $\alpha_{i}$ are direction cosines and $\mathrm{K}_{i}$ are the magneto-crystalline anisotropy constants. The important $\mathrm{K}_{i}$ are for $i>1$ and usually $\mathrm{K}_{2}$ and greater are small. For uniaxial symmetry the magnetic energy density can be written as,

$$
\mathrm{E}=\mathrm{K}_{1} \operatorname{Sin}(\vartheta)^{2}
$$

It is possible to define an anisotropy field $H_{\mathrm{K}}$ that acts on the magnetic spins that are parallel to the easy direction and of a magnitude such that for small deviations $\theta$, the torque acting on the spins can be expressed as 


$$
H_{\mathrm{K}} M_{\mathrm{S}} \sin \theta=H_{\mathrm{K}} M_{\mathrm{s}} \theta=2 K_{1} \theta
$$

where

$$
H_{\mathrm{K}}=\frac{2 K_{1}}{M_{\mathrm{S}}} \text {; }
$$

\begin{tabular}{|l|l|l|}
\hline Structure & $\boldsymbol{K}_{\mathbf{1}}\left(\times \mathbf{1 0 ^ { 4 }} \mathbf{J} / \mathbf{m}^{\mathbf{3}}\right)$ & $\boldsymbol{K}_{\mathbf{2}}\left(\times \mathbf{1 0} \mathbf{0}^{4} \mathbf{J} / \mathbf{m}^{3}\right)$ \\
\hline $\mathrm{Fe}$ & 4.8 & 0.5 \\
$\mathrm{Ni}$ & -0.5 & -0.2 \\
$\mathrm{Fe}_{3} \mathrm{O}_{4}$ & -1.1 & \\
$\mathrm{FeNi}$ & $0.03-0.04$ & 15 \\
$\mathrm{Co}$ & 45 & \\
$\mathrm{SmCo}$ & 1.8 (Sankar et al., 1975) & \\
\hline Table 2.1 Magnetocrystalline anisotropy constants of some magnetic materials \\
(Cullity and Graham, 2011) at room temperature. \\
\hline
\end{tabular}

Table 2.1 shows the magnetocrystalline anisotropy of some magnetic materials. Permalloy has a very low average magnetocrystalline anisotropy energy and this anisotropy is also found to be independent of its atomic structure and relies on the local FeNi bonding of the system that varies as the ratios of the $\mathrm{Fe}$ and $\mathrm{Ni}$ in it (Yin et al., 2006). This leads to the FeNi system being a soft magnet with vanishing anisotropy. 


\subsubsection{Demagnetization factors}

The susceptibility can be written as

$$
\chi=\frac{M}{H}
$$

where $M$ is the magnetization $(\mathrm{A} / \mathrm{m})$, and $H$ is the applied field in $\mathrm{T}$. The permeability $\mu$ is

$$
\mu=1+\chi
$$

Demagnetization occurs because there is demagnetizing field $H_{\mathrm{d}}$ in a body that can be written as

$$
H_{\mathrm{d}}=-N_{\mathrm{d}} M
$$

where $N_{\mathrm{d}}$ is the demagnetization factor or demagnetization coefficient. The value of $N_{\mathrm{d}}$ depends mainly on the shape of the body and has a calculable value only for an ellipsoid, for which it is the sum of the three orthogonal axes of an ellipsoid, and for a sphere (like in a nanoparticle), the value comes out to be

$$
N_{\text {sphere }}=\frac{1}{3}
$$

The effect of demagnetization is to stretch out the magnetization loop and this leads to a reduced measured susceptibility, $\chi_{\text {measured, }}$ that can be written as,

$$
\chi_{\text {measured }}=\chi_{\text {true }} /\left[1+\mathrm{N} \times \chi_{\text {true }}\right]
$$

where $\chi_{\text {true }}$ is the actual susceptibility in the absence of demagnetization. 


\subsubsection{Magnetic order}

Magnetic order occurs when there are exchange interactions between magnetic moments. For 3D ions that display orbital quenching the simplified Hamiltonian will include a term that can be written as

$$
H^{\text {spin }}=-J S_{1} \bullet S_{2}
$$

where $J$ is the exchange energy and $S_{1}$ and $S_{2}$ and the spins on the adjacent sites. If $J$ is positive then the spins will align parallel and this will lead to ferromagnetic order. A negative $J$ will results in the moment being antiparallel and this results in antiferromagnetic order. It is also possible to have antiferromagnetic order with moments that have different magnitudes and this leads to ferrimagnetism (e.g. as seen in magnetite and maghemite $\left.\left(\alpha-\mathrm{Fe}_{2} \mathrm{O}_{3}\right)\right)$. In this case additional magnetic terms are required in the Hamiltonian and it can be treated for example in terms of a two sub-lattice system.

For a Heisenberg ferromagnet that has a Hamiltonian as shown in Equation 2.10 it can be shown that the low wavevector magnon dispersion is quadratic and at low temperatures it can be shown that this leads to a saturation moment per ion that can be written as,

$$
m_{\text {ion, }}(\mathrm{T})=m_{\text {ion, }}(0)\left[1-B \times T^{\beta}\right]
$$

where $m_{\mathrm{ion}, \mathrm{s}}$ is the saturation moment per implanted ion, $m_{\mathrm{ion}, \mathrm{s}}(0)$ is the saturation moment per implanted ion at $0 \mathrm{~K}, B$ is a constant, and $\beta=3 / 2$. $B$ is inversely proportional to $D^{3 / 2}$ where $D$ is the spin-stiffness. Equation 2.11 with $\beta=3 / 2$ is known as Bloch's Equation. 


\subsubsection{Superparamagnetism}

When the nanoparticle radius becomes small enough it is possible for the thermal energy to exceed the magnetocrystalline anisotropy energy and this leads to superparamagnetism with negligible hysteresis (Cullity and Graham, 2011). For example, there can be assembly of uniaxial, single-domain particles, each with anisotropy energy density given by Equation 2.2. If the single-domain particles are small enough, the thermal fluctuations would be larger than the anisotropy energy in the nanoparticles and hence the moments would become randomly orientated in the absence of an applied magnetic field. It is possible to define a temperature, known as the blocking temperature, $T_{\mathrm{B}}$, where there is ferromagnetic order with the moments are in the easy-axis direction far below $T_{\mathrm{B}}$ and where they are randomly orientated with respect to the easy axis far above $T_{\mathrm{B}} . T_{\mathrm{B}}$ can be written as,

$$
T_{\mathrm{B}}=\frac{K V}{25 k_{\mathrm{B}}} .
$$

where $K$ is the effect magnetocrystalline anisotropy energy, $V$ is the nanoparticle volume, and $k_{\mathrm{B}}$ is Boltzmanns constant. For $T>T_{\mathrm{B}}$ then the magnetic moments are still ordered within a nanoparticle but the nanoparticle can be treated as having a super moment.

The materials that have been chosen for the studies are existing ferromagnetic materials with a relatively low coercivity and low magnetocrystalline anisotropy in bulk (see Table 2.1). A system such as Co-Sm and other hard magnetic materials with a large coercivity and the resulting nanoparticles that form would require a smaller size of nanoparticles to exhibit superparamagnetism. 


\subsubsection{Spin disorder}

There are a number of examples of magnetic systems where there are exchange interactions between magnetic moments but there is no long range magnetic order. It can occur for example in $\mathrm{Cu}$ doped with $\mathrm{Mn}$ where the $\mathrm{Mn}$ moments are coupled by the Ruderman-Kittel-Kasuya-Yosida interaction but the random positions mean that there are ferromagnetic and antiferromagnetic exchange. These types of systems are known as spin-glasses. Spin disorder is also known to occur in magnetic nanoparticles where there are uncompensated spins at the surface.

In a ferromagnet with random anisotropy, below a transition temperature, the spins freeze in a random orientation. This temperature is the spin glass transition temperature $T_{\mathrm{SG}}$. For an ensemble of noninteracting nanoparticles, the time needed to reverse the magnetic moment is given by $\tau=\tau_{0} \exp \left(K V / k_{B} T_{S G}\right)$, where the characteristic time constant $\tau_{0}$ is the characteristic time constant. The susceptibility can then be expressed as a function of the nanoparticles sizes $f(V)$. The behaviour of the magnetization approaching saturation can be analyzed to derive structural information. Studies indicate that the random anisotropy of materials can result in the $\mathrm{H}^{-1 / 2}, \mathrm{H}^{-1}$ or $\mathrm{H}^{-2}$ contributions at high fields (Brown, 1963).

\subsubsection{Spin polarization}

In ferromagnetic metals, electronic bands are exchange split, which implies that there are different densities of states at the Fermi energy for the upand down-spin electrons. For two ferromagnetic films separated by an insulator, the number of electrons that can tunnel through the barrier depends on the spin and consequently the tunnelling conductance depends on spin. If the films are magnetized parallel (as shown in Figure 2.4 (top)), the minority spins tunnel to the 
minority states and the majority spins tunnel through to the majority states. If however, the two films are magnetized antiparallel, the identity of the majority and minority spin electrons are reversed, so the majority spins of the first film tunnel to the minority states in the second film, and vice versa. Hence, if the spin polarizations of the two layers are very high, it is possible to have a high magnetoresistance.

Highly spin polarized materials that are ferromagnetic include magnetite, permalloy or iron-nickel alloy and iron. Magnetite, being a half metallic, is predicted to have $100 \%$ spin polarization (Zhang and Satpathy, 1991). Andreev reflection studies on permalloy have been used to determine the polarization of permalloy with a superconducting contact study reporting that the spin polarization at the point of contact is $37 \pm 5 \%$ (Soulen Jr. et al., 1998).

Spin polarization can be expressed as

$$
P=(N(\uparrow)-N(\downarrow)) /(N(\uparrow)+N(\downarrow)),
$$

where $N(\uparrow)$ is the spin-up density of states and $N(\downarrow)$ is the spin-down density of states (Žutić et al., 2004). The resulting spin-dependent TMR (Inoue and Maekawa, 1996) can be written as

$$
\mathrm{TMR}=-2 P_{1} P_{2} /\left(1-P_{1} P_{2}\right)
$$

where $P_{1}$ is the degree of electronic spin polarization in one layer and $P_{2}$ is the degree of electronic spin polarization in the other layer. 


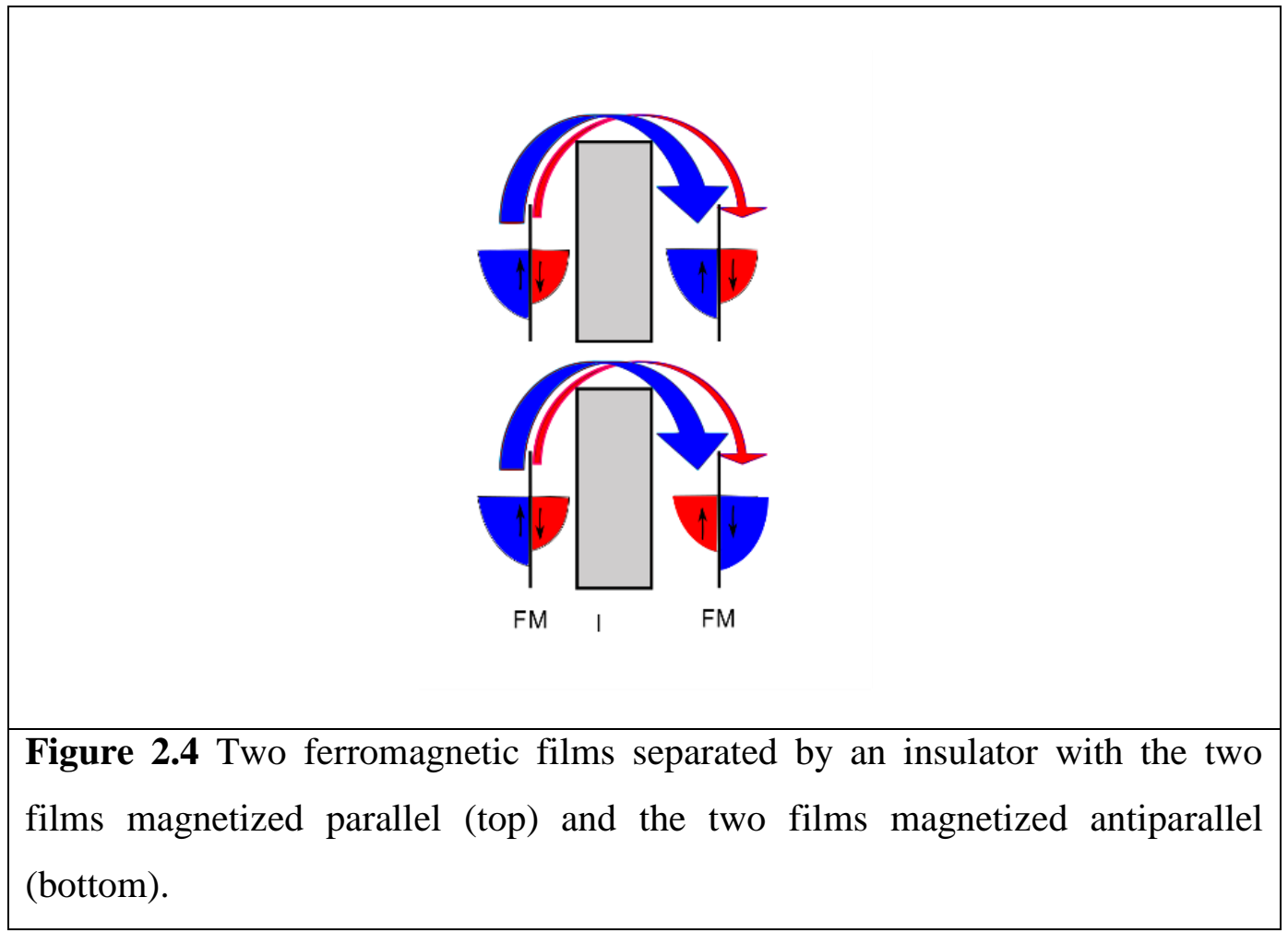

\subsubsection{Magnetoresistance}

A change in the resistivity in the material with an applied field is called the magnetoresistance (MR), which is expressed as

$$
\operatorname{MR}(B)=(R(B)-R(0)) / R(0)
$$

where $R(B)$ is the resistance for an applied magnetic flux density, $B$, and $R(0)$ is the resistance when $B=0$. There are various types of magnetoresistance and some spin dependent ones are the giant magnetoresistance, spin-dependent tunnelling magnetoresistance and anisotropic magnetoresistance. The types that are dealt with in this thesis are discussed here in brief. 


\subsubsection{Tunnelling magnetoresistance}

Tunnelling magnetoresistance occurs in materials that show a degree of electronic spin polarization as noted above. An example is magnetite has a high spin polarization that is theoretically up to $100 \%$ (Dedkov et al., 2002; Yanase and Siratori, 1984; Zhang and Satpathy, 1991) (Liu et al., 2003) (Cushing et al., 2004; LaGrow et al., 2012; Vázquez-Vázquez et al., 2011). Spin polarization studies performed using photoemission measurements show that the polarization can be as high as $80 \%$ (Dedkov et al., 2002). From Equation 2.14 is can be shown that an electronic spin polarization of $80 \%$ for tunnelling between two magnetic films with an insulating layer should result in a very high magnetoresistance of $355 \%$.

A spin-dependent tunnelling magnetoresistance can also be seen in granular systems. In this case the MR can be written as

$$
M R=-f \times m(B)^{2} /\left[1+f \times m(B)^{2}\right]
$$

where $f$ is the spin polarized fraction and $m(B)$ is the reduced magnetization. If $f=1$ then the best possible MR is $-50 \%$ when $m(\mathrm{~B})=1$.

\subsubsection{Ordinary magnetoresistance}

The ordinary magnetoresistance arises from the Lorentz force that occurs when a moving charge experiences a force, expressed by

$$
F=q(v \times B) .
$$

The additional force causes a change an increase in the path-length and hence an increased resistivity that leads to a positive magnetoresistance. 


\subsubsection{Anisotropic magnetoresistance}

Spin-orbit coupling introduces scattering between up- and down-spin $\mathrm{d}$ states with a small proportionality factor which is about 0.01 for the $3 \mathrm{~d}$ transition metals. The Campbell-Fert-Jaoul (CFJ) model has been remarkably successful in accounting for $\Delta \rho / \rho=\left(\rho_{\|}-\rho_{\perp}\right) / \rho_{\perp}$ in a permalloy and other dilute nickel-based crystalline alloys (Campbell et al., 1970) (Malozemoff, 1985). Hence, anisotropic magnetoresistance arising from spin-orbit coupling has been a subject of interest and there are various examples (Jan, 1957; Malozemoff, 1985; Mcguire and Potter, 1975; Smit, 1951; Van Gorkom et al., 2001). In the two-current conduction model schematized in Figure 2.5, conduction proceeds through two parallel channels with resistivities $\rho \uparrow$ and $\rho \downarrow$ corresponding to spin-up and spin-down conduction electrons, respectively. In the absence of spin-orbit coupling, each channel has series resistivity contributions $\rho_{\mathrm{ss}}$ arising from scattering between like spin s states and $\rho_{\mathrm{sd}}$ arising from scattering between like-spin s and $\mathrm{d}$ states; spin mixing can be ignored for high resistivity materials.

Figure 2.5 Schematic two-current conduction model for ferromagnetic
transition-metal alloys with $\mathrm{S}$ and $\mathrm{d}$ scattering. Figure reproduced from
Malozemoff, A. P. "Anisotropic magnetoresistance of amorphous and
concentrated polycrystalline iron alloys." Physical Review B 32, no. 9 (1985):
6080.




\subsubsection{Mechanisms of resistivity: resistivity in metals}

Different carrier scattering mechanisms can result in electrical resistivity that is ultimately limited by the impurity concentration. Most metals display a resistivity that is linear in temperature due to electron-phonon scattering. For strongly correlated materials it is important to also consider Coulomb interactions between electrons and this can lead to a Fermi liquid where the resistivity has a quadratic dependence on temperature.

In disordered materials it is possible to have a hopping conductivity either via localized states at the band edge or at the Fermi level. For variable range hopping at the Fermi level, the resistivity, $\rho$, can be written as

$$
\rho=\rho_{0} \times \exp \left(\left(\mathrm{T}_{0} / \mathrm{T}\right)^{\mathrm{n}}\right)
$$

where $T_{0}$ is a constant. For hopping in a $3 \mathrm{D}$ system $\mathrm{n}=1 / 4$ and for $2 \mathrm{D}$ systems this changes to $1 / 3$. In the presence of Coulomb interaction $n=1 / 2$ (Efros and Shklovskii, 1975).

\section{References}

Al-Attar, H., Kakehashi, Y., 1999. Magnetic phase diagram of Fe and Ni from crystals to amorphous structures. Journal of Applied Physics 86, 3265. doi:10.1063/1.371200

Amekura, H., Kitazawa, H., Umeda, N., Takeda, Y., Kishimoto, N., 2004. Nickel nanoparticles in silica glass fabricated by $60 \mathrm{keV}$ negative-ion implantation. Nuclear Instruments and Methods in Physics Research Section B: Beam Interactions with Materials and Atoms 222, 114-122. doi:10.1016/j.nimb.2004.01.214

Aquino, R., Depeyrot, J., Sousa, M.H., Tourinho, F.A., Dubois, E., Perzynski, R., 2005. Magnetization temperature dependence and freezing of surface spins in magnetic fluids based on ferrite nanoparticles. Physical Review B 72. doi:10.1103/PhysRevB.72.184435

Bozorth, R.M., 1951. . Ferromagnetism.

Brown, W.F., 1963. Micromagnetics Wiley. New York.

Campbell, I.A., Fert, A., Jaoul, O., 1970. The spontaneous resistivity anisotropy in $\mathrm{Ni}$ based alloys. Journal of Physics C: Solid State Physics 3, 595. 
Chudnovsky, E.M., Saslow, W.M., Serota, R.A., 1986. Ordering in ferromagnets with random anisotropy. Physical Review B 33, 251.

Cullity, B.D., Graham, C.D., 2011. Introduction to magnetic materials. John Wiley \& Sons.

Cushing, B.L., Golub, V., O'Connor, C.J., 2004. Synthesis and magnetic properties of Aucoated amorphous Fe20Ni80 nanoparticles. Journal of Physics and Chemistry of Solids 65, 825-829. doi:10.1016/j.jpcs.2003.11.027

Dedkov, Y.S., Rüdiger, U., Güntherodt, G., 2002. Evidence for the half-metallic ferromagnetic state of $\mathrm{Fe}_{3} \mathrm{O}_{4}$ by spin-resolved photoelectron spectroscopy. Physical Review B 65, 064417.

Efros, A.L., Shklovskii, B.I., 1975. Coulomb gap and low temperature conductivity of disordered systems. Journal of Physics C: Solid State Physics 8, L49.

Elmen, G., 1935. Magnetic Alloys of Iron Nickel, and Cobalt. American Institute of Electrical Engineers, Transactions of the 54, 1292-1299.

Grinstaff, M.W., Salamon, M.B., Suslick, K.S., 1993. Magnetic properties of amorphous iron. Physical Review B 48, 269.

Horikoshi, S., Serpone, N., 2013. Introduction to nanoparticles. Microwaves in Nanoparticle Synthesis: Fundamentals and Applications 1-24.

Inoue, J., Maekawa, S., 1996. Theory of tunneling magnetoresistance in granular magnetic films. Physical Review B 53, R11927.

Jan, J.-P., 1957. Galvamomagnetic and Thermomagnetic Effects in Metals, Solid State Physics - Advances in Research and Applications.

Kennedy, J., Leveneur, J., Williams, G.V.M., Mitchell, D.R.G., Markwitz, A., 2011. Fabrication of surface magnetic nanoclusters using low energy ion implantation and electron beam annealing. Nanotechnology 22, 115602. doi:10.1088/09574484/22/11/115602

LaGrow, A.P., Ingham, B., Cheong, S., Williams, G.V.M., Dotzler, C., Toney, M.F., Jefferson, D.A., Corbos, E.C., Bishop, P.T., Cookson, J., Tilley, R.D., 2012. Synthesis, Alignment, and Magnetic Properties of Monodisperse Nickel Nanocubes. Journal of the American Chemical Society 134, 855-858. doi:10.1021/ja210209r

Leveneur, J., Kennedy, J., Williams, G.V.M., Metson, J., Markwitz, A., 2011. Large room temperature magnetoresistance in ion beam synthesized surface $\mathrm{Fe}$ nanoclusters on $\mathrm{SiO}_{2}$. Applied Physics Letters 98, 053111. doi:10.1063/1.3553274

Levy, D., Giustetto, R., Hoser, A., 2012. Structure of magnetite $\left(\mathrm{Fe}_{3} \mathrm{O}_{4}\right)$ above the Curie temperature: a cation ordering study. Physics and Chemistry of Minerals 39, 169-176. doi:10.1007/s00269-011-0472-x

Liu, K., Zhao, L., Klavins, P., Osterloh, F.E., Hiramatsu, H., 2003. Extrinsic magnetoresistance in magnetite nanoparticles. Journal of Applied Physics 93, 7951-7953. doi:10.1063/1.1556133

Lobotka, P., Dérer, J., Vávra, I., de Julián Fernández, C., Mattei, G., Mazzoldi, P., 2007. Single-electron transport and magnetic properties of $\mathrm{Fe}-\mathrm{SiO}_{2}$ nanocomposites 
prepared by ion implantation. Physical Review B 75. doi:10.1103/PhysRevB.75.024423

Maaz, K., Mumtaz, A., Hasanain, S.K., Bertino, M.F., 2010. Temperature dependent coercivity and magnetization of nickel ferrite nanoparticles. Journal of Magnetism and Magnetic Materials 322, 2199-2202. doi:10.1016/j.jmmm.2010.02.010

Malik, A.E., Hutchison, W.D., Nishimura, K., Elliman, R.G., 2012. Magnetic properties of $\mathrm{Co}, \mathrm{Ni}, \mathrm{Pt}$ and their alloy nanoparticles formed in $\mathrm{SiO}_{2}$ by ion beam synthesis. Nuclear Instruments and Methods in Physics Research Section B: Beam Interactions with Materials and Atoms 272, 70-73. doi:10.1016/j.nimb.2011.01.035

Malozemoff, A.P., 1985. Anisotropic magnetoresistance of amorphous and concentrated polycrystalline iron alloys. Physical Review B 32, 6080-6083. doi:10.1103/PhysRevB.32.6080

Martín-García, L., Mascaraque, A., Pabón, B.M., Bliem, R., Parkinson, G.S., Chen, G., Schmid, A.K., de la Figuera, J., 2016. Spin reorientation transition of magnetite (001). Physical Review B 93, 134419.

Mcguire, T.R., Potter, R.I., 1975. Anisotropic Magnetoresistance in Ferromagnetic 3D Alloys. IEEE Transactions on Magnetics 11, 1018-1038. doi:10.1109/TMAG.1975.1058782

Mizoguchi, M., 1978. NMR study of the low temperature phase of Fe304. I. Experiments. Journal of the Physical Society of Japan 44, 1501-1511.

Qin, G., Pei, W., Ren, Y., Shimada, Y., Endo, Y., Yamaguchi, M., Okamoto, S., Kitakami, O., 2011. Effect of annealing on magnetic properties of Ni80Fe20 permalloy nanoparticles prepared by polyol method. Journal of nanoscience and nanotechnology 11, 10796-10799.

Reck, R.A., Fry, D.L., 1969. Orbital and spin magnetization in fe-co, fe-ni, and ni-co. Physical Review 184, 492.

Sankar, S.G., Rao, V.U.S., Segal, E., Wallace, W.E., Frederick, W.G.D., Garrett, H.J., 1975. Magnetocrystalline anisotropy of $\mathrm{SmCO}_{5}$ and its interpretation on a crystal-field model. Physical Review B 11, 435.

Smit, J., 1951. Magnetoresistance of ferromagnetic metals and alloys at low temperatures. Physica 17, 612-627.

Son, S., Taheri, M., Carpenter, E., Harris, V., McHenry, M., 2002. Synthesis of ferrite and nickel ferrite nanoparticles using radio-frequency thermal plasma torch. Journal of Applied Physics 91, 7589-7591.

Soulen Jr., R.J., Byers, J.M., Osofsky, M.S., Nadgorny, B., Ambrose, T., Cheng, S.F., Broussard, P.R., Tanaka, C.T., Nowak, J., Moodera, J.S., Barry, A., Coey, J.M.D., 1998. Measuring the spin polarization of a metal with a superconducting point contact. Science 282, 85-88. doi:10.1126/science.282.5386.85

Sprouster, D.J., Giulian, R., Araujo, L.L., Kluth, P., Johannessen, B., Kirby, N., Ridgway, M.C., 2011. Formation and structural characterization of $\mathrm{Ni}$ nanoparticles 
embedded in $\mathrm{SiO}_{2}$. Journal of Applied Physics 109, 113517. doi:10.1063/1.3594751

Sun, Y., Salamon, M., Garnier, K., Averback, R., 2003. Memory effects in an interacting magnetic nanoparticle system. Physical review letters 91, 167206.

Tweney, R.D., 2006. Discovering discovery: How Faraday found the first metallic colloid. Perspectives on Science 14, 97-121.

Van Gorkom, R.P., Caro, J., Klapwijk, T.M., Radelaar, S., 2001. Temperature and angular dependence of the anisotropic magnetoresistance in epitaxial Fe films. Physical Review B - Condensed Matter and Materials Physics 63, 1344321-1344329.

Vázquez-Vázquez, C., López-Quintela, M.A., Buján-Núñez, M.C., Rivas, J., 2011. Finite size and surface effects on the magnetic properties of cobalt ferrite nanoparticles. Journal of Nanoparticle Research 13, 1663-1676. doi:10.1007/s11051-010-99207

Wang, S., Yue, F.J., Wu, D., Zhang, F.M., Zhong, W., Du, Y.W., 2009. Enhanced magnetoresistance in self-assembled monolayer of oleic acid molecules on $\mathrm{Fe}_{3} \mathrm{O}_{4}$ nanoparticles. Applied Physics Letters 94, 2507.

Wang, Y., Qi, M., Li, G., Li, D., 2008. Morphology of large $\alpha$-Fe precipitate formed inside sapphire by ion implantation and annealing. Materials letters 62, 1444-1447.

Yanase, A., Siratori, K., 1984. Band structure in the high temperature phase of $\mathrm{Fe}_{3} \mathrm{O}_{4}$. Journal of the Physical Society of Japan 53, 312-317.

Yin, L.F., Wei, D.H., Lei, N., Zhou, L.H., Tian, C.S., Dong, G.S., Jin, X.F., Guo, L.P., Jia, Q.J., Wu, R.Q., 2006. Magnetocrystalline Anisotropy in Permalloy Revisited. Phys. Rev. Lett. 97, 067203. doi:10.1103/PhysRevLett.97.067203

Zhang, Z., Satpathy, S., 1991. Electron states, magnetism, and the Verwey transition in magnetite. Physical Review B 44, 13319.

Zhu, S., Wang, L., Zu, X., Xiang, X., 2006. Optical and magnetic properties of $\mathrm{Ni}$ nanoparticles in rutile formed by $\mathrm{Ni}$ ion implantation. Applied Physics letters 88 , 043107.

Ziegler, J. F. (Ed.), 2012. Ion Implantation Science and Technology, 2nd ed. Elsevier, Maryland, USA.

Žutić, I., Fabian, J., Sarma, S.D., 2004. Spintronics: Fundamentals and applications. Reviews of Modern Physics 76, 323. 


\section{Chapter 3}

\section{Experimental details}

This chapter discusses the methods, equipment and experimental details that were used to make nanoparticles.

\subsection{Synthesis techniques}

The different methods to synthesize the samples are explained below.

\subsubsection{Arc-discharge synthesis of magnetic powders}

Powders containing nanoparticles were synthesized using the new inhouse designed arc discharge system at GNS Science (Fang et al., 2009) (Fang et al., 2011). A schematic can be seen in Figure 3.1. Arc discharge involves a process of electrical arcing of the bulk metal to obtain powders. An arc induced from touching of the electrodes and then separating these results in a field emission of the electrons and hence a plasma arc is created. The type of plasma is determined by the atmosphere and the pressure. The temperatures formed in a plasma aids the formation of a uniform phase of material from the anode that newly forms from the bulk rod that was the anode and the cathode was Fe that sat on a water-cooled graphite disc. The cathode and part of the anode were surrounded by a metal shield that sat on the graphite disc. The anode and cathode were located inside a sealed chamber. The arc-discharge is typically carried out using a current of $74 \mathrm{~A}$ and maintaining a gap of $\sim 5 \mathrm{~mm}$ between the electrodes (Fang et al., 2014). Previous studies on Ni nanoparticles clearly show that the increase in arc current away from the optimal value would result in an increase in particles size (Förster et al., 2012). The syntheses were performed in oxygen, 
nitrogen or argon at a pressure of $\sim 400$ Torr. The powder was collected inside the base of the metal shield after arc-discharge.

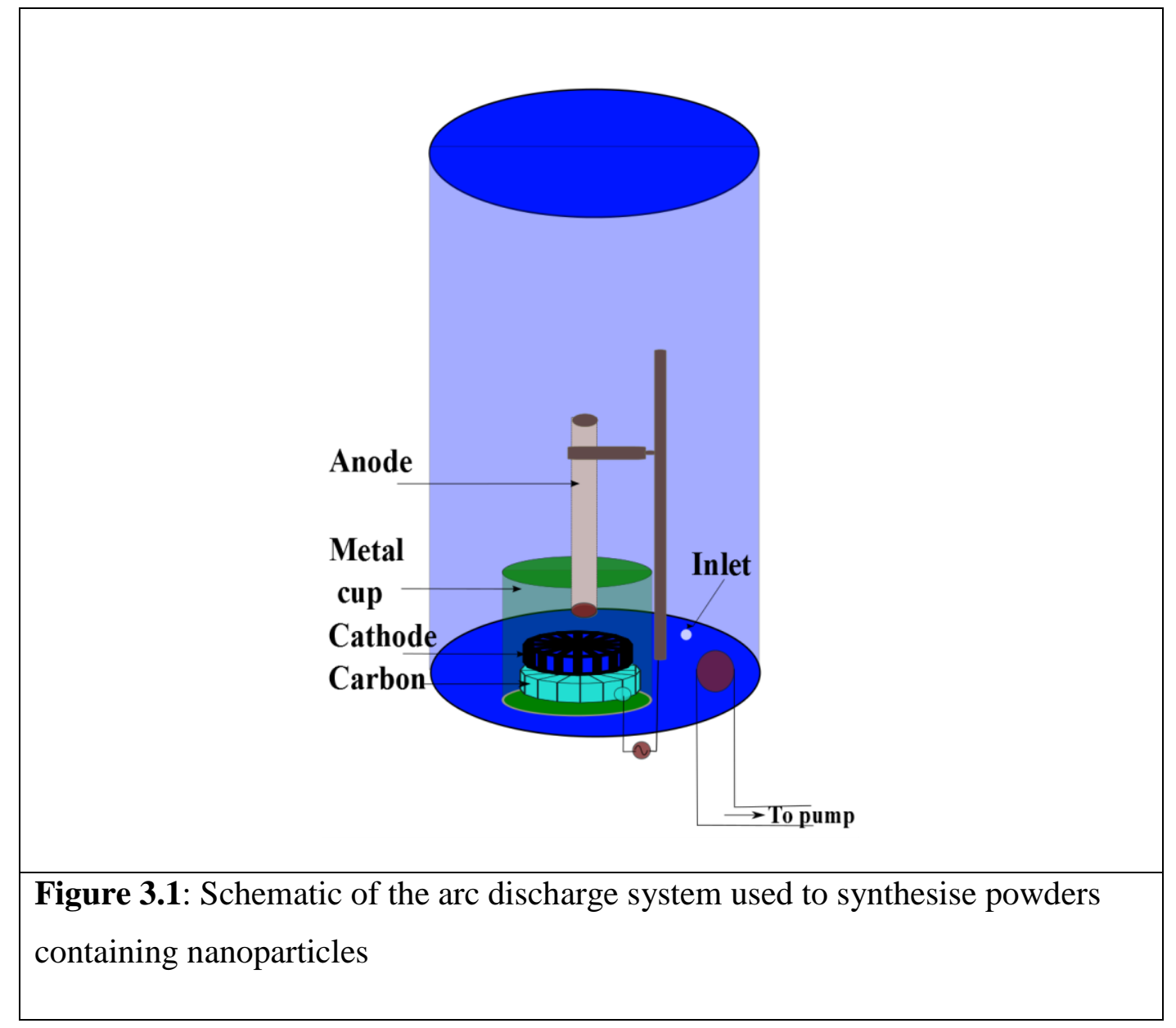

Powders have been made by other arc-discharge methods that are variations on the method used here. Powders containing Fe or Ni nanoparticles have been made using horizontal rods and a quenching solution to obtain powders that have nanoparticles with sizes from $10 \mathrm{~nm}$ to $120 \mathrm{~nm}$ (Chang and Ren, 2004). However, no scanning electron microscope measurements were made at lower magnifications to see if larger particles were present. A vertical rod assembly has been used with horizontal flow gas and a quenching region to make powders containing copper nanoparticles (Förster et al., 2012). Horizontal rods have also been used in solution and the suspended nanoparticles were obtained after the arc- 
discharge process. This process was used to make powders with bimetallic $5 \mathrm{~nm}$ $\mathrm{Ag} / \mathrm{Pt}$ nanoparticles (Pootawang et al., 2012).

Non-magnetic powders made by arc discharge include zinc oxide (Wu XuFeng et al., 2006) nanorods that grow along a particular plane, and also tungsten oxide nanostructures. Arc discharge synthesis has often been used to synthesize highly directional nanowires and nanorods, including metallic oxides (Fang et al., 2009; Yao et al., 2005) and is potentially an easier method to synthesize well aligned crystalline nanoparticles in larger quantities.

\subsubsection{Chemical co-precipitation method}

The chemical co-precipitation method (see (Pootawang et al., 2012) is a technique that is used to synthesise nanoparticles from $\mathrm{FeCl}_{2}$ and $\mathrm{FeCl}_{3}$ precursors mixed in solutions to precipitate out the salt that can be separated out to obtain nanoparticles. Precursors were mixed in beakers aided by magnetic stirrers. The mixed precursors produce a salt as well as nanoparticles that can be dissolved in solution. The solution in water is often subjected to a temperature to aid crystallisation. In this process, the nanoparticles favour nucleation over growth and Ostwald ripening leading to the formation of nanocrystallites. A flowchart of the process can be seen in Figure 3.2.

Magnetite nanoparticles synthesized by this method have been reported to vary from $2 \mathrm{~nm}$ to $11 \mathrm{~nm}$ and a saturation magnetization of $64 \mathrm{emu} / \mathrm{g}$ to $68 \mathrm{emu} / \mathrm{g}$ (Pootawang et al., 2012). The size distribution has been reported to vary with the

$\mathrm{Fe}^{2+} / \mathrm{Fe}^{3+}$ ratios of the starting materials. The particles synthesized have been reported to be polycrystalline (Pootawang et al., 2012). 

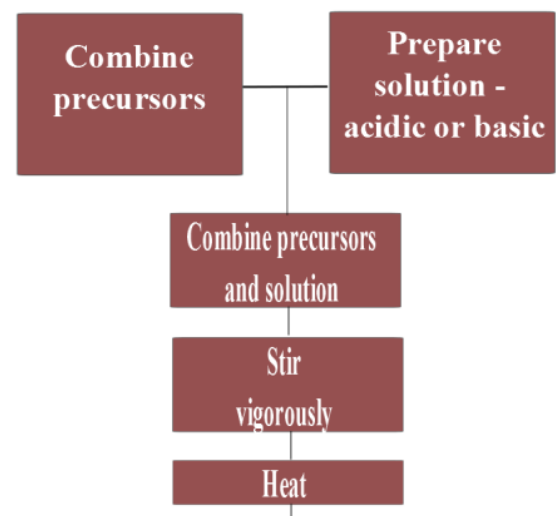

Wash the salt out

by centrifugation

Grind with isopropanol

Figure 3.2: Flowchart for a typical chemical co-precipitation process.

\subsubsection{Pressing and sintering}

Powder metallurgy techniques were adapted for this thesis in order to synthesise materials for analysis appropriate to current technologies. Pressing of the powders would enhance the density of the powder that aids conduction mechanisms and the reduction of voids. Diffusion of the particles during consolidation results in two competing mechanisms: that of microstructural coarsening and densification. Nanoparticle powders were pressed into pellets using a powder press tool in the aim of retaining the nanocrystalline structure and merely achieve densification (Chang and Zhao, 2013).

A die was designed at GNS Science for unidirectional compaction with double action or punches. Powders were packed into a die made of tool steel with diameters of either $3 \mathrm{~mm}$ or $5 \mathrm{~mm}$ and a hydraulic press was used to subject pressure onto the die containing powders. Cold pressing was adopted in order to avoid undesirable phase transformations. A typical die with $5 \mathrm{~mm}$ diameter is 
shown in Figure 3.3. The die consists of a cylinder with an opening for the lower punch as shown in the figure, a rod or an upper punch containing a holder, and a base or a lower punch. The length to thickness ratio of the dies were $\sim 3: 1$. The die is placed on top of the base, which is placed on a smooth surface.

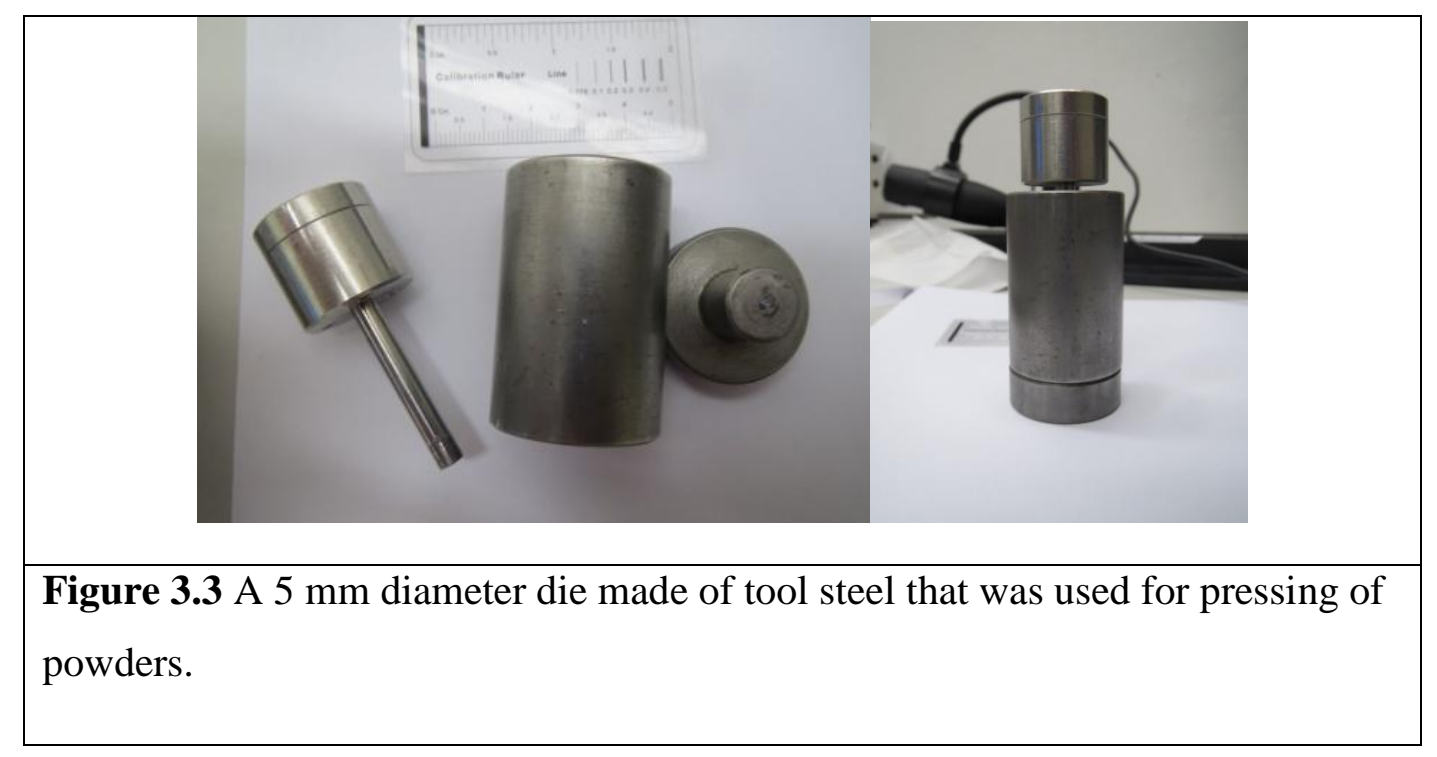

The powder is weighed and an appropriate amount is dropped into the die, tapping on the sides to ensure that the powder settles with a flat surface. The rod is then inserted into the die slowly and after full insertion, it is then turned by a few degrees to ensure that the powder is flattened and there is no un-uniformity. The design on the die allows for the removal of the pellet by removing the base creating a leeway for the rod pressing the pellet as well as space for the pellet to be pushed out. Hence, the die is then subjected to an appropriate amount of pressure. A few trials are necessary in order to achieve the required uniformity and rigidness. Next, the pressure is removed slowly. The powder should be compact and the base is removed without disturbing the rod. The rod in this particular design has been made slightly longer than the cylinder length and allows pushing out of the pellet in the same direction as the pressing. A compacted pellet appears shiny on either disc surface and does not crack or crumble when handled with a (preferably demagnetized) metallic tweezer. 
Studies on nanostructured $\mathrm{TiO}_{2}$ and $\mathrm{Y}_{2} \mathrm{O}_{3}$ powders reported an increase in the surface area density after pressing of unagglomerated spherical particles of size 7-10 nm (Skandan, $G$ et al., 1991). A review on studies that have been carried out on the sintering temperature of the compacted material reports a range of ratios of temperatures chosen for a material with respective to the melting point. This ratio ranges from 0.35 to 0.52 . However, studies on iron nanoparticles $25 \mathrm{~nm}$ wide have shown that the particles in vacuum are extremely sensitive to oxidation during sintering.

\subsubsection{Ion implantation}

Ion implantation techniques are widely used in the semiconductor industry to dope silicon and germanium films. It allows to dope a precise concentration at a desired depth (Ziegler, J. F. (Ed.), 2012). It is a versatile technique using which virtually any element can be implanted into any other element in the periodic table can be doped into the materials. The penetration depth of the ions is controlled by the energy and the concentration by the fluence (number of atoms per unit area). Low energy ion implantation enables the formation of particles at the surface (Leveneur et al., 2011). Assisted by EBA, ion implantation can be used to create nanoparticles on the surface. Implantation with large fluences has been arguably known to create nanocrystalline particles 


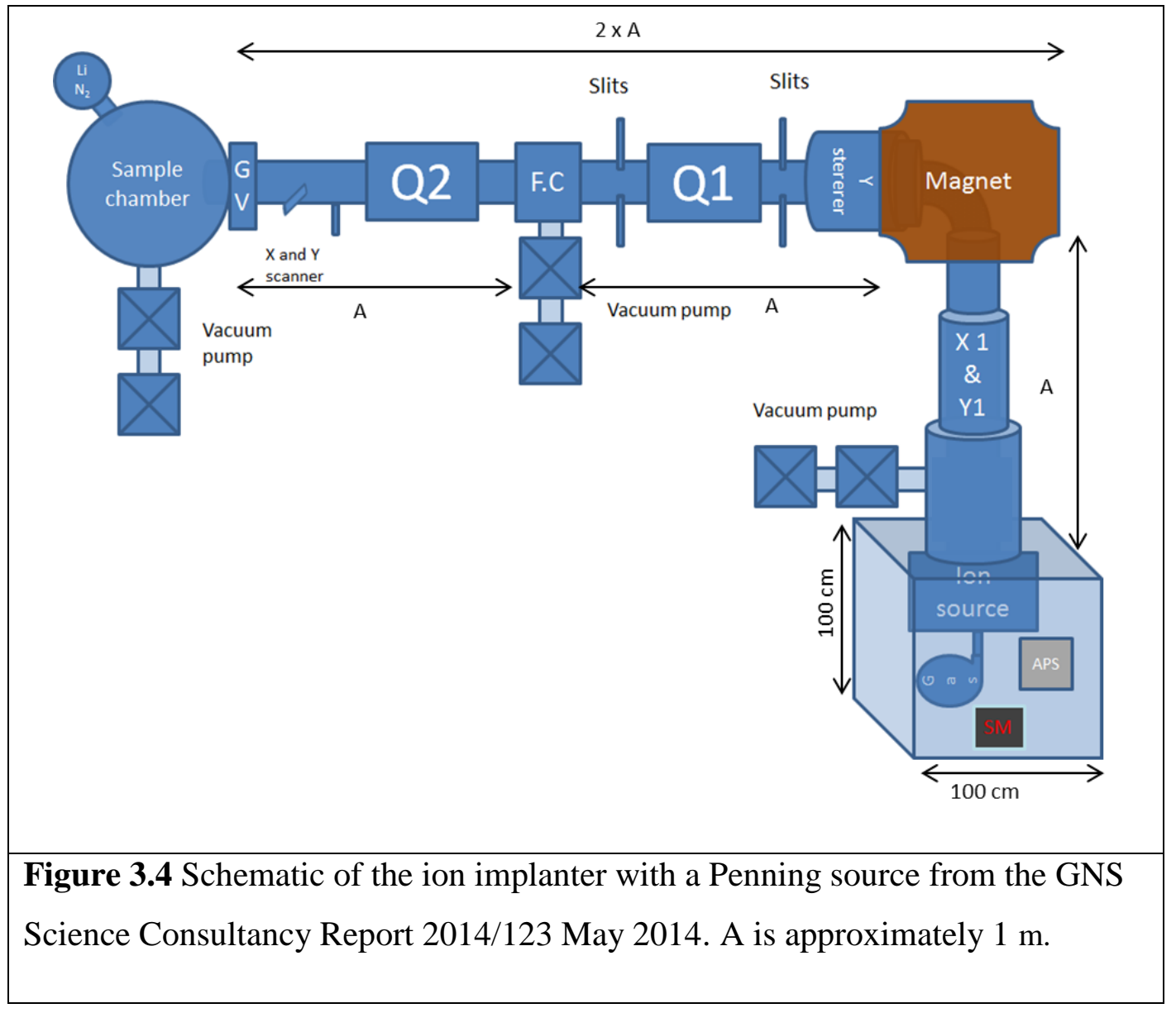

The ion implanter used for this study is the MARK-3 implanter at GNS Science (Figure 3.4). It has a range of 5-40 keV for single charge ions. It includes a Penning ion source that consists of an anode, a metal cathode with an aperture to allow ions to exit, metal ion source targets and a gas inlet and outlet. An arc creates the plasma which is confined between the electrodes with help of a magnetic field along the axis of the ion source. The plasma produced between the electrodes and the anode voltage potential accelerates the plasma towards the cathodes in order to induce sputtering. The spiralling electrons in the magnetic field increase the ionization resulting in an enhanced sputtering process. A high terminal voltage accelerates these ions through electrostatic focussing lenses toward an isotopically sensitive magnet for mass separation. The ions that get through the slit are only of the selected species. The Y steerer assists in steering 
the beam and quadrupole lenses (Q1 and Q2 in Figure 3.4) held at a high potential are in place to focus and steer the beam onto the target that is encased in a viewing chamber. A Faraday cup (F.C. in Figure 3.4) placed before the target allows for measurement of the current. Scanners in the beamline leading to the target are present to allow for raster scanning of the beam across the target for uniform intensity of the beam across the sample. The current at the target is also measured using the current integrator. This helps for a precise determination of the fluence used via a charge integrator that is connected to an automated system that allows to shut the beam off from the target when the desired fluence level is achieved. The beamline and the source are under a vacuum level of $1 \mathrm{E}-7 \mathrm{mbar}$ and the target is at $1 \mathrm{E}-6$ mbar.

In this study, iron and nickel were used as sputtering targets in the source with neon gas to create plasma. Clean cut wafer substrates that were washed in DI water containing 2\% Decon 90 and subsequently repeatedly washed with isopropanol in a sonication bath were used for implantation. The substrates were cut into a size that was larger than the implantation area. Dual implantation was carried out one implantation after another, changing the source targets in the ion source. The substrates were marked with the implanted area of the first ion, the substrate removed, then the ion source was changed and the scanning adjusted to match the first one. The previously implanted substrate was placed back at the target ready to be implanted. The dual implantation resulted in a noticeable change in the reflectivity of the substrate. This was done for uniform implantation of the resultant dual implantation.

Monte Carlo simulations using a binary collision approximation method are used to model the depth and concentration profile during implantation. In the binary collision approximation method, a screened Coulomb potential is used to model the nuclear collisions. Electron energy loss due to interaction with matter is modelled using the nuclear stopping cross section. DYNAMIC TRIM is the set of programs that calculates the stopping range and damage profiles upon 
implantation using these simulations. The main disadvantage of this method in the low energy range is that it disregards the defect clustering of atoms. TRIM takes into account the change in the composition and density of the atoms in each depth channel, according to the transport of matter by recoils and ions.

\subsubsection{Electron beam annealing}

The EBA was done at GNS Science (Kennedy et al., 2011). Annealing of samples is possible with a temperature gradient while heating as well as cooling of the sample. The beamline consists of an electron gun to generate electrons, a Wehnelt cylinder that is used to control the current that is biased at a negative voltage from the cathode, Einzel lens and a two-directional scanner (see Figure 3.5). The voltage and current of the beam, and the electron gun current can be measured. A tungsten cathode is used to generate electrons and the bias voltage is used to control the beam current. The temperature control is allowed by a thermopile for the lower temperatures and a two-colour pyrometer for higher temperatures attached to a proportional-integral-derivative controller. This allows for accurate measurement of the temperature and also allows precise control over the temperature.

EBA has been used after ion implantation and it has been known to result in the diffusion of the particles and etching of the silicon dioxide. The substrates were held in a chamber that is evacuated to $10^{-7}$ mbar with a liquid nitrogen trap. The region of interest was aligned with the region of the electron beam with the help of a laser pointer. The film sample to be annealed was not more than $4 \mathrm{~mm} *$ $4 \mathrm{~mm}$ in order to maintain a uniform temperature gradient throughout the film. A blank silicon substrate of similar area was tested in order to reach the required temperature and the uniformity of the beam. The sample was then annealed to obtain a film that was kept in desiccated chambers. 


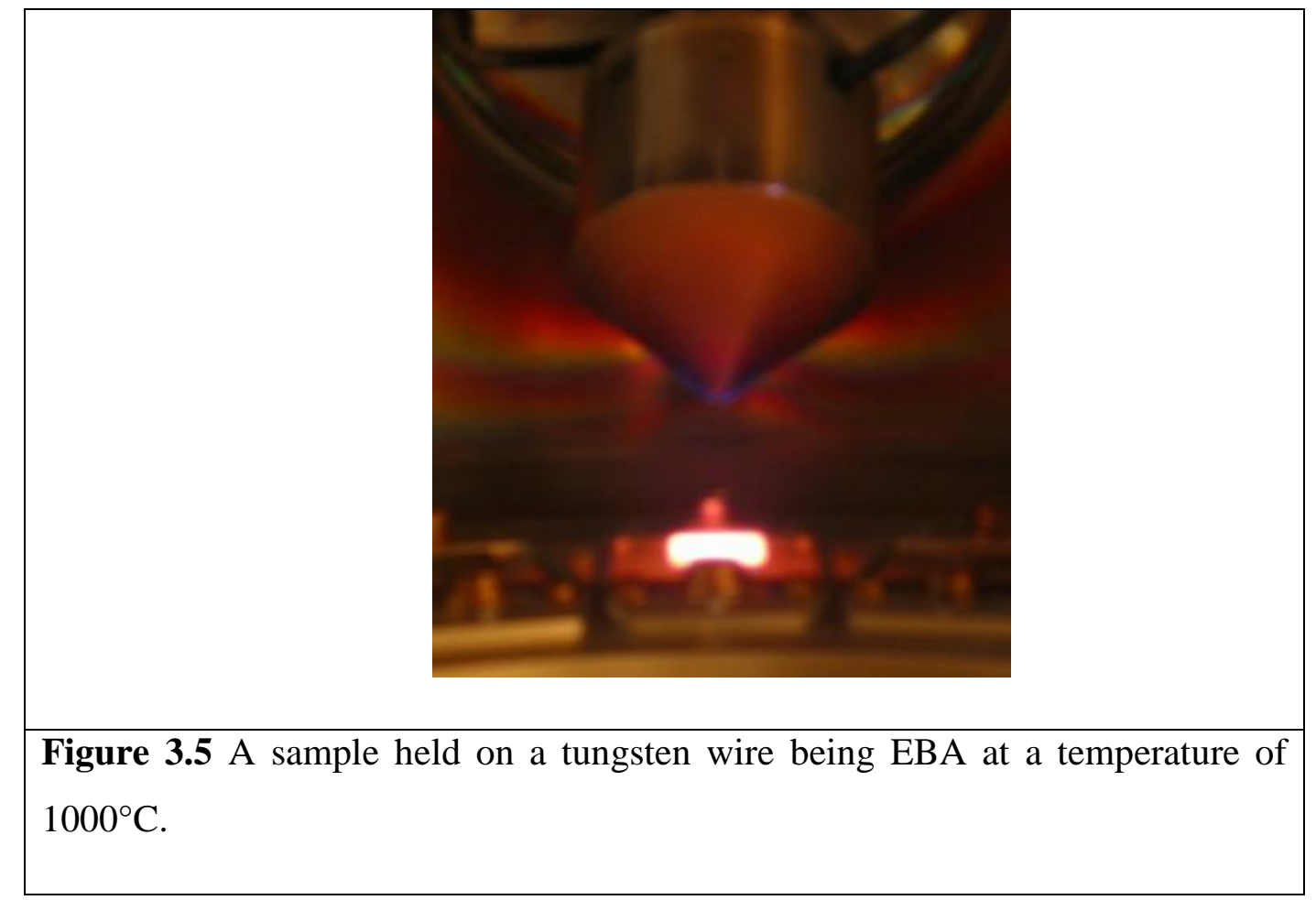

\subsection{Characterization techniques}

\subsubsection{Elemental Composition - Ion beam analysis (IBA)}

Ion beam analysis techniques were used in this thesis to analyse elemental composition of materials using particle-induced X-ray emission and the surface profile using Rutherford backscattering spectroscopy (RBS). Ion beam analysis (IBA) techniques use energetic ion beams to detect backscattered particles from the material or the X-rays emitted from the materials as a result of excitation of atoms. At GNS Science, the beamline provides for simultaneous particle-induced X-ray emission and RBS detection.

\subsubsection{Rutherford backscattering spectroscopy}

In RBS, the backscattered ions are detected in order to obtain the composition and depth profiles of a sample. Elastic collisions between the incident beam and the stationary nucleus of the sample leads to an energy loss that is relative to the scattering cross sections of the nucleus of the sample. The RBS 
set-up includes a high energy ion beam $\left(4 \mathrm{He}^{2+}\right.$ ions in this case), a surface barrier detector that was positioned at $165^{\circ}$. The high potential required for the accelerations is generated by a Van de Graff generator. The ion beam generated is guided by a set of electromagnetic systems that include focussing magnets and steerers. The solid angle of the experiment was set to $0.075 \mathrm{msr}$. The samples were mounted on a stepper motor stage and the entire process of beam exposure, positioning of the sample and collection of the data was automated. For each ion detected $(\mathrm{N}(\mathrm{E}))$, a count is produced and amplified by electronics that are then received by an analog to digital converter. The spectrum is calibrated using a silicon nitride film prior to the experiment. A typical charge amount collected during an experiment is $\sim 40 \mu \mathrm{C}$.

A well-established library of scattering cross-sections and recoil energies for elements of different masses exists. These can be used to simulate RBS spectrum by modelling multiple layers comprised of given compositions and thicknesses. In practice, this model is refined until the best fit is obtained where the simulated spectrum most closely matches the experimental spectrum. In this study the RBS simulation program RUMP (Thompson, n.d.) was used to find the desired values.

\subsubsection{X-ray Diffraction}

XRD was used to determine sample phase and crystalline quality. Constructive interference of X-rays passing through a crystalline sample can be used to obtain information on the lattice spacing of the sample and irregularity in the lattice planes. Waves that interfere constructively can be used to determine the lattice spacing through Bragg's law

$$
2 d \sin \theta=n \lambda,
$$

where $d$ is the spacing between diffracting planes, $\theta$ is the incident angle, $n$ is the diffraction order, and $\lambda$ is the X-ray wavelength of the beam. 
XRD was performed on the powder samples by pressing them flat onto an Al powder holder that held the powder in a plastic disc. The wavelengths used were $\mathrm{Cu} \mathrm{K} \mathrm{K}_{\alpha}$ radiation from copper and $\mathrm{Co} \mathrm{K}_{\alpha}$ radiation from cobalt tubes. The XRD of the powder samples were used for phase identification, average crystallite determination, any preferential grain orientation, relative amounts of the multicomponent powders and the relative amounts of amorphous material. The XRD was compared to XRD spectra of the bulk, which had narrow widths compared to that of the nanoparticles. For every given reflection, the finite size of the domains over which the diffraction is coherent can affect the breadth of the diffraction peaks. The XRD spectra that were obtained were corrected for the baseline, instrumental broadening and the $\mathrm{K} \alpha$ component.

\subsubsection{Scanning electron microscopy}

SEM was used to image the surface profile and the contrast and dimensionality of the nanoparticles and powders. This was useful in obtaining a particle size distribution and a surface profile of the samples. Because of the limits on the methods, we adopted the study of the finer particles using the TEM and focused on these in order to determine the phase and quality of the materials. The larger particles were understood through the SEM imaging.

Samples were loaded into the chamber in a low vacuum mode. In the JEOL JSM 6610F SEM at Victoria University, backscattered electron mode (BSE) as well as low energy secondary electron (SE) mode is possible. The areas with different compositions can be detected due to differences in the BSE emitted by different elements. To obtain a surface topography, the SE emitter from the kshell of the sample atoms can be detected by inelastic scattering of beam electrons. Typical SEM images can be seen in Figure 3.6. Note that for the SEM study, the same particles as was used for the TEM were used, but without dispersion and use of a lower magnification mode meant that microparticles were seen. 


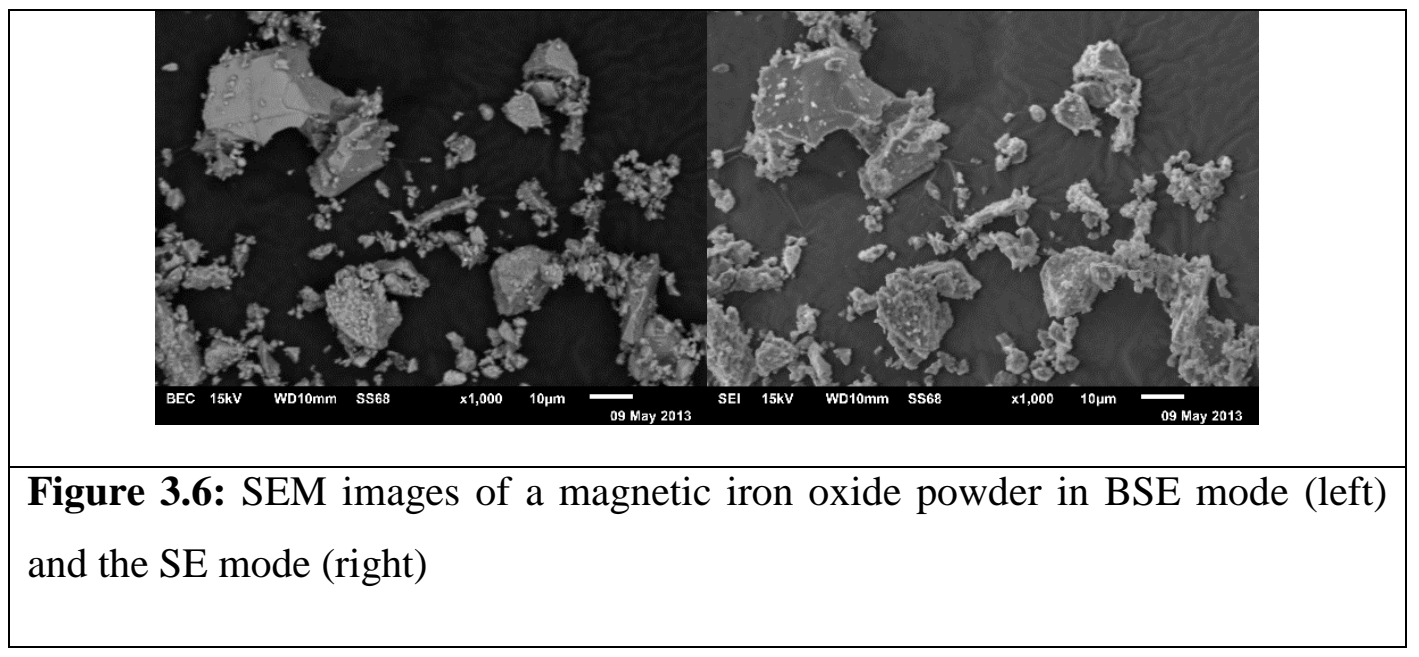

\subsubsection{Transmission electron microscopy}

The particles and films were imaged in transmission mode in order to study the high resolution, detect the presence of nanoparticles, survey features like the crystallinity and shape and detect lattice planes of samples. The TEM and EELS measurements were done at the University of Melbourne. The TEM uses electrons rather than light and thus allows viewing of particles in a nanometre scale. The de Broglie wavelength of an electron accelerated to $100 \mathrm{~s} \mathrm{of} \mathrm{keV}$ is $\sim 10-12 \mathrm{~m}$. A typical energy of the TEM is $200 \mathrm{keV}$ and the resolution possible is thus $\sim 0.16 \mathrm{~nm}$ (Used $0.753 /\left(\mathrm{aV}^{\wedge} 1 / 2\right)$; $\mathrm{V}$ is $200 \mathrm{kV}$, a is about $0.01 \mathrm{~A}$ for TEMs. The value obtained is in $\mathrm{nm}$ ). The structure of nanometre-sized regions within the film can be examined and the structure correlated with the diffraction pattern. The thin sample edge was coated with a protective layer of $\sim 100 \mathrm{~nm}$ of $\mathrm{Pt}$, and each side of the sample was thinned using a focussed ion beam (FIB) of Ga+ ions. The remaining sample was $\sim 100 \mathrm{~nm}$ thick. Figure 3.7 is a FIB image of a typical thin film being prepared by FIB. Large trenches are sputtered on either side of the area of interest. The thin membrane is thinned and lifted out. 


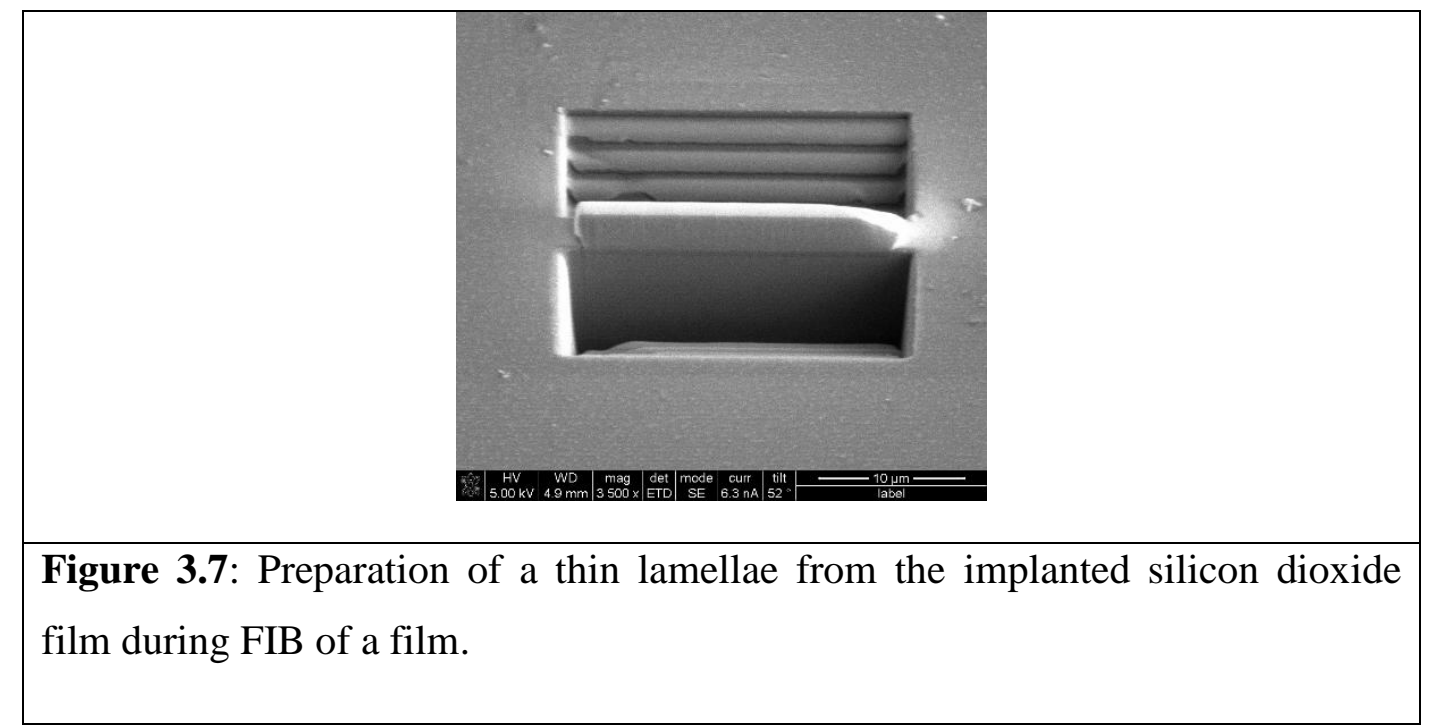

The TEM was carried out on the finer ground particles as is required for the TEM study. These particles were dispersed in the matrix and this allowed imaging of the finer nanoparticles that was the focus of the study. It was also possible to obtain selected area diffraction patterns of powder and film samples, although the lattice planes of different oxides of iron and nickel as well as the lattice planes of iron and nickel are quite different.

\subsubsection{Raman confocal spectroscopy}

Raman spectroscopy is a method to probe the phonons in a material. The light is incident on the material and there is an inelastic interaction that leads to energy loss by phonon emission (Stokes) or energy gain by phonon absorption (anti-Stokes). This can be written as $E_{\mathrm{f}}=E_{\mathrm{i}} \pm E_{\mathrm{p}}$ where $E_{\mathrm{i}}$ is the incident phonon energy, $E_{\mathrm{f}}$ is the final phonon energy and $E_{\mathrm{p}}$ is the phonon energy. Raman measurements were made using a LabRam confocal Raman microscope with a $\mathrm{HeNe}$ laser or the laser lines from an Ar ion laser. The objective magnification could reach $\times 100$ and this results in a spot size in the order or microns. 


\subsubsection{Magnetic properties measurement system (MPMS)}

Magnetization measurements were performed on a Quantum Design magnetic properties measurement system. Measurements were performed on the synthesized powders as well as the films. The superconducting quantum interference device (SQUID) magnetometer consists of a superconducting magnet, a superconducting detection coil and a SQUID connected to the detection coil. A voltage flux is induced in the pick-up coils in the presence of a magnetic moment. This voltage is transferred to the SQUID. A SQUID sensor is composed of superconducting loops that are separated by a Josephson junction. Changes in the magnetic flux in the loop induce the formation of a current in the ring. The sample is moved back and forth over a fixed scan length, and stopped at a number of specified points. At each stop, several readings of the SQUID voltage are collected and averaged. The complete scans are repeated a number of times and the signal averaged. A linear drift is calculated in a detrend program and is subtracted from each data point. The output signal obtained is analyzed by an iterative regression algorithm. This can accommodate of position offsets (up to $\pm 5 \mathrm{~mm}$ ) using an additional correction term to the calculated moment.

Finer sieved particles from the powder samples were used for the magnetization as well as the magnetoresistance studies. Powder samples were weighed and loaded on a powder capsule holder that was sealed with a hot wire. The samples, often those with a high moment, are sometimes additionally densely packed with Teflon or PTFE tape. Initial measurements on the loosely packed powders resulted in a false indication of non-saturation of the magnetic moment at high fields. This is brought about by the signal from samples moving around giving a false centre that exceeds the threshold of iterative regression. The program proceeds with the measurement using a linear regression method that assumes a linear background and applies a correction to the moment that leads to a slope in the corrected moment value that is apparent linear to the applied field. A typical dipole response curve can be seen in Figure 3.8. 


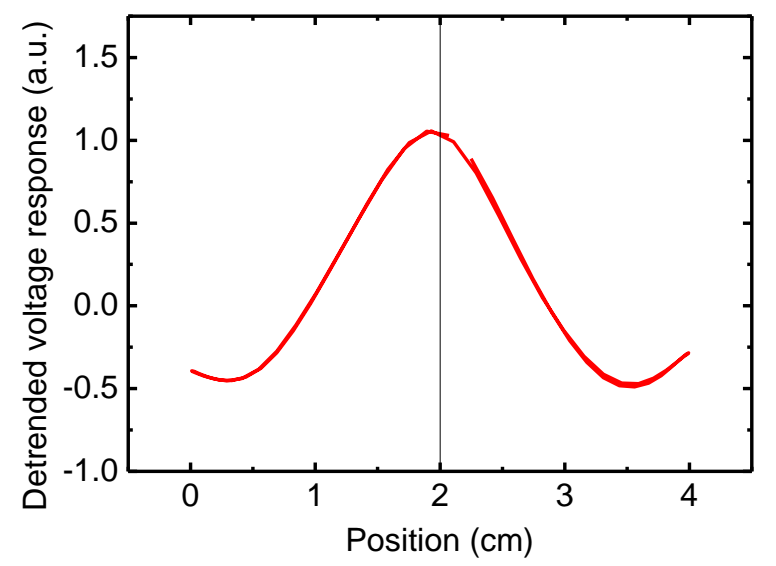

Figure 3.8: Dipole response curve from the Quantum Design magnetic properties measurement system.

\subsubsection{Physical properties measurement system (PPMS)}

\subsubsection{Resistivity measurements}

The PPMS was used for resistivity and magnetoresistance measurements. The Quantum Design PPMS was capable for the field of $+/-9 \mathrm{~T}$ and the current range from $0.01 \mu \mathrm{A}$ to $5000 \mu \mathrm{A}$. The voltage range was from 1 to $95 \mathrm{mV}$. The resistivity option required a puck holder that could hold a pellet or a film on a substrate and was held on a plastic holder with welded metal contacts that the sample could be wired to. Contacts on the samples were prepared using a multimetal evaporator. Two terminal electrical resistance measurements were used for samples that were highly resistive and four terminal measurements were used to measure samples with low resistivity. Measurements can be made with the current at different angles to the applied magnetic field. Temperature measurements from $2 \mathrm{~K}$ to $400 \mathrm{~K}$ are possible. Figure 3.9 shows a sample on a resistivity puck. 


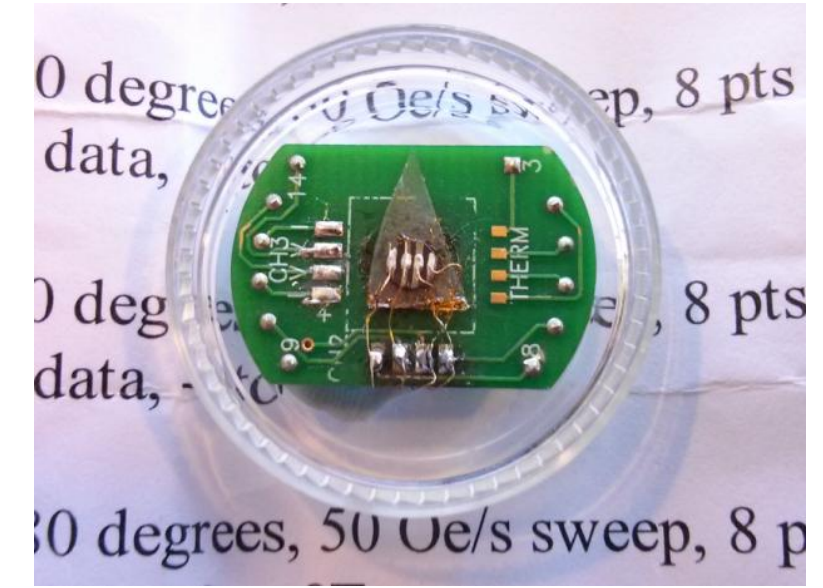

Figure 3.9: Typical arrangement of a pellet placed on a sapphire substrate and soldered on to a resistivity puck set-up for four terminal measurements.

\subsubsection{Vibrating sample magnetometer}

A vibrating sample magnetometer was used for some powder samples, the advantage being the higher limit on the maximum moment that can be measured due to the measurement technique being that of the detection of the voltage as it is passed through a coil. The sample can be arranged so that the powder sits at the right position and then vibrates. The voltage induced in the coil is detected by a gradiometer pickup coil configuration. The centre of oscillation of the sample is positioned at the vertical centre of the pickup coil. An optical linear encoder signal read back controls the alignment and the setup of the amplitude of the oscillation. The detected voltage and the position are used to generate a complete and independent magnetization measurement every cycle of oscillation and these data are averaged over a time that is specified by the user. 


\section{References}

Chang, I.T.H., Ren, Z., 2004. Simple processing method and characterisation of nanosized metal powders. Materials Science and Engineering: A 375-377, 66-71. doi:10.1016/j.msea.2003.10.005

Chang, I., Zhao, Y., 2013. Advances in powder metallurgy: Properties, processing and applications. Elsevier.

Fang, F., Futter, J., Markwitz, A., Kennedy, J., 2009. UV and humidity sensing properties of $\mathrm{ZnO}$ nanorods prepared by the arc discharge method. Nanotechnology 20, 245502. doi:10.1088/0957-4484/20/24/245502

Fang, F., Kennedy, J., Carder, D., Futter, J., Rubanov, S., 2014. Investigations of near infrared reflective behaviour of TiO 2 nanopowders synthesized by arc discharge. Optical Materials 36, 1260-1265.

Fang, F., Kennedy, J.V., Manikandan, E., Futter, R.J., Markwitz, A., 2011. Morphology and characterization of $\mathrm{TiO}_{2}$ nanoparticles synthesized by arc discharge. Chem. Phys. Lett. 521, 86.

Förster, H., Wolfrum, C., Peukert, W., 2012. Experimental study of metal nanoparticle synthesis by an arc evaporation/condensation process. Journal of Nanoparticle Research 14. doi:10.1007/s11051-012-0926-1

Kennedy, J., Leveneur, J., Williams, G.V.M., Mitchell, D.R.G., Markwitz, A., 2011. Fabrication of surface magnetic nanoclusters using low energy ion implantation and electron beam annealing. Nanotechnology 22, 115602. doi:10.1088/0957$4484 / 22 / 11 / 115602$

Leveneur, J., Kennedy, J., Williams, G.V.M., Metson, J., Markwitz, A., 2011. Large room temperature magnetoresistance in ion beam synthesized surface $\mathrm{Fe}$ nanoclusters on $\mathrm{SiO}_{2}$. Applied Physics Letters 98, 053111. doi:10.1063/1.3553274

Pootawang, P., Saito, N., Takai, O., Lee, S.-Y., 2012. Synthesis and characteristics of Ag/Pt bimetallic nanocomposites by arc-discharge solution plasma processing. Nanotechnology 23, 395602. doi:10.1088/0957-4484/23/39/395602

Skandan, G, Hahn, H., Parker, J. C., 1991. Nanostructured $\mathrm{Y}_{2} \mathrm{O}_{3}$ : Synthesis and relation to microstructure and properties. Scripta metallurgica et materialia 25, 2389-2393.

Thompson, M., n.d. RUMP: Rutherford backscattering spectroscopy analysis package.

Wu Xu-Feng, X U Chun-Xiang, ZHU Guang-Ping, LING Yi-Ming, 2006. ZnO nanorods produced by the method of arc discharge. Chinese Phys. Lett. 23, 2165-2168.

Yao, W.-T., Yu, S.-H., Zhou, Y., Jiang, J., Wu, Q.-S., Zhang, L., Jiang, J., 2005. Formation of Uniform CuO Nanorods by Spontaneous Aggregation: Selective Synthesis of $\mathrm{CuO}, \mathrm{Cu}_{2} \mathrm{O}$, and $\mathrm{Cu}$ Nanoparticles by a Solid-Liquid Phase Arc Discharge Process. The Journal of Physical Chemistry B 109, 14011-14016. doi:10.1021/jp0517605

Ziegler, J. F. (Ed.), 2012. Ion Implantation Science and Technology, 2nd ed. Elsevier, Maryland, USA. 


\section{Chapter 4}

\section{Permalloy powders containing nanostructures synthesized by arc-discharge}

In this chapter, permalloy powders containing nanoparticles were synthesized by a novel arc-discharge method. The structural, magnetic and electronic properties were studied and the origin of the magnetoresistance was determined. Many studies on permalloy films have been performed indicating the origin to be anisotropic magnetoresistance (Van Gorkom et al., 2001). However, for nanoparticles of a spin polarized material and in the presence of a barrier, it is expected that there will be a tunnelling magnetoresistance along with sd scattering and it would be interesting to investigate the origins of the magnetoresistance of a permalloy pressed pellet that contains nanoparticles.

Permalloy is a well-known industrial material that has been used since the early 1920s. Since then, the material has been intensively investigated owing to its magnetic properties that include a very high permeability. Enhancement methods have focussed on additives (Elmen, 1935) and intensive processing (Chicinas et al., 2005) techniques. The initial permeability of permalloy is low and it can be as low as 500 when standard heat treatments are used (Elmen, 1935). A specialized heating, annealing and quenching method along the addition of a low concentration of other $3 \mathrm{~d}$ metals $(\mathrm{Cr}, \mathrm{Mo}, \mathrm{Mn}$ and $\mathrm{Cu})$ is used to significantly increase the permeability to values greatly exceeding 100 thousand. The permeability is highest for a permalloy concentration of $78.5 \%$. Permalloy 45 with a Ni fraction of $45 \%$ has a high saturation moment but a lower Curie temperature. There have been a few reports of iron-nickel alloy nanoparticles (Zhang et al., 1998) (Dong et al., 1999). But synthesising nanoparticles of permalloy have not been attempted before and it would be interesting to study the properties of such an alloy and investigate the origins of the magnetoresistance. 


\subsection{Arc-discharge synthesis}

Permalloy powders were synthesized using the arc-discharge system described in section 3.1.1 in Chapter 3. A permalloy rod was the anode and the cathode was Fe that sat on a water-cooled graphite disc. Rods of permalloy 45 and permalloy 78 were used. Permalloy-45 (P45) contained 45\% Ni and the remainder was predominately Fe with low concentrations of Mo, C and Mn. Permalloy-78 (P78) contained $78 \% \mathrm{Ni}$ and the remainder was predominately $\mathrm{Fe}$ with low concentrations of Mo, C and Mn. The anode and cathode were located inside a sealed chamber. The arc-discharge was carried out using a current of $74 \mathrm{~A}$ and maintaining a gap of $\sim 5 \mathrm{~mm}$ between the electrodes. The syntheses were performed in nitrogen or argon at a pressure of $\sim 400$ Torr. Two different atmospheres were used to see if the atmosphere affects the resultant composition. The powder was collected inside the base of the metal shield after arc-discharge. Some of the powder from the P78 sample made in $\mathrm{N}_{2}$ was cold pressed into a $3 \mathrm{~mm}$ diameter pellet at a pressure of $14 \mathrm{MPa}$ for magnetoresistance measurements.

The structural properties were studied by $\mathrm{XRD}$ using $\mathrm{Cu}-\mathrm{K}_{\alpha}$ radiation. SEM measurements were performed on the as-made powders that were carbon coated. High resolution TEM or HRTEM were performed on the fine powders ground in isopropanol that were dispersed on a TEM grid. Magnetic measurements were made using a MPMS from Quantum Design on the powders packed compactly in a capsule powder holder. Magnetoresistance measurements were made on a pressed pellet using the four-terminal method in a Quantum Design PPMS and with the current parallel or perpendicular to the applied magnetic field. The four-terminal magnetoresistance measurements were made by first evaporating silver electrodes and then using silver epoxy bonded contact wires. 


\subsection{Structural, magnetic and magnetoresistance measurements}

\subsubsection{X-ray diffraction}

XRD data from the P45 and P78 powders synthesized by arc-discharge in nitrogen or argon that were pressed on to a powder holder are shown in Figure 4.1. The XRD peak positions are consistent with face centred cubic (FCC) $\mathrm{Ni}_{1}$ ${ }_{x} \mathrm{Fe}_{x}$ where the space group is Fm3m (JCPDs No. 65-3244). There is no evidence for body centred cubic Fe (space group Im3m) in any of the powders. There is also no evidence for other phases in the P78 powder made in $\mathrm{N}_{2}$. The P78 powder made in Ar contains some impurity phases. Impurities are clearly seen in the P45 powders and the $\mathrm{P} 45$ powder made in $\mathrm{N}_{2}$ contains split peaks that indicate 2 permalloy phases. The impurity peaks are due to phases containing Mo, Mn, Fe, $\mathrm{Ni}$ and their oxides. The full-width half maximum of each powder is in the range of $0.2^{\circ}-0.3^{\circ}$, which is quite large and it is apparent that the particles are affected by strain. Hence, there is a large proportion of large particles present that are affected by strain. The peak at $41.9^{\circ}$ for the $\mathrm{P} 45$ made in $\mathrm{N}_{2}$ is possibly from the maghemite (marked *). The peak at 42 for the P45 made in Ar is also from maghemite (marked *). There is also a small peak in the P78 Ar, but no indication in the P78 $\mathrm{N}_{2}$. In P78 Ar, there is an indication of the Fe or Ni peak at $52^{\circ}$ and of $\mathrm{Ni}$ at $62^{\circ}$. These peaks are present in $\mathrm{P} 45 \mathrm{Ar}$ and $\mathrm{N}_{2}$ as well.

The lattice parameter was obtained for each of the powders from the XRD data and the results are listed in Table 4.1. The P78 powders have a lattice parameters, $a$, that are intermediate between that expected for $\mathrm{Ni}(3.5236 \AA$ ) and Fe $(3.6468 \AA)$. It is also close to that expected for permalloy for a similar concentration where $a=3.5556 \AA$ (Lutts and Gielen, 1970). Thus, the lattice parameters are consistent with the powders containing permalloy. 

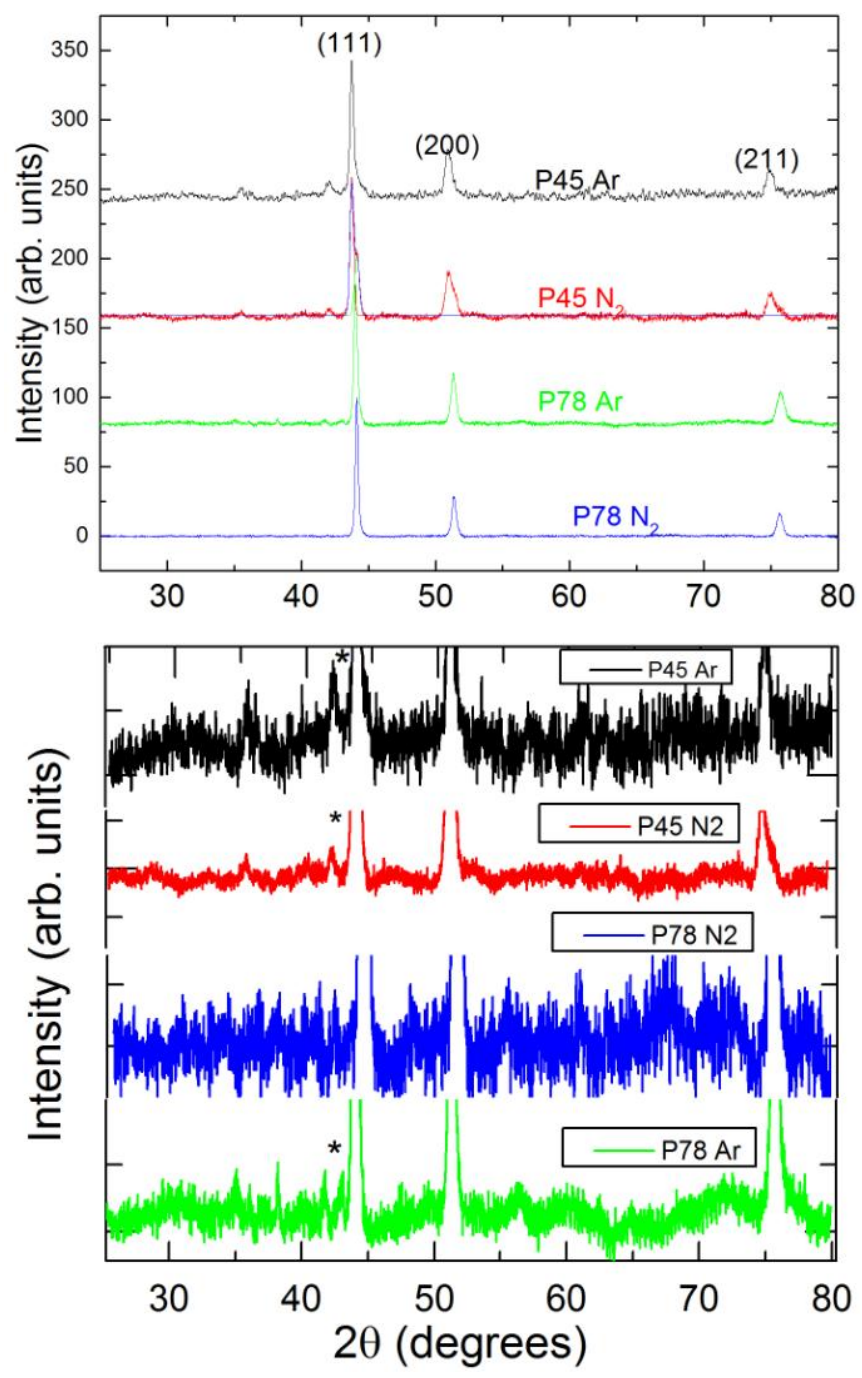

Figure 4.1. Upper figure: XRD data from P45 and P78 made in Ar or $\mathrm{N}_{2}$. The data are offset for clarity and normalized to the same (111) peak intensity. The peak from $\mathrm{P} 45 \mathrm{~N}_{2}$ is fit to two Gaussians with centres at $43.7^{\circ}$ and $44.1^{\circ}$. Lower figure: Lower intensity peaks of the spectra. The asterisk denotes the magnetite or maghemite.

The lattice parameter for P45 made in Ar is higher and based on a simple linear interpolation of the $\mathrm{Ni}$ and Fe lattice parameter, it would be expected that $a$ 
is $3.58 \AA$, which is close to the observed value. Therefore, P45 made in Ar contains a significant fraction of permalloy with a Ni content close to that of the starting material. The $\mathrm{P} 45$ powder made in $\mathrm{N}_{2}$ has split FCC peaks that indicate 2 phases where the lattice parameters are close to that expected for P45 and P78. The peak from $\mathrm{P} 45 \mathrm{~N}_{2}$ is fit to two Gaussians with centres at $43.7^{\circ}$ and $44.1^{\circ}$.

\begin{tabular}{|l|l|l|}
\hline Sample code & Gas & Lattice constant $(\AA)$ \\
\hline P45 & $\mathrm{N}_{2}$ & 3.582 and 3.55 \\
\hline P45 & $\mathrm{Ar}$ & 3.583 \\
\hline P78 & $\mathrm{Ar}$ & 3.559 \\
\hline P78 & $\mathrm{N}_{2}$ & 3.553 \\
\hline Table 4.1: Lattice parameters of the P45 and P78 samples made in nitrogen and \\
argon.
\end{tabular}

\subsubsection{Scanning electron microscopy and tunnelling electron microscopy}

SEM measurements show that all of the powders have a range of particle sizes. This is evident in Figure 4.2 (upper figure) that shows a typical SEM image of the P78 powder made in $\mathrm{N}_{2}$, where it can be seen that the particles sizes extend up to $\sim 20 \mu \mathrm{m}$. There are also some nanoparticles in the powders as can be seen in the HRTEM image in Figure 4.2 (lower left figure) of P78 made in Ar. The particle size distribution for this powder was obtained from a HRTEM image and it is shown in the lower right of Figure 4.2. The particle size distribution is typical of that seen for all of the powders where there are some small nanoparticles but the particle size distribution extends beyond $50 \mathrm{~nm}$, which is the upper limit of nanoparticle size that could be observed by HRTEM. $50 \mathrm{~nm}$ is conveniently also the critical radius for observing superparamagnetism at $300 \mathrm{~K}$. This can be shown 
from equation 2.12, $T_{\mathrm{B}}=\left[K_{\mathrm{eff}}\left(4 \pi r^{3} / 3\right)\right] /\left[25 k_{\mathrm{B}}\right]$ and taking $T_{\mathrm{B}}=300 \mathrm{~K}$ and using the measured magnetocrystalline anisotropy energy of $200 \mathrm{Jm}^{-3}$ for quenched P78 (Cullity and Graham, 2011). Thus, there are some crystalline nanoparticles with radii less than the critical superparamagnetic radii $(50 \mathrm{~nm})$ although there is a wide particle size distribution that extends up to $\sim 20 \mu \mathrm{m}$.

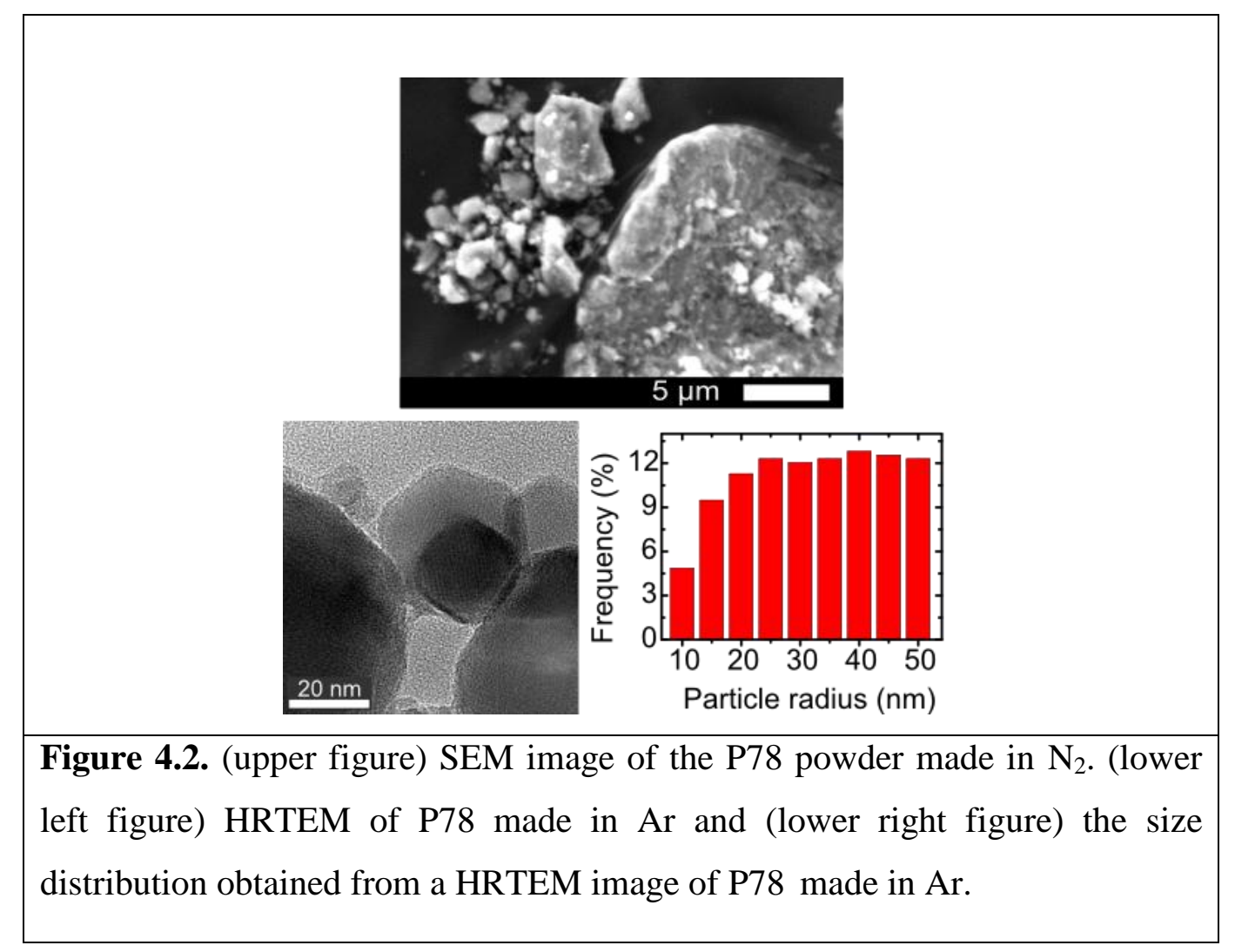

Figure 4.3 (a) shows a TEM image of an aggregate of nanoparticles. The larger particles are 60-70 $\mathrm{nm}$ and the smaller particles are below $15 \mathrm{~nm}$ wide. The particles are clustered and tend to aggregate. 
(a)

\section{$100 \mathrm{~nm}$}

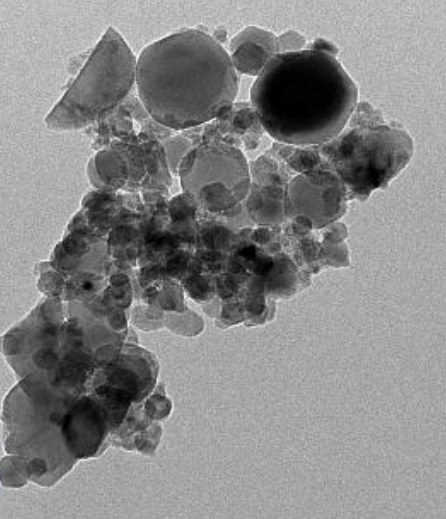

\section{(b)}

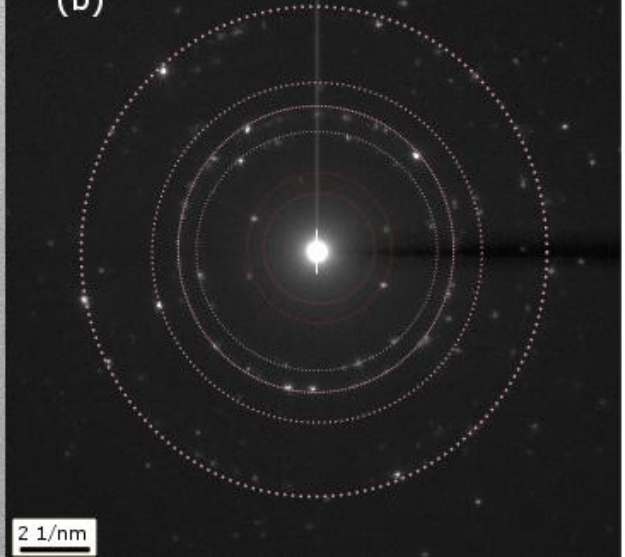

Figure 4.3 (a) TEM image of an aggregated cluster of permalloy 78 nanoparticles that were synthesised in Argon. (b) SAED pattern of powders of permalloy 78 made in Ar showing the presence of some magnetite (solid rings) and a permalloy phase (dotted rings).

Figure 4.3 (b) shows the SAED pattern of the particles of permalloy 78 powder made in Ar that has been dispersed in a TEM grid. The rings are diffuse and there are some spots. Each spot represents a lattice plane of the crystal. However, in the particles are a number of randomly oriented grains leading to a number of planes with the same lattice spacing that forms a circle. The values of $d$ spacings obtained from the SAED are given in Table 4.2. The inner rings solid rings correspond to the magnetite (220) and (311) peaks. The corresponding values of $\mathrm{d}$ spacings of magnetite are also shown in Table 4.2. This could also correspond to the maghemite phase. The outer dotted rings made by relatively brighter less diffuse spots correspond to the permalloy (111), (200) and (211) planes, as shown in Table 4.2. 


\begin{tabular}{|l|l|l|l|l|}
\hline $\begin{array}{l}\text { d spacings } \\
(\mathbf{A})\end{array}$ & $\begin{array}{l}\text { Correspondi } \\
\mathbf{n g} \mathbf{F e}_{3} \mathbf{N i} \mathbf{d} \\
\text { spacings }(\mathbf{A})\end{array}$ & Miller indices & $\begin{array}{l}\text { Corresponding } \\
\text { magnetite } \mathbf{d} \\
\text { spacings }(\AA)\end{array}$ & Miller indices \\
\hline 2.972 & & & 2.967 & 220 \\
\hline 2.544 & & & 2.532 & 311 \\
\hline 2.039 & 2.047 & 111 & & \\
\hline 1.623 & 1.7725 & 200 & & \\
\hline 1.483 & 1.253 & 220 & & \\
\hline Table 4.2 Summary of the d spacing values obtained from Figure 4.3 and the d \\
spacings of the corresponding phases and Miller indices.
\end{tabular}

\subsubsection{Magnetization measurements}

\subsubsection{Field-dependent magnetization measurements}

The magnetization data from the P78 powders and the P45 powders made in $\mathrm{N}_{2}$ and Ar are plotted in Figure 4.4 against the applied magnetic field at $5 \mathrm{~K}$ and $300 \mathrm{~K}$. The saturation magnetization of each of the powders is only slightly lower at $300 \mathrm{~K}$ compared to the $5 \mathrm{~K}$, which indicates that the Curie temperature is far above room temperature. The resulting saturation moments and coercive fields from all of the powders are listed in Table 4.3. It can be seen that $m_{\mathrm{s}} / \mu_{\mathrm{B}}$ is similar for P78 made in Ar or $\mathrm{N}_{2}$ and consistent with permalloy with similar $\mathrm{Ni}$ concentrations. P45 made in Ar has a larger $m_{\mathrm{s}} / \mu_{\mathrm{B}}$, which is expected for a larger Fe fraction. However, $m_{\mathrm{s}} / \mu_{\mathrm{B}}$ is lower for P45 made in $\mathrm{N}_{2}$. This is due to the 2 permalloy fractions that are close to P45 and P78. The values for the P45 powders are slightly less than that expected for a similar concentration of permalloy $\left(m_{\mathrm{s}} / \mu_{\mathrm{B}}\right.$ $\sim 1.8$ ( $\mathrm{Li}$ et al., 1997)). The saturation moment per formula unit for P78 made in $\mathrm{N}_{2}$ is $m_{\mathrm{S}} / \mu_{\mathrm{B}}$, is 0.74 , which is in-between that expected for $\mathrm{Fe}\left(m_{\mathrm{s}} / \mu_{\mathrm{B}}=2.2\right.$ (Li et al., 1997)) and $\mathrm{Ni}\left(m_{\mathrm{s}} / \mu_{\mathrm{B}}=0.62\right.$ (Li et al., 1997)). It is also less than that expected for bulk permalloy with a similar Ni concentration $\left(m_{\mathrm{S}} / \mu_{\mathrm{B}}=1.08\right.$ (Swartzendruber et al., 1991)). 

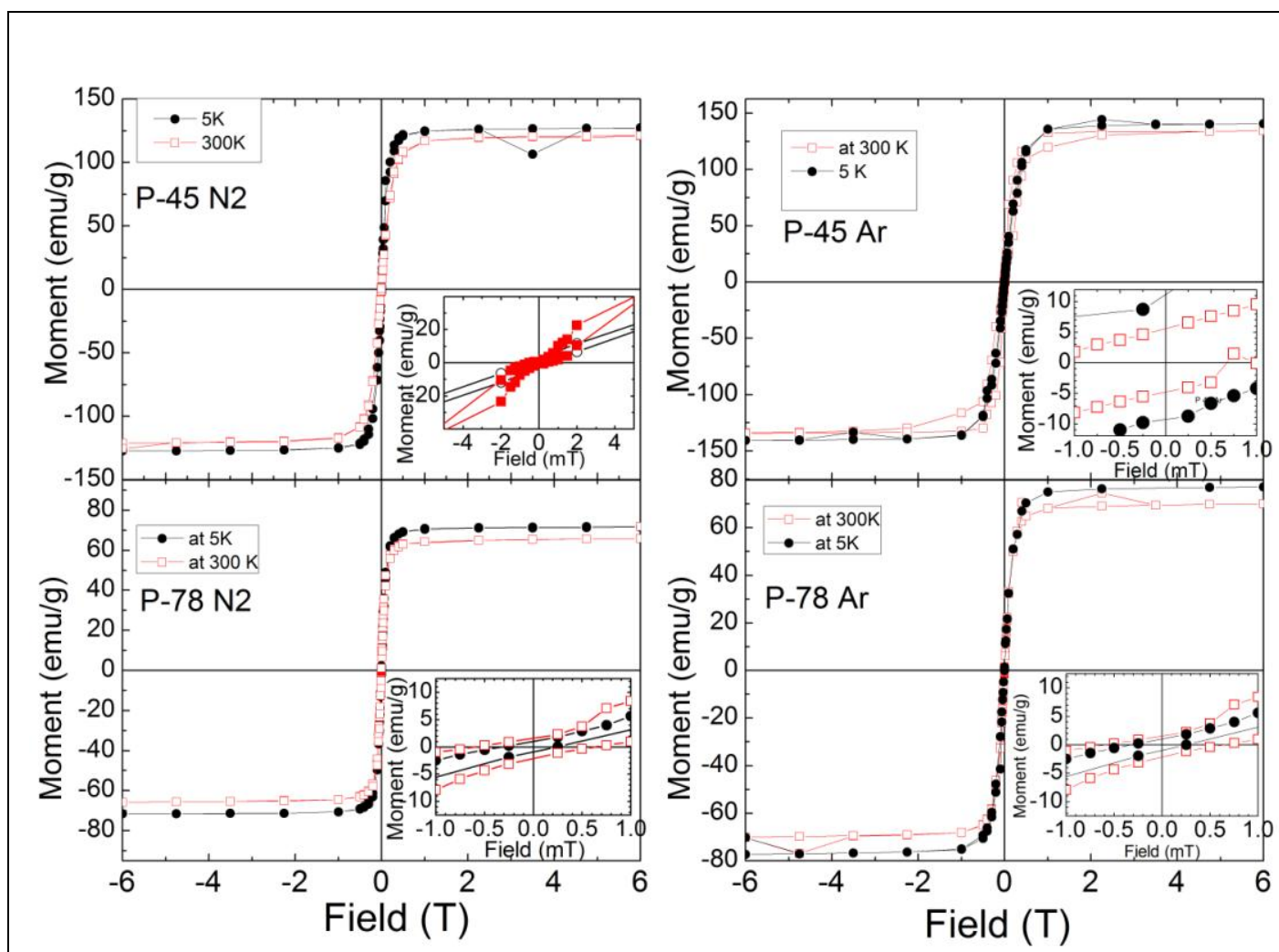

Figure 4.4. The magnetization from $\mathrm{P} 45$ made in $\mathrm{N}_{2}$ and $\mathrm{Ar}$ at $5 \mathrm{~K}$ (closed circles) and $300 \mathrm{~K}$ (open squares) and that of P78 made in $\mathrm{Ar}$ and $\mathrm{N}_{2}$ at $5 \mathrm{~K}$ (closed circles) and $300 \mathrm{~K}$ (open squares) made in $\mathrm{N}_{2}$. The inset shows the same data for small magnetic fields.

It is apparent in the insets to Figure 4.4 that there is some hysteresis at $5 \mathrm{~K}$ and $300 \mathrm{~K}$. This is expected because most of the particles are greater than $50 \mathrm{~nm}$ and hence the hysteresis arises from these non-superparamagnetic particles. The coercive field is low at $300 \mathrm{~K}$ and it is $0.3 \mathrm{mT}$. This is consistent with permalloy that has Ni concentrations comparable to P78 because of domain wall pinning.

The saturation field for P45 and the P78 is high at it is $~ 0.6 \mathrm{~T}$. This is far higher than that expected for permalloy that has undergone the specialized processing method. It is even higher than that found in permalloy processed using standard methods. It is likely to be high owing to significant $\mathrm{Ni}$ and $\mathrm{Fe}$ site 
disorder as well as crystal defects and impurities that result in domain wall pinning. It is also likely that it is large owing to demagnetization effects.

\begin{tabular}{|c|c|c|c|}
\hline Sample code & Gas & $m_{\mathrm{s}} / \mu_{\mathrm{B}}($ at $5 \mathrm{~K})$ & $B_{\mathrm{c}}(\mathrm{mT})$ \\
\hline P45 & $\mathrm{N}_{2}$ & 1.30 & 0.7 \\
\hline P45 & Ar & 1.48 & 0.5 \\
\hline P78 & Ar & 0.78 & 1.6 \\
\hline P78 & $\mathrm{N}_{2}$ & 0.74 & 0.3 \\
\hline
\end{tabular}

Table 4.3. Table of the saturation moment per formula unit at $5 \mathrm{~K}$, and the coercive fields at $300 \mathrm{~K}$ for P45 and P78 made in different gases.

\subsubsection{Temperature-dependent magnetization measurements}

Zero-field cooled (ZFC) and field-cooled (FC) measurements were performed on the powder sample at $10 \mathrm{mT}$ in Figure 4.5. There is still irreversibility up to the highest temperature that indicates that any blocking temperature is above $300 \mathrm{~K}$. This is indicative of particle sizes greater than $50 \mathrm{~nm}$, consistent with the particles seen in the electron microscopy images. A transition at $\sim 110 \mathrm{~K}$ can be seen in the ZFC and FC curves. This is possibly from the presence of magnetite in the powder that has a charge ordering Verwey transition at $\sim 120 \mathrm{~K}$. The occurrence of Verwey transition in the magnetization data is discussed in more detail in the following chapter that deals with magnetite (Chapter 5). 


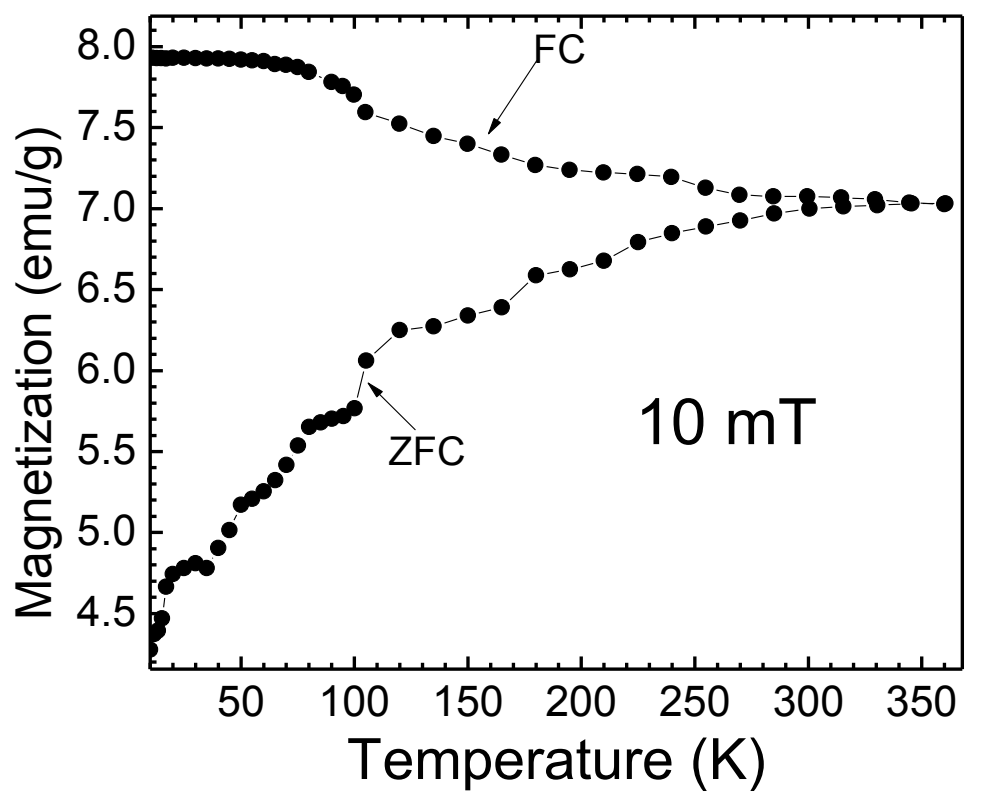

Figure 4.5. ZFC-FC curves of the magnetization from $\mathrm{P} 78$ made in $\mathrm{Ar}$ in the range from $5 \mathrm{~K}$ to $360 \mathrm{~K}$, in the presence of a magnetic field of $10 \mathrm{mT}$.

High-field temperature-dependent measurements were performed on the permalloy powders from $5 \mathrm{~K}$ to $360 \mathrm{~K}$. Figure 4.6 shows a plot of the data from P78 made in Ar. The saturation magnetization decreases with increasing temperature due to spin-waves. The data were fitted to Eq. 2.11, $M_{\mathrm{s}}=M_{0}\left[1-B T^{\beta}\right]$, where $M_{0}$ is the magnetization saturation at $0 \mathrm{~K}$. It can be seen in Figure 4.6 that the fit is not good at low temperatures where there is an additional decrease in the saturation magnetization to $\sim 25 \mathrm{~K}$. This initial decrease may come from parts of the powder that are spin-disordered. This is discussed in chapter 6 where an initial decrease is seen in $\mathrm{Ni}_{1-\mathrm{x}} \mathrm{Fe}_{\mathrm{x}}$ implanted nanoparticles and attributed to a spin-disordered region in the nanoparticle shells and a range of spin-freezing temperatures. It is likely that this also occurs in the P78 made by arc-discharge as well as spin disorder in larger particles. The fit gives $\beta=1.96$, a Bloch constant of $1.03 \mathrm{E}-6 \mathrm{~K}^{-1.96}$ and a saturation magnetization of $64 \mathrm{emu} / \mathrm{g}$ at 
$0 \mathrm{~K}$. The Bloch constant is higher than the values reported on permalloy nanoparticles (Zhang et al., 1998). The higher Bloch exponent compared to that expected $\left(\beta_{\text {bulk }}=1.5\right)$ for a ferromagnet may be due to large particles that are strained.

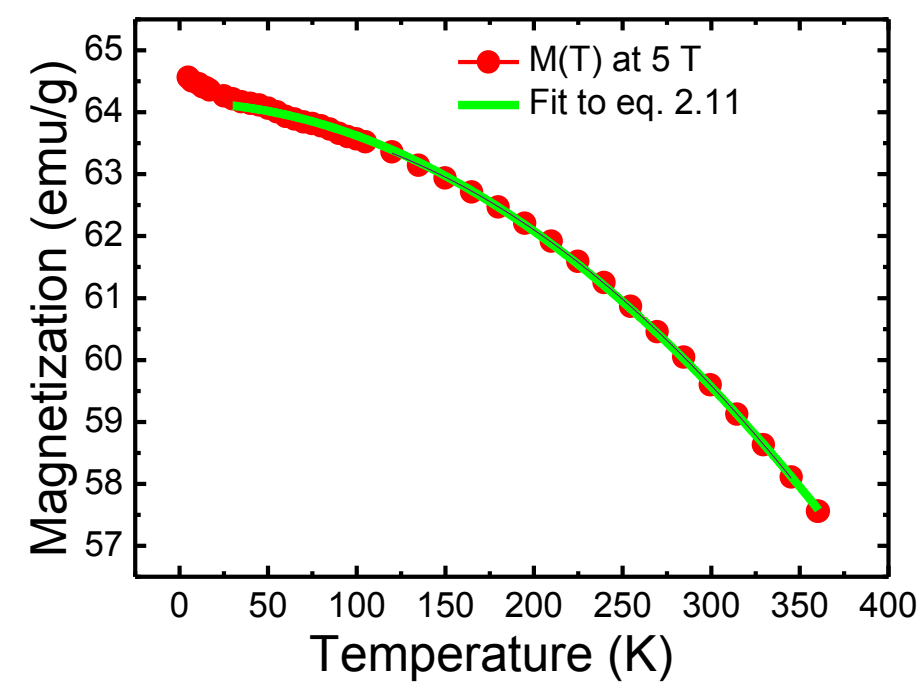

Figure 4.6. Temperature-dependent curves of the magnetization from P78 made in Ar at 5 T. Also shown is a fit to equation 2.11.

The relative permeability of the P78 powder made in Ar was calculated to be a maximum of 10 at $25 \mathrm{mT}$ and this is plotted in Figure 4.7. It is approximately the same for all of the powders. The permeability is not as high as expected, which may be due to demagnetization effects discussed in Chapter 3 from the particles in the powder. 


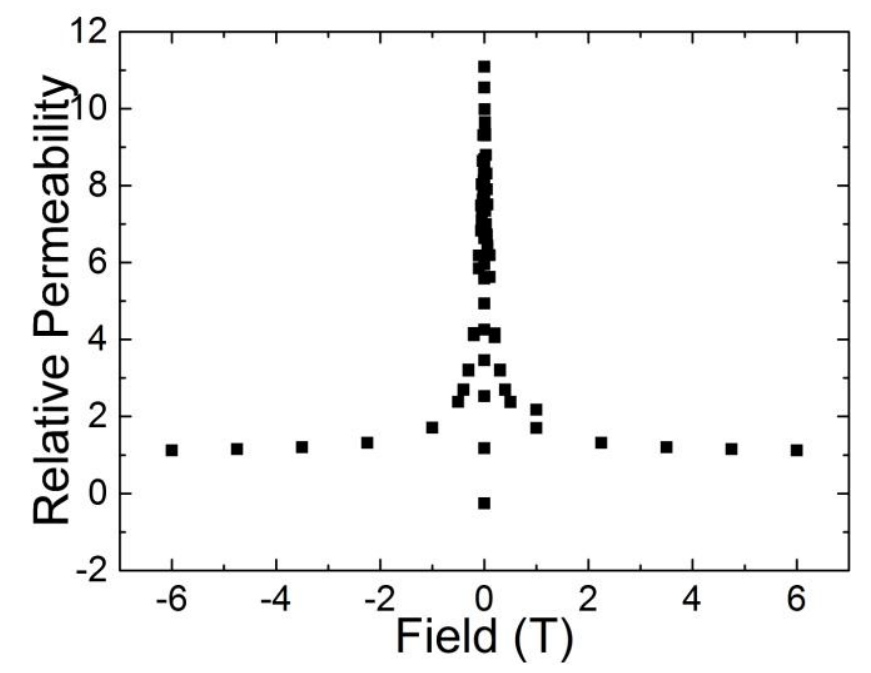

Figure 4.7. Permeability of P78 powder made in Ar at room temperature.

\subsubsection{Magnetoresistance measurements}

Electrical resistance measurements were performed on the pressed pellet of P78 made in N2 and the results are plotted in Figure 4.8. Temperature is plotted against the normalized resistance, which is the resistance normalized to the highest resistance value. Measurements were done in the absence of field in order to determine whether there was any deviation from the expected linear behavior of metals (Milchberg et al., 1988) that could indicate intergranular tunneling or variable range hopping (Sheng et al., 1973). The resistance showed a linear dependence that is a result of the phonon scattering. 


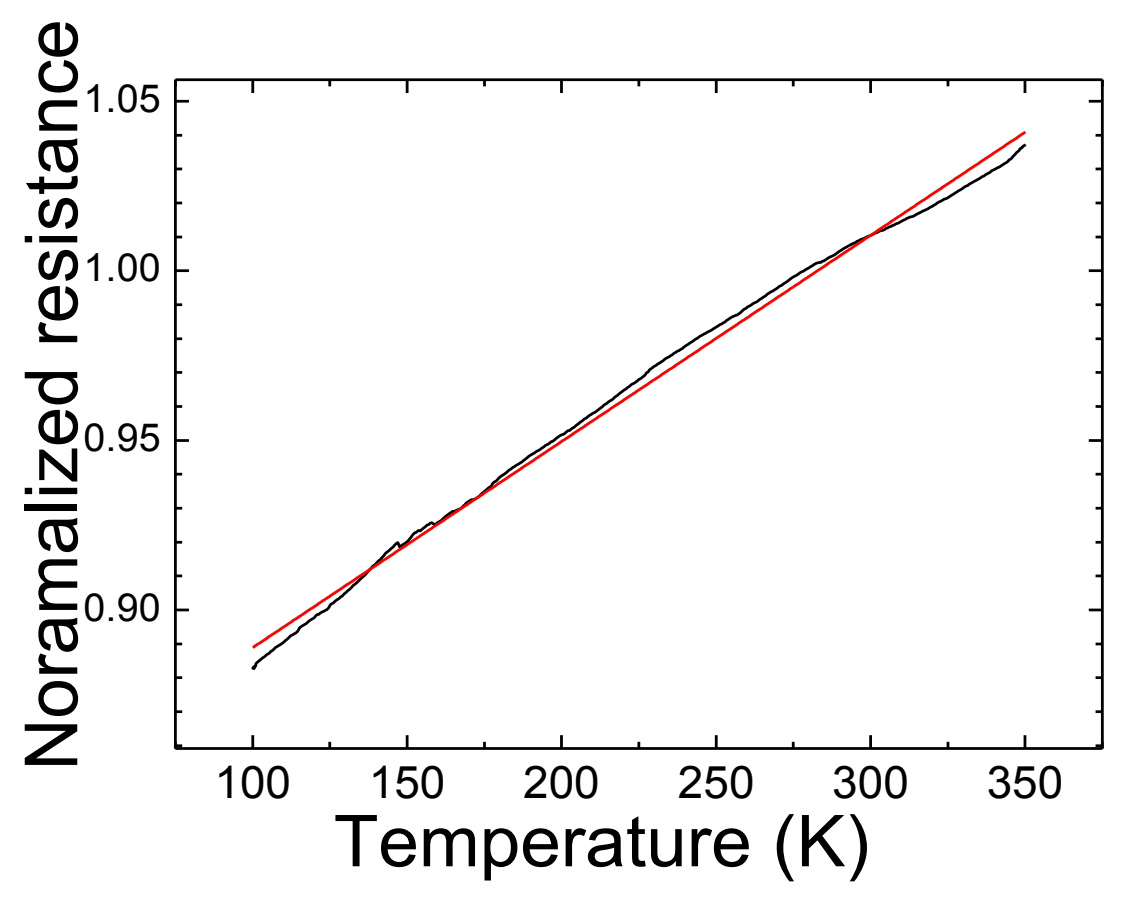

Figure 4.8: Normalized electrical resistance for the P78 (N2) pressed pellet.

Magnetoresistance measurements were performed on a pressed pellet of P78 made in $\mathrm{N}_{2}$. The magnetoresistance is defined as $\mathrm{MR}(B)=(R(B)-R(0)) / R(0)$ where $R(B)$ is the resistance for an applied magnetic flux density, $B$, and $R(0)$ is the resistance when $B=0$. This powder was chosen because there was no evidence of impurity phases in the XRD data. The magnetoresistance at $5 \mathrm{~K}, 100 \mathrm{~K}$ and $300 \mathrm{~K}$ are shown in Figure 4.9. At low temperatures and at low fields, the magnitude of the magnetoresistance increases with increasing field and then there is an upturn where the magnetoresistance decreases with increasing field. This is similar for the magnetoresistance at $100 \mathrm{~K}$ where the magnetoresistance decreases with increasing field till $1.5 \mathrm{~T}$ and then is nearly constant, varying only from $0.22 \%$ to $0.25 \%$. The magnitude of the magnetoresistance is only $0.21 \%$ for $100 \mathrm{~K}$ and there is a less of a decrease of the magnetoresistance at higher fields. The magnetoresistance at $300 \mathrm{~K}$ is even less so and there is only a continual increase with field. The magnetoresistance at $300 \mathrm{~K}$ is $0.59 \%$ at $8 \mathrm{~T}$. 


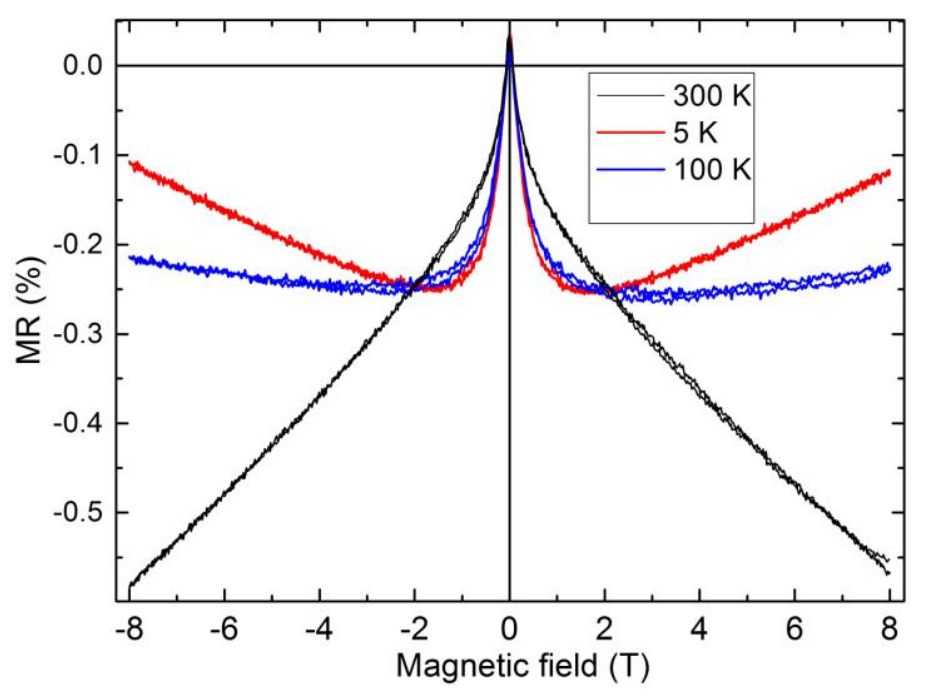

Figure 4.9: The magnetoresistance of the pressed pellet of $\mathrm{P} 78$ made in $\mathrm{N}_{2}$ at the temperatures of $5 \mathrm{~K}, 100 \mathrm{~K}$ and $300 \mathrm{~K}$.

Magnetoresistance measurements in the low field range $(-20 \mathrm{mT}$ to $20 \mathrm{mT}$ ) showed a larger coercivity than that seen from the magnetization (see Figure 4.10). The sweep rate used was $0.1 \mathrm{mT} / \mathrm{s}$. There is a peak in the magnetoresistance at $\sim 0.05 \mathrm{~T}$, which is much higher than that seen in the field dependant magnetization. The magnetizations of the grains point randomly at low fields and results in a higher resistance state compared to that at higher fields. This is typical of spin polarized materials with grain boundary regions (Serrate et al., 2005b). Spin dependant scattering can cause a high resistivity as electrons travel across grains. There is a low resistivity in the presence of a lower field when the domains are aligned (Gupta et al., 1996). Intergrain tunnelling magnetoresistance has been observed in polarized materials with grain boundaries (Serrate et al., 2005b). The presence of a 'butterfly' loop at low fields has also been observed in systems with spin orbit interaction resulting in a positive magnetoresistance at low fields, or weak localization (Gunnarsson, 2012; Milchberg et al., 1988). 


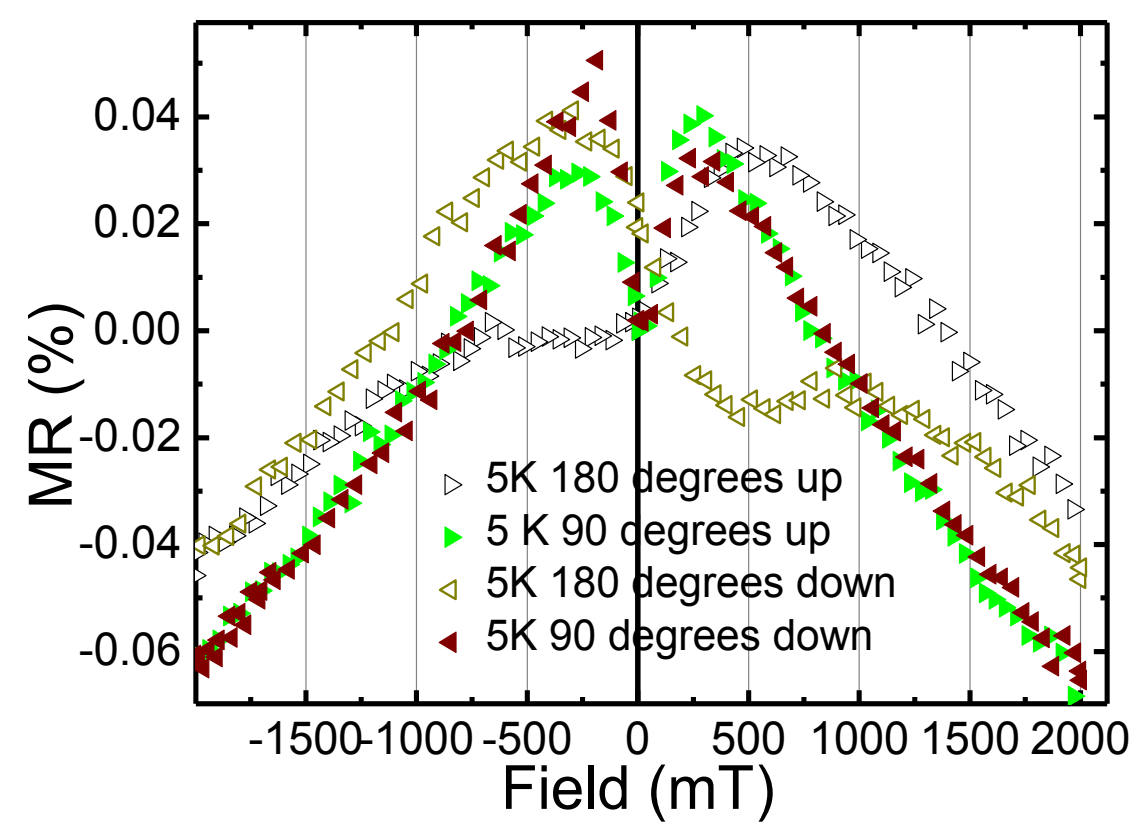

Figure 4.10. (color online) The magnetoresistance from P78 made in $\mathrm{N}_{2}$ at $5 \mathrm{~K}$ as a function of the field perpendicular and parallel to the field.

It is possible that the decreasing magnetoresistance at moderate $\mathrm{B}$ $(20 \mathrm{mT}<\mathrm{B}<1.5 \mathrm{~T})$ and at $5 \mathrm{~K}$ is due to spin-dependent tunnelling between the permalloy particles because permalloy has a degree of electronic spin polarization. Spin-dependent tunnelling has previously been reported in electronically spin polarized thin films (Žutić et al., 2004) (Prinz, 1998), polycrystalline samples (Serrate et al., 2007) (Kobayashi et al., 1998) (Hemery et al., 2007), and nanogranular materials (Liu et al., 2003) (Serrate et al., 2005a) (Yue et al., 2011) (Inoue and Maekawa, 1996). It occurs at the interface between two magnetic domains that are not exchanged coupled (e.g. when there is an insulating barrier) (Žutić et al., 2004) (Liu et al., 2003) (Serrate et al., 2005a) (Inoue and Maekawa, 1996) (Prinz, 1998) (Kobayashi et al., 1998) (Serrate et al., 2007) (Hemery et al., 2007). The resulting magnetoresistance for granular materials can be written as 
(Inoue and Maekawa, 1996), from equation 2.16, $\mathrm{MR}(\mathrm{B})=-\frac{f^{2} m(B)^{2}}{1+f^{2} m(B)^{2}}$, where $m(B)$ is the reduced magnetization and $f$ is the carrier spin polarized fraction. $f$ is 0.35 for $\mathrm{P} 78$ at low temperatures and hence from equation 2.16 the magnetoresistance should be $\sim-11 \%$ when the magnetization has saturated, which is above $\sim 0.6 \mathrm{~T}$ as can be seen in Figure 4.9. However, it is apparent in Figure 4.9 that the magnetoresistance at $5 \mathrm{~K}$ only reaches $\sim-0.26 \%$.

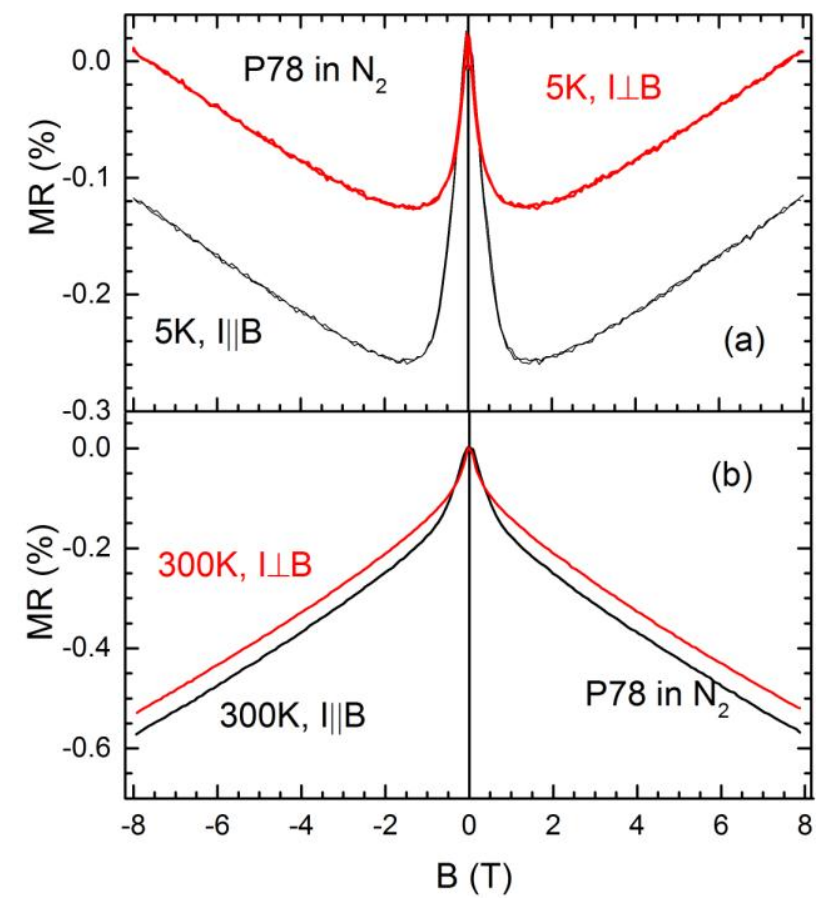

Figure 4.11: (a) The magnetoresistance from P78 made in $\mathrm{N}_{2}$ at $5 \mathrm{~K}$ for the current parallel or perpendicular to $B$. (b) The magnetoresistance from P78 made in $\mathrm{N}_{2}$ at $300 \mathrm{~K}$ where the current was perpendicular or parallel to $B$.

Magnetoresistance values smaller than those expected from equation 2.16 have also observed in other granular (Serrate et al., 2007) (Hemery et al., 2007) and nanoparticle (Liu et al., 2003; Serrate et al., 2005a; Yue et al., 2011) 
materials. It can be caused by disorder near and at the interface that leads to spinflip scattering and degrades the spin polarized current (Serrate et al., 2007).

The results $5 \mathrm{~K}$ and $300 \mathrm{~K}$ for the current parallel or perpendicular to $B$ are shown in Figure 4.11. The increasing magnetoresistance at high $B(>1.5 \mathrm{~T})$ and $5 \mathrm{~K}$ includes a large contribution from the OMR. This is a Lorentz force effect that is positive and can be proportional to $B^{2}$ (Van Gorkom et al., 2001) (Jan, 1957) (Falicov and Sievert, 1965). However, the exact magnetic field dependence depends on the band structure (Van Gorkom et al., 2001). In this case the resistivity for the current perpendicular to $B$ is larger than the resistivity for the current parallel to $B$. This is in fact observed at $8 \mathrm{~T}$ and $5 \mathrm{~K}$ as can be seen in Figure 4.11.

Further evidence for an OMR contribution to the high field magnetoresistance can be seen in Figure 4.12 where the resistance is plotted against the angle between the current and the applied magnetic field. We find that the resistance can be fitted to the expected OMR MR angle dependence that can be written as,

$$
R(\vartheta)=R_{\mathrm{para}}+\left[R_{\mathrm{perp}}-R_{\mathrm{para}}\right] \times[\sin (\vartheta)]^{2}
$$

where $\vartheta$ is the angle between $B$ and the current, $R_{\text {perp }}$ is the resistivity for $\vartheta=90^{\circ}$, and $R_{\text {para }}$ is the resistivity for $\vartheta=0^{\circ}$. For anisotropic magnetoresistance due to s-d scattering the $\sin (\vartheta)^{2}$ angle dependence is replaced by a $\cos (\vartheta)^{2}$ angle dependence that is clearly not evident and hence any anisotropic magnetoresistance contribution is small.

The magnetoresistance at $300 \mathrm{~K}$ (Figure $4.11 \mathrm{~b}$ ) systematically decreases with increasing $B$. The absence of an upturn in the magnetoresistance at high fields and the weak magnetoresistance anisotropy when compared with the $5 \mathrm{~K}$ data may be because the OMR contribution is small. This is known to occur in metals because the OMR also depends on the mean free path and hence the OMR 
can be larger at lower temperatures (Van Gorkom et al., 2001). Thus, the magnetoresistance at $300 \mathrm{~K}$ is dominated by spin-dependent tunnelling. The magnetoresistance decreases with increasing $B$ even above the saturation $B$ of $\sim 0.6 \mathrm{~T}$, which is not expected from equation 4.1. However, it can occur when there is disorder near the interface regions and hence the reduced magnetization in this disordered region should be used in equation 4.1 (Serrate et al., 2007) (Hemery et al., 2007). In one magnetoresistance model, the reduced magnetization near the surface of each particle at high $B$ approaches that of a non-saturating spin-glass, which results in a magnetoresistance that continually decreases with increasing $B$ (Serrate et al., 2007).

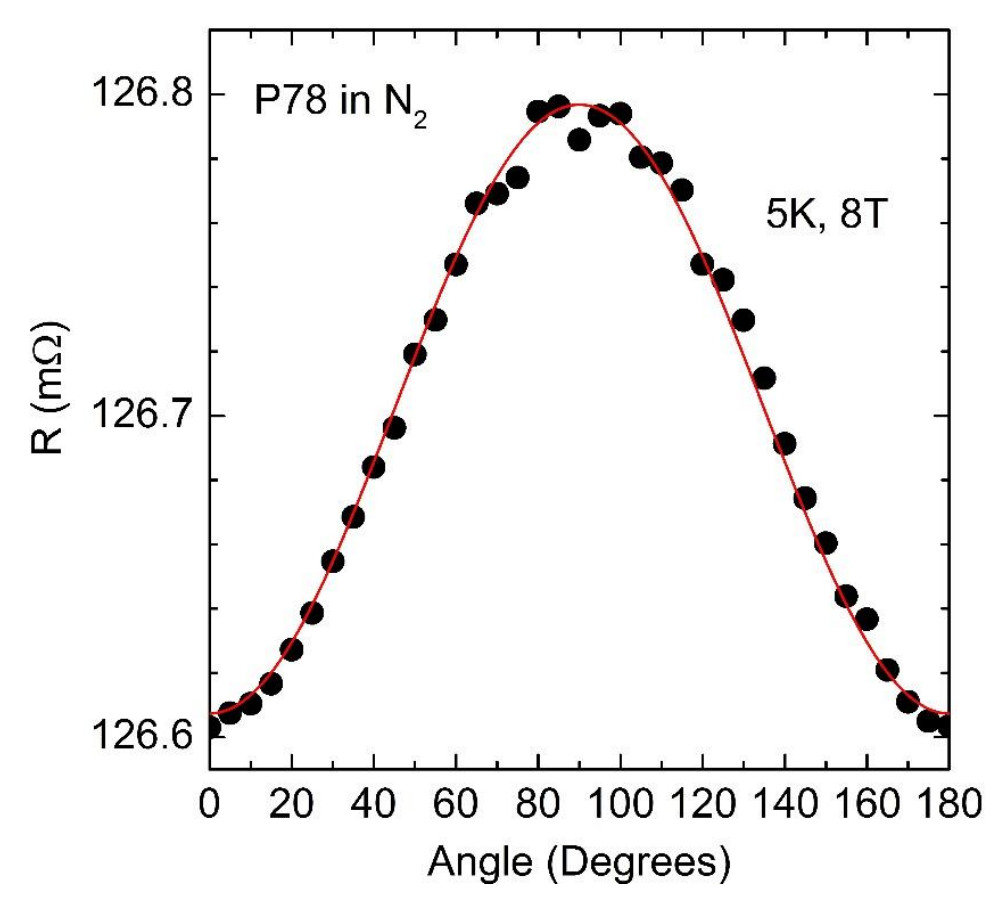

Figure 4.12. (color online) The resistance from $P 78$ made in $\mathrm{N}_{2}$ at $5 \mathrm{~K}$ as a function of the angle between the current and applied magnetic field at $8 \mathrm{~T}$. The solid curve is a fit to the data using Equation 4.1. 
This spin-disorder model explains the non-saturation of the magnetoresistance provided that the volume fraction of this spin-disordered region was significantly less than that from the ferromagnetically ordered core regions, otherwise the measured magnetization would continually increase to high magnetic fields, which is not observed (see Figure 4.4). The presence of a coercivity in the magnetoresistance (Figure 4.10) has also been seen in systems with intergranular tunnelling magntoresistance (Serrate et al., 2005b). In our case the SEM and magnetization data show that while there are some small nanoparticles, most of the particle sizes are beyond the superparamagnetic limit $(>50 \mathrm{~nm})$ and the particle sizes extend up to $20 \mu \mathrm{m}$. Thus, there is a spindisordered region in most of the particles that extends tens of nanometres into the particles and where the magnetoresistance is dominated by spin-transport in these regions and between the particles.

\subsubsection{Improving the design of the arc-discharge system}

Further work will focus on altering the arc-discharge design to maximize the nanoparticle volume fraction. A schematic of the particle aggregation is shown in Figure 4.13 (right), where the finer particles are seen to aggregate away from the arcing area and the larger coalesced particles are seen closer to the cathode. The present collection point is the area close to the arcing and a possible design of the arc-discharge would be to allow for a collection point that is toward the outer walls where the finer particles are found. Another modification would be rotating the rods by ninety degrees and allowing the powder to drop into a solution and use the small particles that are suspended in the solution. 


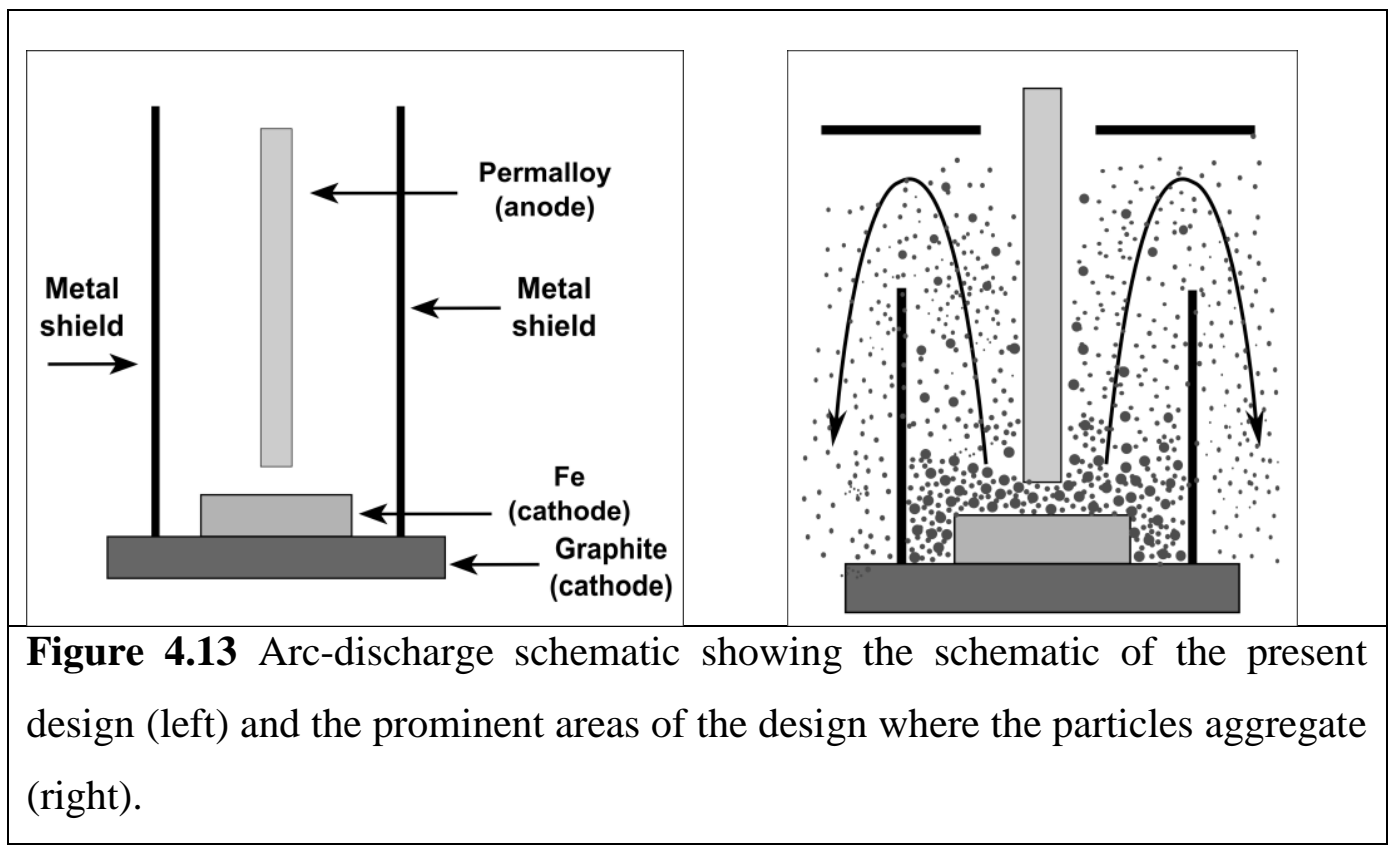




\subsection{Conclusions}

In conclusion, permalloy powders have been made by arc-discharge using permalloy rods with $78 \%$ or $45 \% \mathrm{Ni}$ concentrations and made in $\mathrm{N}_{2}$ or Ar. The resulting powders contain permalloy with some nanoparticles as well as particles up to $\sim 20 \mu \mathrm{m}$ in diameter. The highest quality powders were made using a P78 anode in $\mathrm{N}_{2}$ and the resulting powder composition was close to that expected for P78. $m_{s} / \mu_{\mathrm{B}}$ was less than that expected in the bulk and the coercive field was as low as $0.3 \mathrm{mT}$. The permeability was low, which may be due to demagnetization effects. Magnetoresistance measurements were made on a cold pressed pellet containing the $\mathrm{P} 78$ powder made in $\mathrm{N}_{2}$ and a magnetoresistance was observed that had significant contributions from the ordinary magnetoresistance and spindependent tunnelling between the particles. The low magnetoresistance is attributed to the presence of spin disorder at the boundary region.

\section{References}

Bozorth, R.M. and Ferromagnetism, D., 1951. Van Nostrand Company. New York, 849

Chicinas, I., Geoffroy, O., Isnard, O., Pop, V., 2005. Soft magnetic composite based on mechanically alloyed nanocrystalline Ni3Fe phase. J. Magn. Magn. Mater. 290-291, 1531-1534. doi:10.1016/j.jmmm.2004.11.249

Cullity, B.D., Graham, C.D., 2011. Introduction to magnetic materials. John Wiley \& Sons.

Dong, X.L., Zhang, Z.D., Zhao, X.G., Chuang, Y.C., Jin, S.R., Sun, W.M., 1999. The preparation and characterization of ultrafine $\mathrm{Fe}-\mathrm{Ni}$ particles. J. Mater. Res. 14, 398-406.

Elmen, G., 1935. Magnetic Alloys of Iron Nickel, and Cobalt. Am. Inst. Electr. Eng. Trans. Of 54, 1292-1299.

Falicov, L.M., Sievert, P.R., 1965. Theory of the galvanomagnetic effects in metals with magnetic breakdown: Semiclassical approach. Phys. Rev. 138, A88-A98. doi:10.1103/PhysRev.138.A88

Gunnarsson, R., 2012. Anisotropic spin-orbit interaction revealed by in-plane magnetoresistance in single-oriented SrRuO 3 thin films. Phys. Rev. B 85. doi:10.1103/PhysRevB.85.235409 
Gupta, A., Gong, G.Q., Xiao, G., Duncombe, P.R., Lecoeur, P., Trouilloud, P., Wang, Y.Y., Dravid, V.P., Sun, J.Z., 1996. Grain-boundary effects on the magnetoresistance properties of perovskite manganite films. Phys. Rev. B 54, R15629-R15632. doi:10.1103/PhysRevB.54.R15629

Hemery, E.K., Williams, G.V.M., Trodahl, H.J., 2007. The effect of isoelectronic substitution on the magneto-resistance of Sr2-xBaxFeMoO6. Phys. B Condens. Matter 390, 175-178. doi:10.1016/j.physb.2006.08.010

Inoue, J., Maekawa, S., 1996. Theory of tunneling magnetoresistance in granular magnetic films. Phys. Rev. B 53, R11927.

Jan, J.-P., 1957. Galvamomagnetic and Thermomagnetic Effects in Metals, Solid State Physics - Advances in Research and Applications.

Kobayashi, K.-I., Kimura, T., Sawada, H., Terakura, K., Tokura, Y., 1998. Roomtemperature magnetoresistance in an oxide material with an ordered double-perovskite structure. Nature 395, 677-680. doi:10.1038/27167

Liu, K., Zhao, L., Klavins, P., Osterloh, F.E., Hiramatsu, H., 2003. Extrinsic magnetoresistance in magnetite nanoparticles. J. Appl. Phys. 93, 79517953. doi:10.1063/1.1556133

Li, X., Chiba, A., Takahashi, S., 1997. Preparation and magnetic properties of ultrafine particles of Fe-Ni alloys. J. Magn. Magn. Mater. 170, 339-345.

Lutts, A., Gielen, P.M., 1970. . Phys Status Solidi 40, 81.

Milchberg, H.M., Freeman, R.R., Davey, S.C., More, R.M., 1988. Resistivity of a simple metal from room temperature to 106 K. Phys. Rev. Lett. 61, 2364.

Prinz, G.A., 1998. Magnetoelectronics. Science 282, 1660-1663.

Serrate, D., De Teresa, J.M., Algarabel, P.A., Fernández-Pacheco, R., Galibert, J., Ibarra, M.R., 2005a. Grain-boundary magnetoresistance up to $42 \mathrm{~T}$ in cold-pressed Fe3O4 nanopowders. J. Appl. Phys. 97. doi:10.1063/1.1868877

Serrate, D., De Teresa, J.M., Algarabel, P.A., Ibarra, M.R., Galibert, J., $2005 b$. Intergrain magnetoresistance up to $50 \mathrm{~T}$ in the half-metallic ( $\mathrm{Ba} 0.8 \mathrm{Sr} 0.2$ ) 2 FeMoO 6 double perovskite: Spin-glass behavior of the grain boundary. Phys. Rev. B 71. doi:10.1103/PhysRevB.71.104409

Serrate, D., Teresa, J.M.D., Ibarra, M.R., 2007. Double perovskites with ferromagnetism above room temperature. J. Phys. Condens. Matter 19, 023201. doi:10.1088/0953-8984/19/2/023201

Sheng, P., Abeles, B., Arie, Y., 1973. Hopping conductivity in granular metals. Phys. Rev. Lett. 31, 44.

Swartzendruber, L., Itkin, V., Alcock, C., 1991. The Fe-Ni (iron-nickel) system. J Phase Equilibria 12, 288-312.

Van Gorkom, R.P., Caro, J., Klapwijk, T.M., Radelaar, S., 2001. Temperature and angular dependence of the anisotropic magnetoresistance in epitaxial $\mathrm{Fe}$ films. Phys. Rev. B - Condens. Matter Mater. Phys. 63, 13443211344329.

Yue, F.J., Wang, S., Lin, L., Zhang, F.M., Li, C.H., Zuo, J.L., Du, Y.W., Wu, D., 2011. Large low-field magnetoresistance in $\mathrm{Fe} 3 \mathrm{O} 4 /$ molecule nanoparticles 
at room temperature. J. Phys. Appl. Phys. 44. doi:10.1088/00223727/44/2/025001

Zhang, D., Klabunde, K.J., Sorensen, C.M., Hadjipanayis, G.C., 1998.

Magnetization temperature dependence in iron nanoparticles. Phys. Rev. B 58, 14167.

Žutić, I., Fabian, J., Sarma, S.D., 2004. Spintronics: Fundamentals and applications. Rev. Mod. Phys. 76, 323. 


\section{Chapter 5}

\section{Iron oxide made by arc-discharge method and a co- precipitation method}

This chapter looks at the synthesis of powders of the highly spin polarized material magnetite and its structural, magnetic and magnetoresistance properties. Magnetite was made by a novel arc-discharge method and then using a conventional chemical co-precipitation method. The results from the two different preparation methods are compared in the summary.

\subsection{Iron oxide nanostructured particles with a spin disordered region at the interface}

In this section, the properties of magnetite prepared by a novel arcdischarge method are discussed. The permalloy powders made in chapter 4 showed a magnetoresistance of $-0.26 \%$ at $300 \mathrm{~K}$ and $8 \mathrm{~T}$. It would be interesting to investigate the magnetoresistance of magnetite powders made by a similar method. It would also be interesting to understand the contribution to the spin disorder in granular materials that leads to a decrease in the spin polarization that was observed for the permalloy. The magnetic and magnetoresistance properties are investigated and the contribution to the spin disorder is modelled.

\subsubsection{Synthesis technique}

Iron oxide powders were synthesized using the arc-discharge system at GNS Science (Prakash et al., 2014),(Fang et al., 2009). The anode was an iron rod and the cathode was a thick $\mathrm{Fe}$ disc that sat on a larger diameter water-cooled 
graphite disc. They were located in a sealed chamber where the separation between the anode was attached to a stepper motor so that it could be raised or lowered to create a $\sim 5 \mathrm{~mm}$ gap. An electrical current of $74 \mathrm{~A}$ was used to create the arc. The iron powder synthesis was performed in an oxygen atmosphere at a pressure of $\sim 53 \mathrm{kPa}$. The powder at the base of the metal shield was collected and cold pressed at $14 \mathrm{MPa}$ into $3 \mathrm{~mm}$ diameter pellets for four-terminal magnetoresistance measurements. The electrical contacts were made by first evaporating silver electrodes and then using silver epoxy bonded contact wires.

XRD was done using Co $K_{\alpha}$ radiation $(\lambda=1.78 \AA)$ to determine the phase composition of the powder. SEM was performed using a JEOL JSM 6610F after carbon coating of the samples using a Quorum $150 \mathrm{~T}$ carbon coater. The SEM was operated in the secondary electron mode in order to obtain the surface topography. HRTEM images of powders were taken using a Tecnai TF20 with an acceleration voltage of $200 \mathrm{kV}$ at the University of Melbourne. SAED was also recorded. Confocal microscope Raman spectroscopy was performed using a LabRam equipped with a cooled charge-coupled device (CCD) detector. The Raman spectra were collected using the $514.5 \mathrm{~nm}$ line from an argon ion laser. The laser spot size was $\sim 1 \mu \mathrm{m}$. Magnetic measurements were performed on the powder using a vibrating sample magnetometer in a PPMS from Quantum Design as described in Chapter 2. Magnetoresistance measurements were made using the four-terminal method in a Quantum Design PPMS and with the current parallel to the applied magnetic field.

\subsubsection{Structural analysis}

\subsubsection{X-ray diffraction}

The XRD data from the iron oxide powders are shown in Figure 5.1.1. The prominent peak positions in the powder synthesized by arc-discharge at $35.22^{\circ}$, 
$41.46^{\circ}, 50.57^{\circ}, 63.04^{\circ}, 65.35^{\circ}$ and $74.23^{\circ}$ correspond to magnetite, indicating that the structure is cubic with the space group Fd $\overline{3} \mathrm{~m}$ (JCPDS pattern no. 019-0629).

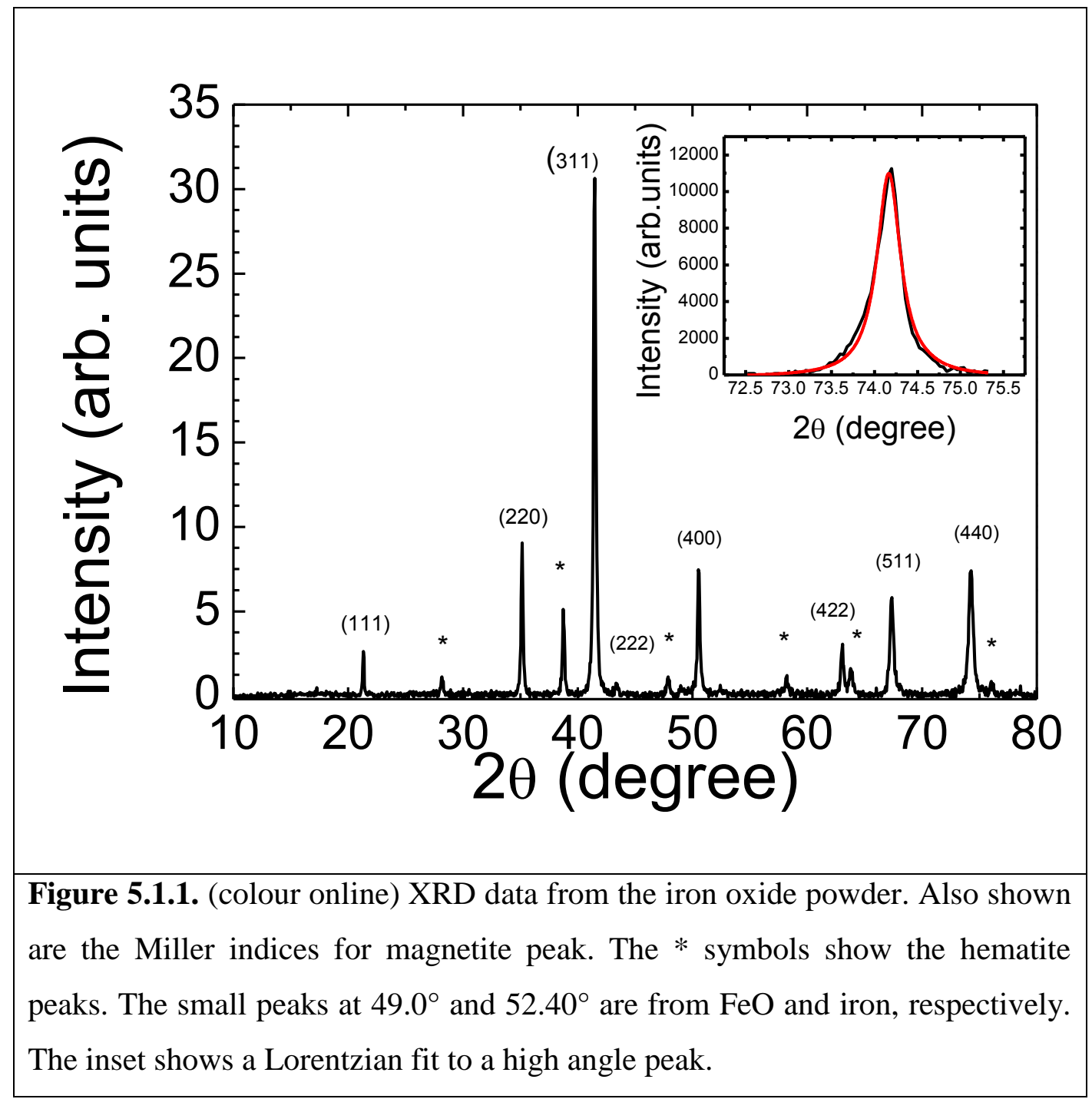

There is also an additional phase clearly indicated by the low intensity peaks (marked with $*$ ) attributed to a low concentration of hematite $\left(\alpha-\mathrm{Fe}_{2} \mathrm{O}_{3}\right)$, which has a rhombohedral crystal system phase (R-3c, JCPDS pattern no. 0330664). Hematite is a semiconductor that antiferromagnetically orders at $263 \mathrm{~K}$. There is an indication of the presence of body centred cubic $\mathrm{Fe}$ (space group 
$\mathrm{Im} 3 \mathrm{~m})$ at $52.4^{\circ}$. It is difficult to determine the presence of maghemite $\gamma-\mathrm{Fe}_{2} \mathrm{O}_{3}$ (JCPDS 00-039-1346) from the XRD spectrum as the structures of magnetite $\mathrm{Fe}_{3} \mathrm{O}_{4}(\mathrm{a}=8.3515 \AA)$ and maghemite $\gamma-\mathrm{Fe}_{2} \mathrm{O}_{3}(8.3690 \AA)$ are the same and they have almost identical lattice parameters (Fang et al., 2012) (Cullity and Graham, 2011). But the presence of maghemite should be evident at higher angles. The peak at $74.11^{\circ}$ can be fit to a Lorentzian (shown in the inset to Figure 5.1.1) with the centre at $74.2^{\circ}$, attributed to the 440 peak of magnetite. In addition, there is no indication of a peak at $74.6^{\circ}$, which can be attributed to the (440) peak of maghemite. This is indicative of the concentration of maghemite being below the detection limit of $2 \%$. Maghemite is ferrimagnetic with a Curie temperature of $610 \mathrm{~K}$ (de Boer and Dekkers, 1996) but unlike magnetite it is semiconducting (Barakat, 2012). There are two low intensity peaks at $49.0^{\circ}$ and $52.40^{\circ}$ that can be attributed to small impurity phases of FeO (R-3, JCPDS pattern no. 039-1346) and iron, respectively. The lattice parameter for the magnetite from the sample is $8.3885 \AA$, which is comparable to that of the magnetite references, which is $8.3832 \AA$ (Cullity and Graham, 2011). This is greater than the lattice parameters of maghemite (8.3419 ̊).

Size and strain can broaden the diffraction peak. The Scherrer equation is used to determine the contribution from the average crystallite size (Koch et al., 2007).

$$
\beta_{\text {size }} \cos \theta=\frac{k \lambda}{D}
$$

where $\beta_{\text {size }}$ is the full-width half maximum from the $2 \theta$ plot, $\mathrm{k}$ is the shape factor, given by 0.9 (Langford and Wilson, 1978), and D is the average crystallite diameter for the particles. The apparent internal tensile strain caused by internal strains, regardless of the angle of reflection, is given by $\beta_{\text {str }} \cot \theta$ where $\beta_{\text {str }}$ is the 
integral breadth from strain. For a volume average in the $h k l$ direction, the strain is given by $\phi_{h k l}(0)=2 / \beta_{\text {str }} \cot \theta$. If assumptions are made of the stress being statistically isotropic on average, then the $\phi_{h k l}(0)=\frac{\varepsilon}{2}$, which gives us

$$
\beta_{\text {str }} \cot \theta=4 \varepsilon
$$

where $\varepsilon$ is the effective strain (Stokes and Wilson, 1944). The Williamson-Hall equation is the additive combination the contributions from the strain with plot can be used to determine the contributions from each. A Williamson-Hall plot of the magnetite peaks from Figure 5.1.1 was done using $\beta \cos \theta$ vs. $\sin \theta$ for the peaks at (111), (220), (311), (400), (511) and (440) and the results are shown in Figure 5.1.2. $\beta$ is the full width half maximum corrected for instrumental broadening from the $2 \theta$ plot, $\beta=\beta_{\text {str }}+\beta_{\text {size. The Williamson-Hall equation is }}$ given by (Williamson and Hall, 1953),

$$
\beta \cos \theta=\frac{k \lambda}{D}+4 \varepsilon \sin \theta
$$

Only magnetite peaks are taken. The plot is linear as shown by the fit, the slope of which can be used to obtain the strain, which is $0.17 \%$. The particles seem to be affected by strain and this does affect the particle size. The particle size, obtained from the intercept of the line is $162 \mathrm{~nm}$ and indicates that the average particles size is large. The FWHM is also greatly affected by the instrumental resolution, which is as large as 0.4 , assumed to be constant with the variation of angle. Strain reported for other magnetite nanoparticles synthesized by a conventional co-precipitation method has been reported to be $0.4 \%$ for $\sim 6 \mathrm{~nm}$ particles (Chaki et al., 2015). Similarly, magnetite nanoparticles $\sim 7 \mathrm{~nm}$ wide prepared by a sol-gel process have been reported to experience a strain of $1.2 \%$ (Sciancalepore et al., 2015). Magnetite particles synthesized by a chemical route have also reported negligible strain for $16 \mathrm{~nm}$ magnetite particles (Mürbe et al., 
2008). $0.3 \%$ for $15 \mathrm{~nm}$ magnetite particles (Rudolph et al., 2010). $0.02 \%$ for 31 nm magnetite particles (Jafari et al., 2014).

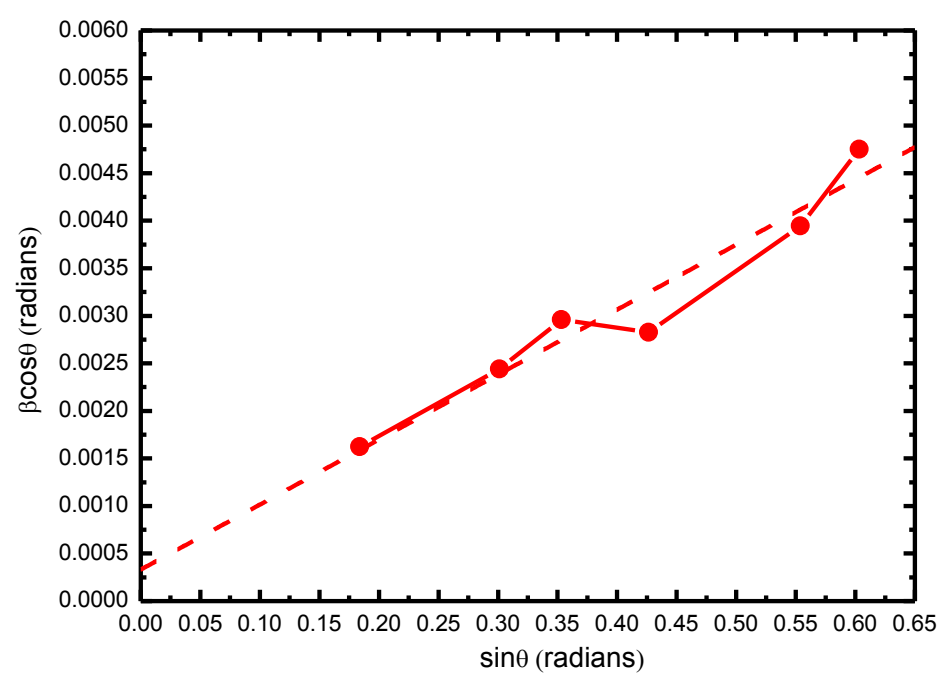

Figure 5.1.2. (colour online) Williamson-Hall plot of $\beta \times \cos \theta$ vs. $\sin \theta$ for the magnetite peaks in the XRD data with a linear fit (dashed line) to the data.

\subsubsection{Transmission electron microscopy}

The HRTEM images are shown in Figures 5.1.3a and 5.1.3b where it can be seen that there is a range of nanoparticle sizes and the nanoparticles are agglomerated. The agglomeration is likely to be partly due to magnetic dipole interactions between magnetic nanoparticles (Gass et al., 2006). There are numerous studies on nanoparticles; and many chemical studies focus on using surfactants to reduce agglomeration. When the particle sizes are above the superparamagnetic limit, the particles tend to aggregate because of the strong magnetic interactions. In some instances, although the particles sizes are below 
the superparamagnetic limit, the particles agglomerate and surfactants are needed to stabilize them (Chin et al., 2002; Gass et al., 2006).

The nanoparticle sizes in Figure 5.1.3a range from $7 \mathrm{~nm}$ to $75 \mathrm{~nm}$ where the average particle size is $\sim 25 \mathrm{~nm}$. There are large octahedral particles that are more evident in Figure 5.1.3b. Faceted magnetite nanoparticles have also been reported in studies where particles were found to be octahedral (Zhang et al., 2009) (Zhao and Duan, 2010) or cubic in shape (Dewi et al., 2014) for different methods of preparation. There are also a large number of smaller particles that appear to be spheres or truncated shapes without any faceting. Such an occurrence has also been reported for nanoparticles that are octahedral (Zhang et al., 2009). Using TEM to focus on different regions, it has become clear that the range in particles sizes is too varying to be understood by just one method to get an overall fraction of the particles under the superparamagnetic limit.

The SAED obtained from the powder is shown in Figure 5.1.3c. There are bright spots that form a series of rings, which is due to electron diffraction from a small number of orientated nanoparticles. Some of rings can be attributed to cubic magnetite as shown in Figure 5.1.3c by the red rings that are due to (111), (220), (311), (400), (511), and (440). Rhombohedral hematite is also observed where the (012), (104), (024), and (116) planes are indicated by green rings.

\subsubsection{Scanning electron microscopy}

An SEM image is shown in Figure 5.1.3d. There are some large particles up to $25 \mu \mathrm{m}$ as well as an agglomeration of much smaller particles. Thus, from Figure 5.1.3 it is apparent that the powder contains magnetite and hematite particles with sizes ranging from $7 \mathrm{~nm}$ to $25 \mu \mathrm{m}$. Although the fractional limit of the superparamagnetic particles in the overall sample cannot be determined, it is clear from the SEM and TEM measurements that there is a large fraction over the 
limit. The BEC image (not shown) indicates that there is uniform density of the particles, with the large particles being denser and metallic. The aggregation of the clusters is similar to that seen in the powder made by co-precipitation method in the next section. The strong magneto-interactions are evident.

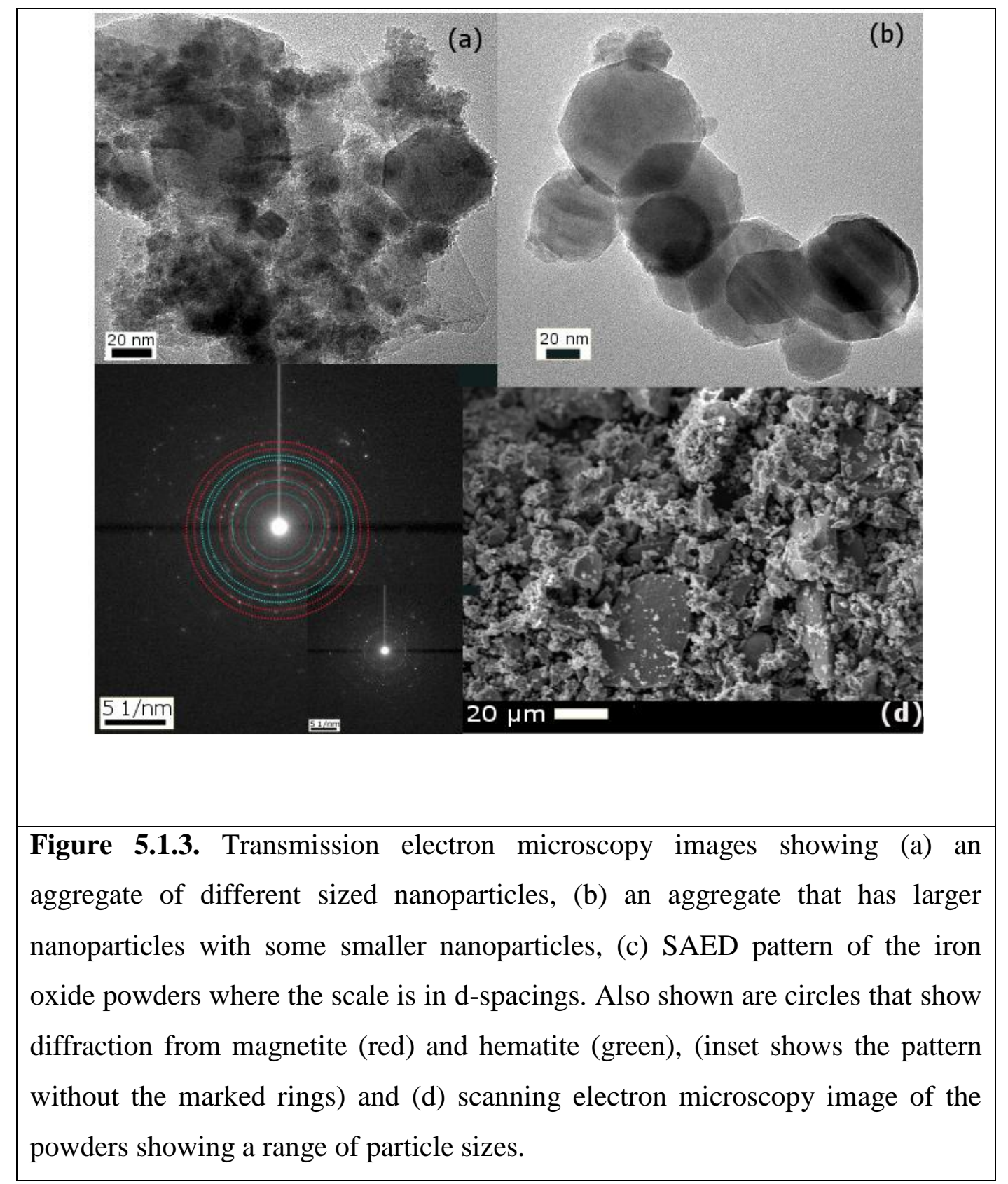




\subsubsection{Raman confocal microscopy measurements}

Raman spectroscopy measurements were made and the results from measurements on different parts of the powders can be seen in Figure 5.1.4. Figure 5.1.4a shows Raman spectra from a spot containing only magnetite. The three peaks at $310 \mathrm{~cm}^{-1}, 545 \mathrm{~cm}^{-1}$ and $670 \mathrm{~cm}^{-1}$ are due to the $\mathrm{T}_{2 \mathrm{~g}}, \mathrm{~T}_{3 \mathrm{~g}}$ and $\mathrm{A}_{1 \mathrm{~g}}$ allowed Raman modes of $\mathrm{Fe}_{3} \mathrm{O}_{4}$ (Jubb and Allen, 2010). The full width half maximum for the $A_{1 \mathrm{~g}}$ peak is $47 \mathrm{~cm}^{-1}$ and it is comparable to that reported in $\mathrm{Fe}_{3} \mathrm{O}_{4}$ thin films (Jubb and Allen, 2010). Raman measurements on another part of the powder show magnetite as well as a higher wavenumber shoulder on the $\mathrm{Fe}_{3} \mathrm{O}_{4} \mathrm{~A}_{1 \mathrm{~g}}$ peak as can be seen in Figure 5.1.4b. This can be attributed to a small fraction of maghemite and the maghemite $A_{1 g}$ allowed Raman mode at $700 \mathrm{~cm}^{-1}$ (Jubb and Allen, 2010) (Daou et al., 2006) (Slavov et al., 2010). Also shown in Figure 5.1.4b are the expected Raman shifts for the other two weaker magnetite peaks. This shows that there is some maghemite that is not observed in the XRD data. The appearance of maghemite in the Raman data but not in the XRD data is probably due to the larger Raman cross section for maghemite when compared with magnetite. Hematite is also seen in conjunction with magnetite in Figure $5.1 .4 \mathrm{c}$ at another spot. The peaks at $227 \mathrm{~cm}^{-1}, 248 \mathrm{~cm}^{-1}, 295 \mathrm{~cm}^{-1}, 303 \mathrm{~cm}^{-1}$, $413 \mathrm{~cm}^{-1}$ and $503 \mathrm{~cm}^{-1}$ can be attributed to the $A_{1 \mathrm{~g}}, E_{g}, E_{g}, E_{g}, E_{g}$ and $A_{1 g}$ allowed Raman modes from hematite (Chernyshova et al., 2007) (Owens and Orosz, 2006) (Chamritski and Burns, 2005). There is also a shoulder at $\sim 615 \mathrm{~cm}^{-1}$ that is due to the allowed hematite $A_{1 g}$ mode. Thus, the Raman data show that the main phase is magnetite and there is also some hematite and maghemite and there is a spatial distribution in the phase fractions. 


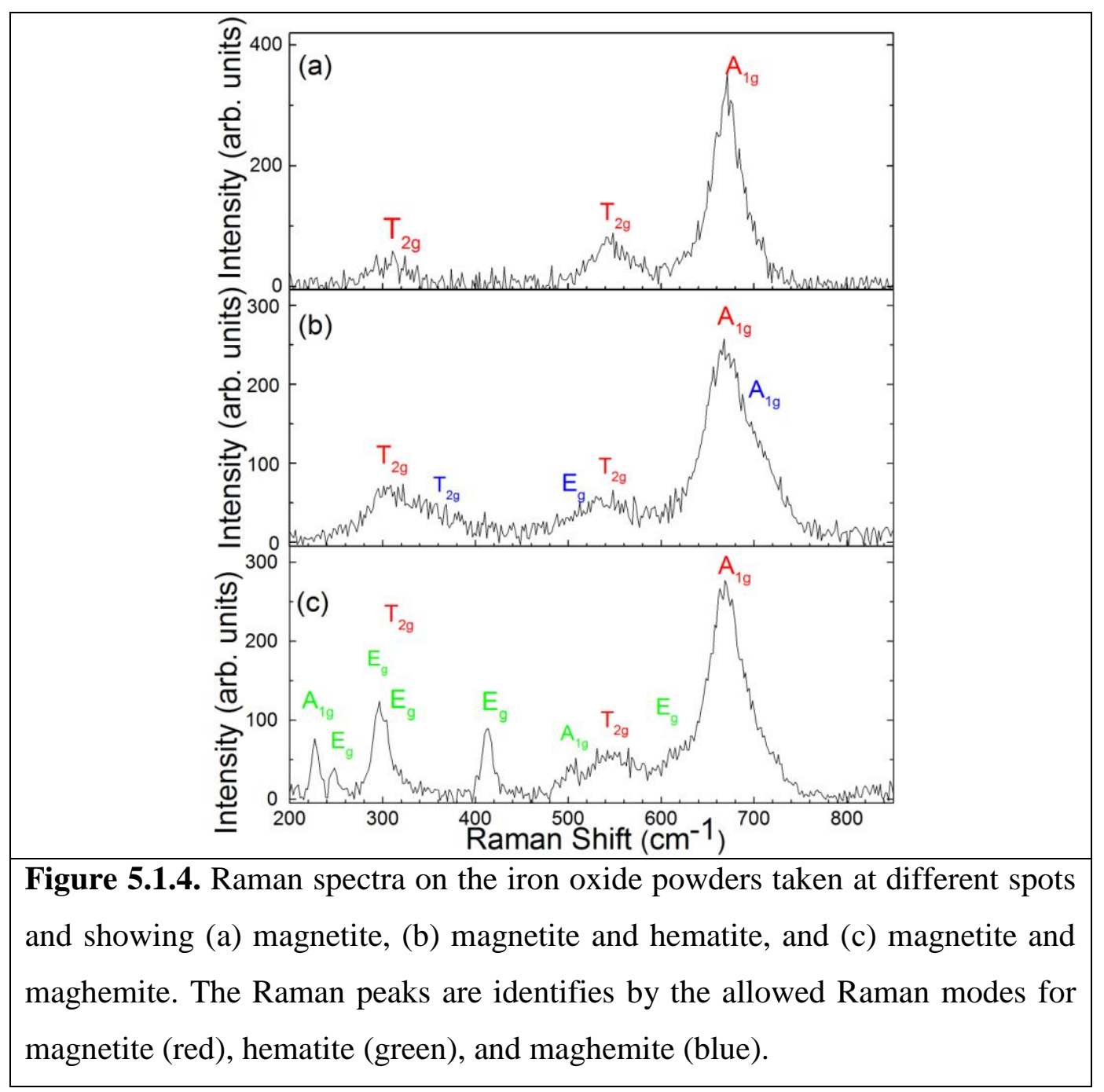

\subsubsection{Magnetization measurements}

The magnetization data are plotted in Figure 5.1.5 against the applied magnetic field, $\mathrm{B}$, at $10 \mathrm{~K}$ and $300 \mathrm{~K}$. The saturation magnetization is only slightly lower at $300 \mathrm{~K}$ than that at $10 \mathrm{~K}$, which indicates that the Curie temperature is far above room temperature. The expected value of saturation magnetization for bulk magnetite is $505 \mathrm{kA} / \mathrm{m}$ (Cullity and Graham, 2011; Smit and Wijn, 1959) and is also shown in Figure 5.1.5. The saturation moment for our 
iron oxide powder is lower and it is $375 \mathrm{kA} / \mathrm{m}$ at $10 \mathrm{~K}$. The lower value of the measured saturation moment is likely to be due to the antiferromagnetic hematite phase fraction. From the reduced measured saturation it can be estimated that $26 \%$ of the powder contains hematite. $(26 \%$ is from the decrease in the saturation magnetization value as compared to magnetite. Magnetite has a magnetization saturation value of $90 \mathrm{emu} / \mathrm{g}$, which is $26 \%$ lower than the value of $80 \mathrm{emu} / \mathrm{g}$ seen for this iron oxide powder.)

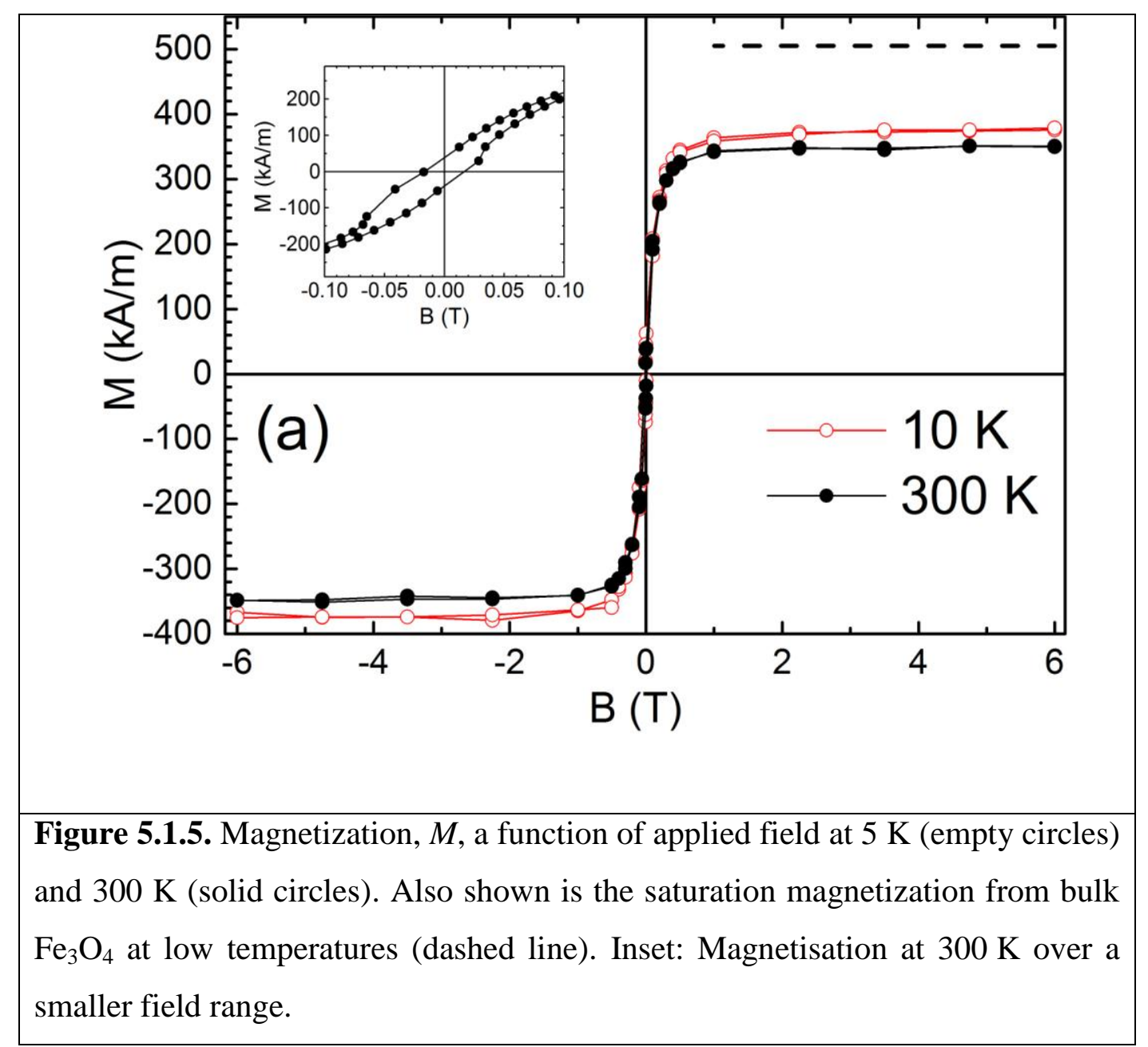


A field loop was also done over a smaller field range at $300 \mathrm{~K}$ and the results can be seen in the inset to Figure 5.1.5. There is some hysteresis at $300 \mathrm{~K}$ and the coercive field is $17 \mathrm{mT}$. This is expected because most of the particles are large and hence the hysteresis arises from these non-superparamagnetic particles of radii that are larger than the superparamagnetic diameter (Lin et al., 2006).

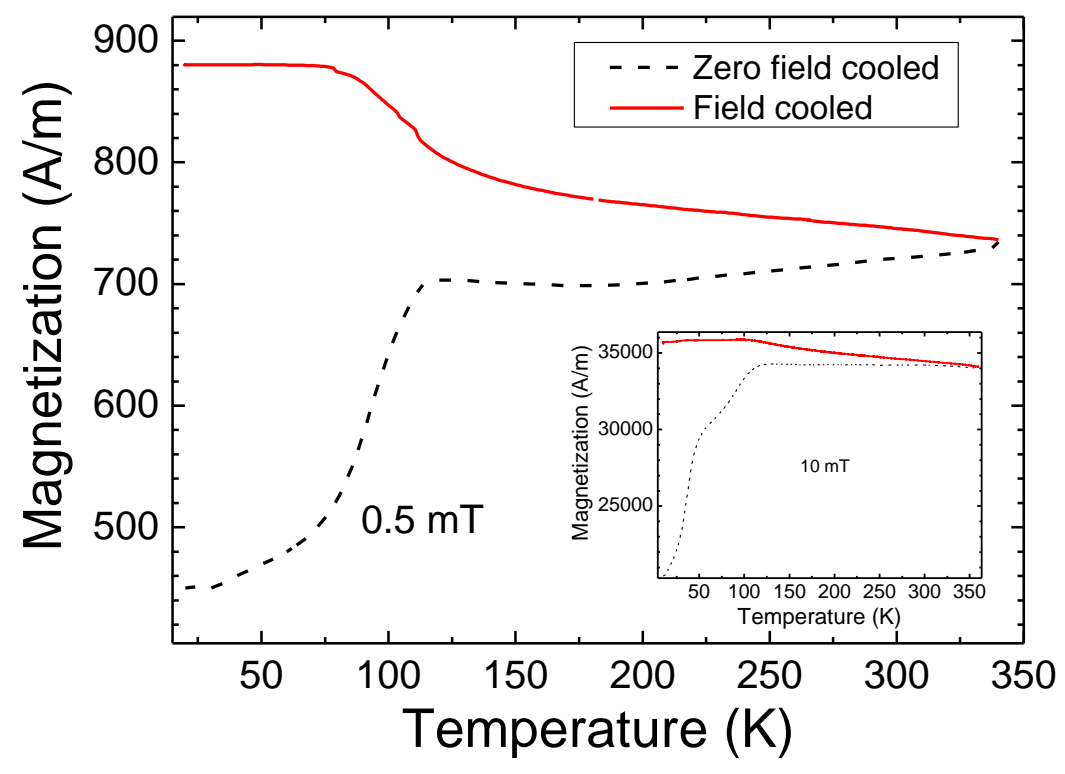

Figure 5.1.6. Zero-field-cooled (solid curve) and field-cooled (dashed curve) magnetization curves for the iron oxide powder at $0.5 \mathrm{mT}$ and at $10 \mathrm{mT}$ (inset).

Zero-field-cooled (ZFC) and field-cooled (FC) magnetization measurements were made at $0.5 \mathrm{mT}$ and $10 \mathrm{mT}$ and the results can be seen in Figure 5.1.6 and the inset within this figure. In the $0.5 \mathrm{mT}$ data, there is irreversibility up to the highest measured temperature, which is consistent with the absence of superparamagnetism. The ZFC and the FC magnetization show a large decrease below $120 \mathrm{~K}$ that can be attributed to the Verwey transition in magnetite (Senn et al., 2011),(Ziese and Blythe, 2000). The Verwey transition is at the 
expected temperature for the bulk $\mathrm{Fe}_{3} \mathrm{O}_{4}$ stoichiometry. It is not observed in the ZFC-FC data from small magnetite nanoparticles but it is reported in faceted $\mathrm{Fe}_{3} \mathrm{O}_{4}$ nanoparticles (Mitra et al., 2014),(Ma et al., 2014). From the inset, there is a further decrease in the ZFC magnetization below $\sim 50 \mathrm{~K}$ for a higher applied magnetic field of $10 \mathrm{mT}$ and the origin cannot be attributed to any magnetic or structural transition in magnetite, maghemite, or hematite. This transition has been seen for magnetite nanoparticles that are $30-50 \mathrm{~nm}$ wide and been attributed to a spin-glass transition (Lopez Maldonado et al., 2014). However, this is unlikely the case in these iron oxide powders because of the low fraction of nanoparticles. It is not seen in the $0.5 \mathrm{mT}$ data and hence it is clear that the transition is not intrinsic to the material. It is possible that it is due to domain wall pinning.

There have been very few studies of the temperature dependence of the saturation magnetization in bulk $\mathrm{Fe}_{3} \mathrm{O}_{4}$ from low temperatures up to $300 \mathrm{~K}$. One study showed that there is a change in the temperature dependence of the saturation magnetization below the Verwey transition temperature, $T_{\mathrm{V}}$, in a $\mathrm{Fe}_{3} \mathrm{O}_{4}$ single crystal (Aragón, 1992). This also occurs in the $\mathrm{M}_{\mathrm{s}}(\mathrm{T})$ data. It is clearer in the inset to Figure 5.1 .7 where $\left[M_{s}(0)-M_{s}(T)\right] / M_{s}(0)$ is plotted and there is a departure from the high temperature power law behaviour below $127 \mathrm{~K}$. This departure has not been reported in small $4 \mathrm{~nm}$ diameter $\mathrm{Fe}_{3} \mathrm{O}_{4}$ nanoparticles where the Verwey transition is not observed (Goya et al., 2003). $M_{s}(T)$ was fitted to Equation $2.11, M_{\mathrm{s}}=M_{\mathrm{s}}(0)\left[1-B \times T^{\beta}\right]$, over the full temperature range up to 300 $\mathrm{K}$ and $\beta=3 / 2$ was found (Goya et al., 2003). One study on magnetite nanoparticles reported $\beta=2$ but there were few data points and the data range was limited to $\sim 100 \mathrm{~K}$ to just above $300 \mathrm{~K}$ (Ortega et al., 2010). 


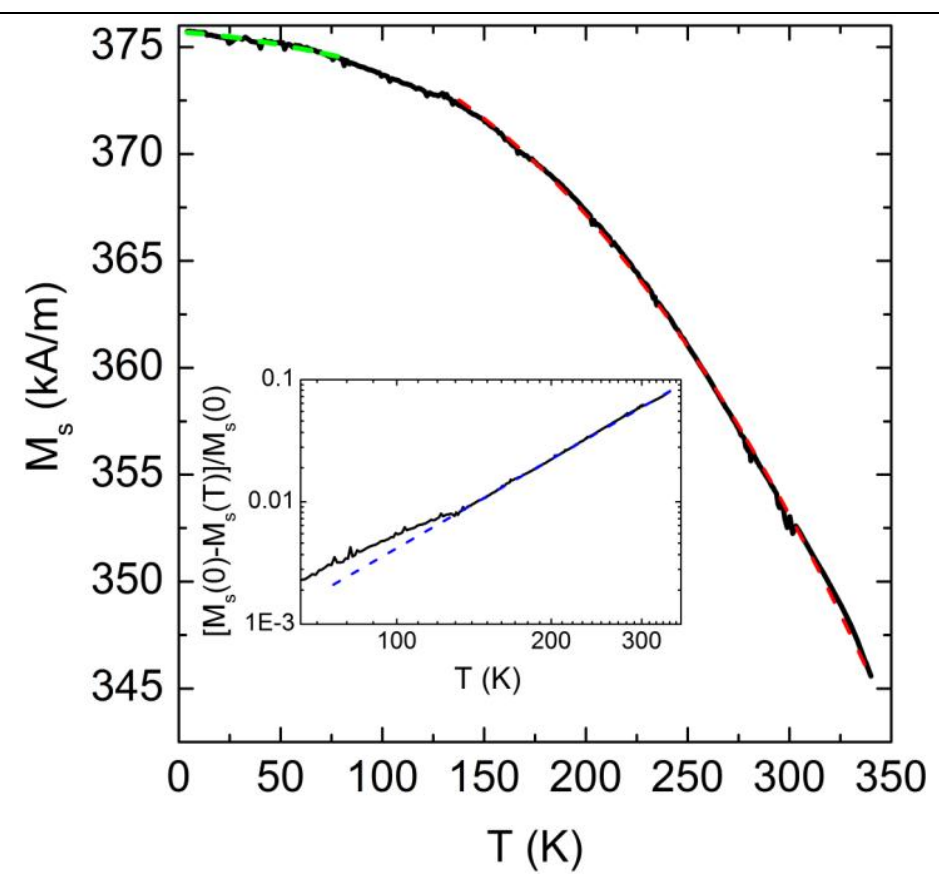

Figure 5.1.7. Saturation magnetization, $M_{\mathrm{s}}$, as a function of temperature at an applied field of $6 \mathrm{~T}$ (solid curve). Also shown are fits to Equation 2.11 in the low and high temperature regions (dashed curves). Inset: Log plot of $\left[\mathrm{M}_{\mathrm{s}}(\mathrm{T})-\right.$ $\left.\mathrm{M}_{\mathrm{s}}(0)\right] / \mathrm{M}_{\mathrm{s}}(0)$ as a function of the log of the temperature (solid curve) showing a linear dependence in the temperature range of $130 \mathrm{~K}$ to $350 \mathrm{~K}$ (dashed curve).

$M_{s}(T)$ data was fitted above $T_{\mathrm{V}}$ to Equation 2.11 (see Figure 5.1.7) and it is found that $\beta=2.26$ and $B=1.6 \times 10^{-7} \mathrm{~K}^{2.26} . \beta$ is higher than that reported in $\mathrm{Fe}_{3} \mathrm{O}_{4}$ nanoparticle studies (Goya et al., 2003). It is significantly smaller than that in a stoichiometric $\mathrm{Fe}_{3} \mathrm{O}_{4}$ single crystal (Aragón, 1992) where analysis of the saturation magnetization data gives $\beta=3.0$. The lower $\beta$ found in polycrystalline $\mathrm{Fe}_{3} \mathrm{O}_{4}$ made by the arc-discharge method may be due to averaging of spin-waves over all crystallographic directions that does not occur in the single crystal.

At low temperatures it is possible to fit $M_{s}(T)$ to Equation 2.11 with $\beta=3 / 2$ (see Figure 5.1.7) and this has also been reported from a magnetization study on a 
$\mathrm{Fe}_{3} \mathrm{O}_{4}$ single crystal (Aragón, 1992). $\beta=3 / 2$ at low temperatures is predicted in $\mathrm{Fe}_{3} \mathrm{O}_{4}$ if the exchange interactions between ions in the same sublattice are weak when compared with exchange interactions between ions in different sublattices and in the absence of significant excitation of optic branch spin-waves (Aragón, 1992). In this case $B$ can be used to estimate the exchange energy, $J_{a b}$, between the spins on the tetrahedral and octahedral Fe sites (Aragón, 1992). Using the experimental $B=4.4 \times 10^{-6} \mathrm{~K}^{3 / 2}$ it is possible to show that $J_{a b} / k_{\mathrm{B}}=42 \mathrm{~K}$. This can be compared with a lower $J_{a b} / k_{\mathrm{B}}$ of $23 \mathrm{~K}$ found in a $\mathrm{Fe}_{3} \mathrm{O}_{4}$ single crystal (Aragón, 1992). The higher $J_{a b} / k_{\mathrm{B}}$ found in the unorientated powder made by the arcdischarge method may be due to averaging over all crystallographic directions.

\subsubsection{Magnetoresistance measurements}

Magnetoresistance measurements were performed on a pressed pellet and the resultant magnetoresistance is plotted in Figure 5.1.8a against the applied magnetic field for different temperatures. The magnetoresistance, MR, is defined as $\mathrm{MR}=[R(B)-R(0)] / R(0)$ where $R(B)$ is the resistance at an applied magnetic field $B$ and $R(0)$ is the resistance for zero applied magnetic field. The magnetoresistance reaches $-9.5 \%$ at $120 \mathrm{~K}$ and for high magnetic fields. Similar magnetoresistance values and magnetic field dependences of the magnetoresistance have previously been reported in $\mathrm{Fe}_{3} \mathrm{O}_{4}$ nanocomposites (Kumar et al., 2014; H. Liu et al., 2003; Venkatesan et al., 2003) and attributed to spin-tunnelling between $\mathrm{Fe}_{3} \mathrm{O}_{4}$ particles (K. Liu et al., 2003). Thus, the magnetoresistance in our powder is due to intergrain spin-tunnelling and the semiconducting hematite fraction is below the percolation limit and does not significantly contribute to the electrical resistance.

Magnetoresistance measurements performed over a smaller field range with a sweep rate of $1 \mathrm{mT} / \mathrm{s}$ are shown in Figure 5.1.8b. The coercivity is evident from the low field measurements as there is a small peak in the magnetoresistance 
at $0.029 \mathrm{~T}$ and $0.003138 \mathrm{~T}$. This value of coercivity is much more than the value seen in the field dependant magnetisation. This is typical of materials with grain boundary regions where the intergrain tunnelling magnetoresistance effect arises from spin dependant scattering at the grain boundaries (Serrate et al., 2005b). This effect results in a very pronounced low field magnetoresistance. Lattice shifts across the lattice parameters can also cause such spin dependant scattering which has been reported in magnetite thin films (Eerenstein et al. 2002). However, in this case the magnetoresistance depends on the magnetic field and is ruled out in this case (Serrate et al., 2007).

For granular systems, the intergrain spin-tunnelling magnetoresistance can be written as (Inoue and Maekawa, 1996), from equation 2.14, $\operatorname{MR}(B)=-\frac{P^{2} m(B)^{2}}{1+P^{2} m(B)^{2}}$, where $m(B)$ is the reduced magnetization and $P$ is the fraction of spin polarized carriers.

Bulk magnetite has $P=80 \%$ at $300 \mathrm{~K}$ and it is $6 \%$ higher at very low temperatures (Dedkov et al., 2002) and hence it is expected that $\mathrm{MR}=-42 \%$ in granular $\mathrm{Fe}_{3} \mathrm{O}_{4}$ at low temperatures and high magnetic fields. The magnetoresistance that is measured here is clearly less than this and the magnetoresistance does not saturate even at $8 \mathrm{~T}$ (Figure 5.1.8a) while the magnetization has saturated above $1 \mathrm{~T}$ (Figure 5.1.5). This has been observed before in granular systems and it has been attributed to a spin disordered region at the grain boundaries as shown in Figure 5.1.9 (Serrate et al., 2005b). 


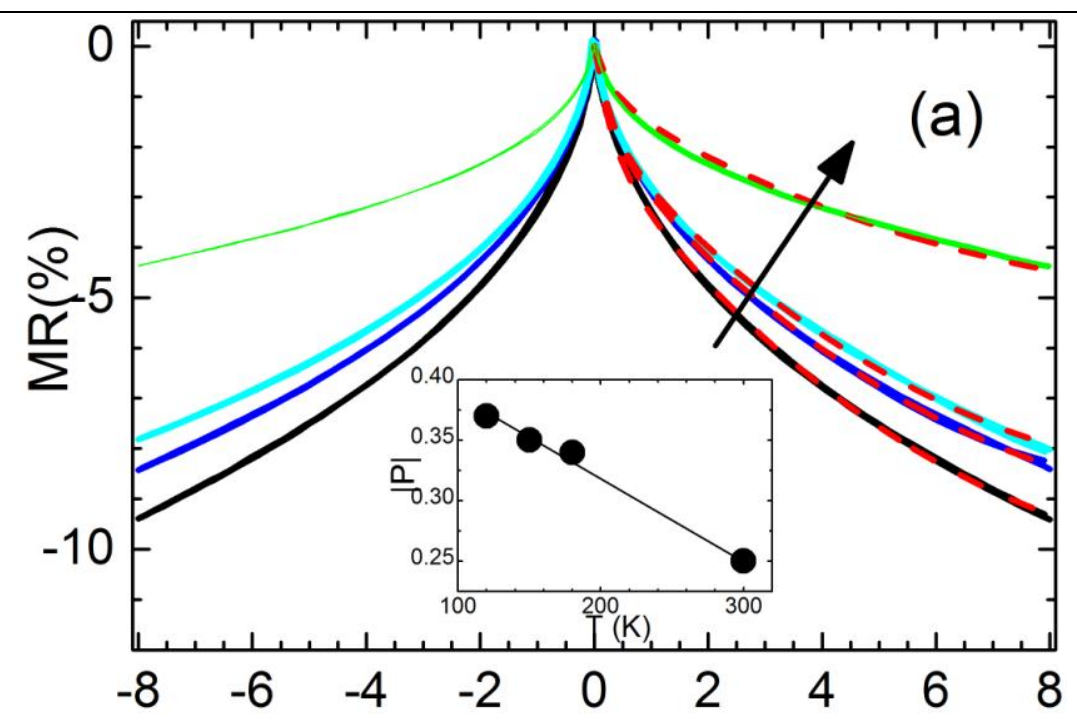

Field (T)

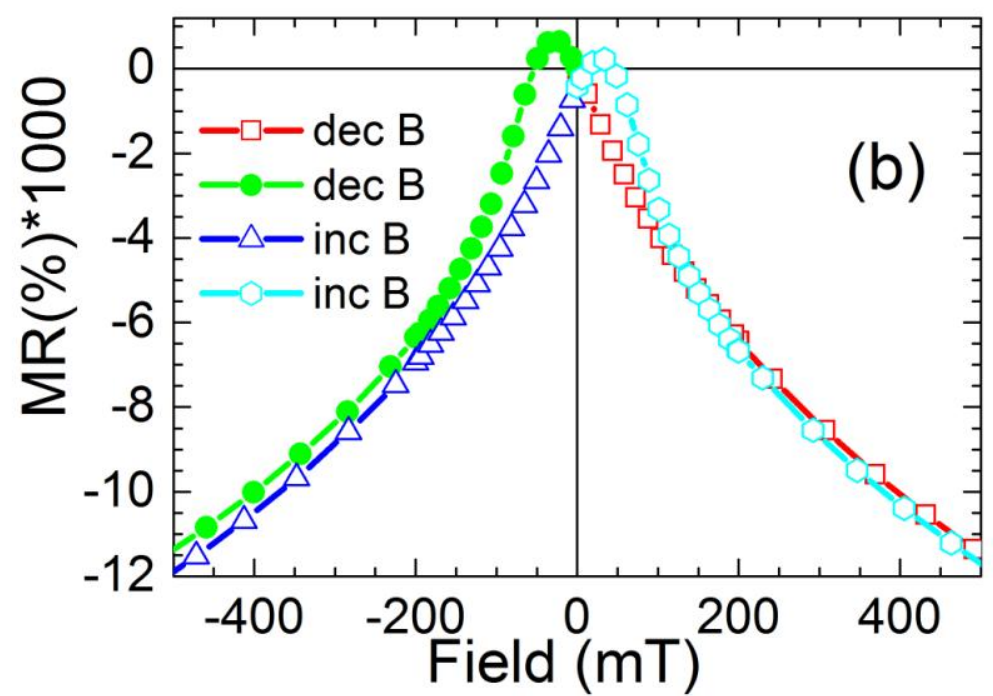

Figure 5.1.8. (colour online) (a) Magnetoresistance plotted against the applied magnetic field at $120 \mathrm{~K}, 150 \mathrm{~K}, 180 \mathrm{~K}$, and $300 \mathrm{~K}$ (solid curves). The arrow shows increasing temperature. Also shown are fits to the data using Equations 2.14 and 5.2 (dashed curves). Inset: Plot of the fitted degree of electronic polarization, $P$, against temperature. The solid line is a guide to the eye. (b) Plot of the low-field magnetoresistance against the applied field at $300 \mathrm{~K}$ showing the magnetoresistance for decreasing and increasing B. 
The disordered region will degrade the spin polarized current and reduce the magnetoresistance. The resultant magnetoresistance has been previously modelled by replacing $m(B)$ in Equation 2.14 with $m_{\mathrm{gb}}(B)$ where $m_{\mathrm{gb}}(B)$ is the reduced magnetization from the spin-disordered grain boundary region (Serrate et al., 2005b). Different functions have been used to model $m_{\mathrm{gb}}(B)$ and the magnetoresistance (Inoue and Maekawa, 1996; K. Liu et al., 2003; Park et al., 2005). It is possible to use a phenomenological $m_{\mathrm{gb}}(B)$ that can be written as,

$$
m_{\mathrm{gb}}(B)=\gamma \tanh \left(B / B_{0}\right)+(1-\gamma) \exp (-a / \sqrt{B}),
$$

where $\gamma, B_{0}$, and $a$ are fitting parameters. At high fields, $m_{\mathrm{gb}}(B) \sim 1-\mathrm{a} / \sqrt{B}$. This has been expected reduced magnetization for a spin-glass with weak random anisotropy (Serrate et al., 2005b).

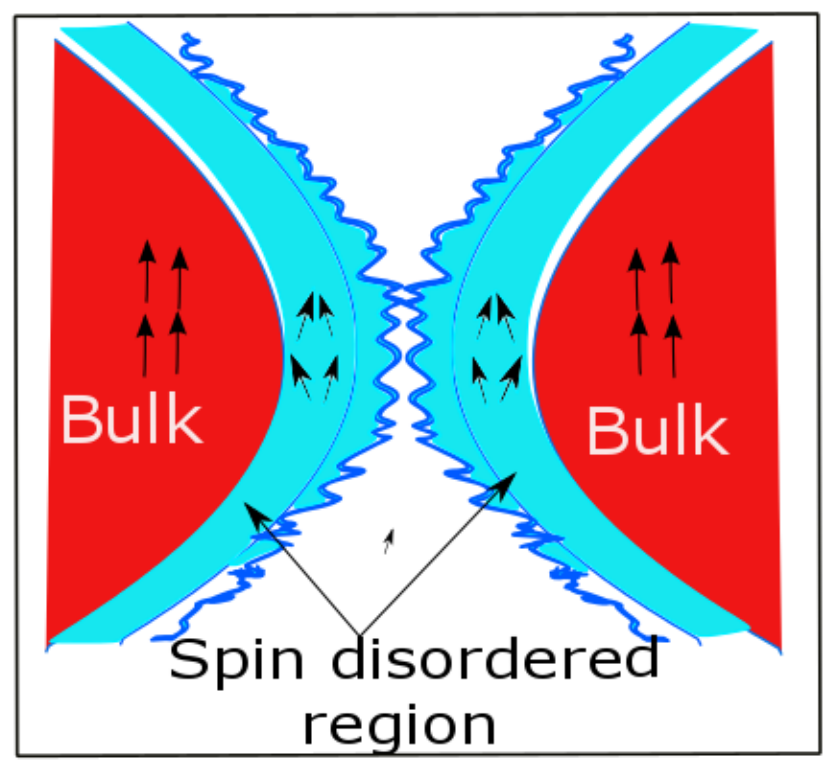

Figure 5.1.9 Schematic diagram of the model of the grain boundary region with disordered spins and ordered spins in the bulk. 
In Figure 5.1.8, Equations 2.14 and 5.2 give a good fit to the magnetoresistance data for all temperatures with $\gamma=0.37, a=0.34 \mathrm{~T}^{1 / 2}$, and $\mathrm{B}_{0}=6.9 \mathrm{~T}$. The fitted $|P|$ is plotted in Figure $5.1 .8 \mathrm{~b}$ where it can be seen that it is as high as $37 \%$ at $120 \mathrm{~K}$ and reduces to $25 \%$ at $300 \mathrm{~K}$. These values are clearly lower than reported in bulk $\mathrm{Fe}_{3} \mathrm{O}_{4}$, which is consistent with a reduction in the spin polarization in the spin disordered region at the grain boundaries. The change in $|P|$ between $120 \mathrm{~K}$ and $300 \mathrm{~K}$ is larger than that measured in bulk $\mathrm{Fe}_{3} \mathrm{O}_{4}$ where $|P|$ is only reduced by $6 \%$ between $0 \mathrm{~K}$ and $300 \mathrm{~K}$ due to spin wave excitation (Dedkov et al., 2002). A significant reduction in $|P|$ has been reported in other granular spin polarized systems and shows that there is significant spinscattering in the disordered region that increases with increasing temperature (Cerri et al., 1983; Garcia et al., 2004; Shang et al., 1998).

It can be seen in Figure 5.1.10 that the electrical resistance strongly increases as the temperature is reduced. Furthermore, the resistance as a function of temperature, $\mathrm{R}(\mathrm{T})$, can be fitted to,

$$
R(T)=R_{0} \exp \left(\left(T_{0} / T\right)^{\alpha}\right)
$$

where $T_{0}=38800 \mathrm{~K}$ and $\alpha=1 / 2$. This temperature dependence has been reported in disordered compounds and attributed to variable range hopping about localized states at the Fermi energy in the presence of Coulomb interactions (Efros and Shklovskii, 1975). It is unlikely that this occurs in our granular sample because it is not compatible with spin-dependent tunnelling that provides a good description of the magnetoresistance data. 


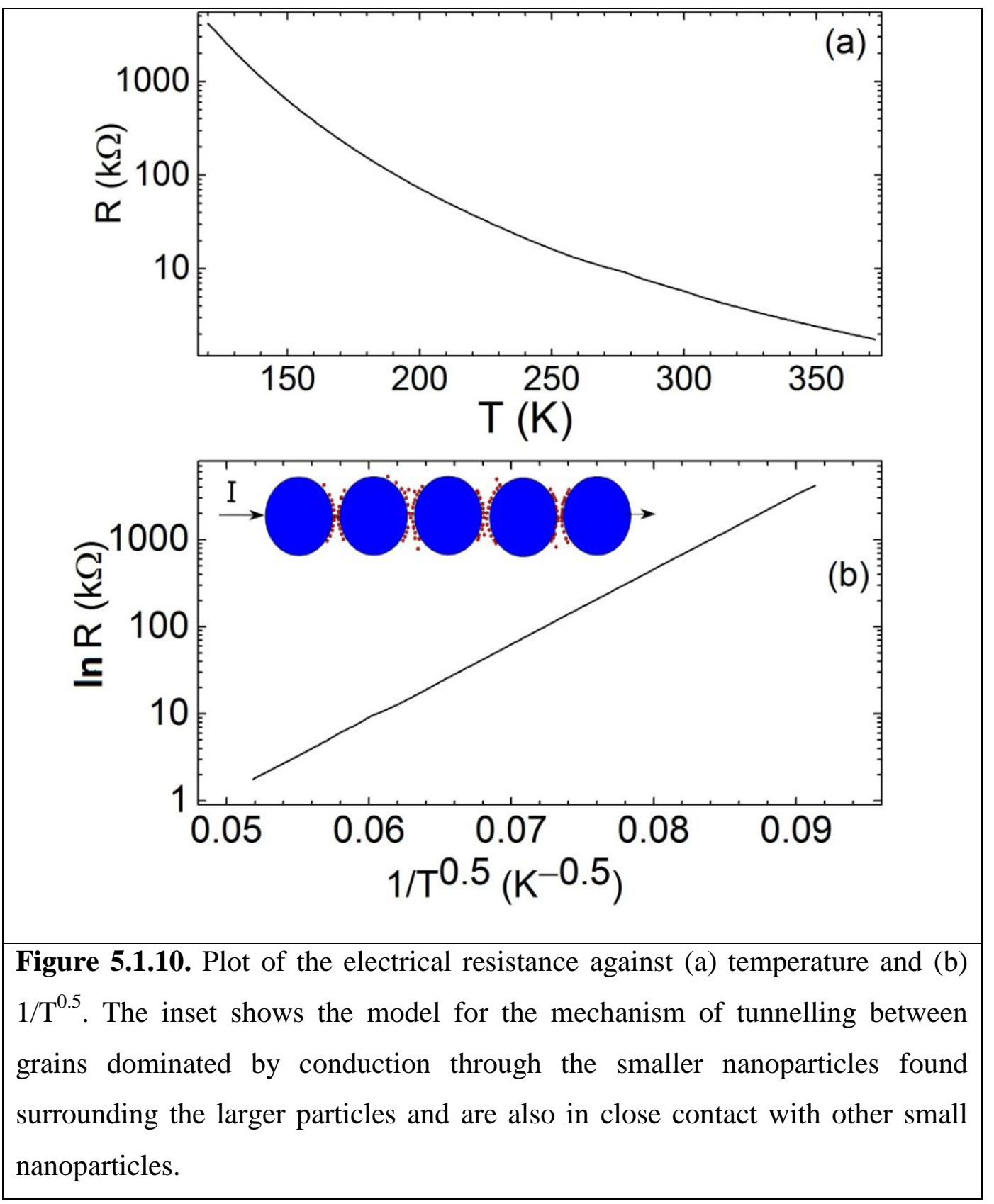

A value of $\alpha=1 / 2$ has been reported in $\mathrm{Fe}_{3} \mathrm{O}_{4}$ nanoparticle composites where there is spin-dependent tunnelling (H. Liu et al., 2003; Park et al., 2005; Serrate et al., 2005a; Song et al., 2006). It can occur because the electron tunnelling process leads to a positive charge on one nanoparticle and a negative 
charge on the other nanoparticle. This introduces an electrostatic charging energy, $E_{\mathrm{c}}$, for each tunnelling electron that can be written as $E_{\mathrm{c}}=\left[e^{2} / d\right] f(s / d)$ where $d$ is the particle size, $s$ is the particle separation, $e$ is the electronic charge, and $f(s / d)$ is a function that depends on the particle shape (Sheng et al., 1973). If $s / d$ is the same value for different regions in the nanocomposite then $s \times E_{c}$ is a constant. Using a steepest decent method it is then possible to show that the resultant tunnelling electrical resistance can be written (Sheng et al., 1973),

$$
R(T, B)=R_{0} \exp \left[2\left[\kappa C_{0} /\left[k_{\mathrm{B}} T\right]\right]^{1 / 2}\right]
$$

where $C_{0}=2 \chi s_{0} E_{c}, \quad \kappa=\left[2 m *\left(\phi-E_{\mathrm{F}}\right) / \hbar^{2}\right]^{1 / 2}, s_{0}$ is the average $s, \phi$ is the effective barrier height, $m^{*}$ is the effective mass of the electrons, $E_{\mathrm{F}}$ is the Fermi energy, $\hbar$ is Planck's constant divided by $2 \pi$, and $R_{0}$ is a constant. In the presence of spin-dependent tunnelling in granular magnetic samples, $R_{0}$ in equation 5.4 can be rewritten as $R_{0}(B, T)=R_{01} /\left[1+P(T)^{2} m(B, T)\right]$ where $R_{01}$ is independent of temperature (Inoue and Maekawa, 1996). However, the temperature dependence of the electrical resistance is still dominated by the exponential factor in Equation 5.4. It is unlikely that Equation 5.4 is applicable for conducting particles with $d$ on the micron length scale due to rapid charge redistribution that would result in an electrostatic charging energy that would be too small to measure. In our case there is a range of particle sizes from $7 \mathrm{~nm}$ to $25 \mu \mathrm{m}$. Thus, electrostatic charging is only possible in our powders if the percolation pathway is dominated by spindependent tunnelling between the small particles. This could occur if the larger metallic $\mathrm{Fe}_{3} \mathrm{O}_{4}$ particles were surrounded by the small $\mathrm{Fe}_{3} \mathrm{O}_{4}$ particles as shown in the inset of Figure 5.1.10b. In this scenario, the percolation pathway would be through large $\mathrm{Fe}_{3} \mathrm{O}_{4}$ particles with low resistances and also through small $\mathrm{Fe}_{3} \mathrm{O}_{4}$ particles where the spin tunnelling between the small $\mathrm{Fe}_{3} \mathrm{O}_{4}$ particles would dominate the resistance. $\kappa C_{0} / k_{\mathrm{B}}$ obtained in our powders by fitting the data in 
Figure 5.1.10b to equation $5.2(9700 \mathrm{~K})$ is in the range of that observed in other studies on $\mathrm{Fe}_{3} \mathrm{O}_{4}$ powders (Park et al., 2005; Serrate et al., 2005b). Further structural and theoretical study is required to definitively ascertain the origin of the $\alpha=1 / 2$ temperature dependent resistance that is beyond the scope of the present study.

\subsubsection{Conclusions}

In conclusion, magnetite powders can be made using a new arc-discharge method with an iron anode and a partial oxygen atmosphere. XRD and SAED measurements show that the resulting powder is predominantly ferromagnetic magnetite with some antiferromagnetic hematite $\left(\alpha-\mathrm{Fe}_{2} \mathrm{O}_{3}\right)$. There is also some ferromagnetic maghemite $\left(\gamma-\mathrm{Fe}_{2} \mathrm{O}_{3}\right)$ that is seen in the Raman data but not the XRD data where measurements taken at different spots showed that iron oxide phases are inhomogeneously distributed in the powder. The powder contained nanoparticles as small as $7 \mathrm{~nm}$ as well as large particles with sizes up to $25 \mu \mathrm{m}$. The magnetization saturation is less than that observed for the bulk magnetite, which is due to the presence of $\sim 26 \%$ of antiferromagnetic hematite. The temperature dependence of $M_{s}(T)$ changes at $T_{\mathrm{V}}$, which has also been reported in a $\mathrm{Fe}_{3} \mathrm{O}_{4}$ single crystal. $M_{s}(T)$ is observed to have a power law dependence above $T_{\mathrm{V}}$ where $\beta=2.23$. At low temperature $M_{s}(T)$ can be fitted with $\beta=3 / 2$ and $J_{a b} / k_{\mathrm{B}}=42$ K. Pressed pellets of the powder display a magnetoresistance that reaches $-9.5 \%$ at $120 \mathrm{~K}$ and is dominated by the spin-dependant tunnelling between the magnetite grains. This value is significantly greater than that observed for permalloy nanoparticles synthesized using the same arc-discharge method owing in part to the higher intrinsic degree of electronic polarization in $\mathrm{Fe}_{3} \mathrm{O}_{4}$. A phenomenological model was used to fit the magnetoresistance and find that the fitted magnitude of the electronic spin polarization is $37 \%$ at $120 \mathrm{~K}$ and decreases with increasing temperature. This is less than the maximum of $80 \%$ expected at 
$300 \mathrm{~K}$ because of a reduction in the spin polarized current near the grain boundaries in a spin-disordered region. The temperature dependence of the resistance is exponential and has an exponent of $1 / 2$. This exponent may be due to small nanoparticles coating larger grains and the effect of electrostatic charging during spin-dependent tunnelling between the nanoparticles. 


\subsection{Magnetite nanoparticles synthesized by a co-precipitation method}

This section looks in detail into magnetite made by a co-precipitation method. The aim is to study the magnetic properties and a model the magnetoresistance magnetoresistance in order to study the contribution of the spin disorder and how particle sizes or shape affect the magnetic properties.

\subsubsection{Synthesis technique}

An $8.5 \mathrm{M}$ solution of $25 \mathrm{~mL}$ of $\mathrm{NaOH}$ was prepared. $6 \mathrm{~g}$ of $\mathrm{FeCl}_{2}$ and $12 \mathrm{~g}$ of $\mathrm{FeCl}_{3}$ were dissolved in $25 \mathrm{~mL}$ de-ionized water. Then, the $25 \mathrm{~mL} \mathrm{NaOH}$ solution was added to the mixture of iron salts under mechanical stirring for 30 mins at room temperature at $1100 \mathrm{rpm}$. After the reaction, the precipitate was washed twice with distilled water by centrifugation for $5400 \mathrm{~s}$ (Karaagac and Kockar, 2012). Structural properties were investigated by XRD using Co $\mathrm{K}_{\alpha}(\lambda=$ $1.74 \AA$ A) radiation. TEM was employed to examine the microstructural properties of the magnetite nanoparticles. SQUID magnetometer from Quantum Design was used to investigate the magnetic properties of the nanoparticles. Magnetization measurements were made at $5 \mathrm{~K}$ and $300 \mathrm{~K}$ and temperature-dependent magnetization measurements were performed with stable temperatures. Magnetoresistance measurements were made on a pressed pellet using the fourterminal method in a Quantum Design PPMS and with the current parallel or perpendicular to the applied magnetic field. The four-terminal magnetoresistance measurements were made by first evaporating silver electrodes and then using silver epoxy bonded contact wires. The electrical resistance was measured as a function of temperature with a sweep of the temperature at a rate of $1 \mathrm{~K} / \mathrm{min}$. Magnetite particles made by a chemical process described by Schwertmann and Cornell (2008) were used as a reference powder for XRD measurements 
(Schwertmann and Cornell, 2008). Maghemite and hematite particles made by a chemical synthesis process were also used as reference powders for Raman characterization (Schwertmann and Cornell, 2008).

\subsubsection{Structural investigation}

\subsubsection{X-ray diffraction}

XRD data from the powder synthesized are shown in Figure 5.2.1. The XRD peak positions are consistent with face centred cubic structure of magnetite $(\operatorname{Fd} \overline{3} \mathrm{~m})$. XRD data from the powder synthesized and the corresponding Miller indices for each peak are shown in Figure 5.2.1. Also shown are the peaks to a magnetite reference powder that was made using a similar co-precipitation method. The particles that were synthesized had a larger crystallite size (Schwertmann and Cornell, 2008). The XRD peaks correspond to magnetite (00039-1346) with no indication of hematite (JCPDS 033-0664). The lattice parameters for maghemite (JCPDS 00-039-1346) are very close to that of maghemite $\left(\gamma-\mathrm{Fe}_{2} \mathrm{O}_{3}\right)$ and it is difficult to rule out the presence of maghemite in the powder. The broad peaks of the magnetite powder in comparison to the magnetite reference indicate that the magnetite powder that was synthesized has very small crystallite sizes. Using the Scherrer equation (Azaroff and Buerger, 1958), given by

$$
D=\frac{0.9 \lambda}{\beta \cos \theta}
$$

where $\lambda$ is the wavelength of the cobalt radiation $(1.74 \AA), \theta$ is the Bragg angle and $\beta$ is the full width half maximum corrected for instrumental broadening, the average crystallite size was determined to be $\sim 14 \mathrm{~nm}$. The lattice spacing derived from the most intensive peak at $41.465^{\circ}$ is $4.865 \AA$, which corresponds closely to 
$4.922 \AA$, which was seen in the magnetite reference powder. This corresponds to the (311) peak of magnetite. No other peaks from iron or other phases of iron oxide are seen. The average crystallite size seen from a similar co-precipitation method report similar sizes of nanocrystals that are on average $\sim 5 \mathrm{~nm}$ with a range from $\sim 2 \mathrm{~nm}$ to $\sim 7 \mathrm{~nm}$ (Cai and Wan, 2007; Sun et al., 2004; Wu et al., 2007). The average crystallite size for the magnetite reference powder was found to be $60 \mathrm{~nm}$.

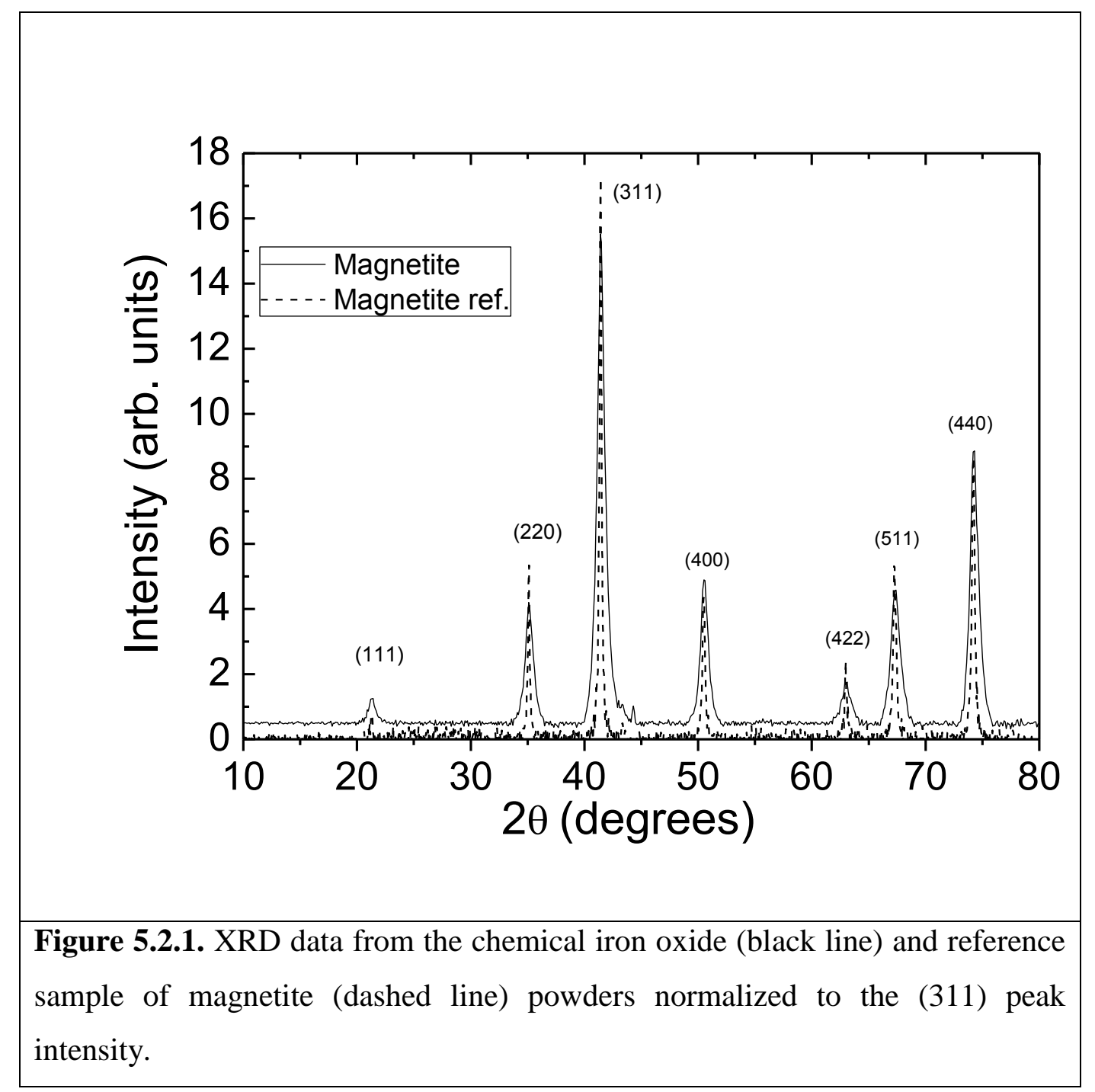


A Williamson-Hall plot of the peaks was done using $\beta \cos \theta$ vs. $\sin \theta$ for the peaks at (111), (220), (311), (400), (422), (511) and (440) were plotted and the results are shown in Figure 5.2.2 (see overleaf), where the Williamson-Hall equation is given by (Williamson and Hall, 1953) $\beta \cos \theta=\frac{k \lambda}{D}+4 \varepsilon \sin \theta$ (Equation 5.1) where $\beta$ is the full-width half maximum from the $2 \theta$ plot and $k$ is the shape factor, given by $0.9, D$ is the average crystallite diameter for the particles and $\varepsilon$ is the effective strain. The slope of the line for Williamson-Hall plot of the synthesized powder is zero and hence strain is negligibly low. Hence the size determined by the Scherrer equation remains true. This is similar to other studies on magnetite particles synthesized by a chemical route that also have reported negligible strain for $16 \mathrm{~nm}$ magnetite particles (Mürbe et al., 2008). 0.3\% for 15 $\mathrm{nm}$ magnetite particles (Rudolph et al., 2010). 0.02\% for $31 \mathrm{~nm}$ magnetite particles (Jafari et al., 2014). Other reports on smaller nanoparticles give a strain of $0.4 \%$ for $\sim 6 \mathrm{~nm}$ particles (Chaki et al., 2015). Similarly, magnetite nanoparticles $\sim 7 \mathrm{~nm}$ wide prepared by a sol-gel process have been reported to experience a strain of $1.2 \%$ (Sciancalepore et al., 2015). The Williamson Hall plot for the reference sample also shows no strain from the Williamson Hall plot (Figure 5.2.2b, please see overleaf).

\subsubsection{Scanning electron microscopy and transmission electron microscopy}

SEM images of the magnetite nanoparticles made by a co-precipitation method show that have aggregates formed. Figure 5.2.3a shows a low magnification image that illustrates that there are large agglomerations that are $\sim 200 \mu \mathrm{m}$ wide. Figures 5.2.3b and 5.2.3c show aggregates of $\sim 1.5 \mu \mathrm{m}$ size. These are composed of smaller grains that are in the range of nanometres. There are large clumps with aggregates on the surface. In Figure 5.2.3d, it can be seen that 
there is a $15 \mu \mathrm{m}$ wide cluster that is composed of many small ones. The shapes are irregular and random.
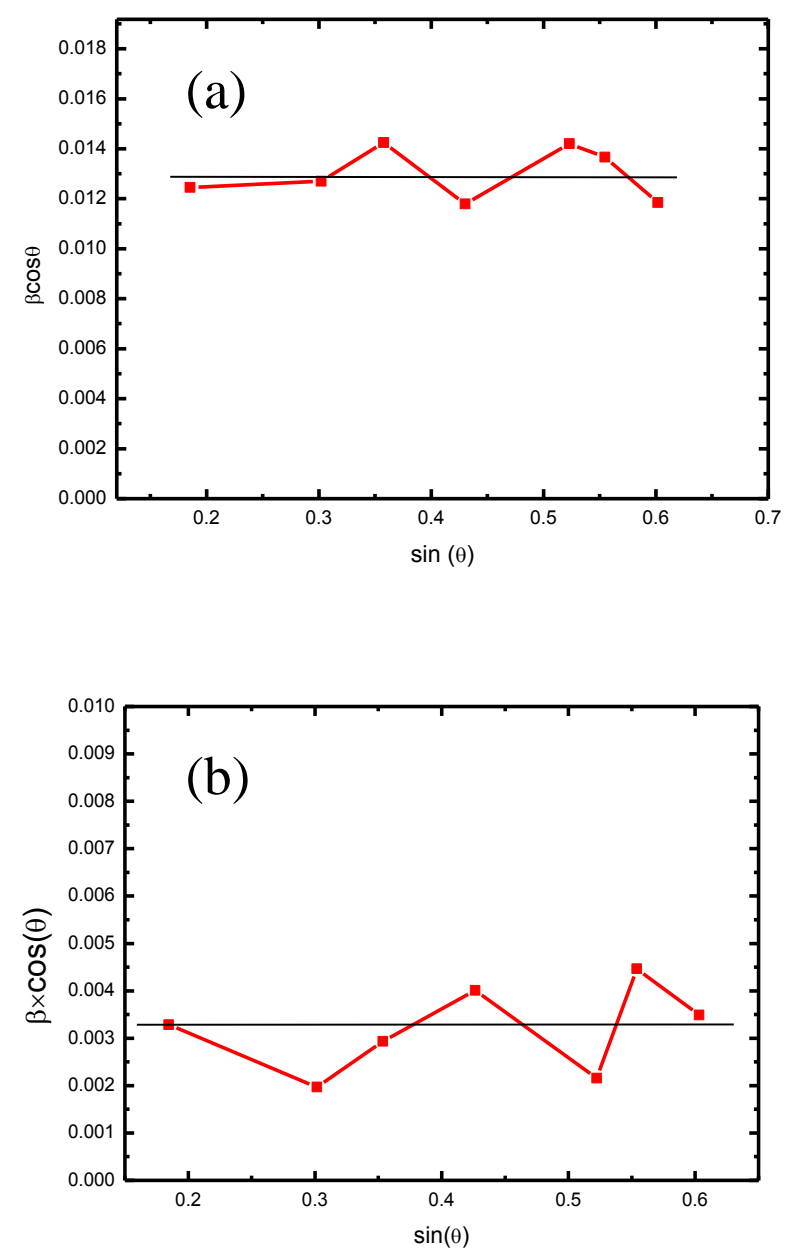

Figure 5.2.2. Williamson-Hall plot for the XRD data of the (a) synthesized iron oxide and (b) the magnetite reference sample. The straight lines are a guide to the eye. 


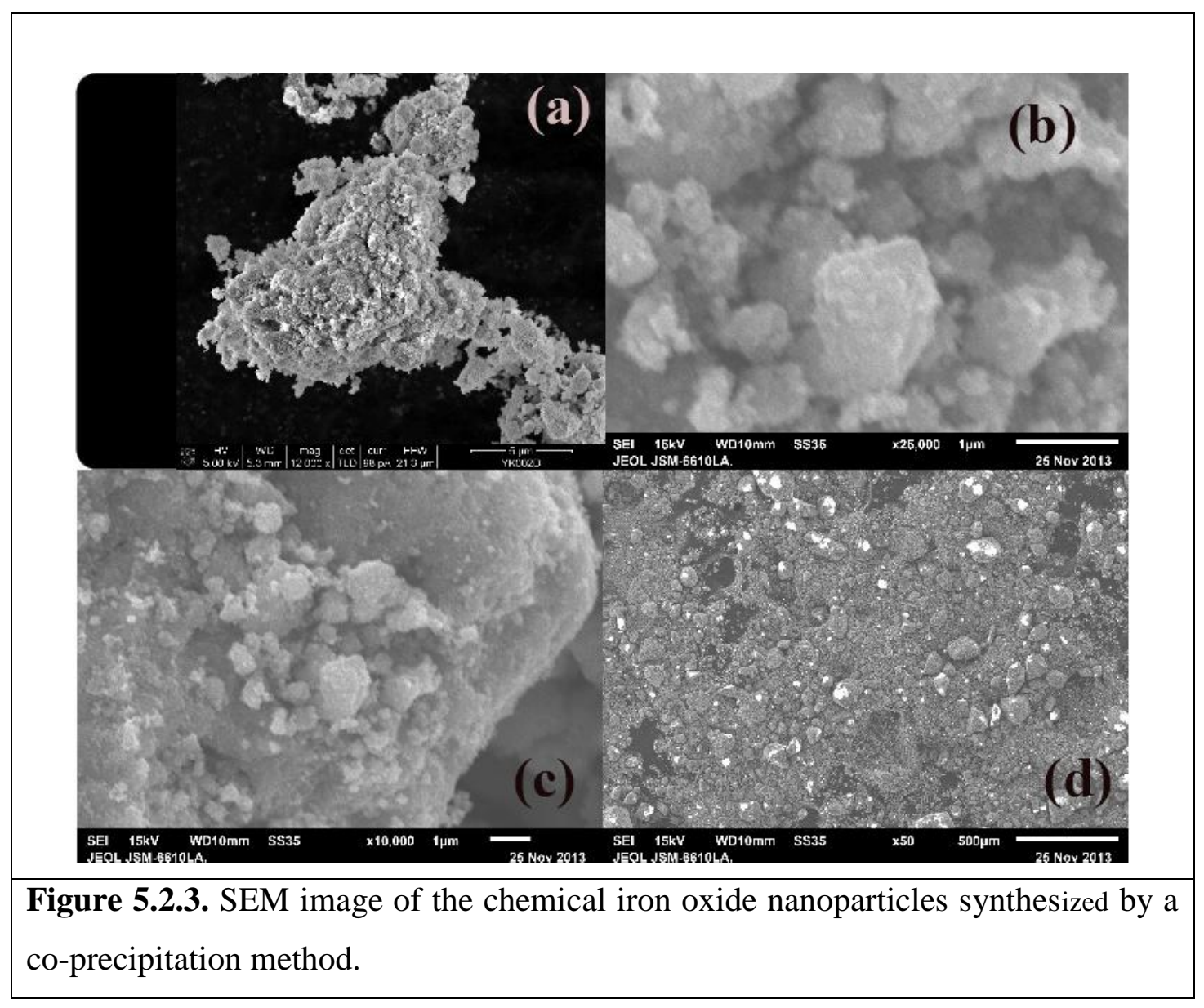

From TEM images in Figure 5.2.4, we see that Figure 5.2.4 (top) shows nanoparticles are present in the powder. These are largely spherical particles and these tend to agglomerate and form clusters. The small spherical particles are in contrast to the arc-discharge particles that are faceted and large as seen in section 5.2.1. Figure 5.2.4 (top) shows some of the clusters from the dispersion. Most of the particles are greater than $12 \mathrm{~nm}$, which is greater than the average crystallite size. This is possibly attributed to the presence of aggregates and also the presence of amorphous material that is surrounding cores that are crystalline. Within these particles loosely dispersed in the TEM grid, most of the aggregates are $\sim 50 \mathrm{~nm}$, as shown in Figure 5.2.4 (lower right). A large cluster is $300 \mathrm{~nm}$ long. There are some isolated nanoparticles as well. The nanoparticles present are largely uniform 
in size and these aggregate and form cross links due to the formation mechanism as well as the magnetic interactions.

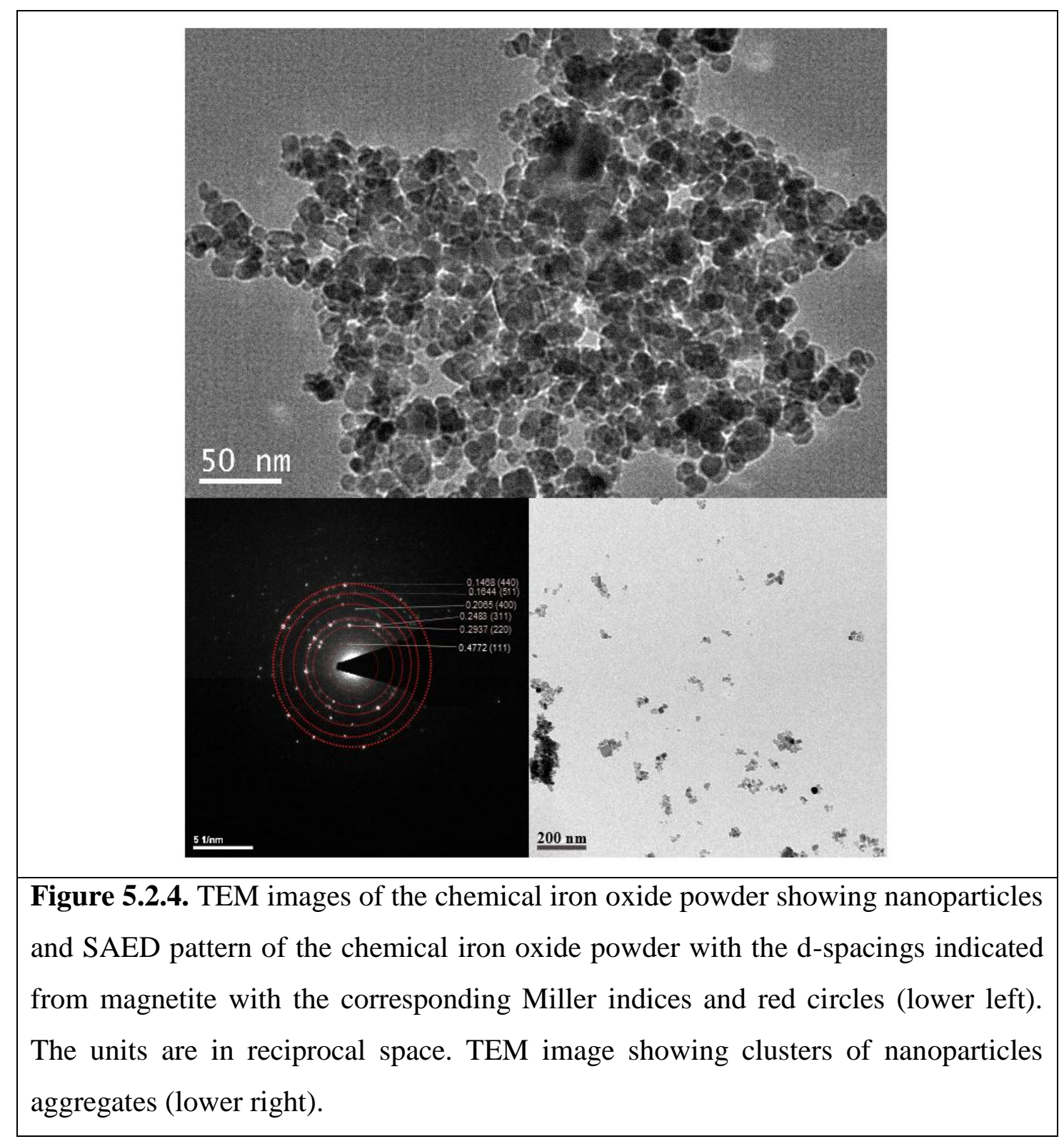

The SAED pattern from a cluster of particles is shown in Figure 5.2.4 (lower left). The sample is clearly polycrystalline with some large crystals indicated by the diffuse rings and the presence of dots on the pattern (Garnett et 
al., 2012). The dots are representative of the planes of the crystal and the grains are randomly oriented, leading to spotty rings. The spots forming the different rings are indicated by circles in the figure along with the d-spacings and Miller indices from magnetite. As-mentioned earlier, the d-spacings for magnetite are very close to those from maghemite and hence maghemite could also fit the SAED. We show below from the analysis of the Raman data that there is magnetite with some maghemite.

\subsubsection{Raman spectroscopy analysis}

Raman spectra performed using the $514.5 \mathrm{~nm}$ laser line from reference powders of magnetite, hematite and maghemite are shown in Figure 5.2.5. A large Rayleigh scattering background is seen, especially at the lower wavenumbers. Magnetite reference powder has a prominent peak at $667 \mathrm{~cm}^{-1}\left(\mathrm{~A}_{1 \mathrm{~g}}\right)$ with a width of $57 \mathrm{~cm}^{-1}$. The other peaks are at $547 \mathrm{~cm}^{-1}\left(\mathrm{~T}_{2 \mathrm{~g}}\right)$ and $363 \mathrm{~cm}^{-1}\left(\mathrm{~T}_{2 \mathrm{~g}}\right)$. There is no indication of a shoulder peak of maghemite at $700 \mathrm{~cm}^{-1}$. The peak fits to the Gauss at $667 \mathrm{~cm}^{-1}$.

The maghemite reference has a prominent peak at $700 \mathrm{~cm}^{-1}\left(\mathrm{~A}_{1 \mathrm{~g}}\right)$. The peak width is $101 \mathrm{~cm}^{-1}$. The other peaks are at $345 \mathrm{~cm}^{-1}$ and $530 \mathrm{~cm}^{-1}$ from the $\mathrm{T}_{2 \mathrm{~g}}$ and $\mathrm{E}_{\mathrm{g}}$ modes. The peak at $\sim 1400 \mathrm{~cm}^{-1}$ is possibly a second order phonon.

Hematite is indicated by the peaks at $299 \mathrm{~cm}^{-1}\left(\mathrm{E}_{\mathrm{g}}\right)$ and $425 \mathrm{~cm}^{-1}\left(\mathrm{E}_{\mathrm{g}}\right)$ and also a low intensity peak at $257 \mathrm{~cm}^{-1}\left(\mathrm{E}_{\mathrm{g}}\right)$. Hematite has a prominent peak at $1324 \mathrm{~cm}^{-1}$ that could be from $2^{\text {nd }}$ order phonon (Massey et al., 1990). Hematite also has a 2 magnon peak at this wavenumber that is more prominent at low temperatures. The peak width is $60 \mathrm{~cm}^{-1}$. The other peaks are at $299 \mathrm{~cm}^{-1}\left(\mathrm{E}_{\mathrm{g}}\right)$ and $425 \mathrm{~cm}^{-1}\left(\mathrm{E}_{\mathrm{g}}\right)$. There is also a low intensity peak at $234 \mathrm{~cm}^{-1}\left(\mathrm{~A}_{1 \mathrm{~g}}\right)$. 
Raman spectra at $633 \mathrm{~nm}$ from the magnetite powder are shown in Figure 5.2.6a. Magnetite is clearly indicated from the prominent peak at $664 \mathrm{~cm}^{-1}\left(\mathrm{~A}_{1 \mathrm{~g}}\right)$. There is an indication of the presence of maghemite from the peak at $700 \mathrm{~cm}^{-1}$. The Raman spectra for the iron oxide powder using the $514.5 \mathrm{~nm}$ laser line is shown in Figure 5.2.6c. The position of the peaks are $677 \mathrm{~cm}^{-1}$ and $717 \mathrm{~cm}^{-1}$. The peak width is $103 \mathrm{~cm}^{-1}$, which is made of two peaks that have contributions from magnetite and maghemite. The Lorentzian fits to these peaks give widths of 52 $\mathrm{cm}^{-1}$ and $44 \mathrm{~cm}^{-1}$ for the maghemite and the magnetite peaks (as shown in the inset), respectively. The presence of maghemite is evident from the shoulder peak at $717 \mathrm{~cm}^{-1}$. The presence of the maghemite peak is more evident using the $514 \mathrm{~nm}$ laser because the band gap is closer to the laser line $(2.2 \mathrm{eV}$; (Hashimoto et al., 1996)) and there is a resonance enhancement effect, causing an enhancement of the maghemite peak. The broadening could also be partly attributed to finite size effects. However, the bulk magnetite has been reported to have linewidths of $\sim 30 \mathrm{~cm}^{-1}$ (Shebanova and Lazor, 2003) which is comparable to the values seen here.

Raman spectra at $633 \mathrm{~nm}$ from the magnetite powder are shown in Figure 5.2.6a. Magnetite is clearly indicated from the prominent peak at $664 \mathrm{~cm}^{-1}\left(\mathrm{~A}_{1 \mathrm{~g}}\right)$. There is an indication of the presence of maghemite from the peak at $700 \mathrm{~cm}^{-1}$. The Raman spectra for the iron oxide powder using the $514.5 \mathrm{~nm}$ laser line is shown in Figure 5.2.6c. The position of the peaks are $677 \mathrm{~cm}^{-1}$ and $717 \mathrm{~cm}^{-1}$. The peak width is $103 \mathrm{~cm}^{-1}$, which is made of two peaks that have contributions from magnetite and maghemite. The Lorentzian fits to these peaks give widths of 52 $\mathrm{cm}^{-1}$ and $44 \mathrm{~cm}^{-1}$ for the maghemite and the magnetite peaks (as shown in the inset), respectively. The presence of maghemite is evident from the shoulder peak at $717 \mathrm{~cm}^{-1}$. The presence of the maghemite peak is more evident using the $514 \mathrm{~nm}$ laser because the band gap is closer to the laser line $(2.2 \mathrm{eV}$; (Hashimoto et al., 1996)) and there is a resonance enhancement effect, causing an 
enhancement of the maghemite peak. The broadening could also be partly attributed to finite size effects. However, the bulk magnetite has been reported to have linewidths of $\sim 30 \mathrm{~cm}^{-1}$ (Shebanova and Lazor, 2003) which is comparable to the values seen here.

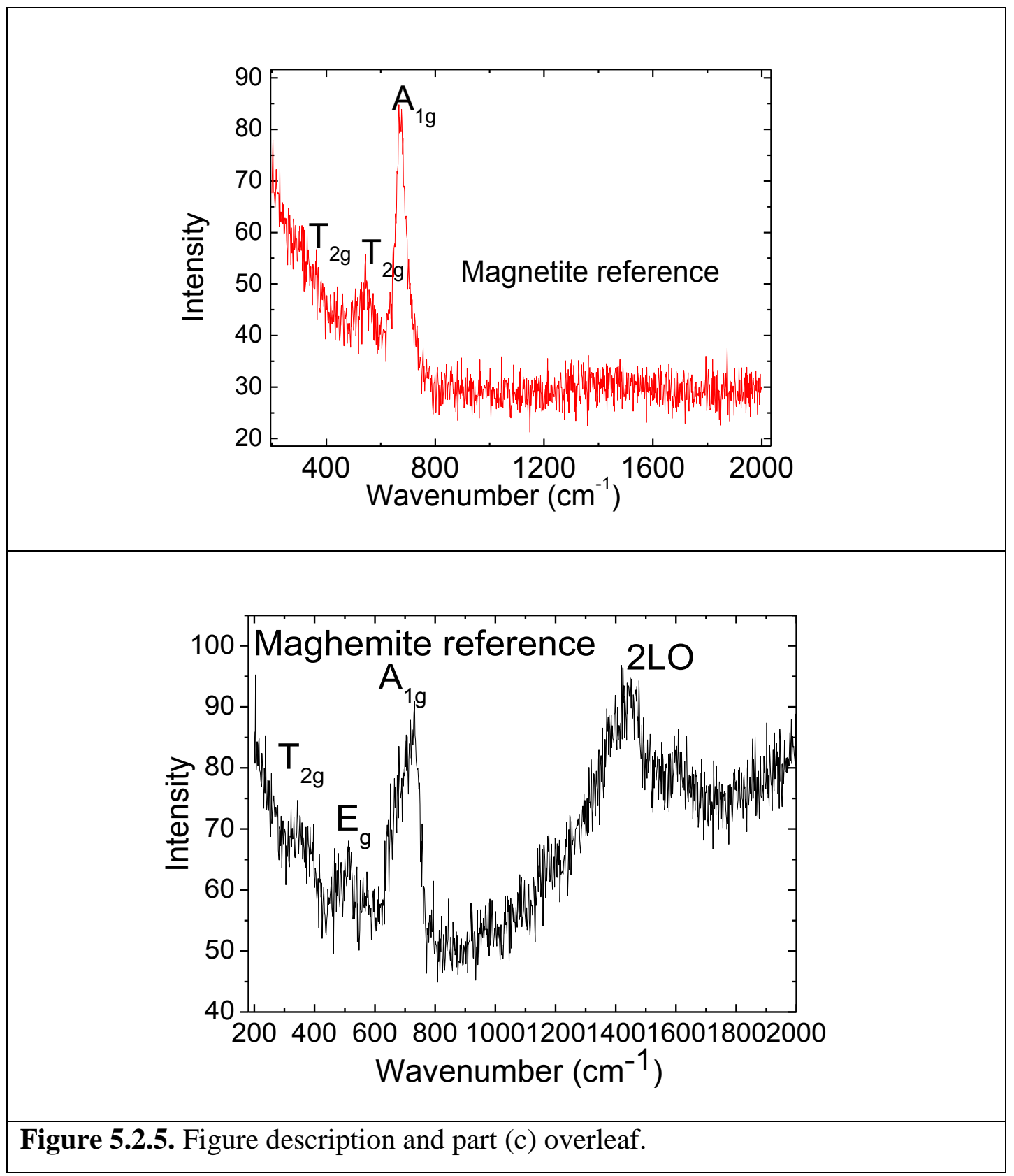




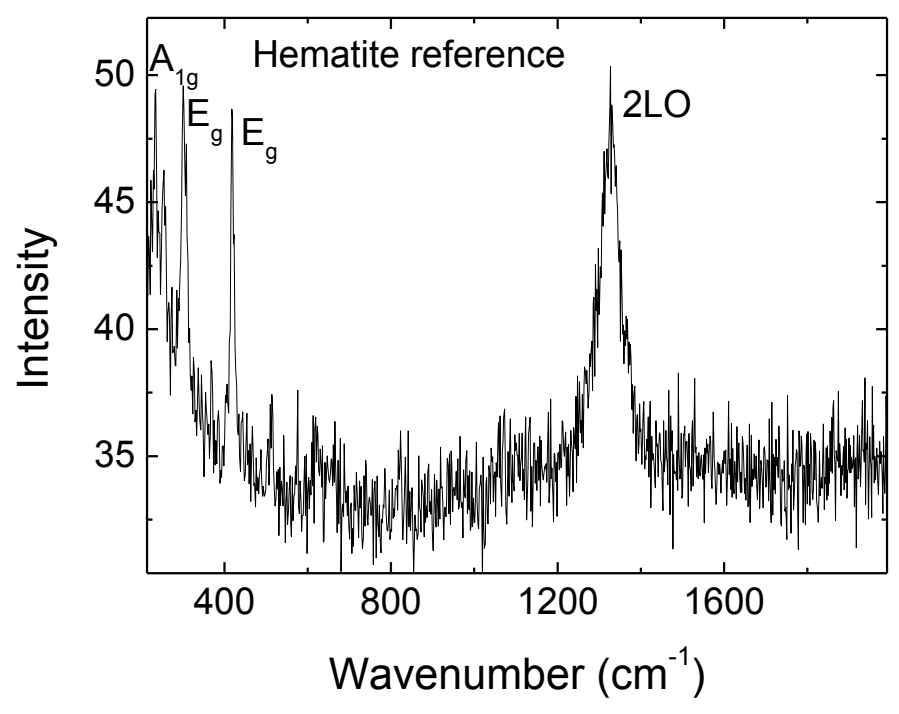

Figure 5.2.5. Raman spectra of reference powders of (a) magnetite, (b) maghemite and (c) hematite that were made by a chemical synthesis process.

Effect on broadening with increase in size was investigated by exposing the powder to a high intensity laser beam with a power of $90 \mathrm{~mW}$ for 7 minutes. The results from this experiment are shown in Figure 5.2.6b. There are prominent peaks (and modes) of $229 \mathrm{~cm}^{-1}\left(\mathrm{~A}_{1 \mathrm{~g}}\right), 247 \mathrm{~cm}^{-1}\left(\mathrm{E}_{\mathrm{g}}\right), 500 \mathrm{~cm}^{-1}\left(\mathrm{~A}_{1 \mathrm{~g}}\right), 620 \mathrm{~cm}^{-1}$ $\left(\mathrm{E}_{\mathrm{g}}\right)$ and $1350 \mathrm{~cm}^{-1}(2 \mathrm{LO})$ and these are of hematite. There was no evidence of magnetite peaks. This is a result of transition of phase to large particles of hematite. The exposure to high laser power results in the narrowing of the peak width of the magnetite, still found at $\sim 664 \mathrm{~cm}^{-1}$ by $36 \mathrm{~cm}^{-1}$. The peak at $1350 \mathrm{~cm}^{-1}$ is attributed to second order phonon scattering. The origin of the peak has been investigated to be from magnon scattering and is seen more prominently at low temperatures (Massey et al., 1990). This is an indication of agglomeration and clustering of the magnetite phase. The finite size effects result in the confinement of the phonon modes that result in narrow peak widths. There is also no indication of the peak at $700 \mathrm{~cm}^{-1}$, indicating the oxidation of maghemite. This is another 
reason for the narrowing of the width. Figure 5.2.6d shows the spectra from the annealed powder of magnetite. Here the peak is clearly only at $664 \mathrm{~cm}^{-1}$ and the full width half maximum of the peak is $39 \mathrm{~cm}^{-1}$, which is comparable to the magnetite peak width seen in Figure 5.2.6c. There is no evidence of a shoulder of maghemite.

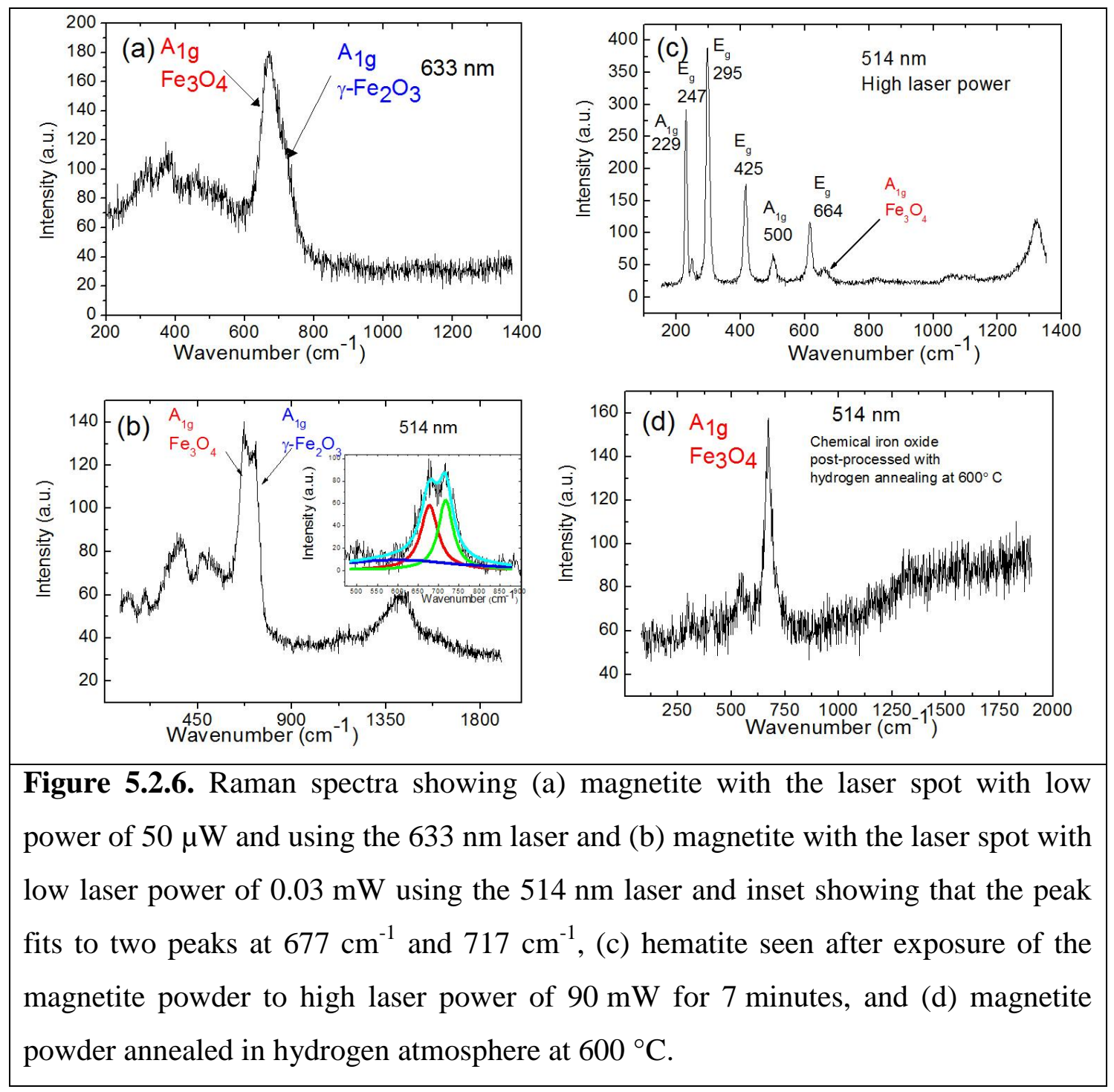




\subsubsection{Magnetization measurements}

Magnetization measurements at $300 \mathrm{~K}$ and $5 \mathrm{~K}$ were performed on the magnetite powder with a maximum applied field of $6 \mathrm{~T}$. The results are shown in Figure 5.2.7a. The sample is clearly ferromagnetic. The saturation moment is $\sim 78 \mathrm{emu} / \mathrm{g}$. It is noticeable that the moment does not show a saturation after $0.2 \mathrm{~T}$, but slowly increases up to $6 \mathrm{~T}$, and does not show any signs of saturation. It is possible that this is due to spin-glass effects. The coercivity of the sample at $300 \mathrm{~K}$ is $<10 \mathrm{mT}$, indicating that there could be a superparamagnetic contribution to the magnetization from some of the nanoparticles. The reported values of the saturation moment of magnetite nanoparticles is $98 \mathrm{emu} / \mathrm{g}$ at $0 \mathrm{~K}\left(\sim 3.2 \square_{\mathrm{B}}\right.$ per formula unit (Cullity and Graham, 2011)). The measured magnetization at $6 \mathrm{~T}$ is less than this, which is also seen in other small magnetite nanoparticles and can occur due to a spin-disordered shell.

Large particles are seen to dominate the magnetization in Fig 5.2.7b. The zero-field cooled field cooled (ZFC-FC) curves with the temperature range from $5 \mathrm{~K}$ to $300 \mathrm{~K}$ are divergent. There is irreversibility up to $300 \mathrm{~K}$. This indicates that any blocking temperature is higher than room temperature (Vitta, 2007). This is possibly due to the size of the nanoparticles where there average particle size estimated from the XRD data is $14 \mathrm{~nm}$, which is higher than the superparamagnetic limit. There is also a small transition at $\sim 100 \mathrm{~K}$. However, the magnitude of the change in magnetization is insignificant and it is possible that the Verwey transition is not seen here in the ZFC-FC data. 

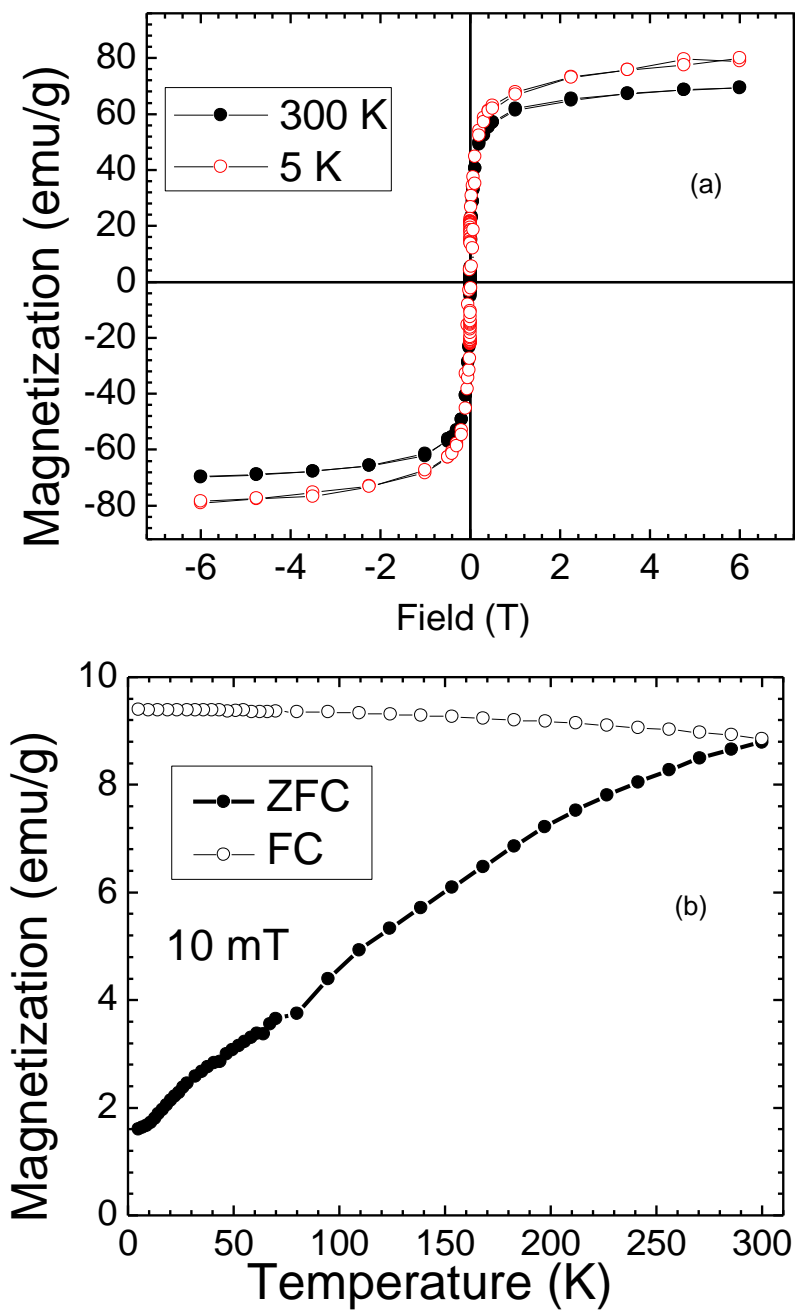

Figure 5.2.7. (a) Magnetization in $\mathrm{emu} / \mathrm{g}$ as a function of applied field of the chemical iron oxide powder at $300 \mathrm{~K}$ (closed circles) and $5 \mathrm{~K}$ (open circles). (b) Zero-field-cooled field-cooled curves of the iron oxide powder taken at $10 \mathrm{mT}$.

The saturation magnetization as a function of temperature, as shown in Figure 5.2.8a, indicates a decrease with increasing temperature due to spin waves. The data were fitted to equation 2.11, $M_{s}(T)=M_{s}(0)\left[1-B \times T^{\beta}\right]$, over the fill 
temperature range where $M_{\mathrm{s}}(0)$ is the magnetization saturation at $0 \mathrm{~K}$. A value of $\beta=2.05$ is obtained (not shown) but the fit is not good. The reason for this is that there are two temperature regions as seen in chapter 5.1 for magnetite made by the arc-discharge method. This can be seen more clearly in Figure 5.2.8b where $[\mathrm{m}(0)-\mathrm{m}(\mathrm{T})] / \mathrm{m}(0)$ is plotted. It is apparent that there is a departure below $\sim 110 \mathrm{~K}$ that likely to be due to the Verwey transition. This has not been reported before in small magnetite nanoparticles. There are very few studies but one study on $4 \mathrm{~nm}$ diameter magnetite nanoparticles found that the magnetization could be fitted to Equation 2.11 from low temperatures to $\beta=1.5$ up to room temperature without any evidence of a Verwey transition (Goya et al., 2003). A fit to Equation 2.11 was done in the low temperature region and it can be seen in Figure 5.2.8a that this provides a good fit to the data where $\beta=1.28$ and the $B=3.74 \mathrm{E}-5 \mathrm{~K}^{-1.28}$. $\beta$ is less that the 1.5 reported for $4 \mathrm{~nm}$ diameter magnetite nanoparticles (Goya et al., 2003) or for a magnetite single crystal (Aragón, 1992). The lower $\beta$ found by fitting the low temperature data in Figure 5.2.8a may be due to a spin-disordered region in the nanoparticle shells and spin wave propagation from the core into the shell region.

Low values of $\beta$ are known to occur in nanoparticles and it has been suggested that it can be less than 1.5 due to the effect of uncompensated surface spins (Zhang et al., 1998) that can result in temperature dependent spin-glass behaviour (Aquino et al., 2005). It is possible to obtain a good fit to the data using Equation 2.11 in the high temperature region as can be seen in Figure 5.2.8a. The fitted value of $\beta=1.96$ and $B=1.61 \mathrm{e}-6 \mathrm{~K}^{-1.96}$. The value of $\beta$ is slightly less than the $\beta$ $=2.26$ found for the arc-discharge powder. It is also larger than that reported for 4 nm diameter magnetite nanoparticles (Goya et al., 2003). 

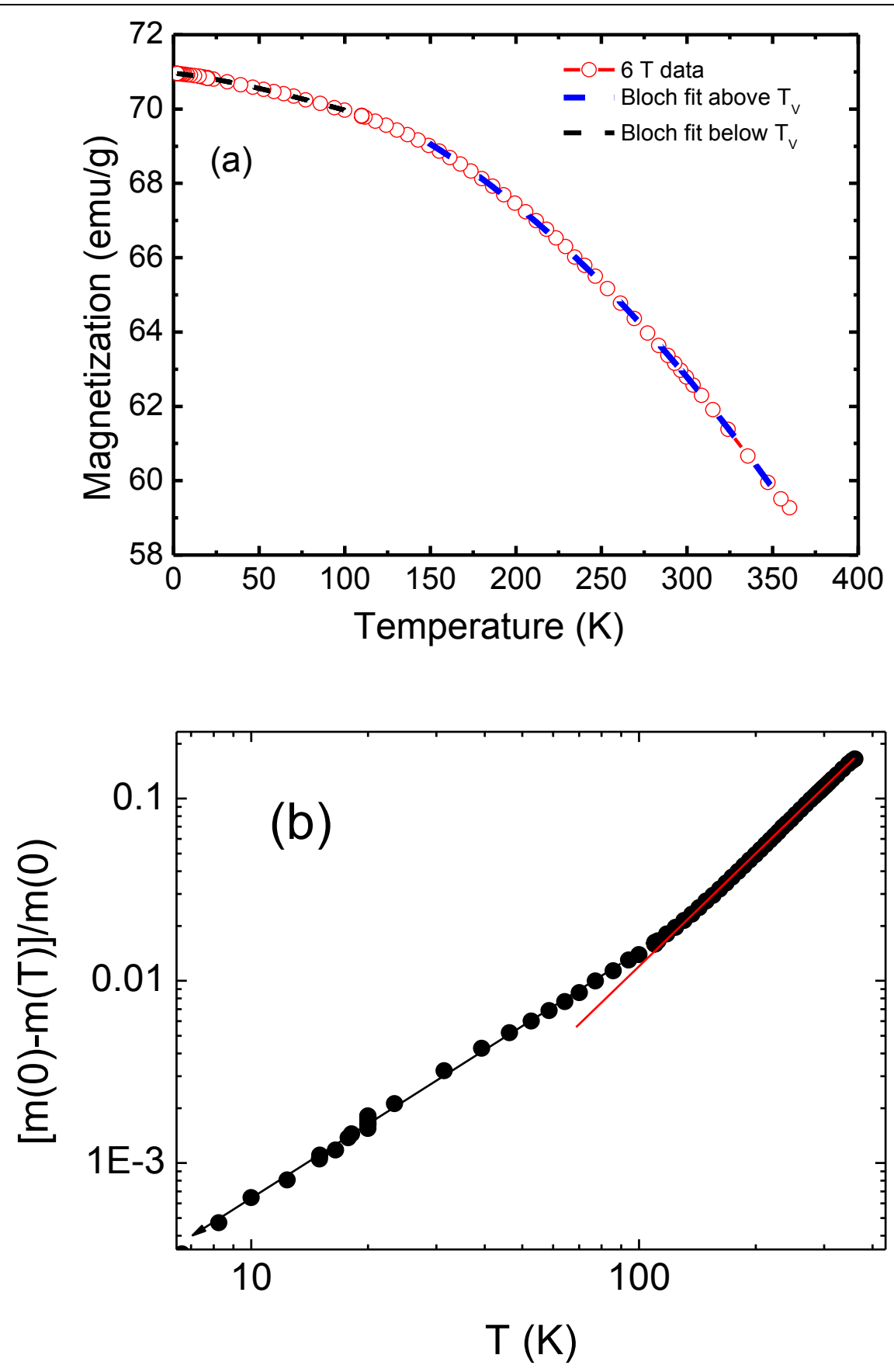

Figure 5.2.8. (a) Saturation magnetization against temperature with the fit to the Eq 2.11at low temperature and at higher temperature above the Verwey transition temperature. (b) Plot of $[\mathrm{m}(\mathrm{T})-\mathrm{m}(0)] / \mathrm{m}(0)$ against temperature. 


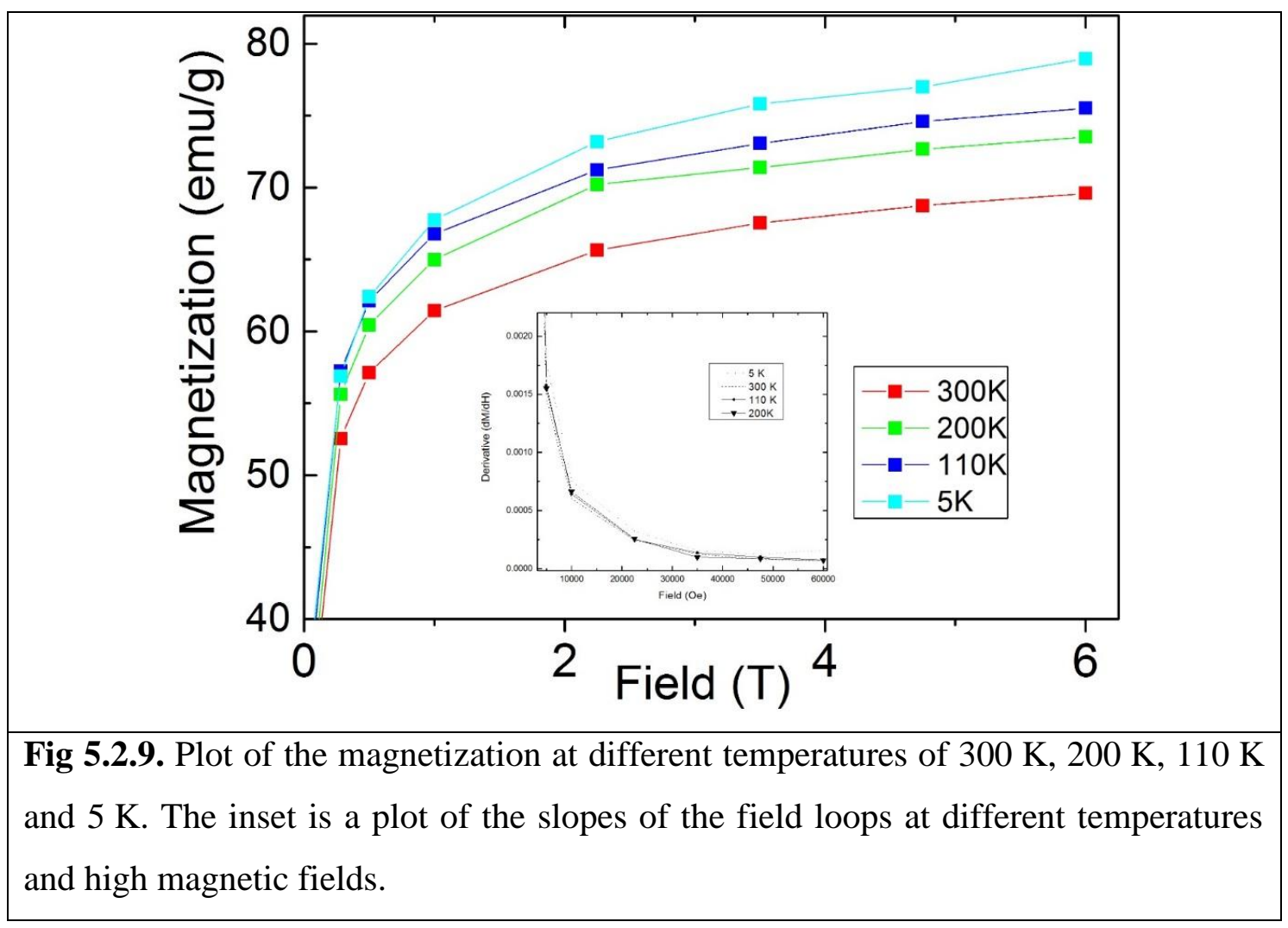

Spin-glass effects are evident from the non-saturation of the magnetization seen in Figure 5.2.9 and can arise from uncompensated surface spins. This most likely arises from the shell, where there are disordered spins and field-dependent magnetization measurements were performed to study this. The magnetization at different temperatures is an indication of the spin disorder increasing with decrease in the temperature. The plot of slopes of the field loops at different temperatures against the applied field is shown in the inset in Figure 5.2.9. This non-saturation seen at high fields is typical of spin glass behaviour (Binder and Young, 1986). 

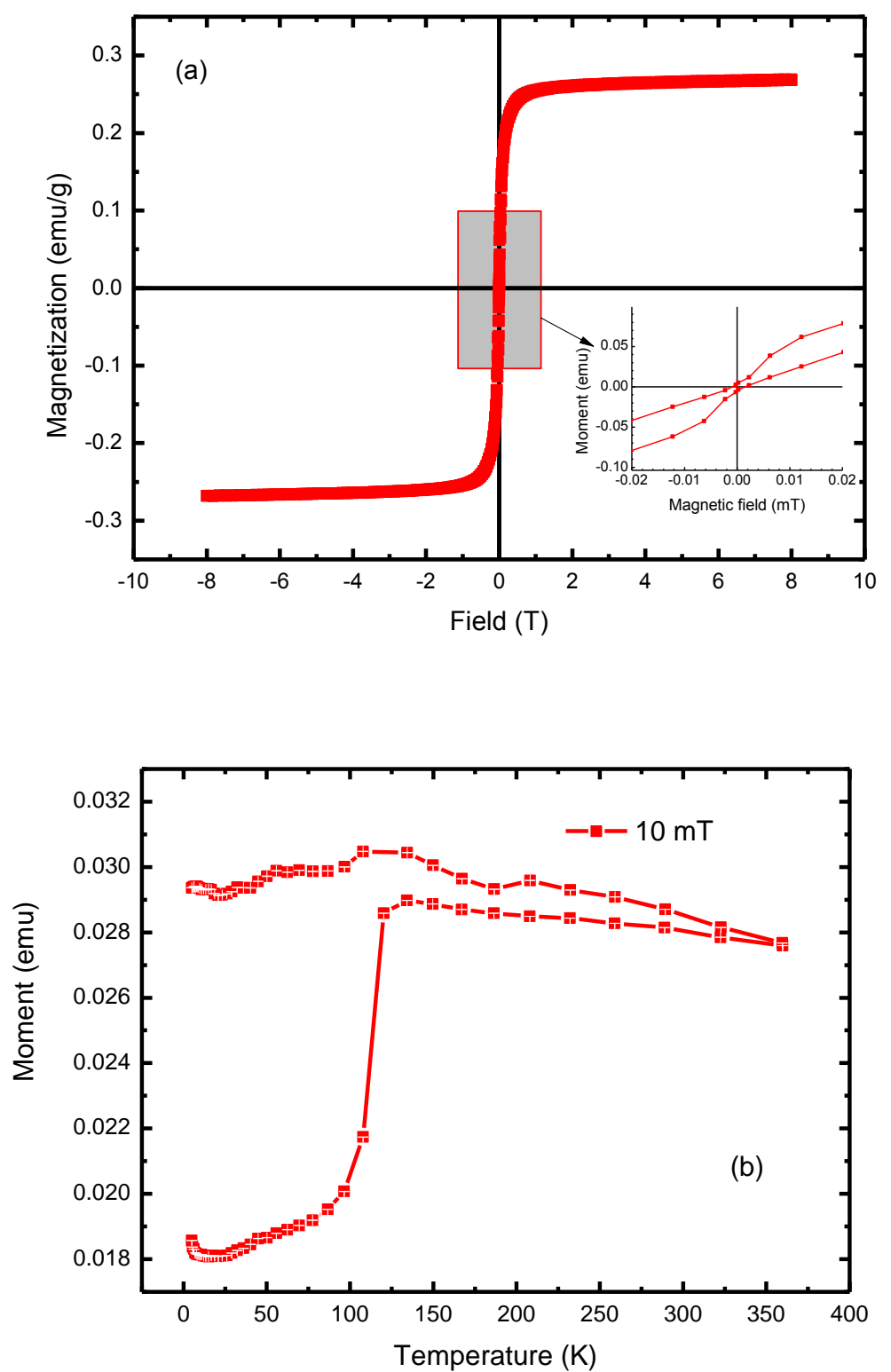

Figure 5.2.10. (a) Magnetization in emu/g as a function of applied field of the synthesized magnetite powder annealed at $600^{\circ} \mathrm{C}$ at $300 \mathrm{~K}$. Inset: Magnetization at $300 \mathrm{~K}$ with a maximum applied field of $0.15 \mathrm{~T}$ showing the hysteresis. (b) Zero-fieldcooled field-cooled curves of the annealed iron oxide powder taken at $10 \mathrm{mT}$. 
The extent of the rate of change with temperature is not significant. The slope of the magnetization plotted against the applied field is comparable for the temperatures from $5 \mathrm{~K}$ to $300 \mathrm{~K}$. This is indicative of thermal effects not being significant compared to the surface disorder. The increased spin disorder could be attributed to the smaller nanoparticles in this material. Dutta et al. (2009) reports a similar finding where $4 \mathrm{~nm}$ particles display a non-saturation of the magnetization and larger 6 to $12 \mathrm{~nm}$ particles display a saturation of the magnetization.

The magnetization measurements of the magnetite powder annealed for 1 hour at $600{ }^{\circ} \mathrm{C}$ in hydrogen is seen in Figure 5.2.10a. The magnetization is plotted against the field for measurements taken at $300 \mathrm{~K}$. The saturation moment corresponds to $90 \mathrm{emu} / \mathrm{g}$ and the coercivity is a bit lower than the powder before annealing at $<10 \mathrm{mT}$.The zero-field cooled field-cooled measurements at $10 \mathrm{mT}$ are shown in Figure 5.2.10b. The curves do not converge up to $360 \mathrm{~K}$ indicating that the large particles are a significant fraction of the powder, resulting in the lack of divergence. There is transition in the ZFC data at $125 \mathrm{~K}$ that is attributable to the Verwey transition, which is seen in larger magnetite particles that maybe octahedral and stoichiometric (Dewi et al., 2014) (Mitra et al., 2014) (Zhang et al., 2009). Thus, the higher saturation magnetization and the clear appearance of the Verwey transition in the ZFC data indicate that annealing has resulted in larger nanoparticles.

\subsubsection{Magnetoresistance measurements}

The magnetoresistance of the pressed pellet plotted against the field at $200 \mathrm{~K}$ and $300 \mathrm{~K}$ are shown in Figure 5.2.11. The magnetoresistance at $8 \mathrm{~T}$ reaches a value of $-8 \%$ at $200 \mathrm{~K}$ and $-6.2 \%$ at $300 \mathrm{~K}$. The value at $200 \mathrm{~K}$ is comparable to that seen in the arc discharge powder in chapter 5.1 and it is larger at room temperature. The temperature dependence of the magnetoresistance is 
also similar to that seen in Figure 5.2.7 and indicates that the magnetoresistance is also due to spin-dependent tunnelling. This shows that the semiconducting maghemite fraction is not high and below the percolation threshold. The magnetoresistance decreases with increasing temperature due to the degradation of spin polarization with increase in the temperature. Similar to the arc-discharge magnetite data it can be seen that the magnetoresistance data cannot be modelled using Equation 2.14. In particular, the magnetoresistance does not saturate at high fields, and this behaviour has been seen in granular systems and it is attributed to a spin disordered region at the grain boundaries. This leads to a phenomological $m_{\mathrm{gb}}$, given in equation 5.2 that was used to fit the data in conjunction with Equation 2.14. The resultant spin polarization at $300 \mathrm{~K}$ was $34 \%$ and the spin polarization at $200 \mathrm{~K}$ was $38 \%$. It is larger than that seen in the magnetite powders made by arc-discharge and it is less than the value expected for bulk magnetite (Dedkov et al., 2002).

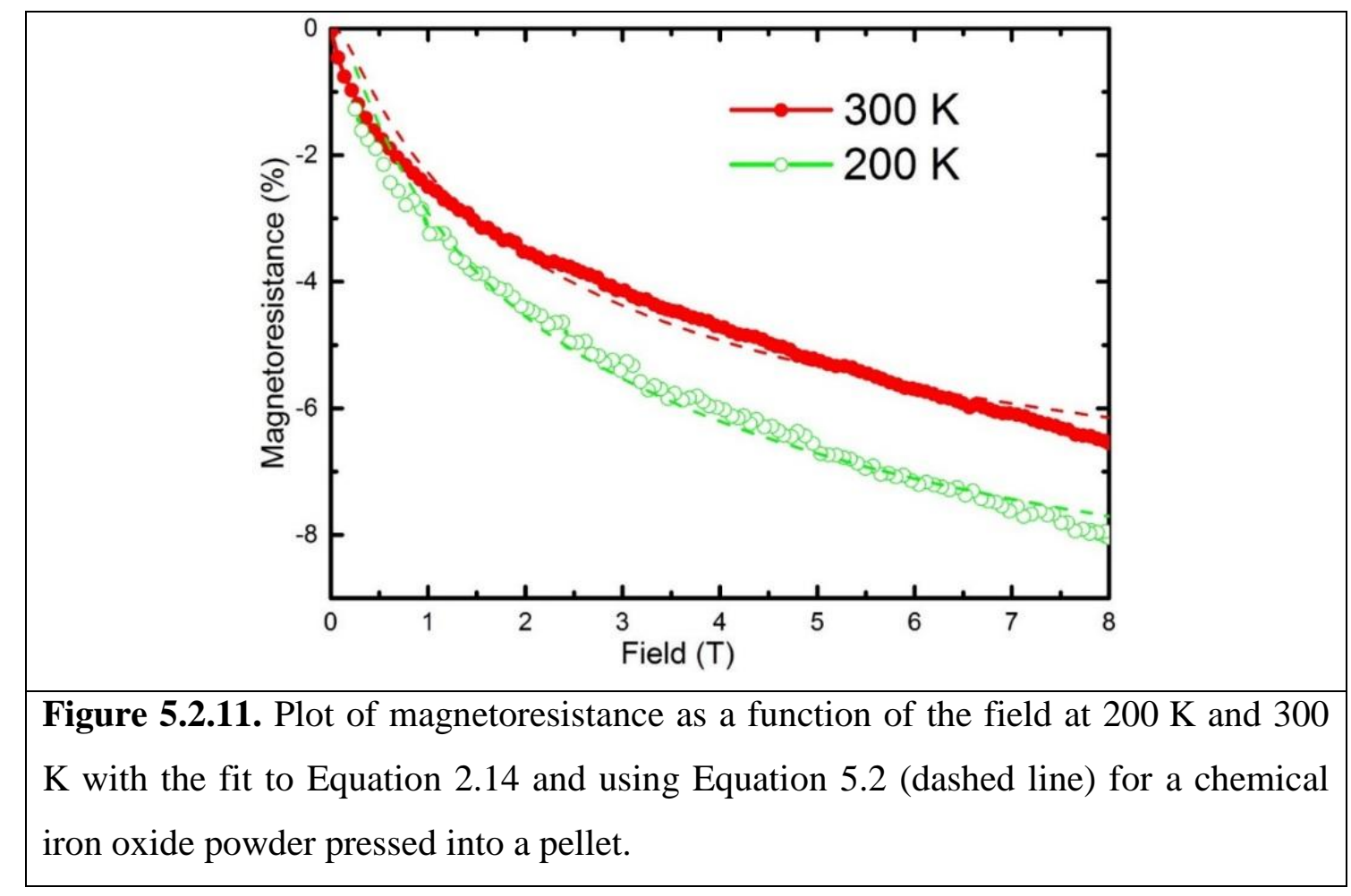


$\mathrm{R}(\mathrm{T})$ in the absence of a magnetic field is shown in Figure 5.2.12. The resulting plot of $\ln (\mathrm{R})$ against $\mathrm{T}^{-0.5}$ is shown in Figure 5.2.12.
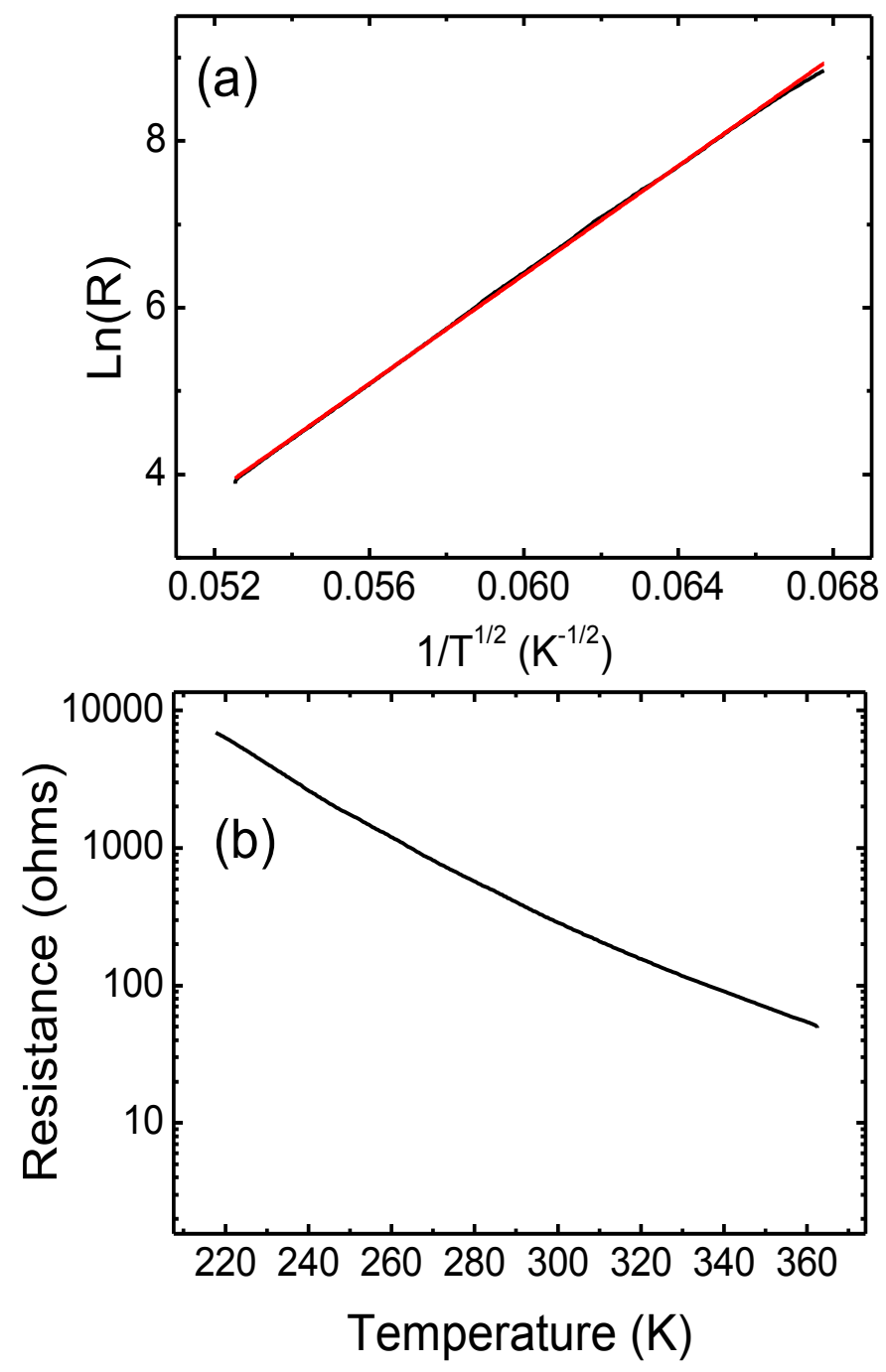

Figure 5.2.12. (a) Plot of $\operatorname{Ln}(\mathrm{R}(\mathrm{T})$ ) against $1 / \sqrt{T}$ and (b) plot of the resistance against temperature for the chemical iron oxide powder. 
The temperature dependence is similar to that seen in Chapter 5.1 and the presence of spin-dependent tunnelling indicates that it is not from variable range hoping and it is due to a Coulomb charging effect and tunnelling. A fit to Equation 5.4 gives $\kappa \mathrm{C}_{0} / \mathrm{k}_{\mathrm{B}}=25600 \mathrm{~K}$. This is larger than that seen in the iron oxide made by the arc discharge method in chapter 5.1, which is likely to be due to the smaller nanoparticle sizes that lead to a larger $\mathrm{E}_{\mathrm{c}}$ from $E_{c}=\left[e^{2} /\left[4 \pi \varepsilon_{0} d\right]\right] f(s / d)$. This results in a larger $\mathrm{C}_{0}$ because $C_{0}=2 \chi s_{0} E_{c}$. It is also possible that there is a difference in the tunnelling barrier height that leads to a larger $\kappa$.

\subsubsection{Conclusions}

The iron oxide powder made by a chemical co-precipitation method had an average crystallite size of $14 \mathrm{~nm}$ as estimated from the XRD measurements. The Raman spectra showed that there is magnetite and some maghemite. The moment per ion of the powder was less than that of the bulk magnetite, which has been reported in other magnetic nanoparticles. There was also a lack of saturation of the magnetization, possibly due to a spin-glass like effect, most likely an indication of the spin disordered layer at the surface. The Verwey transition was not apparent in the ZFC-FC data. However, it could be seen in the $M_{\mathrm{s}}(\mathrm{T})$ data at $\sim 110 \mathrm{~K}$. It was not observed in the $M_{\mathrm{s}}(\mathrm{T})$ data from a previous study on $4 \mathrm{~nm}$ diameter magnetite nanoparticles and shows that the Verwey transition disappears for magnetite diameters between $4 \mathrm{~nm}$ and $\sim 14 \mathrm{~nm}$. The Verwey transition is seen in the ZFC-FC data after annealing the powder at $600{ }^{\circ} \mathrm{C}$ in hydrogen, which is likely to be due to a large increase in the particle size. The exponent $\beta$ for the unannealed powder is less than that reported for bulk and $4 \mathrm{~nm}$ diameter nanoparticles, which may be due to a spin-disordered shell. Magnetoresistance is observed that is due to spin-dependent tunnelling. The resulting spin polarization from modelling the data in a phenomological model for the magnetite made by a chemical method was $\sim 38 \%$ at $8 \mathrm{~T}$. This is considerably higher than that obtained 
for the powders made by arc discharge. Further study could focus on using surfactants that could potantially bond with the nanoparticles surface and hence reduce the surface disorder. However, this approach is not expected to yield an improvement in the saturation magnetization values (Guardia et al., 2007). The temperature dependence of the resistance is due to a Coulomb charging effect and tunnelling. 


\subsection{Summary}

Iron oxide was made by an arc discharge synthesis method, with the powders containing magnetite with a small proportion of hematite phase. The powder contained nanoparticles as small as $7 \mathrm{~nm}$ as well as large particles with sizes up to $25 \mu \mathrm{m}$. Raman spectroscopy measurements also showed that there was some maghemite in the powders. The iron oxide powders made by coprecipitation method had an average crystallite size of $14 \mathrm{~nm}$. Raman spectroscopy measurements showed that there was magnetite with some maghemite.

The magnetization saturation of the arc-discharge powder is less than that observed for bulk magnetite, which is due to the presence of $\sim 26 \%$ of antiferromagnetic hematite. $\mathrm{M}_{\mathrm{s}}$ is also lower for iron oxide powder made by the co-precipitation method but in this case it is likely to be due to a spin-disordered nanoparticle shell. The Verwey transition is clearly seen in the ZFC-FC magnetization and $\mathrm{M}_{\mathrm{s}}(\mathrm{T})$ data for the arc-discharge powder, which is consistent with there being large magnetite nanoparticles. It is not seen in the ZFC-FC data for the $14 \mathrm{~nm}$ diameter powders, which has also been noted in other studies on magnetite nanoparticles. It can be seen in the $\mathrm{M}_{\mathrm{s}}(\mathrm{T})$ data and, by comparison with a previous study, that the Verwey transition disappears for nanoparticles sizes between $4 \mathrm{~nm}$ and $14 \mathrm{~nm}$.

There have been very few studies of $M_{s}(T)$ on magnetite reported in the literature. It was found in this study that the $\mathrm{M}_{\mathrm{s}}(\mathrm{T})$ exponent for the arc-discharge powder was 1.5 at low temperatures and the same as that reported for a single crystal. It was found that $J_{a b} / k_{\mathrm{B}}=42 \mathrm{~K}$. Above the Verwey transition is was $\beta=2.26$. The $14 \mathrm{~nm}$ diameter powder had $\beta=1.28$. This is less than that the expected value due to a spin-disordered shell. The exponent above the Verwey 
transition was $\beta=1.96$, which is slightly less than that found in the arc-discharge powder and close to that found in a previous study on $4 \mathrm{~nm}$ diameter magnetite nanoparticles.

The arc-discharge powder and the power containing $14 \mathrm{~nm}$ magnetite nanoparticles had a magnetoresistance that could be attributed to spin-dependent tunnelling between magnetite nanoparticles when pressed into pellets. In both cases the maximum magnetoresistance was large and magnetic field dependence of the magnetoresistance that could be modelled in terms of a spin-disordered region that resulted in a magnetoresistance that did not saturate at the highest measured magnetic field.

The electrical resistance for both powders in pressed pellet form had an exponential dependence on temperature with a temperature exponent of $1 / 2$. This can be attributed to a Coulomb charging effect and tunnelling between magnetite nanoparticles. In the case of the arc-discharge powders, the presence of a significant fraction of large particles means that the resistance is likely to be dominated by tunnelling between small nanoparticles that coat the larger particles. $\kappa \mathrm{C}_{0} / \mathrm{k}_{\mathrm{B}}=25600 \mathrm{~K}$ for the $14 \mathrm{~nm}$ magnetite nanoparticles. It is larger than that seen in two other studies on magnetite nanoparticles, which may be due to a smaller nanoparticles size or increased barrier height. It is also larger than the $\kappa \mathrm{C}_{0} / \mathrm{k}_{\mathrm{B}}=9700 \mathrm{~K}$ found for the arc-discharge powder and indicates that the average nanoparticles size seen in the percolation pathway is higher in the arc-discharge powder. 


\section{References}

Aragón, R., 1992. Magnetization and exchange in nonstoichiometric magnetite. Physical Review B 46, 5328.

Azaroff, L. V., Buerger, M. J., 1958. Powder method in X-ray crystallography.

Barakat, N.A.M., 2012. Synthesis and characterization of maghemite iron oxide $\left(\nu-\mathrm{Fe}_{2} \mathrm{O}_{3}\right)$ nanofibers: novel semiconductor with magnetic feature. Journal of Materials Science 47, 6237-6245. doi:10.1007/s10853-012-6543-7

Binder, K., Young, A.P., 1986. Spin glasses: Experimental facts, theoretical concepts, and open questions. Reviews in Modern Physics 58, 801-976. doi:10.1103/RevModPhys.58.801

Cai, W., Wan, J., 2007. Facile synthesis of superparamagnetic magnetite nanoparticles in liquid polyols. Journal of Colloid and Interface Science 305, 366-370. doi:10.1016/j.jcis.2006.10.023

Cerri, A., Mauri, D., Landolt, M., 1983. Surface magnetization and hydrogen chemisorption on Gd: A spin-polarized photoemission study. Physical Review B 27, 6526.

Chaki, S.H., Malek, T.J., Chaudhary, M.D., Tailor, J.P., Deshpande, M.P., 2015. Magnetite $\mathrm{Fe}_{3} \mathrm{O}_{4}$ nanoparticles synthesis by wet chemical reduction and their characterization. Advances in Natural Sciences: Nanoscience and Nanotechnology 6, 035009. doi:10.1088/2043-6262/6/3/035009

Chamritski, I., Burns, G., 2005. Infrared- and Raman-active phonons of magnetite, maghemite, and hematite: a computer simulation and spectroscopic study. The Journal of Physical Chemistry B 109, 4965-4968. doi:10.1021/jp048748h

Chernyshova, I.V., Hochella Jr, M.F., Madden, A.S., 2007. Size-dependent structural transformations of hematite nanoparticles. 1. Phase transition. Physical Chemistry Chemical Physics 9, 1736. doi:10.1039/b618790k

Chin, C.-J., Yiacoumi, S., Tsouris, C., 2002. Agglomeration of magnetic particles and breakup of magnetic chains in surfactant solutions. Colloids and Surfaces A: Physicochemical and Engineering Aspects 204, 63-72.

Cullity, B.D., Graham, C.D., 2011. Introduction to magnetic materials. John Wiley \& Sons.

Daou, T.J., Pourroy, G., Bégin-Colin, S., Grenèche, J.M., Ulhaq-Bouillet, C., Legaré, P., Bernhardt, P., Leuvrey, C., Rogez, G., 2006. Hydrothermal synthesis of monodisperse magnetite nanoparticles. Chemistry of Materials 18, 4399-4404. doi:10.1021/cm060805r

de Boer, C.B., Dekkers, M.J., 1996. Grain-size dependence of the rock magnetic properties for a natural maghemite. Geophysical Research Letters 23, 28152818.

Dedkov, Y.S., Rüdiger, U., Güntherodt, G., 2002. Evidence for the half-metallic ferromagnetic state of $\mathrm{Fe}_{3} \mathrm{O}_{4}$ by spin-resolved photoelectron spectroscopy. Physical Review B 65, 064417. 
Dewi, M.R., Skinner, W.M., Nann, T., 2014. Synthesis and phase transfer of monodisperse iron oxide $\left(\mathrm{Fe}_{3} \mathrm{O}_{4}\right)$ nanocubes. Australian Journal of Chemistry 67, 663. doi:10.1071/CH13595

Dutta, P., Pal, S., Seehra, M.S., Shah, N., Huffman, G.P., 2009. Size dependence of magnetic parameters and surface disorder in magnetite nanoparticles. Journal of Applied Physics 105, 07B501. doi:10.1063/1.3055272

Efros, A.L., Shklovskii, B.I., 1975. Coulomb gap and low temperature conductivity of disordered systems. Journal of Physics C: Solid State Physics 8, L49.

Fang, F., Futter, J., Markwitz, A., Kennedy, J., 2009. UV and humidity sensing properties of $\mathrm{ZnO}$ nanorods prepared by the arc discharge method. Nanotechnology 20, 245502. doi:10.1088/0957-4484/20/24/245502

Fang, M., Ström, V., Olsson, R.T., Belova, L., Rao, K.V., 2012. Particle size and magnetic properties dependence on growth temperature for rapid mixed co-precipitated magnetite nanoparticles. Nanotechnology 23, 145601. doi:10.1088/0957$4484 / 23 / 14 / 145601$

Garcia, V., Bibes, M., Barthélémy, A., Bowen, M., Jacquet, E., Contour, J.-P., Fert, A., 2004. Temperature dependence of the interfacial spin polarization of $\mathrm{La}_{2 / 3} \mathrm{Sr}$ 1/3 $\mathrm{MnO}_{3}$. Physical Review B 69, 052403. doi:10.1103/PhysRevB.69.052403

Garnett, E.C., Cai, W., Cha, J.J., Mahmood, F., Connor, S.T., Greyson Christoforo, M., Cui, Y., McGehee, M.D., Brongersma, M.L., 2012. Self-limited plasmonic welding of silver nanowire junctions. Nature Materials 11, 241-249. doi:10.1038/nmat3238

Gass, J., Poddar, P., Almand, J., Srinath, S., Srikanth, H., 2006. Superparamagnetic Polymer nanocomposites with uniform $\mathrm{Fe}_{3} \mathrm{O}_{4}$ nanoparticle dispersions. Advanced Functional Materials 16, 71-75. doi:10.1002/adfm.200500335

Goya, G.F., Berquó, T.S., Fonseca, F.C., Morales, M.P., 2003. Static and dynamic magnetic properties of spherical magnetite nanoparticles. Journal of Applied Physics 94, 3520. doi:10.1063/1.1599959

Guardia, P., Batlle-Brugal, B., Roca, A.G., Iglesias, O., Morales, M.P., Serna, C.J., Labarta, A., Batlle, X., 2007. Surfactant effects in magnetite nanoparticles of controlled size. Journal of Magnetism and Magnetic Materials 316, e756-e759.

Hashimoto, T., Yamada, T., Yoko, T., 1996. Third-order nonlinear optical properties of sol-gel derived $\alpha-\mathrm{Fe}_{2} \mathrm{O}_{3}, \gamma-\mathrm{Fe}_{2} \mathrm{O}_{3}$, and $\mathrm{Fe}_{3} \mathrm{O}_{4}$ thin films. Journal of Applied Physics 80, 3184. doi:10.1063/1.363258

Inoue, J., Maekawa, S., 1996. Theory of tunneling magnetoresistance in granular magnetic films. Physical Review B 53, R11927.

Jafari, A., Shayesteh, S.F., Salouti, M., Boustani, K., 2014. Dependence of structural phase transition and lattice strain of $\mathrm{Fe}_{3} \mathrm{O}_{4}$ nanoparticles on calcination temperature. Indian Journal of Physics 89, 551-560. doi:10.1007/s12648-0140627-y

Jubb, A.M., Allen, H.C., 2010. Vibrational spectroscopic characterization of hematite, maghemite, and magnetite thin films produced by vapor deposition. ACS Applied Materials \& Interfaces 2, 2804-2812. doi:10.1021/am1004943 
Karaagac, O., Kockar, H., 2012. Effect of synthesis parameters on the properties of superparamagnetic iron oxide nanoparticles. Journal of Superconductivity and Novel Magnetism 25, 2777-2781. doi:10.1007/s10948-011-1264-8

Koch, C., Ovid'ko, I., Seal, S., Veprek, S., 2007. Structural nanocrystalline materials: fundamentals and applications. Cambridge University Press.

Kumar, P.A., Ray, S., Chakraverty, S., Sarma, D.D., 2014. Magnetoresistance and electroresistance effects in $\mathrm{Fe}_{3} \mathrm{O}_{4}$ nanoparticle system. Journal of Experimental Nanoscience 9, 391-397. doi:10.1080/17458080.2012.662657

Langford, J.I., Wilson, A.J.C., 1978. Scherrer after sixty years: a survey and some new results in the determination of crystallite size. Journal of Applied Crystallography $11,102-113$.

Lin, C.-R., Chiang, R.-K., Wang, J.-S., Sung, T.-W., 2006. Magnetic properties of monodisperse iron oxide nanoparticles. Journal of Applied Physics 99, 08 N710. doi:10.1063/1.2172891

Liu, H., Jiang, E.Y., Bai, H.L., Zheng, R.K., Zhang, X.X., 2003. Thickness dependence of magnetic and magneto-transport properties of polycrystalline $\mathrm{Fe}_{3} \mathrm{O}_{4}$ films prepared by reactive sputtering at room temperature. Journal of Physics D: Applied Physics 36, 2950.

Liu, K., Zhao, L., Klavins, P., Osterloh, F.E., Hiramatsu, H., 2003. Extrinsic magnetoresistance in magnetite nanoparticles. Journal of Applied Physics 93, 7951-7953. doi:10.1063/1.1556133

Lopez Maldonado, K.L., de la Presa, P., de la Rubia, M.A., Crespo, P., de Frutos, J., Hernando, A., Matutes Aquino, J.A., Elizalde Galindo, J.T., 2014. Effects of grain boundary width and crystallite size on conductivity and magnetic properties of magnetite nanoparticles. Journal of Nanoparticle Research 16. doi:10.1007/s11051-014-2482-3

Ma, J., Wang, L., Wu, Y., Dong, X., Ma, Q., Qiao, C., Zhang, Q., Zhang, J., 2014. Facile Synthesis of $\mathrm{Fe}_{3} \mathrm{O}_{4}$ nanoparticles with a high specific surface area. Materials Transactions 55, 1900.

Massey, M.J., Baier, U., Merlin, R., Weber, W.H., 1990. Effects of pressure and isotopic substitution on the Raman spectrum of $\alpha-\mathrm{Fe}_{2} \mathrm{O}_{3}$ : Identification of two-magnon scattering. Physical Review B 41, 7822-7827. doi:10.1103/PhysRevB.41.7822

Mitra, A., Mohapatra, J., Meena, S.S., Tomy, C.V., Aslam, M., 2014. Verwey transition in ultrasmall-sized octahedral $\mathrm{Fe}_{3} \mathrm{O}_{4}$ nanoparticles. The Journal of Physical Chemistry C 118, 19356-19362. doi:10.1021/jp501652e

Mürbe, J., Rechtenbach, A., Töpfer, J., 2008. Synthesis and physical characterization of magnetite nanoparticles for biomedical applications. Materials Chemistry and Physics 110, 426-433. doi:10.1016/j.matchemphys.2008.02.037

Owens, F.J., Orosz, J., 2006. Effect of nanosizing on lattice and magnon modes of hematite. Solid State Communications 138, 95-98. doi:10.1016/j.ssc.2006.01.049 
Park, C., Peng, Y., Zhu, J.-G., Laughlin, D.E., White, R.M., 2005. Magnetoresistance of polycrystalline $\mathrm{Fe} 3 \mathrm{O} 4$ films prepared by reactive sputtering at room temperature. Journal of applied physics 97, 10 C303.

Prakash, T., Williams, G.V.M., Kennedy, J., Murmu, P.P., Leveneur, J., Chong, S.V., Rubanov, S., 2014. Synthesis and structural, magnetic and magnetotransport properties of permalloy powders containing nanoparticles prepared by arc discharge. Journal of Alloys and Compounds 608, 153-157. doi:10.1016/j.jallcom.2014.04.111

Rudolph, M., Turan, C., Kirchberg, S., Ziegmann, G., Peuker, U.A., 2010. Processing and Characterization of Highly Filled Polymer Nanoparticle Composites for Micro Injection Molding Applications, in: Proceeding European Congress of Composite Materials14. Budapest, Ungarn.

Schwertmann, U., Cornell, R.M., 2008. Iron oxides in the laboratory. John Wiley \& Sons.

Sciancalepore, C., Bondioli, F., Manfredini, T., Gualtieri, A., 2015. Quantitative phase analysis and microstructure characterization of magnetite nanocrystals obtained by microwave assisted non-hydrolytic sol-gel synthesis. Materials Characterization 100, 88-97. doi:10.1016/j.matchar.2014.12.013

Senn, M.S., Wright, J.P., Attfield, J.P., 2011. Charge order and three-site distortions in the Verwey structure of magnetite. Nature 481, 173-176. doi:10.1038/nature10704

Serrate, D., De Teresa, J.M., Algarabel, P.A., Fernández-Pacheco, R., Galibert, J., Ibarra, M.R., 2005a. Grain-boundary magnetoresistance up to $42 \mathrm{~T}$ in cold-pressed Fe3O4 nanopowders. Journal of Applied Physics 97. doi:10.1063/1.1868877

Serrate, D., De Teresa, J.M., Algarabel, P.A., Ibarra, M.R., Galibert, J., 2005b. Intergrain magnetoresistance up to $50 \mathrm{~T}$ in the half-metallic $\left(\mathrm{Ba}_{0.8} \mathrm{Sr}_{0.2}\right)_{2} \mathrm{FeMoO}_{6}$ double perovskite: Spin-glass behavior of the grain boundary. Physical Review B 71. doi:10.1103/PhysRevB.71.104409

Serrate, D., Teresa, J.M.D., Ibarra, M.R., 2007. Double perovskites with ferromagnetism above room temperature. Journal of Physics: Condensed Matter 19, 023201. doi:10.1088/0953-8984/19/2/023201

Shang, C.H., Nowak, J., Jansen, R., Moodera, J.S., 1998. Temperature dependence of magnetoresistance and surface magnetization in ferromagnetic tunnel junctions. Physical Review B 58, R2917.

Shebanova, O.N., Lazor, P., 2003. Raman spectroscopic study of magnetite $\left(\mathrm{FeFe}_{2} \mathrm{O}_{4}\right)$ : a new assignment for the vibrational spectrum. Journal of Solid State Chemistry 174, 424-430. doi:10.1016/S0022-4596(03)00294-9

Sheng, P., Abeles, B., Arie, Y., 1973. Hopping conductivity in granular metals. Physical Review Letters 31, 44.

Slavov, L., Abrashev, M.V., Merodiiska, T., Gelev, C., Vandenberghe, R.E., MarkovaDeneva, I., Nedkov, I., 2010. Raman spectroscopy investigation of magnetite nanoparticles in ferrofluids. Journal of Magnetism and Magnetic Materials 322, 1904-1911. doi:10.1016/j.jmmm.2010.01.005 
Smit, J., Wijn, H.P.J., 1959. Ferrites: physical properties of ferrimagnetic oxides in relation to their technical applications. Wiley.

Song, P.Y., Wang, J.F., Chen, C.P., Deng, H., Li, Y.D., 2006. Enhanced low field magnetoresistance of $\mathrm{Fe}_{3} \mathrm{O}_{4}$ nanosphere compact. Journal of Applied Physics 100, 044314. doi:10.1063/1.2335386

Stokes, A.R., Wilson, A.J.C., 1944. The diffraction of $X$ rays by distorted crystal aggregates-I. Proceedings of the Physical Society 56, 174.

Sun, Y., Ma, M., Zhang, Y., Gu, N., 2004. Synthesis of nanometer-size maghemite particles from magnetite. Colloids and Surfaces A: Physicochemical and Engineering Aspects 245, 15-19. doi:10.1016/j.colsurfa.2004.05.009

Venkatesan, M., Nawka, S., Pillai, S.C., Coey, J.M.D., 2003. Enhanced magnetoresistance in nanocrystalline magnetite. Journal of Applied Physics 93, 8023. doi:10.1063/1.1555371

Vitta, S., 2007. Nonlinear spin wave magnetization of solution synthesized $\mathrm{Ni}$ nanoparticles. Journal of Applied Physics 101, 063901. doi:10.1063/1.2710437

Williamson, G., Hall, W., 1953. X-ray line broadening from filed aluminium and wolfram. Acta Matallurgica 1, 22-31.

Wu, J.-H., Ko, S.P., Liu, H.-L., Kim, S., Ju, J.-S., Kim, Y.K., 2007. Sub 5 nm magnetite nanoparticles: Synthesis, microstructure, and magnetic properties. Materials Letters 61, 3124-3129. doi:10.1016/j.matlet.2006.11.032

Yanase, A., Siratori, K., 1984. Band structure in the high temperature phase of $\mathrm{Fe}_{3} \mathrm{O}_{4}$. Journal of the Physical Society of Japan 53, 312-317.

Zhang, L., Wu, J., Liao, H., Hou, Y., Gao, S., 2009. Octahedral $\mathrm{Fe}_{3} \mathrm{O}_{4}$ nanoparticles and their assembled structures. Chemical Communications 4378. doi:10.1039/b906636e

Zhang, Z., Satpathy, S., 1991. Electron states, magnetism, and the Verwey transition in magnetite. Physical Review B 44, 13319.

Zhao, L., Duan, L., 2010. Uniform Fe3O4 octahedra with tunable edge length - synthesis by a facile polyol route and magnetic properties. European Journal of Inorganic Chemistry 2010, 5635-5639. doi:10.1002/ejic.201000580

Ziese, M., Blythe, H.J., 2000. Magnetoresistance of magnetite. Journal of Physics: Condensed Matter 12, 13-28. doi:10.1088/0953-8984/12/1/302 


\section{Chapter 6}

\section{Dual implantation of iron and nickel into silicon dioxide}

It has previously been shown that dual sequential ion implantation can be used to create alloy or intermetallic nanoclusters. For example it has been used to create $\mathrm{Cu}-\mathrm{Ni}$ alloys in silicate glasses (Zuhr et al., 1998) and $\mathrm{Ag}-\mathrm{Cd}$ nanocluster composites in silica (Anderson et al., 1997). Previous studies have reported the dual implantation of iron and nickel into silicon and annealing has resulted in the formation of silicides (Li et al., 1997) and also the formation of $\mathrm{NiSi}_{2}$ and $\mathrm{FeSi}_{2}$ with the increase in annealing temperature ( $\mathrm{Li}$ et al., 1998). This is similar to iron implantation studies where high doses of iron leads to the formation of silicide (Balakirev et al., 2014) (Lakshantha et al., 2015), the potential for silicide formation being favourable in the absence or lower concentration of oxygen (Leveneur et al., 2014a).

A range of magnetic nanoparticles have been implanted that include $\mathrm{Ni}$ (Amekura et al., 2004a) (Malik et al., 2012) (Sprouster et al., 2011) and Co nanoparticles (Malik et al., 2012). Previous studies on ion implantation of iron into silicon dioxide followed by electron beam annealing have resulted in the formation of surface superparamagnetic nanoclusters (J. Leveneur et al., 2011) (Kennedy et al., 2011). Dual implantation of Co and Pt has been reported that leads to an enhanced coercivity and magnetic remanence. This is due to the formation of alloy that has formed successfully upon implantation (Malik et al., 2012). However, the formation of alloy nanoparticles of permalloy that could potentially have a low anisotropy, low coercivity and high permeability has not been reported.

This chapter presents the results from a study of the magnetic and structural properties of clusters formed by dual implantation of iron and nickel with two different $\mathrm{Ni}$ concentrations and the effects of electron beam annealing. 
Specifically, implantation and annealing was done with two nickel to iron fluence ratios of 82:18 and 45:55 with the same Ni fluence. A higher Ni fluence was also studied with a nickel to iron ratio 50:50. The ratios of 82:18 and 45:55 have concentration profiles that are roughly comparable to the P78 and the P45 synthesized in Chapter 3. As discussed in Chapter 2, these ratios were chosen because these exhibit a low magnetocrystalline anisotropy and the highest magnetization saturation, respectively.

\subsection{Implantation of $\mathrm{Ni}_{0.82} \mathrm{Fe}_{0.18}$ in $\mathrm{SiO}_{2}$}

This section reports the results from the dual implantation of iron and nickel into $\mathrm{SiO}_{2}$ with a $\mathrm{Ni}$ to $\mathrm{Fe}$ fluence ratio of 82:18. As shown below, this results in a peak ratio of $\mathrm{Fe}_{0.82} \mathrm{Ni}_{0.18}$ that is close to the commonly used permalloy concentration ratio of 78:22. The structural properties and the magnetic properties were studied and the compared to previous iron and nickel implantation studies.

\subsubsection{Synthesis technique}

$\mathrm{Ni}_{x} \mathrm{Fe}_{1-x}$ nanoclusters embedded in silicon dioxide were made using the low-energy implanter and EBA systems mentioned in chapter 3 where the EBA was done at $1000{ }^{\circ} \mathrm{C}$ under high vacuum. The implantation was done into $500 \mathrm{~nm}$ $\mathrm{SiO}_{2}$ on $0.3 \mathrm{~mm}$ crystalline silicon substrates with an implantation energy of 10 $\mathrm{keV}$. The implantation of ${ }^{58} \mathrm{Ni}^{+}$was carried out first and then followed by ${ }^{56} \mathrm{Fe}^{+}$ with a low current of $<2 \mu \mathrm{A}$ and pressure of $10^{-7}$ mbar. The implanted nickel fluence was $2 \times 10^{16}$ at. $/ \mathrm{cm}^{2}$. ${ }^{56} \mathrm{Fe}^{+}$was implanted into the nickel-implanted samples with the fluence of $4.5 \times 10^{15}$ at. $/ \mathrm{cm}^{2}$. The nickel to iron fluence ratio was $82 \%$. EBA was used to anneal the sample at $1000{ }^{\circ} \mathrm{C}$ for a duration of 1 hour. The heating rate used was $5{ }^{\circ} \mathrm{C} / \mathrm{s}$. After holding at $1000{ }^{\circ} \mathrm{C}$ for 1 hour, the sample was slowly cooled at a rate of $3{ }^{\circ} \mathrm{C} / \mathrm{s}$ down to $300{ }^{\circ} \mathrm{C}$ and furnace-cooled down to room temperature. D-TRIM simulations (Biersack et al., 1991) were used for depth and concentration profiling. RBS measurements were performed using a $2 \mathrm{MeV} \mathrm{He}{ }^{+}$beam and current of $\sim 20 \mathrm{nA}$. The fluences were obtained by fitting 
using RUMP software. Cross-sectional TEM was performed on samples prepared using the focused ion beam (FIB) lift-out technique (Gianuzzi, 1998). To reduce charging, the samples were carbon coated ( 10 nm film thickness). For sample preparation, a 100-nm thick Pt protection layer was deposited using the electronbeam deposition facility of the FEI Nova Nanolab 200 dual-beam FIB system. FIB milling at $5 \mathrm{keV}$ was used at the final stage of sample preparation process. TEM study was conducted using FEI Tecnai TF20 electron microscope operated at $200 \mathrm{kV}$. Magnetization measurements were performed on a Quantum Design SQUID magnetometer.

\subsubsection{Structural measurements}

\subsubsection{D-TRIM simulations}

The results from D-TRIM simulations of the nickel and iron concentration profiles can be seen in Figure 6.1.1 after nickel implantation into silicon dioxide with a fluence of $2 \times 10^{16}$ at. $/ \mathrm{cm}^{2}$ that was followed by iron implantation with a fluence of $4.5 \times 10^{15}$ at. $/ \mathrm{cm}^{2}$. The Fe fraction was calculated from the values and the resultant ratios of the relative concentrations of iron to nickel with depth are also plotted in Figure 6.1.1. The simulated average estimated depth of the nickel in silicon dioxide is $\sim 15 \mathrm{~nm}$ and the maximum depth is $35 \mathrm{~nm}$. The average straggling depth is $\sim 20 \mathrm{~nm}$ and the depth of the iron implanted into this system with nickel implanted into silicon dioxide is similar, which implies that the implanted layer could be a mixture of the iron and nickel with a surface-rich $\mathrm{Ni}$ layer. Of the atoms with depth $>13 \mathrm{~nm}, 59 \%$ of these implanted atoms have an average nickel to iron ratio equivalent to $82: 18$. As the depth is reduced, the $\mathrm{Ni}$ fractional concentration goes from $82 \%$ to $100 \%$. Thus, the Fe fraction after implantation is expected to vary from 0 at the surface to $\sim 0.18$ further into the film. 


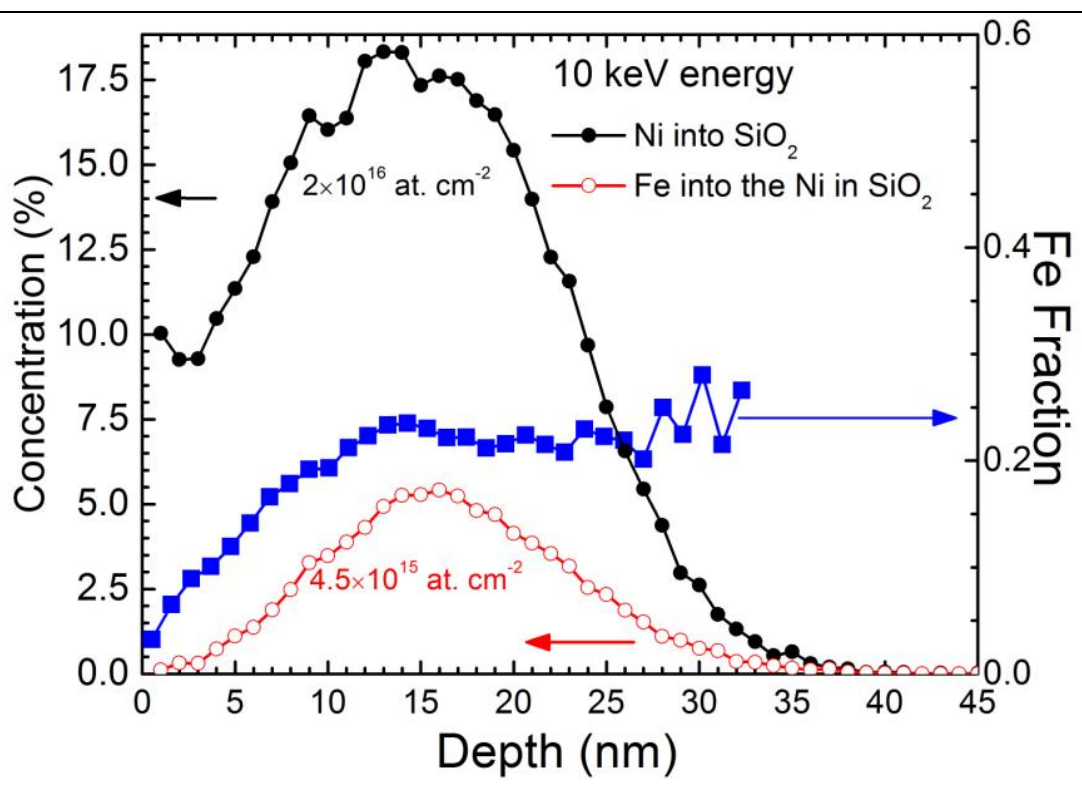

Figure 6.1.1. D-TRIM simulations for the implantation of Ni into silicon dioxide at a fluence of $2 \times 10^{16}$ at. $/ \mathrm{cm}^{2}$ (filled circles) and subsequent implantation of $\mathrm{Fe}$ with a fluence of $4.5 \times 10^{15}$ at. $/ \mathrm{cm}^{2}$ (open circles) into the Ni-implanted films at $10 \mathrm{keV}$ and the resultant $\mathrm{Fe}$ fraction as a function of depth (filled squares).

\subsubsection{Rutherford backscattering analysis}

Figure 6.1.2 shows the RBS data for the as-implanted film and the film annealed for 1 hour at $1000{ }^{\circ} \mathrm{C}$. The silicon edge, the silicon dioxide on silicon interface edge, and the oxygen edge can be clearly seen in the data. The peak at $\sim 1.5 \mathrm{MeV}$ is due to $\mathrm{Ni}$ and $\mathrm{Fe}$ in the film where the $\mathrm{Ni}$ and $\mathrm{Fe}$ edges that are indicated on the figure cannot be clearly resolved. After annealing, the Ni/Fe peak has significantly broadened and shifted to lower energy, which indicates that there has been diffusion into the $\mathrm{SiO}_{2}$ film. This was not observed after Fe implantation with a similar fluence and EBA at $1000{ }^{\circ} \mathrm{C}$ for 1 hour (J. Leveneur et al., 2011) (Kennedy et al., 2012b). There is also some $\mathrm{Ni} / \mathrm{Fe}$ at the $\mathrm{Si} / \mathrm{SiO}_{2}$ interface after annealing as can be seen by the small peak at $1.17 \mathrm{MeV}$ in the inset to Figure 6.1.2. Some $\mathrm{Fe}$ at the $\mathrm{Si} / \mathrm{SiO}_{2}$ interface was also seen after $\mathrm{Fe}$ implantation and EBA (Kennedy et al., 2012a) and hence it is not clear if it is $\mathrm{Fe}, \mathrm{Ni}$, or $\mathrm{Ni}_{x} \mathrm{Fe}_{1-x}$. 
EBA of Fe implanted films is known to sputter $\mathrm{Si}$ and oxygen and reduce the $\mathrm{SiO}_{2}$ layer thickness by $\sim 11 \mathrm{~nm} /$ hour (Kennedy et al., 2012b). This is likely to also occur for the $\mathrm{Ni}_{x} \mathrm{Fe}_{1-x}$ films after EBA but it cannot be resolved in Figure 6.1.2. The RBS data were fitted using RUMP software to obtain the total $\mathrm{Ni}$ and $\mathrm{Fe}$ fluences. They were $2.45 \times 10^{16}$ at. $/ \mathrm{cm}^{2}$ and $2.67 \times 10^{16}$ at. $/ \mathrm{cm}^{2}$ for the as-implanted film and after EBA, respectively, where they are the same as the implanted fluence within the experimental uncertainty of $\pm 4 \%$.

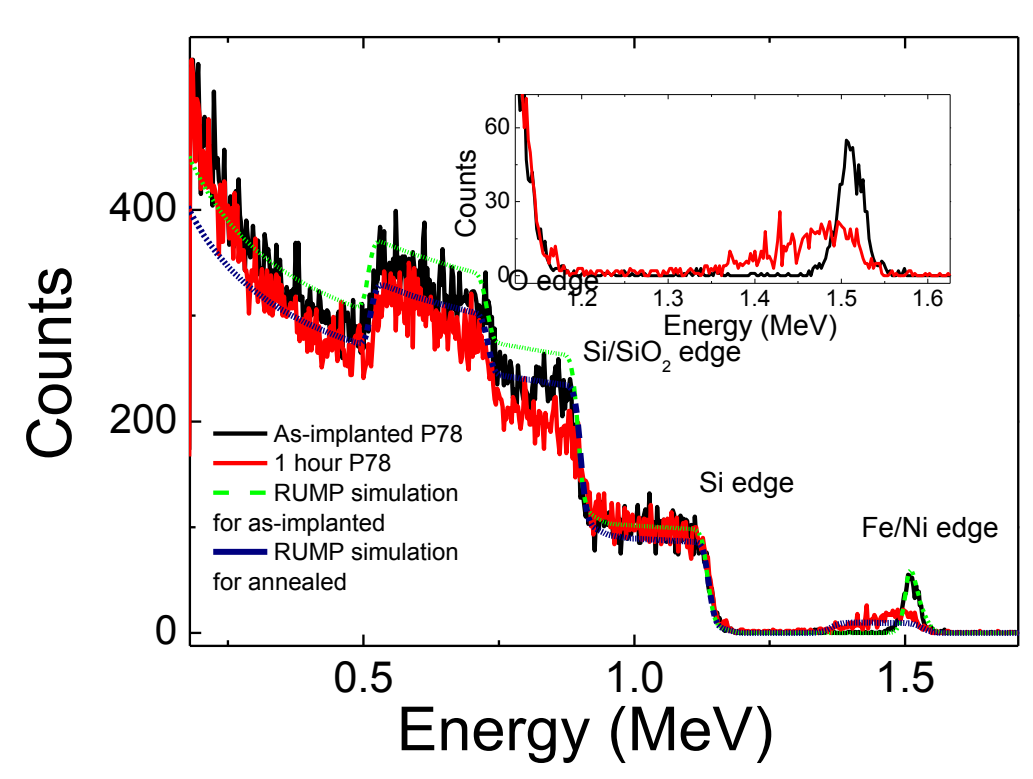

Figure 6.1.2. Rutherford backscattering data for the as-implanted sample (dashed curve) and the sample annealed for 1 hour at $1000{ }^{\circ} \mathrm{C}$ (solid curve) with RUMP simulations. Inset: expanded RBS plot.

\subsubsection{Transmission electron microscopy measurements}

TEM measurements were made of the as-implanted sample and the images can be seen in Figure 6.1.3a. The Pt, carbon, and $\mathrm{SiO}_{2}$ layers are indicated on the main image. There is also a $\mathrm{Ni}_{x} \mathrm{Fe}_{1-x}$ layer that can be seen clearer in the inset to Figure 6.1.3a. It has a width of $\sim 20 \mathrm{~nm}$, which is comparable to the straggling depth seen in the D-TRIM simulations. It is apparent in Figure 6.1.3a 
that the implanted region contains some small nanoparticles that are $\sim 4 \mathrm{~nm}$ wide. Nanoparticles are known to occur after ion implantation when the implanted ion concentration exceeds the solubility limit (Yuan et al., 2009). A previous study on $15 \mathrm{keV} \mathrm{Fe}$ implantation into $\mathrm{SiO}_{2}$ found that this solubility limit ranged from $2.3 \%$ to $6.7 \%$ (Jérôme Leveneur et al., 2011) where Fe nanoparticles were observed by TEM with diameters less than $5 \mathrm{~nm}$ for fluences of $10^{16} \mathrm{at} . / \mathrm{cm}^{2}$ and $\sim 20 \mathrm{~nm}$ for fluences of $10^{17}$ at. $/ \mathrm{cm}^{2}$ (Leveneur et al., 2014b). Ni nanoparticles have also been observed to form after implantation into $\mathrm{SiO}_{2}$ with a fluence of $3.8 \times 10^{16}$ at./ $\mathrm{cm}^{2}$ and an implantation energy of $60 \mathrm{keV}$ (Amekura et al., 2004b). From D-TRIM calculations, it is estimated that the corresponding peak concentration is $12 \%$, which is lower than our peak Ni concentration of $18 \%$. The resultant nanoparticles diameters from the $60 \mathrm{keV}$ study were less than $6 \mathrm{~nm}$ with an average value of $2.9 \mathrm{~nm}$ and hence comparable to the nanoparticle diameters found in our study.

Figure 6.1.3b shows TEM images after EBA for 1 hour where it can be seen that there is a bimodal particle size distribution where there are large nearly spherical nanoparticles near the surface and smaller nanoparticles that extend further into the $\mathrm{SiO}_{2}$ film. The larger particles have diameters that range from $40 \mathrm{~nm}$ to $75 \mathrm{~nm}$ and a $44 \mathrm{~nm}$ diameter nanoparticle can be seen in the right inset to Figure 6.1.3b. From these and other TEM images (not shown), it was found that particle size of the larger particles ranged from 35 to $110 \mathrm{~nm}$ with an average diameter of $56 \mathrm{~nm}$. Some sputtering of the $\mathrm{SiO}_{2}$ surface has occurred that leads to partial penetration of some large nanoparticles through the surface as can also be seen in the inset. There is also a nanoparticle that appears to be flattened close to the surface of the film that could be due to the growth mechanism. 


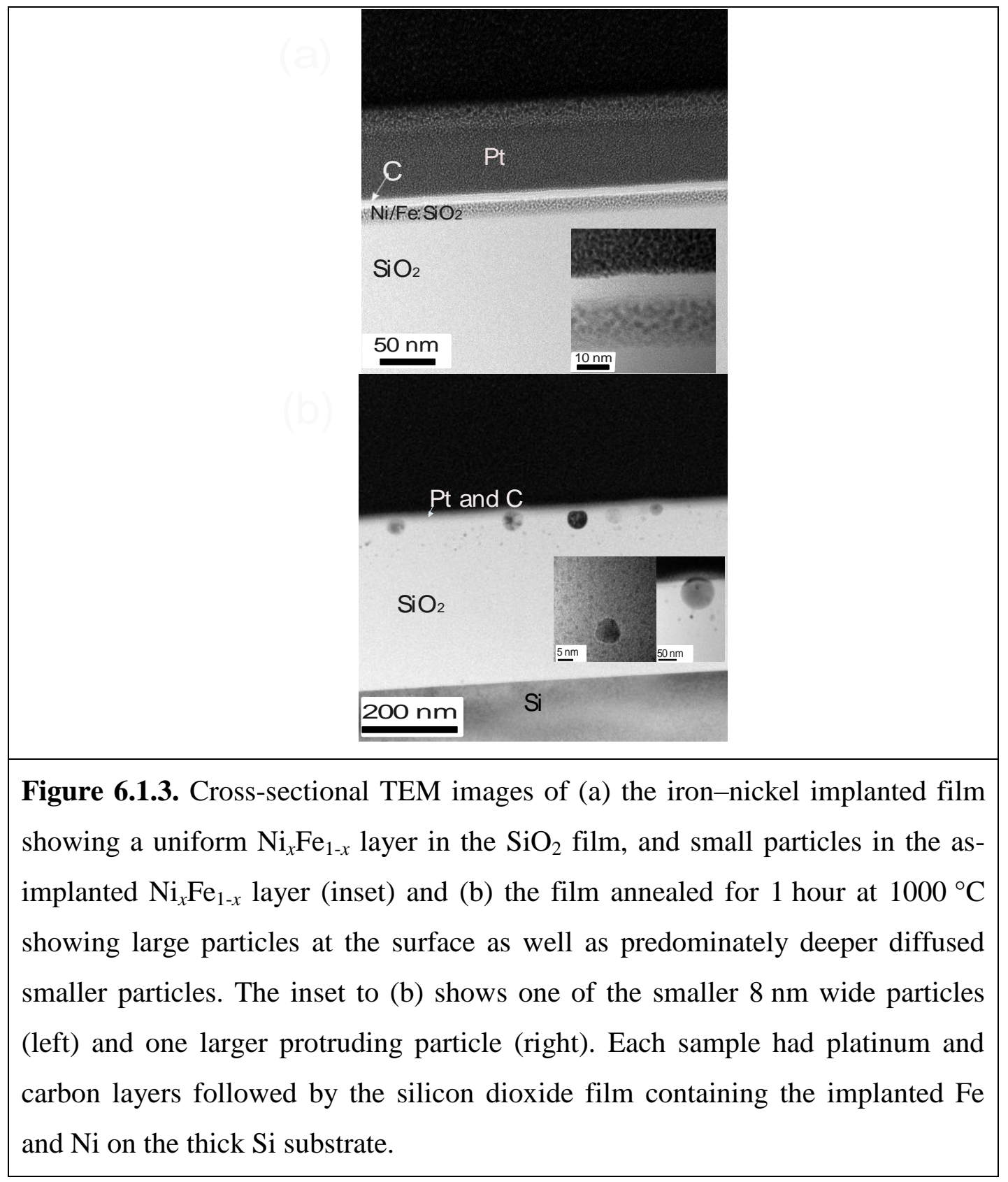

From Figure 6.1.3b and other TEM images (not shown), it was found that the average nanoparticle separation was $\sim 280 \mathrm{~nm}$ and the distance between the nanoparticles ranged from $140 \mathrm{~nm}$ to $393 \mathrm{~nm}$. The smaller nanoparticles have diameters ranging from $2 \mathrm{~nm}$ to $15 \mathrm{~nm}$, with an average diameter of $\sim 6 \mathrm{~nm}$, and a volume fraction of $\sim 12 \%$. A $\sim 8 \mathrm{~nm}$ diameter nanoparticle can be seen in the left inset to Figure 6.1.3b. A small nanoparticle can be seen near the $\mathrm{SiO}_{2} / \mathrm{Si}$ interface. 
There are very few small nanoparticles near the surface and most of the small nanoparticles are predominately in the $60 \mathrm{~nm}$ to $120 \mathrm{~nm}$ depth range with an average depth of $75 \mathrm{~nm}$. Unfortunately, the $\mathrm{Ni}, \mathrm{Fe}$ and $\mathrm{Ni}_{x} \mathrm{Fe}_{1-x}$ lattice parameters are very close so that it is not possible to use electron diffraction to determine the composition of the nanoparticles after 1 hour of EBA.

The bimodal particle sizes and their spatial distribution in the $\mathrm{SiO}_{2}$ films is completely different from previous measurements after Fe implantation at $15 \mathrm{keV}$ and fluencies of $10^{16}$ at. $/ \mathrm{cm}^{2}$ and after 1 hour of EBA where $\sim 10 \mathrm{~nm}$ diameter nanoparticles only formed within the implantation region (J. Leveneur et al., 2011),(Yuan et al., 2009) as well as some at the $\mathrm{Si} / \mathrm{SiO}_{2}$ interface (Kennedy et al., 2012a). In that case, EBA led to sputtering of $\mathrm{Si}$ and oxygen that exposed the $\mathrm{Fe}$ nanoparticles. Some hemispherical core-shell nanoparticles were reported for short EBA times that had $\sim 12 \mathrm{~nm}$ diameter Fe cores and a total diameter of $\sim 30 \mathrm{~nm}$ where the shells were Fe deficient (Yuan et al., 2009). However, the shell disappeared for longer annealing times and only small $\mathrm{Fe}$ and spherical nanoparticles could be seen after 1 hour of EBA (J. Leveneur et al., 2011),(Yuan et al., 2009). Our results are also different from those reported after $60 \mathrm{keV} \mathrm{Ni}$ implantation with a fluence of $5.5 \times 10^{16}$ at. $/ \mathrm{cm}^{2}$ where the magnetic data suggests that the average $\mathrm{Ni}$ nanoparticle diameters did not significantly change from $2.9 \mathrm{~nm}$ after $800{ }^{\circ} \mathrm{C}$ annealing in a vacuum for 1 hour (Amekura et al., 2004b). The nanoparticle sizes are also significantly greater than those reported after $50 \mathrm{keV} \mathrm{Ni}$ implantation into $\mathrm{SiO}_{2}$ with a fluence of $6 \times 10^{16}$ at. $/ \mathrm{cm}^{2}$ followed by 1 hour annealing in a nitrogen atmosphere at $900{ }^{\circ} \mathrm{C}$ (Malik et al., 2012). In that case a bimodal $\mathrm{Ni}$ nanoparticle size distribution was found but the average diameters were $3 \mathrm{~nm}$ and $8 \mathrm{~nm}$.

It is not clear why a bimodal particle size distribution occurs after $\mathrm{Ni}$ and $\mathrm{Fe}$ implantation and EBA that has not been reported for $\mathrm{Ni}$ or $\mathrm{Fe}$ implantation into $\mathrm{SiO}_{2}$. At this stage, a possible growth mechanism can only be speculated upon. The presence of largely isolated particles is possibly an indication of nucleation 
centres that are formed during ion implantation. For example, the implanted film contains $\mathrm{Ni}_{x} \mathrm{Fe}_{1-x}$ regions that could act as nucleation sites for large nanoparticle formation during EBA because $\mathrm{Ni}_{x} \mathrm{Fe}_{1-x}$ has a negative Gibbs free energy of formation that depends on $x$ (Zhang et al., 2013), (Kubaschewski and von Goldbeck, 1949) (Swartzendruber et al., 1991). These nucleation sites will be distributed in the implantation region and the nanoparticles could grow around them via Ostwald ripening and result in large nanoparticles near the surface. The nucleation sites at or close to the surface of the film possibly resulted in the aggregation and growth of large particles growing into spheres with a flattened surface that are seen in the TEM images. Fe or Ni near the tail end of the initial implantation distribution will be at a concentration that is below the solubility limit and hence they could diffuse further into the film and result in the smaller nanoparticles that are observed at greater depths. Any model to describe the bimodal particle size and the different spatial distributions would need to take into account the $\mathrm{Ni}$ and $\mathrm{Fe}$ diffusivities that are completely different; the Ni diffusivity into $\mathrm{SiO}_{2}$ at $1000{ }^{\circ} \mathrm{C} \quad\left(2.5 \times 10^{-16} \mathrm{~cm}^{2} / \mathrm{s}\right.$ (Ramappa and Henley, 1999) (Ghoshtagore, 1969)) is $\sim 2$ orders of magnitude less than that of the respective $\mathrm{Fe}$ diffusivity $\left(3.8 \times 10^{-14} \mathrm{~cm}^{2} / \mathrm{s}\right.$ (Topkaya et al., 2012)). However, it is possible that the diffusivities will be modified in the implantation regions that have high $\mathrm{Ni}$ and $\mathrm{Fe}$ concentrations. A detailed study of the growth mechanism will require a comprehensive study with different fluences and annealing temperatures and times that is beyond the scope of the present study.

\subsubsection{Magnetization measurements}

Magnetization measurements were made on the as-implanted sample and the resultant moment per implanted ion, $m_{\text {ion }}$, is plotted in Figure 6.1.4 against the applied magnetic field, B. The data in Figure 6.1.4 were obtained by first subtracting the silicon substrate contribution from the measured moment, $m$. The resultant moment $m_{\text {ion }}$ was obtained from 


$$
m_{\text {ion }}=\frac{\left(m-\frac{\chi_{\mathrm{Si}} V}{\mu_{0}}\right)}{(n \times A)}
$$

where $\chi_{S i}$ is the measured Si substrate susceptibility $\left(-3.26 \times 10^{-6}\right), V$ is the sample volume, $\mu_{0}$ is the permittivity of free space, $n$ is the fluence in ions per unit area, and $A$ is the sample area.

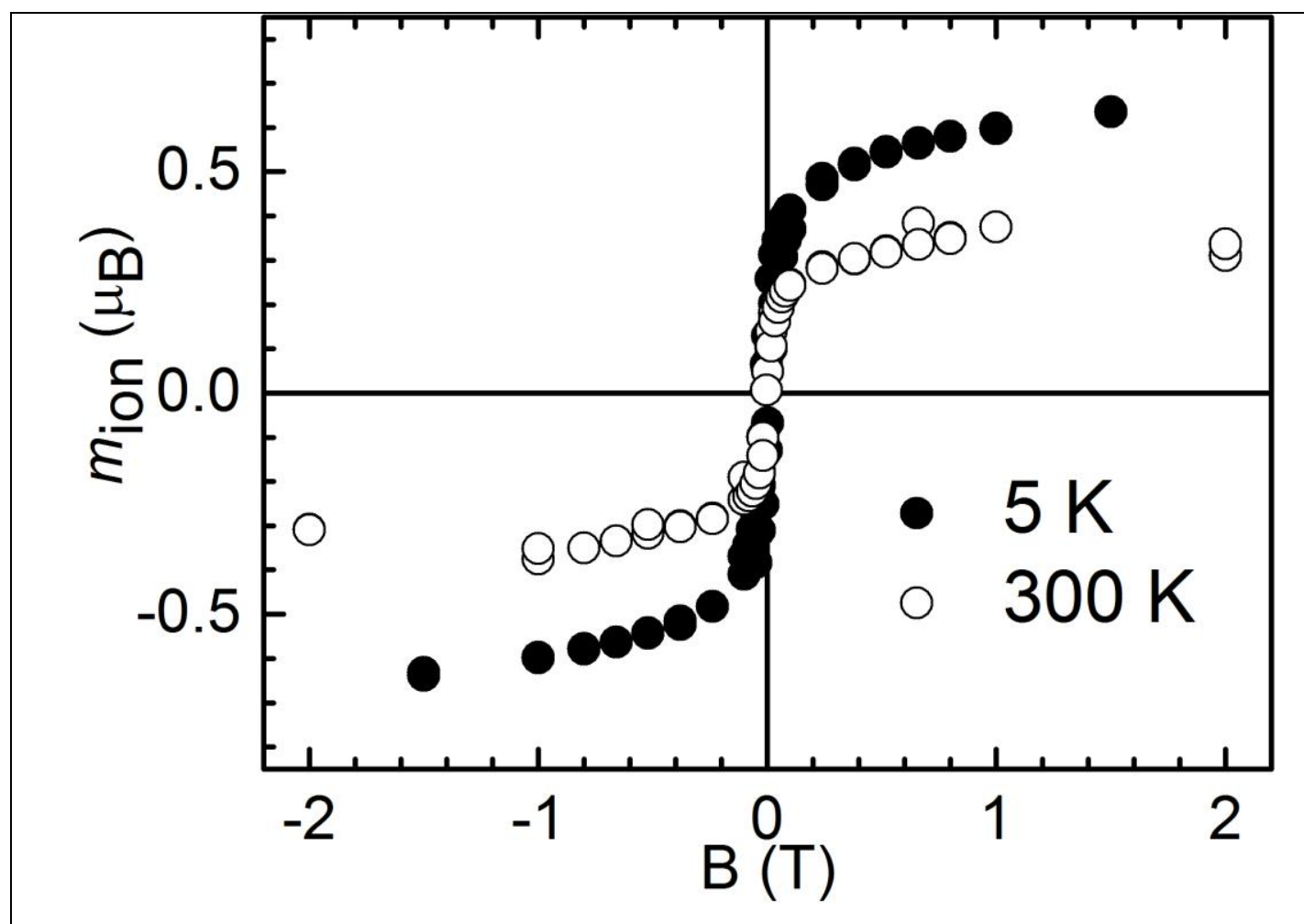

Figure 6.1.4. The moment per implanted ion against the applied magnetic field for the as-implanted film at $5 \mathrm{~K}$ (filled circles) and $300 \mathrm{~K}$ (open circles).

The data are shown below $2 \mathrm{~T}$ because $m_{\text {ion }}$ saturates at the much lower field and there are signal-to-noise issues near $2.4 \mathrm{~T}$ where the measured moment is close to zero owing to the Si substrate contribution.

It is apparent in Figure 6.1.4a that the as-implanted film is ferromagnetic with a Curie temperature above $300 \mathrm{~K} . m_{\text {ion }}$ at $5 \mathrm{~K}$ initially increases to $0.48 \mu_{\mathrm{B}}$ and then slowly increases, while at $300 \mathrm{~K}$ it reaches a much lower value of $0.28 \mu_{\mathrm{B}}$ and then slowly increases with increasing B. This is significantly less than the 
expected low temperature saturation $m_{\text {ion }}$ that is $1.06 \mu_{\mathrm{B}}$ for bulk $\mathrm{Ni}_{x} \mathrm{Fe}_{1-x}$ with $x=0.2$ and it is even less that the expected saturation $m_{\text {ion }}$ of $0.61 \mu_{\mathrm{B}}$ found in bulk ferromagnetic Ni or $2.22 \mu_{\mathrm{B}}$ found in bulk ferromagnetic Fe (Swartzendruber et al., 1991). The gradual increase in $m_{\text {ion }}$ above $\sim 0.2 \mathrm{~T}$ may indicate that there is also a spin-glass (Topkaya et al., 2012) (Ma et al., 2012) that can have a nonsaturating $m_{\text {ion }}$ (Chudnovsky et al., 1986) (Tejada et al., 1991). The significantly lower moment at $5 \mathrm{~K}$ when compared with bulk $\mathrm{Ni}_{x} \mathrm{Fe}_{1-x}$ with a similar $x$ may either be due to some of the magnetic ions not magnetically ordering or the appearance of antiferromagnetic phases (e.g. $\mathrm{NiO}, \mathrm{Fe}_{2} \mathrm{O}_{3}$ ). $m_{\text {ion }}$ values lower than those measured in the bulk have been reported from other studies on small $\mathrm{Ni}_{x} \mathrm{Fe}_{1 \text { - }}$ ${ }_{x}$ nanoparticles (Cushing et al., 2004) (Zhang et al., 1998).

$m_{\text {ion }}$ for the as-implanted sample is plotted in Figure 6.1.5a against temperature at $1 \mathrm{~T}$, where it is apparent that it decreases significantly with increasing temperature. Bulk $\mathrm{Ni}_{x} \mathrm{Fe}_{1-x}$ follows the Bloch temperature dependence that arises from a quadratic magnon dispersion and thermal excitation of magnons that results in a thermal average moment that decreases with increasing temperature. The Bloch function can be written as (Bloch, 1930), as per equation 2.11, $m_{\text {ions }}(\mathrm{T})=m_{\text {ion, }}(0)\left[1-B \times T^{\beta}\right]$, where $m_{\text {ion,s }}$ is the saturation moment per implanted ion, $m_{\text {ion,s }}(0)$ is the saturation moment per implanted ion at $0 \mathrm{~K}$. It is clear in Figure 6.1.5a that $m_{\text {ion }}$ at $1 \mathrm{~T}$ does not follow the temperature dependence expected from Equation 2.11 with $\beta=3 / 2$. This can be seen by a fit to Equation 2.11 that $\beta$ is far lower and it is $\beta=0.38$ where $B=0.053 \mathrm{~K}^{-0.38}$. 


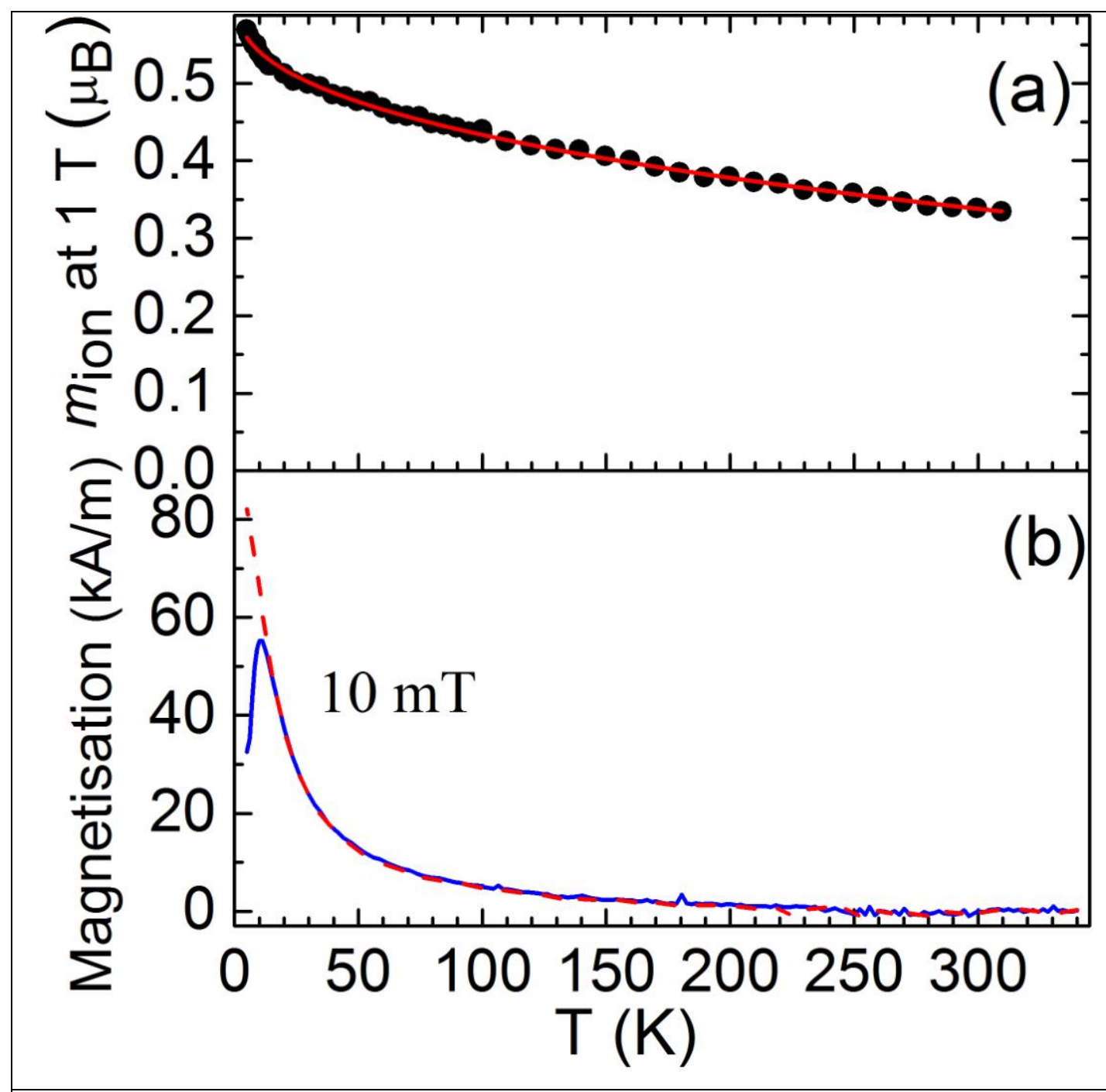

Figure 6.1.5. (a) The moment per implanted ion, $m_{\text {ion }}$, at $1 \mathrm{~T}$ against temperature from the as-implanted film at $1 \mathrm{~T}$ (filled circles). Also shown is a fit to the data using Equation 2.11 (solid curve). (b) The zero-field-cooled (solid curve) and field-cooled (dashed curve) curves at $10 \mathrm{mT}$ for the as-implanted film.

It is known that $\beta$ different from $3 / 2$ can occur in small nanoparticles (Hendriksen et al., 1993)(Aquino et al., 2005)(Maaz et al., 2010),(Zhang et al., 2013). This can arise from the finite number of magnon modes as well as the long wavelength cut off where wavelengths of the allowed magnon modes are limited by the finite nanoparticle size (Hendriksen et al., 1993). This can lead to a 
magnon energy gap that increases $B$ and results in $\beta=2$ (Hendriksen et al., 1993) that has been reported for ferrite nanoparticles with diameters up to $24 \mathrm{~nm}$. However, it has been reported from measurements on Fe nanoparticles capped with $\mathrm{MgF}$ or $\mathrm{MgF}_{2}$ that $\beta \sim 1.5$ for core diameters greater than $\sim 6 \mathrm{~nm}$. $\beta$ was observed to decrease with decreasing core diameter and is low at 0.37 for $\mathrm{MgF}_{2}$ capped nanoparticles with core diameters of $3 \mathrm{~nm}$. At the same time $B$ increases from the bulk value of $3 \times 10^{-6} \mathrm{~K}^{-1.5}$ to as high as $0.6 \mathrm{~K}^{-0.37}$. Thus, the observation of a low $\beta$ and a high $B$ is consistent with the available literature on $\mathrm{Fe}$ nanoparticles. It has been suggested that the lower $\beta$ for small Fe nanoparticles is due to the effect of uncompensated surface spins (Zhang et al., 1998) that can result in temperature dependent spin-glass behaviour (Aquino et al., 2005). The situation in our case is more complicated because the nanoparticles are not free standing. Instead they are concentrated in a narrow layer and in an inhomogeneous $\mathrm{Ni}_{y} \mathrm{Fe}_{1-y} \mathrm{Si}_{z} \mathrm{O}_{n}$ matrix where magnons can propagate between nanoparticles. In one study it was shown that the temperature dependence of $m_{\text {ion }}$ will depend on the boundary conditions as well as the shape and size of the nanoparticles for nanoparticles that are embedded in a matrix when $\beta<1.5$ can occur when the nanoparticle surfaces are not free and magnons propagating throughout the matrix need to be considered (Cojocaru et al., 2014).

In Figure 6.1.5b, the zero-field-cooled and field-cooled magnetizations are plotted against an applied magnetic field of $10 \mathrm{mT}$. The ZFC magnetization has a peak around $13 \mathrm{~K}$ and at higher temperatures the magnetization has a Curie-Weiss temperature dependence. This behaviour is consistent with small superparamagnetic nanoparticles with a blocking temperature of $T_{\mathrm{B}}=13 \mathrm{~K}$. The blocking temperature is defined as (Cullity and Graham, 2011), as per equation 2.12, $T_{\mathrm{B}}=\frac{|K| V}{25 \mathrm{k}_{\mathrm{B}}}$. Using the average radius of $2 \mathrm{~nm}$ found from the TEM analysis and $T_{\mathrm{B}}=13 \mathrm{~K}$, Equation 6.1 .2 is used to show that $|K| \sim 13 \times 10^{4} \mathrm{~J} \mathrm{~m}^{-3}$. This value is comparable to that found for $5 \mathrm{~nm}$ diameter $\mathrm{Ni}_{0.8} \mathrm{Fe}_{0.2}$ nanoparticles (Cushing et 
al., 2004) where $|K|=17 \times 10^{4} \mathrm{~J} \mathrm{~m}^{-3}$. This is greater than that found for $\sim 4 \mathrm{~nm}$ diameter $\mathrm{Ni}_{0.8} \mathrm{Fe}_{0.2}$ nanoparticles that had $T_{\mathrm{B}}=7 \mathrm{~K}$ (Ban et al., 2006) and hence $|K|=7.2 \times 10^{4} \mathrm{~J} \mathrm{~m}^{-3}$ from Equation 6.1.2. The value of $K$ for bulk $\mathrm{Ni}_{0.8} \mathrm{Fe}_{0.2}$ at low temperatures is not known. Based on measurements at room temperature (Bozorth and Walker, 1953), the bulk $K$ might be expected at low temperatures to be in between that of $\mathrm{Fe}\left(K=5.3 \times 10^{4} \mathrm{~J} \mathrm{~m}^{-3}\right.$ (Westerstrand et al., 1975)) and $\mathrm{Ni}$ $\left(K=-12 \times 10^{4} \mathrm{~J} \mathrm{~m}^{-3}\right.$ (Ban et al., 2006),(Westerstrand et al., 1975). Our estimated $|K|$ is slightly outside this range that suggests that there is an enhancement of $|K|$ that has also been reported for other studies of small magnetic nanoparticles (Cushing et al., 2004; Demortière et al., 2011), (Gangopadhyay et al., 1992).

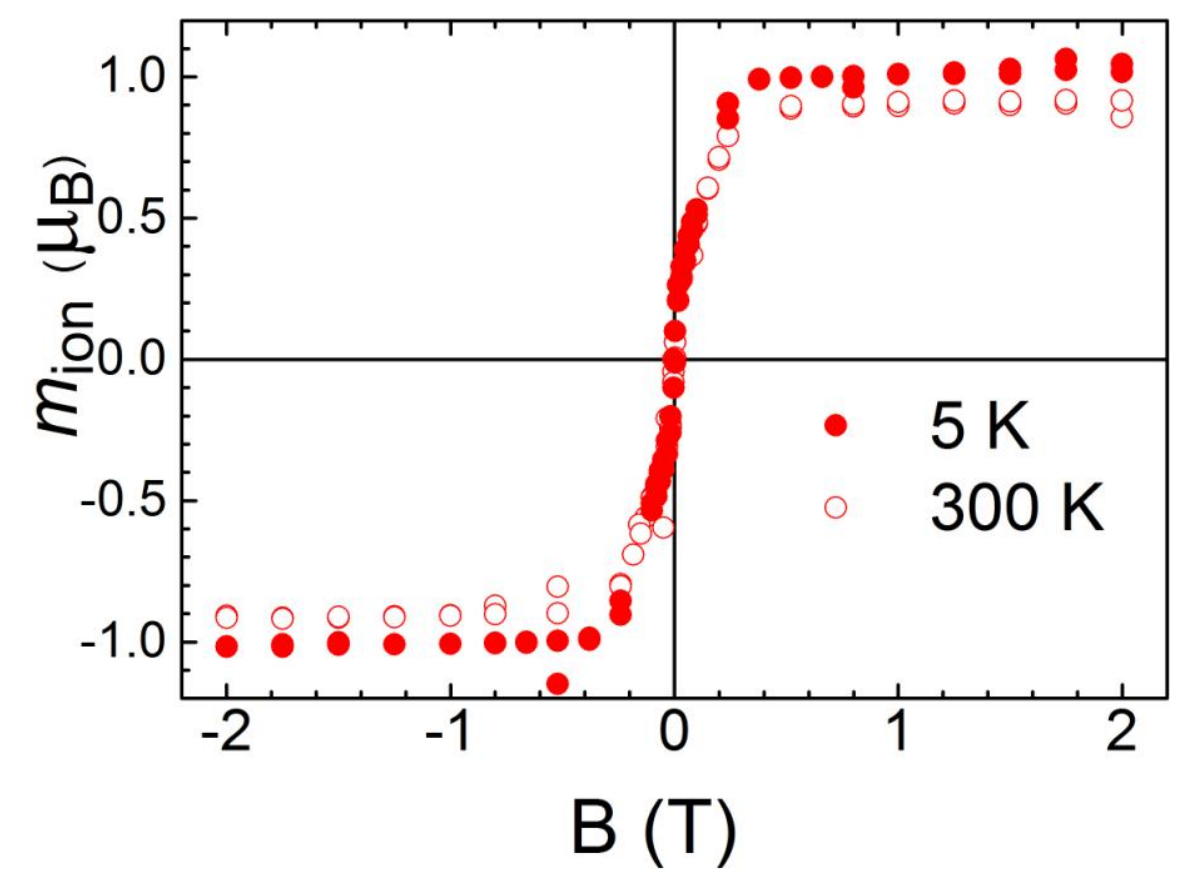

Figure 6.1.6. The moment per implanted ion against the applied magnetic field for the film annealed for 1 hour at $1000{ }^{\circ} \mathrm{C}$ at $5 \mathrm{~K}$ (filled circles) and $300 \mathrm{~K}$ (open circles).

It can be seen in Figure 6.1.6 that EBA at $1000{ }^{\circ} \mathrm{C}$ for 1 hour significantly changes $m_{\text {ion }} \cdot m_{\text {ion }}$ was obtained from the measured moment using equation 6.1.1 
to subtract the silicon substrate contribution. Ferromagnetic order is observed and $m_{\text {ion }}$ still saturates at $\sim 0.2 \mathrm{~T}$, however, $m_{\text {ion }}$ does not change above $\sim 0.2 \mathrm{~T}$ and it is also higher than that found in the as-implanted film. The saturation $m_{\text {ion }}$ is $1.00 \pm 0.09 \mu_{\mathrm{B}}$ at $5 \mathrm{~K}$, which is close to the bulk value of $1.06 \mu_{\mathrm{B}}$ reported for $\mathrm{Ni}_{x} \mathrm{Fe}_{1-x}$ with a similar composition of $x=0.2$ at low temperatures (Swartzendruber et al., 1991). This indicates that all of the implanted $\mathrm{Ni}$ and Fe have magnetically ordered. The saturation moment is considerably larger than that reported in previous studies where other methods have been used to make $\mathrm{Ni}_{x} \mathrm{Fe}_{1-x}$ nanoparticles(Cushing et al., 2004) (XG Li et al., 1997) (Ban et al., 2006). $m_{\text {ion }}$ is slightly lower at $300 \mathrm{~K}$, which is expected due to spin waves that reduce the thermal average moment as the temperature is increased.

In Figure 6.1.7a, the moment per implanted ion $m_{\text {ion }}$ at $1 \mathrm{~T}$ is shown plotted against temperature for the sample annealed for 1 hour. $m_{\text {ion }}$ decreases with temperature but the change from the lowest to the highest temperature is much smaller than that seen for the as-implanted sample (Figure 6.1.7a). It can be seen that $m_{\text {ion }}$ can be fitted to Equation 6.1 .1 with $\beta=0.83$ and $B=4.3 \times 10^{-4} \mathrm{~K}^{-0.83}$. The low value of $\beta$ is surprising since $~ 88 \%$ of the $\mathrm{Ni}$ and $\mathrm{Fe}$ are in isolated nanoparticles with diameters greater than $40 \mathrm{~nm}$ and hence $\beta=1.5$ is expected (Zhang et al., 1998) (Vitta et al., 2008). The departure from $\beta=3 / 2$ may be due to a spin-glass component from the magnetic ions at the surface. This was assumed for ferrite nanopowders where the surface contribution to the magnetization was modelled with a phenomenological term, $\exp \left(-T / T_{\mathrm{f}}\right)$, where $T_{\mathrm{f}}$ is the surface spin freezing temperature and the core was modelled using equation 2.11 (Aquino et al., 2005). Thus, $m_{\text {ion }}$ was fitted to,

$$
m_{\text {ion }}(T)=m_{\text {ion_bulk }}(0)\left[1-B \times T^{\beta}\right]+m_{\text {ion_surface }}(0) \times \exp \left(\frac{-T}{T_{\mathrm{f}}}\right),
$$

It is apparent in Figure 6.1.7a that $m_{\text {ion }}$ from the 1 hour annealed sample can also be fitted to equation 6.1 .3 where $\beta=1.5$ is used that was reported for capped Fe nanoparticles with diameters greater than $6 \mathrm{~nm}$ (Zhang et al., 1998). 
The fitted parameters were $m_{\text {ion,bulk }}(0)=0.992 \mu_{\mathrm{B}}, \quad B=(6.3 \pm 0.4) \times 10^{-6} \mathrm{~K}^{-1.5}$, $m_{\text {ion,surface }}(0)=0.017 \mu_{\mathrm{B}}$, and $T_{\mathrm{f}}=41 \pm 13 \mathrm{~K}$. It is possible to estimate the thickness of the spin disorder shell, $t_{\text {shell, }}$ from $\gamma=m_{\text {ion,bulk }}(0) /\left[m_{\text {ion,bulk }}(0)+m_{\text {ion,surface }}(0)\right]$ where it can be shown that

$$
t_{\text {shell }} \approx\left(1-\gamma^{1 / 3}\right) r
$$

where $r$ is the nanoparticle radius. From the present calculations, $\gamma=0.983$ and hence $t_{\text {shell }}=0.0057 r$. Since the TEM data show that $2 r$ is on average $\sim 56 \mathrm{~nm}$, then $t_{\text {shell }}$ is on average $\sim 0.3 \mathrm{~nm}$. $B$ is slightly greater than that found for bulk $\mathrm{Fe}$ $\left(B=3 \times 10^{-6} \mathrm{~K}^{-3 / 2}\right.$ (Zhang et al., 1998)). However, it is less than that reported for $\mathrm{Ni}_{0.81} \mathrm{Fe}_{0.2}$ nanoparticles with diameter of $20 \mathrm{~nm}$ where $B=13 \times 10^{-6} \mathrm{~K}^{-3 / 2}$ (Vitta et al., 2008)).

It is apparent in Figure 6.1.7b that there are particles with sizes much larger than those that can lead to superparamagnetism with blocking temperatures at or below $340 \mathrm{~K}$. Here the ZFC and FC curves are plotted with an applied magnetic field of $10 \mathrm{mT}$. Hysteresis can be seen up to $340 \mathrm{~K}$ and indicates that any superparamagnetic behaviour would have to occur above $340 \mathrm{~K}$. While there are some small $\sim 10 \mathrm{~nm}$ diameter nanoparticles that may display superparamagnetism, their volume fraction is low and hence the ZFC and FC magnetic data are dominated by the larger nanoparticles with diameters from $44 \mathrm{~nm}$ to $75 \mathrm{~nm}$ that are not superparamagnetic below $340 \mathrm{~K}$. 


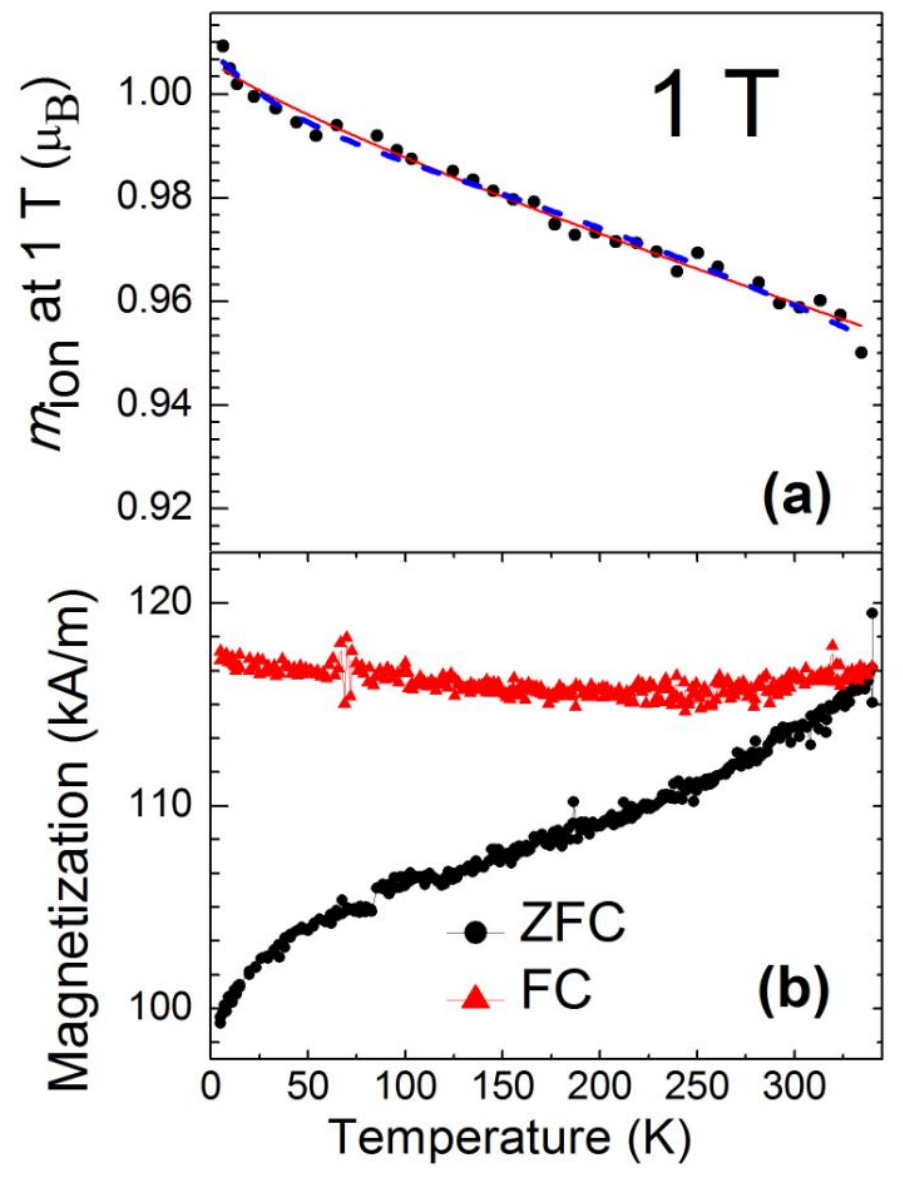

Figure 6.1.7. (a) The moment per implanted ion, $m_{\text {ion }}$, at $1 \mathrm{~T}$ against temperature for the film annealed for 1 hour at $1000{ }^{\circ} \mathrm{C}$ (filled circles). Also shown are fits to Equation 2.11 (solid curve) and Equation 6.1.3 (dashed curve). (b) Zero-fieldcooled (filled circles) and field-cooled (filled up triangles) curves at $10 \mathrm{mT}$ for the annealed film.

Figure 6.1.8 shows a plot of the derivative of the magnetization, $\mathrm{dM} / \mathrm{dH}$, at $5 \mathrm{~K}$ from $-0.8 \mathrm{~T}$ to $0.8 \mathrm{~T}$ for the as-implanted sample and the sample annealed for 1 hour. It was obtained from the measured moment using Equation 6.1.1 and it can be written as,

$$
M / H=\left[\mu_{0} \times m /\left[F \times A \times V_{\text {f.u. }} \times B\right]\right]-\chi_{\mathrm{Si}} \times V / V_{\text {f.u. }}
$$


$\mu_{0} \mathrm{dM} / \mathrm{dH}$ reaches 16 for the 1 hour EBA sample and it a slightly lower value of 12 is found for the as-made sample. This values are significantly less than the expected susceptibility of bulk $\mathrm{Ni}_{x} \mathrm{Fe}_{1-x}$ with a similar $x$ where the susceptibility in greater than 10000. This is likely to be due to the effect of demagnetization.

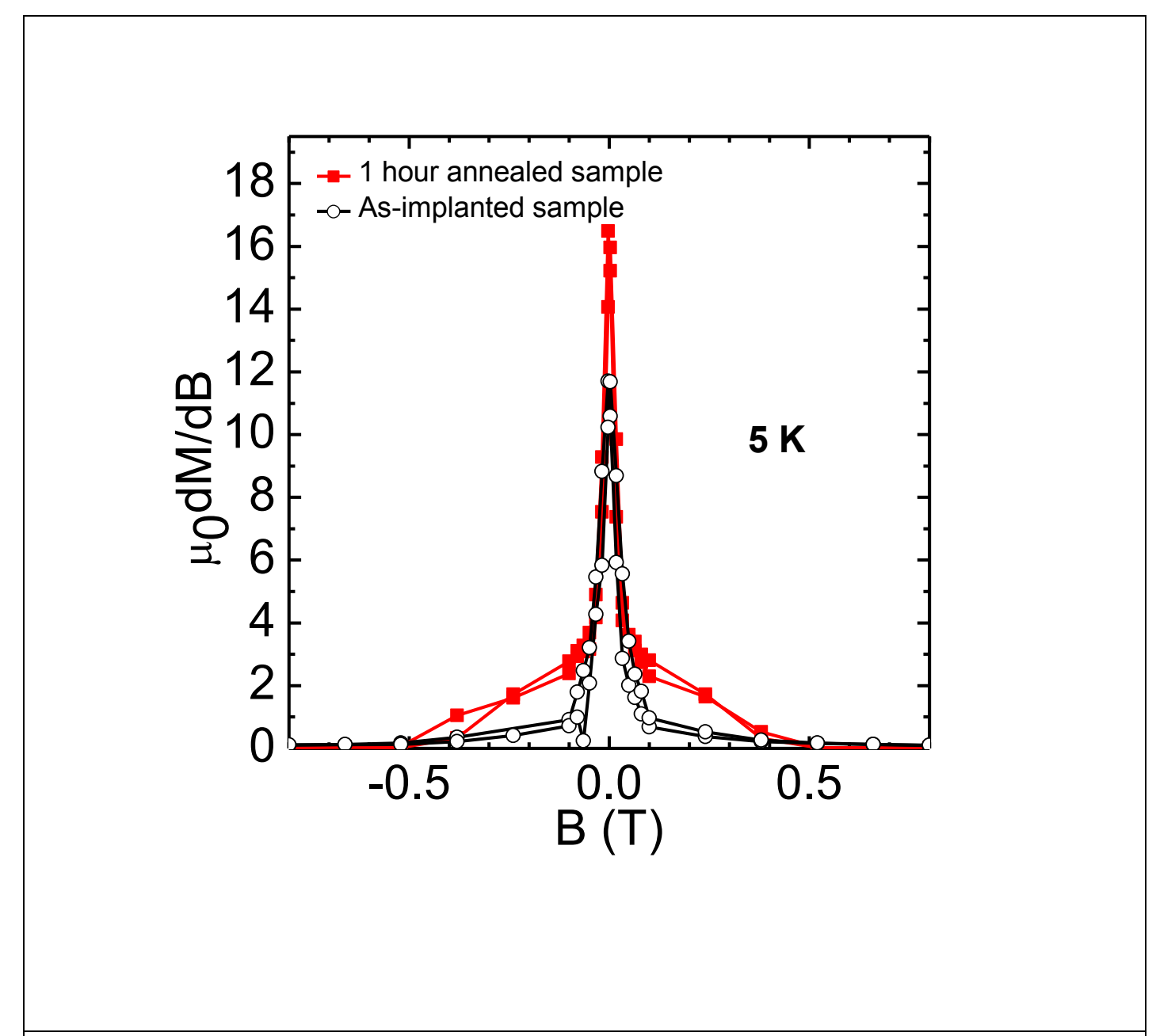

Figure 6.1.8. Plot of $\mu_{0} \mathrm{dM} / \mathrm{dB}$ at $5 \mathrm{~K}$ from $-0.8 \mathrm{~T}$ to $0.8 \mathrm{~T}$ for the as-implanted sample (open circles) and the sample annealed for 1 hour (filled squares).

The as-implanted film shows a gradual slope of the magnetization with decreasing applied field. However, for the annealed film, there appears a change in the gradient of the magnetisation at low fields $(0.08-0.125 \mathrm{~T})$ compared to the behavior at slightly higher fields $(0.25-0.5 \mathrm{~T})$, which suggests that there are two magnetic phases. This could be due to different magnetocrystalline anisotropies 
because the magnetocrystalline anisotropy of permalloy is known to decrease as the concentration of nickel is increased, with a minimum at $75 \% \mathrm{Ni}$ in permalloy that has been slowly cooled (Bozorth, 1951; Bozorth and Walker, 1953) where fast cooling has a higher magnetocrystalline anisotropy. It is also possible that there are some nanoparticles with higher domain wall pinning energies that results in higher saturation fields.

\subsubsection{Conclusions}

In conclusion, magnetic nanoparticles have been made by low energy $\mathrm{Ni}$ and Fe ion implantation into silicon dioxide films with an average Fe fraction of 0.82 . The as-implanted film had superparamagnetic nanoparticles with diameters $\sim 4 \mathrm{~nm}$, as estimated from the TEM data, in a $\sim 20 \mathrm{~nm}$ wide layer near the surface. The blocking temperature was $13 \mathrm{~K}$ and the resultant magnetocrystalline anisotropy energy was $\sim 13 \times 10^{4} \mathrm{~J} \mathrm{~m}^{-3}$ and it is in the range expected for $\mathrm{Ni}_{0.82} \mathrm{Fe}_{0.18}$ nanoparticles. The moment per implanted ion at high magnetic fields is significantly less than that expected for bulk $\mathrm{Ni}_{0.82} \mathrm{Fe}_{0.18}$, which implies that there are some antiferromagnetic phases or magnetic ions that do not magnetically order. The temperature dependence of the high field moment has an exponent $(\beta=0.38)$ that is significantly less than that expected for bulk Fe or $\mathrm{Ni}(\beta=3 / 2)$ or small ferromagnetic nanoparticles where quantum confinement and long wavelength cut off effects can lead to an enhanced exponent $(\beta=2)$. This may be due to the effect of disordered spins at the nanoparticle surface as well as magnon propagation through the implanted layer that is an inhomogeneous composite comprising many nanoparticles in a $\mathrm{Ni}_{y} \mathrm{Fe}_{1-y} \mathrm{Si}_{z} \mathrm{O}_{\mathrm{n}}$ matrix where both of these effects are known to reduce the exponent from $3 / 2$.

Electron beam annealing at $1000{ }^{\circ} \mathrm{C}$ for 1 hour results in a bimodal particle size distribution that is not seen after only $\mathrm{Fe}$ or Ni implantation with comparable energies and annealing times where only small nanoparticles are reported. It is found that there are isolated nanoparticles near the surface with diameters ranging from $35 \mathrm{~nm}$ to $110 \mathrm{~nm}$ that are not superparamagnetic. $\sim 12 \%$ of 
the nanoparticles are small and with sizes ranging from $2 \mathrm{~nm}$ to $15 \mathrm{~nm}$. Most of the small nanoparticles are away from the surface and extend 60-120 nm into the $\mathrm{SiO}_{2}$ film, which was not previously observed after only Fe implantation and EBA for the same duration and where most of the nanoparticles were near the surface and the average diameter was $\sim 6 \mathrm{~nm}$. The saturation moment per implanted ion is similar to that expected for bulk $\mathrm{Ni}_{0.82} \mathrm{Fe}_{0.18}$ and hence all of the $\mathrm{Ni}$ and $\mathrm{Fe}$ have magnetically ordered. It was found that the temperature dependence of the high field moment per implanted ion can be modelled using the Bloch equation with $\beta=3 / 2$ and a nanoparticle shell term from disordered moments that lead to a spinglass with a freezing temperature of $41 \mathrm{~K}$. The average disordered moment thickness is estimated to be $\sim 0.3 \mathrm{~nm}$.

Both the as-implanted and annealed films show a susceptibility that is significantly less than that found in the bulk and there was also evidence for two magnetic phase behaviour. 


\subsection{1 $\mathrm{Ni}_{45} \mathrm{Fe}_{55}$ implanted and annealed}

In the previous section, sequential implantation of iron and nickel with a maximum Ni concentration of 0.22 followed by electron beam annealing lead to the formation of a bimodal particle size distribution and a saturation moment comparable to that of an alloy of iron and nickel. The other interesting $\mathrm{Ni}$ concentration is $x=0.45$ which is known as permalloy 45 that has been reported to have a higher permeability in the lower annealed regime. In this section, dual implantation of nickel to iron with a $\mathrm{Ni}$ to $\mathrm{Fe}$ fluence ratio of 45:55 was performed where it is shown below that $x$ is close to 0.45 . The structural properties and the magnetic properties are studied and compared with the results in the previous section.

\subsubsection{Experimental details}

$\mathrm{Ni}_{x} \mathrm{Fe}_{1-x}$ nanoclusters embedded in silicon dioxide were made using the same low-energy implanter mentioned in section 6.1.1 followed by EBA at 1000 ${ }^{\circ} \mathrm{C}$ under high vacuum. The implantation energy was the same as in section 6.1.1 and it was of $10 \mathrm{keV}$. The implanted nickel fluence was $2 \times 10^{16} \mathrm{at} . / \mathrm{cm}^{2}$ and it was the same as that used in the previous section. ${ }^{56} \mathrm{Fe}+$ was implanted onto the nickelimplanted samples with the fluence of $2.5 \times 10^{16}$ at. $/ \mathrm{cm}^{2}$. The iron to nickel fluence ratio is $80 \%$. D-TRIM simulations were used for depth and concentration profiling at the implantation energy. EBA was used to anneal the sample at $1000^{\circ} \mathrm{C}$ for a duration of 1 hour. The heating rate used was $5^{\circ} \mathrm{C} / \mathrm{s}$. After holding at $1000^{\circ} \mathrm{C}$ for $3600 \mathrm{~s}$, the sample was slowly cooled at a rate of $3{ }^{\circ} \mathrm{C} / \mathrm{s}$ up to $300^{\circ} \mathrm{C}$ and furnace-cooled down to room temperature. RBS measurements were performed using a $2 \mathrm{MeV} \mathrm{He}{ }^{+}$beam and current of $\sim 20 \mathrm{nA}$. The fluences were obtained by fitting using RUMP software. Cross-sectional TEM was performed on samples prepared using FIB lift-out technique (Gianuzzi, 1998). For sample preparation, a 300-nm thick Pt protection layer over a $\sim 10 \mathrm{~nm}$ carbon layer was deposited using the electron-beam deposition facility of the FET Nova Nanolab 
dual-beam FIB system. FIB milling at $5 \mathrm{keV} \mathrm{Ga+} \mathrm{ions} \mathrm{was} \mathrm{used} \mathrm{at} \mathrm{the} \mathrm{final} \mathrm{stage.}$ The TEM study was conducted using the FEI Tecnai TF 20 electron microscope operated at $200 \mathrm{kV}$. Magnetic measurements were made using a magnetic measurements property system from Quantum Design as described in section 3.2.6.

\subsubsection{Structural analysis}

\subsection{D-TRIM simulations}

The results from the D-TRIM simulations performed with ${ }^{58} \mathrm{Ni}^{+}$being implanted into silicon dioxide with an energy of $10 \mathrm{keV}$ followed by the implantation of ${ }^{56} \mathrm{Fe}^{+}$with the same energy can be seen in Figure 6.2.1 ${ }^{1}$. The depth-dependent profile of the resultant ratio of the relative concentration of iron to nickel with depth is also seen in this figure. The simulated average estimated depth of nickel in silicon dioxide is $\sim 15 \mathrm{~nm}$ and the maximum depth is $35 \mathrm{~nm}$. The average straggling depth is $\sim 30 \mathrm{~nm}$ and the depth of the iron implanted into this system with nickel implanted into silicon dioxide is similar, which implies that the implanted layer is a mixture of the iron and nickel. The maximum $\mathrm{Fe}$ concentration is close to $23 \%$ at a depth of $15 \mathrm{~nm}$ and the maximum concentration of $\mathrm{Ni}$ is $18 \%$ in the same region. The Fe fraction varies from $\sim 0.58$ at the surface to 0.50 as the depth is increased. The nearly uniform $\mathrm{Ni}_{x} \mathrm{Fe}_{1-x}$ layer is different from that seen in the $x=0.82$ implanted film where the concentration of Fe was negligible at the surface and the $\mathrm{Fe}$ fraction increased with depth until the maximum Ni concentration was achieved after which it did not significantly change with depth.

\footnotetext{
${ }^{1}$ Figure numbers in section 6.2.1 are contracted for brevity (Actual number is 6.2.1.1).
} 


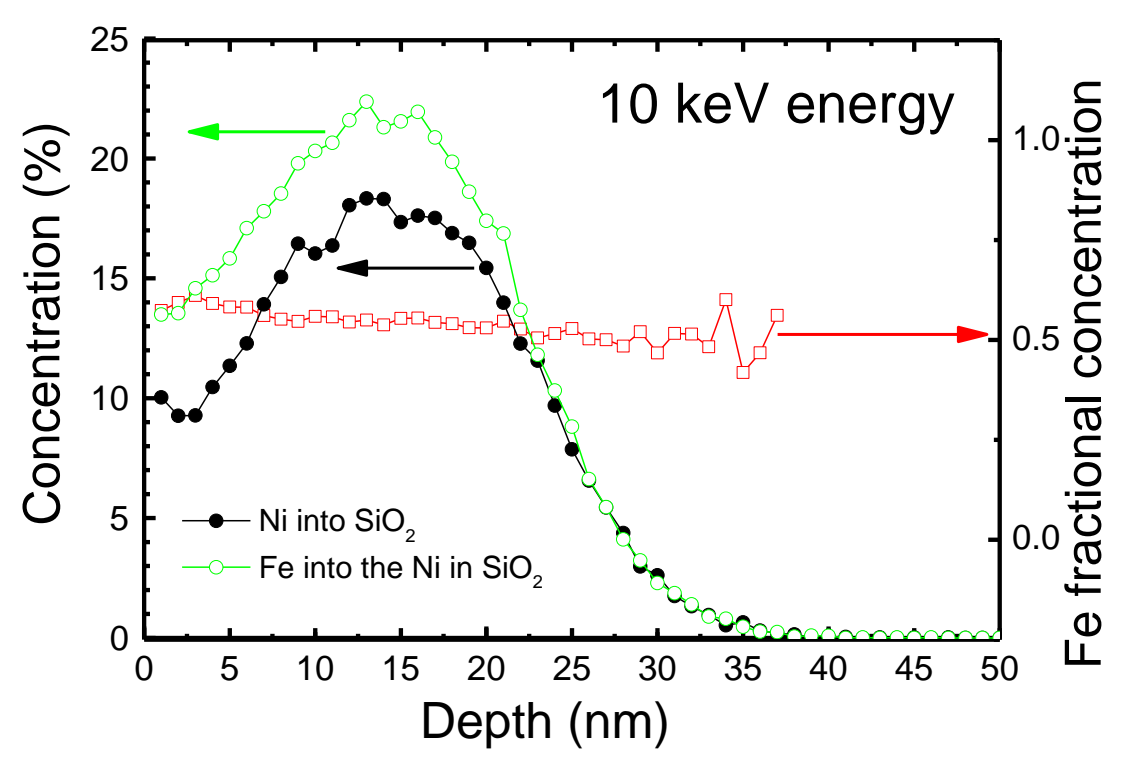

Figure 6.2.1. D-TRIM simulations for the implantation of Ni into silicon dioxide at a fluence of $2 \times 10^{16}$ at. $\mathrm{cm}^{-2}$ (filled circles) and subsequent implantation of $\mathrm{Fe}$ with a fluence of $2.5 \times 10^{16}$ at. $\mathrm{cm}^{-2}$ (open circles) into the Ni-implanted films at $10 \mathrm{keV}$ and the resultant $\mathrm{Fe}$ fraction as a function of depth (open squares). The average implantation depth was $15 \mathrm{~nm}$ and the maximum depth was $35 \mathrm{~nm}$ for the dual implantation.

\subsection{Rutherford backscattering analysis}

Figure 6.2.2 shows the Rutherford backscattering data for the as-implanted film and the film EBA for 1 hour at $1000^{\circ} \mathrm{C}$ and focussing on the combined $\mathrm{Ni}$ and Fe peaks that cannot be resolved. The RBS data at lower energies is similar to that seen for $x=0.82$ and plotted in Figure 6.2.2. The only different was that there is no small peak at $1.17 \mathrm{MeV}$ after EBA, which indicates that there is negligible $\mathrm{Ni}$ or Fe diffusion to the $\mathrm{SiO}_{2} / \mathrm{Si}$ interface. The RBS data were fitted using RUMP software to obtain the total $\mathrm{Ni}$ and Fe fluencies (shown in inset of Figure 6.2.2). They were $4.35 \times 10^{16}$ at. $/ \mathrm{cm}^{2}$ and $4.24 \times 10^{16}$ at. $/ \mathrm{cm}^{2}$ for the as-implanted film and after EBA, respectively. These are the same as the total expected fluence $\left(4.5 \times 10^{16}\right.$ at. $\left./ \mathrm{cm}^{2}\right)$ within the experimental error. Electron beam annealing of $\mathrm{Fe}$ 
implanted films is known to sputter $\mathrm{Si}$ and oxygen and reduce the $\mathrm{SiO}_{2}$ layer thickness by $\sim 11 \mathrm{~nm} /$ hour for iron implantation with a fluence of $1 \times 10^{16} \mathrm{at} . / \mathrm{cm}^{2}$ (Kennedy et al., 2012) (Ramappa and Henley, 1999). This is likely to also occur for the $\mathrm{Ni}_{x} \mathrm{Fe}_{1-x}$ films after EBA but it cannot be resolved in Figure 6.2.2.

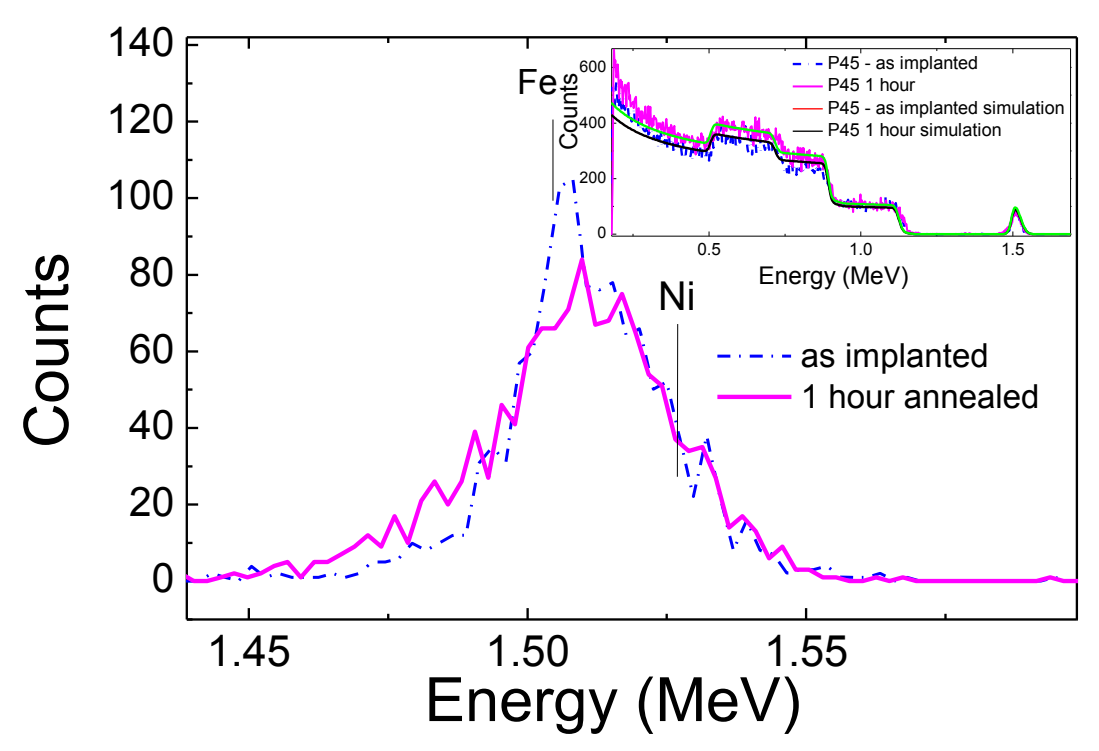

Figure 6.2.2. Rutherford backscattering data focusing on the $\mathrm{Fe}$ and $\mathrm{Ni}$ combined peaks for the as-implanted sample (dashed curve) and the sample that has been electron-beam annealed at $1000^{\circ} \mathrm{C}$ for 1 hour (solid curve). Inset: RBS data over all energies and the simulations.

After annealing the $\mathrm{Ni} / \mathrm{Fe}$ RBS peak has slightly broadened to lower energy indicating that there has been some minor $\mathrm{Ni}$ and $\mathrm{Fe}$ diffusion into the $\mathrm{SiO}_{2}$ film and also possibly some intermetallic diffusion (Anderson et al., 1997). This can be contrasted with the previous study where EBA for the same time and temperature led to a reduction in the $\mathrm{Ni} / \mathrm{Fe}$ peak energy to $1.491 \mathrm{MeV}$ and asymmetry with intensity to energies down to as low as $1.37 \mathrm{MeV}$ and indicating significant diffusion into the $\mathrm{SiO}_{2}$ film. As we show below from the TEM data, this difference may be due to the shape of the nanoparticles that form after EBA 
for $x=0.45$ as well as the absence of the formation of small nanoparticles further into the $\mathrm{SiO}_{2}$ film.

\subsection{Transmission electron microscopy}

The TEM image from the as-implanted sample is shown in Figure 6.2.3a. There are $\mathrm{Pt}$, carbon, implanted $\mathrm{Ni}_{x} \mathrm{Fe}_{1-x}$, and $\mathrm{SiO}_{2}$ layers that can be seen from the top to the bottom of the image. The $\mathrm{Ni}_{x} \mathrm{Fe}_{1-x}$ layer is $24 \mathrm{~nm}$ deep, which is comparable to the straggling depth seen in the D-TRIM simulations. It is apparent that the $\mathrm{Ni}_{x} \mathrm{Fe}_{1-x}$ layer is inhomogeneous and there are nanoparticles with sizes ranging from $2 \mathrm{~nm}$ to $5 \mathrm{~nm}$ and where the average size is $\sim 4 \mathrm{~nm}$. The appearance of small particles with an average particle size of $\sim 4 \mathrm{~nm}$ for $x=0.82$ (see Figure 6.1.3). As mentioned in section 6.1 nanoparticles are expected to form when the Fe and Ni concentration are above the solubility limit.

Figure 6.2.3b shows a TEM image from the film after EBA for 1 hour. Large isolated nanoparticles are evident close to the surface. The large particles are $\sim 91 \mathrm{~nm}$ and $\sim 190 \mathrm{~nm}$ wide and $190 \mathrm{~nm}$ to $117 \mathrm{~nm}$ deep. Both nanoparticles are asymmetrical where one nanoparticle is nearly hemispherical and it contains a shell. More large asymmetric nanoparticles close to the surface can be seen in Figure $6.2 .3 \mathrm{c}$ along with a smaller nanoparticle that has a diameter of $\sim 14 \mathrm{~nm}$ as can be seen in the inset. From these and other TEM images we find that the large asymmetric nanoparticles penetrate from $25 \mathrm{~nm}$ to $125 \mathrm{~nm}$ into the film. Figure 6.2.3d shows an HRTEM image of the large core/shell nanoparticle seen in Figure 6.2.3b and elemental mapping of $\mathrm{Fe}$ and $\mathrm{Ni}$ in this nanoparticle. The elemental mapping of $\mathrm{Fe}$ and $\mathrm{Ni}$ show that the particle consists of the $\mathrm{Ni}_{x} \mathrm{Fe}_{1-x}$ core and a $\mathrm{Fe}_{1-y} \mathrm{Si}_{y} \mathrm{O}_{x}$ shell that does not contain $\mathrm{Ni}$. 


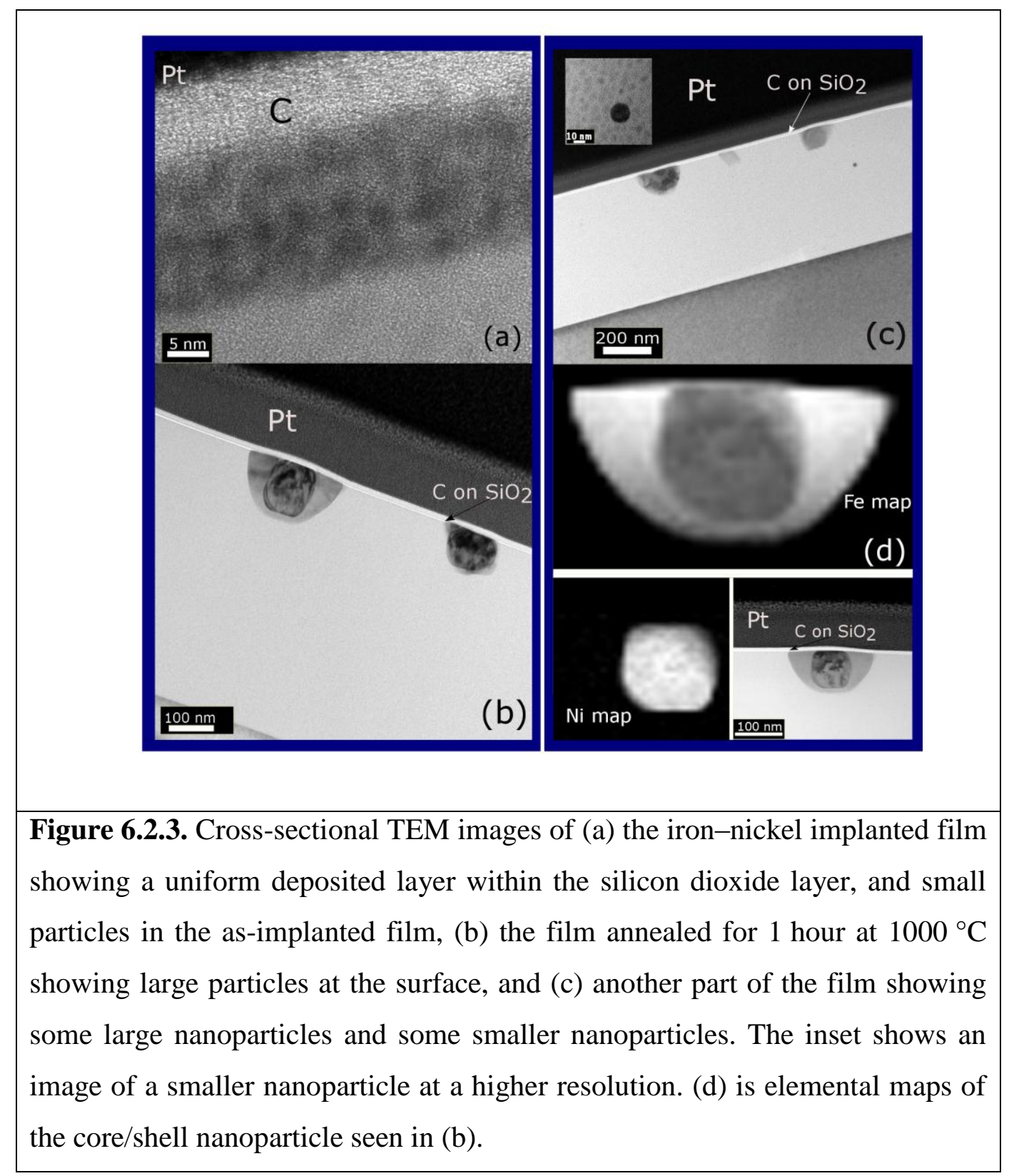

The TEM results after EBA are different from those found for $x=0.82$. While the $x=0.82$ sample also showed large isolated nanoparticles near the surface, they were spherical and only slightly truncated and there were no core/shell nanoparticles. Furthermore, there was a bimodal particle size distribution with smaller $\sim 10 \mathrm{~nm}$ diameter nanoparticles extending $\sim 75 \mathrm{~nm}$ into the film for $x=0.82$ that is not evident for the $x=0.45$ sample. The different 
nanoparticle morphologies may be due to the nanoparticle growth mechanism as well as the different $\mathrm{Ni}$ ratios and as-implanted depth concentration profiles. It was suggested in the previous section that nanoparticle growth occurs around small $\mathrm{Ni}_{x} \mathrm{Fe}_{1-x}$ nanoparticles because $\mathrm{Ni}_{x} \mathrm{Fe}_{1-x}$ has a negative Gibbs free energy of formation (Chase, 1998) that depends on $x$ where it is expected to be a minimum for $x=0.3$. The optimal nucleation sites with $x \sim 0.3$ will be distributed in the $\mathrm{Ni}_{x} \mathrm{Fe}_{1-x}$ layer and the nanoparticles will grow by Oswald ripening. A low nucleation site density can account for the large size and the small number density of larger nanoparticles near the surface where the depth penetration range for $x=0.45$ and $x=0.82$ is similar. This can lead to $\mathrm{Ni}_{x} \mathrm{Fe}_{1-x}$ nanoparticles with $x \sim 0.3$. However, the $x=0.45$ sample has excess $\mathrm{Fe}$ and hence the growth process can occur via the initial formation of $\mathrm{Ni}_{x} \mathrm{Fe}_{1-x}$ followed by the excess Fe forming a $\mathrm{Fe}$ rich shell. The $x=0.45$ as-implanted sample also has a large $\mathrm{Fe}$ and $\mathrm{Ni}$ concentration at the surface (see Figure 6.2.1) when compared with the $x=0.82$ sample where the Fe concentration is close to zero near the surface and this could drive the formation of asymmetric nanoparticles that are truncated at the surface. This could also explain why the RBS data for the $x=0.55$ sample does not show as much diffusion as that seen for $x=0.82$. This is because of hemispherical or surface truncated samples will have a high $\mathrm{Ni}-\mathrm{Fe}$ concentration near the surface and hence the peak $\mathrm{Ni}$ and Fe concentration will be close to the surface. This can be compared with spherical nanoparticles where the peak concentration is further into the $\mathrm{SiO}_{2}$ film and at the nanoparticle radius.

\subsubsection{Magnetization}

Magnetization measurements were done on the as-implanted film and the moment per implanted ion, $m_{\text {ion }}$, is plotted in Figure 6.2.4 against the applied magnetic field at $5 \mathrm{~K}$ and $300 \mathrm{~K} . m_{\text {ion }}$ was obtained from the measured moment using equation 6.1.1. The measured moment is noisy above $\sim 2 \mathrm{~T}$ because it is close to zero due to the contribution from the silicon substrate and hence the data range is limited to $\pm 2 \mathrm{~T}$ in Figure 6.2.4. The magnetic field dependence of $m_{\text {ion }}$ is 
consistent with the appearance of ferromagnetism that clearly persists far above $300 \mathrm{~K}$. This can be attributed to the $\mathrm{Ni}_{x} \mathrm{Fe}_{1-x}$ nanoparticles where the bulk Curie temperature is $869 \mathrm{~K}$. The saturation moment, $m_{\mathrm{ion}, \mathrm{s}}$, is $0.52 \mu_{\mathrm{B}}$, which is significantly less than the bulk value of $1.8 \mu_{\mathrm{B}}$ for $x=0.45$ [Li 1997] or even that for bulk $\mathrm{Ni}\left(0.61 \mu_{\mathrm{B}}\right)$. Reduced $m_{\mathrm{ion}, \mathrm{s}}$ values are observed in other nanoparticle systems due to disordered moments near the nanoparticle surface. It our case it is also likely that there are some unordered magnetic moments as well as antiferromagnetic phases.

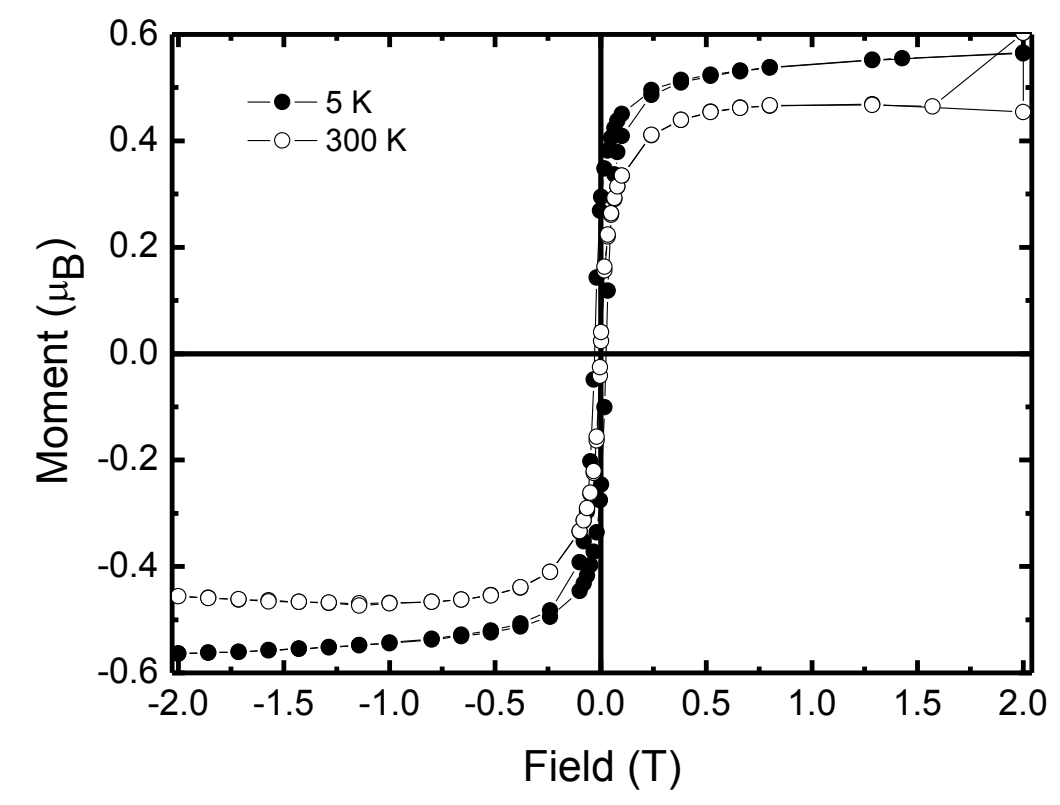

Figure 6.2.4. The moment per implanted ion against the magnetic field for the as-implanted film at $5 \mathrm{~K}$ (filled circles) and $300 \mathrm{~K}$ (open circles).

The saturation moment is plotted against temperature as shown in Figure 6.2.5a for the as-implanted film. The magnetization from bulk $\mathrm{Ni}_{x} \mathrm{Fe}_{1-x}$ reduces as the temperature increases due to spin-waves and for bulk $\mathrm{Ni}_{x} \mathrm{Fe}_{1-x} m_{\mathrm{ion}, \mathrm{s}}$ can be written using equation 2.11, $m_{\mathrm{ion}, \mathrm{s}}(\mathrm{T})=m_{\mathrm{ion}, \mathrm{s}}(0)\left[1-B \times T^{\beta}\right]$. However, it is apparent in Figure 6.2.5a that Equation 2.11 cannot describe the data due to an 
upturn at low temperatures. This was not observed for $x=0.82$ where $m_{\mathrm{ion}, \mathrm{s}}$ could be fitted to Equation 2.10 with $\beta=0.38$, which is far below the $\beta=3 / 2$ found in bulk $\mathrm{Ni}_{x} \mathrm{Fe}_{1-x}$. The appearance of an upturn in $m_{\mathrm{ion}, \mathrm{s}}$ at low temperatures was discussed in section 6.1 in relation to the annealed $x=0.82$ sample. It indicates the presence of a spin disordered shell (Chudnovsky et al., 1986) (Tejada et al., 1991). It can be seen in Figure 6.2.5a that $m_{\mathrm{ion}, \mathrm{s}}$ can be fitted using Equation 6.1.3 where $m_{\text {ion,bulk }}(0)=(0.532 \pm 0.002) \mu_{\mathrm{B}}, \quad B=(2.43 \pm 0.07) \times 10^{-5} \quad \mathrm{~K}^{3 / 2}, \quad \beta=3 / 2$, $m_{\text {ion,surface }}(0)=(0.041 \pm 0.004) \mu_{\mathrm{B}}$, and $T_{\mathrm{f}}=(17 \pm 3) \mathrm{K} . T_{\mathrm{f}}$ is lower than that found for the annealed $x=0.82$ sample. The value of $B$ is larger than that reported in bulk Fe ( $D=3 \times 10^{-6} \mathrm{~K}^{3 / 2}$ [(Yu et al., 2008)]). It is also larger than that found in the previous section for $\mathrm{Ni}_{x} \mathrm{Fe}_{1-x}$ nanoparticles with an average diameter of $56 \mathrm{~nm}$ and where $B=(6.3 \pm 0.4) \times 10^{-6} \mathrm{~K}^{-1.5}$. It is $\sim 1.87$ times higher than that reported for $\mathrm{Ni}_{0.8} \mathrm{Fe}_{0.2} 20$ $\mathrm{nm}$ diameter nanoparticles $\left(D=1.3 \times 10^{-5} \mathrm{~K}^{3 / 2}\right.$ [(Yu et al., 2008)]) and shows that $\mathrm{D}$ increases with decreasing particle size.

It is possible to estimate the thickness of the spin-disordered shell region, $t_{\text {shell }}$, using equation 6.1.4. This leads to $t_{\text {shell }}=0.027 r$. Using the average radius of $\sim 2 \mathrm{~nm}$ found from the TEM analysis it is possible to estimate that $t_{\text {shell }}$ is small and $t_{\text {shell }}=0.05 \mathrm{~nm}$. This is smaller than that seen for the annealed $x=0.82$ sample and indicates disorder of some of the uncompensated spins in the shell. 


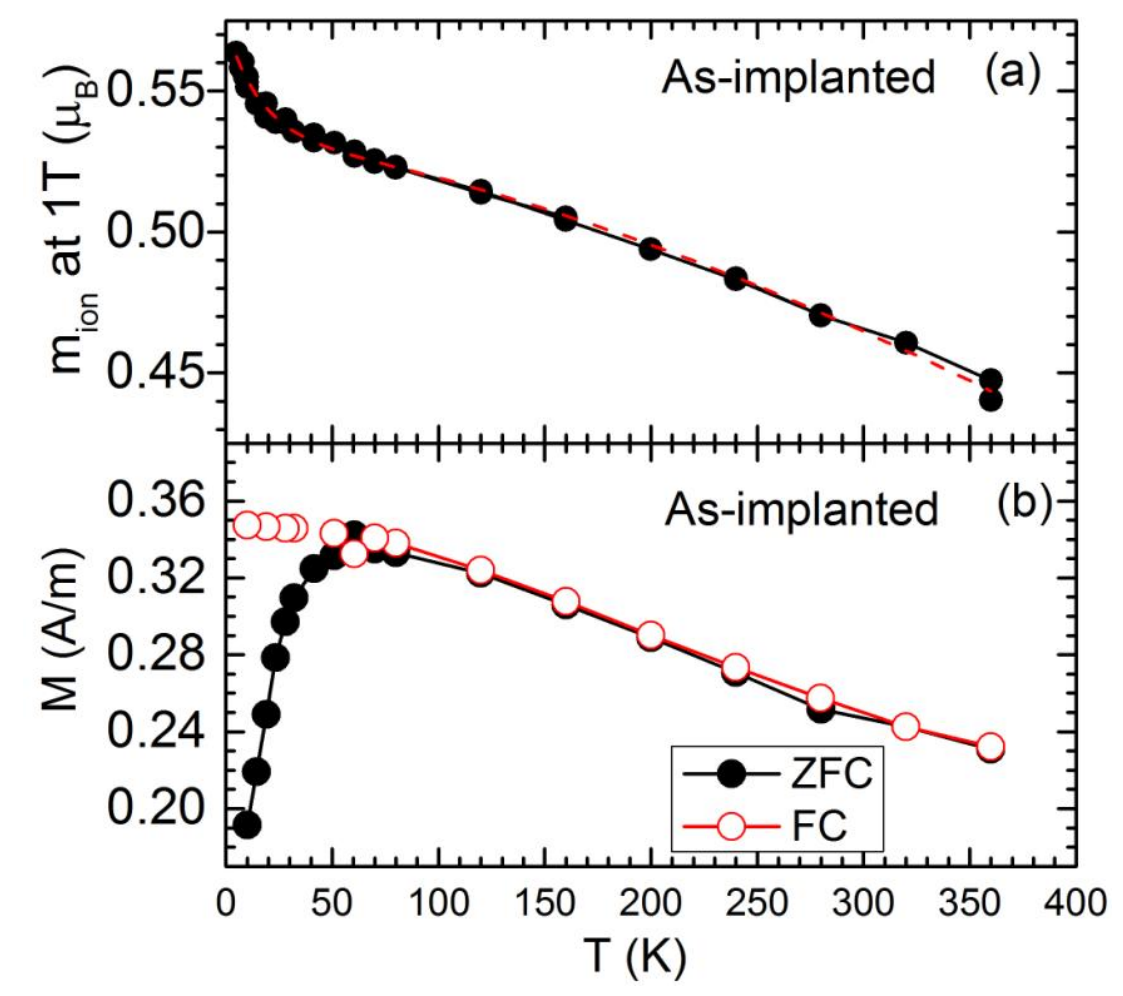

Figure 6.2.5: Plot of the (a) moment per implanted ion at $1 \mathrm{~T}$ against temperature, and (b) the ZFC (open circles) and FC (filled circles) magnetization at $10 \mathrm{mT}$ from the as-implanted sample. Also shown in (a) is a fit to Equation 6.1.3.

The ZFC and FC $m_{\text {ion }}$ for the as-implanted film are plotted for an applied magnetic field of $10 \mathrm{mT}$ in Figure 6.2.5b. The temperature dependence of the ZFC-FC $m_{\text {ion }}$ is consistent with the appearance of superparamagnetism where the blocking temperature, $T_{\mathrm{B}}$, is $60 \mathrm{~K}$. Using the average radius of $\sim 2 \mathrm{~nm}$ found from the TEM analysis and $T_{\mathrm{B}}=60 \mathrm{~K}$, we use Equation 2.12 to show that $|K|=62 \times 10^{4}$ $\mathrm{J} \mathrm{m}^{-3}$. This is significantly larger the $|K|$ range found in bulk $\mathrm{Ni}_{x} \mathrm{Fe}_{1-x}$ where as noted in section $6.1 K$ ranges from that of $\mathrm{Fe}\left(K=5.3 \times 10^{4} \mathrm{~J} \mathrm{~m}^{-3}\right)$ to that of $\mathrm{Ni}$ $\left(K=-12 \times 10^{4} \mathrm{~J} \mathrm{~m}^{-3}\right)$. As mentioned in section 6.1 enhancements of $|K|$ are known to occur in small nanoparticles. $|K|$ is larger than that previously found for $x=0.82$ 
made using the sample dual implantation method where $|K|=13 \times 10^{4} \mathrm{~J} \mathrm{~m}^{-3}$ and hence $|K|$ for similar nanoparticles sizes made using our method is larger for higher $x$.

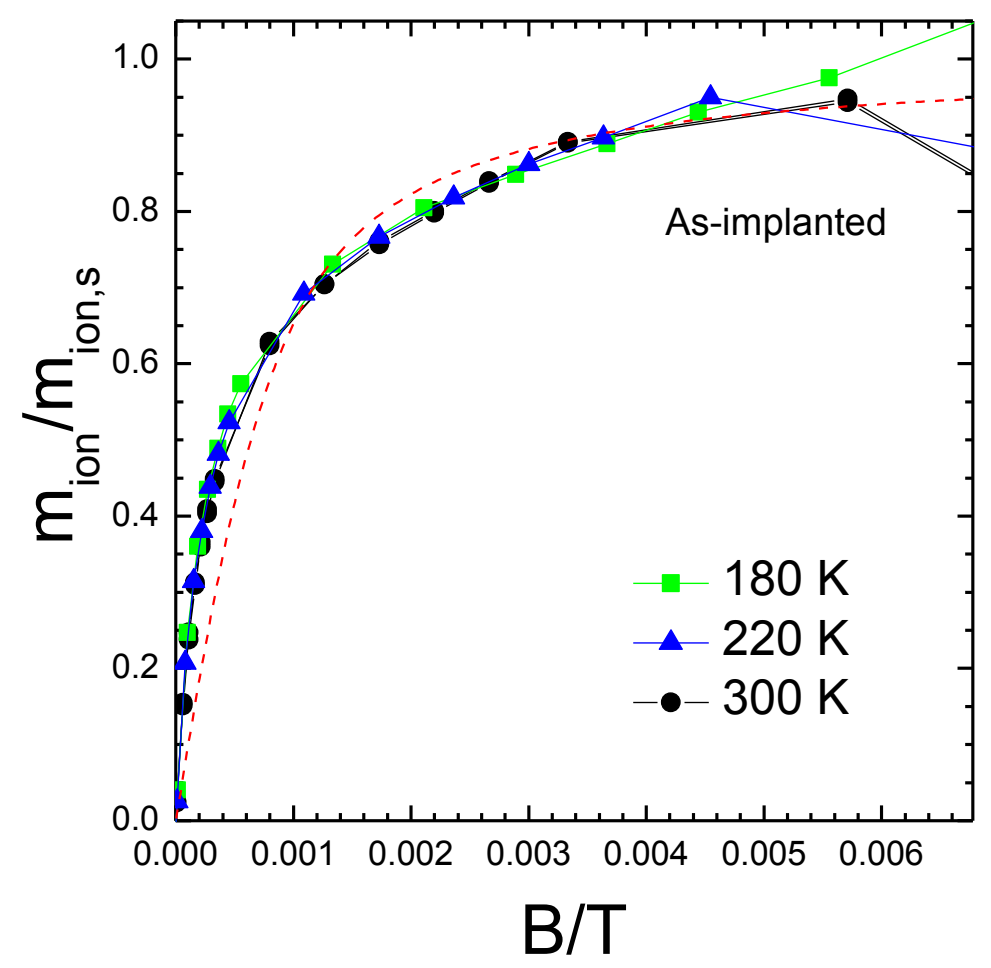

Figure 6.2.6: Plot of the moment per f.u. divided by the saturation moment against the applied magnetic field divided by the temperature for the asimplanted sample at $300 \mathrm{~K}$ (filled circles), $220 \mathrm{~K}$ (filled up triangles) and $180 \mathrm{~K}$ (filled circles). Also shown is a Langevin fit to the data (dashed curve).

We show in Figure 6.2.6 that the reduced moment, $m_{\text {ion }} / m_{\text {ion,s, }}$ at three different temperature above $T_{\mathrm{B}}$ can be plotted on a common curve against $B / T$, where $B$ is the applied magnetic field. This is expected for a system of superparamagnetic particles, where the reduced moment curve is defined by the Langevin function, 


$$
m_{\mathrm{ion}}(B) / m_{\mathrm{ions}}=\operatorname{coth}[\eta]-1 / \eta,
$$

where $\eta=m_{\mathrm{p}} \times V_{\mathrm{p}} \times B / \mathrm{k}_{\mathrm{B}} T$, and $m_{\mathrm{p}}$ is the nanoparticle moment. The data in Figure 6.2.6 were fitted using Equation 6.2.1 where it can be seen that the fit is not perfect, which is likely to be due to a distribution in the nanoparticle sizes. From the fit in Figure 6.2.6 we obtain $m_{\mathrm{p}}=4200 \mu_{\mathrm{B}}$. The nanoparticle radius can be estimated from $r=\left[\left[3 \times m_{\mathrm{p}} \times V_{\text {f.u. }}\right] /\left[4 \times \pi \times m_{\text {ion, s }}\right]\right]^{1 / 3}$, where $V_{\text {f.u. }}$ is the volume per formula unit. This results in $r=2.8 \mathrm{~nm}$, which is comparable to the average $r$ estimated from the TEM data.

The magnetization measurements from the sample that was EBA at $1000^{\circ} \mathrm{C}$ for 1 hour is shown in Figure 6.2.7. The saturation moment per ion is 0.78 $\mu_{\mathrm{B}}$ at $5 \mathrm{~K}$ and it is only slightly lower at $300 \mathrm{~K}$, which indicates that the Curie temperature is significantly above $300 \mathrm{~K}$. However, $m_{\text {ion, s }}$ is significantly lower than that expected for bulk $\mathrm{Ni}_{x} \mathrm{Fe}_{1-x}$ with $x=0.45$ where $m_{\mathrm{ion}, \mathrm{s}}=1.8 \mu_{\mathrm{B}}$ and it is larger than that expected for bulk $\mathrm{Ni}\left(m_{\mathrm{ion}, \mathrm{s}}=0.61 \mu_{\mathrm{B}}\right)$. This can be contrasted with section 6.1 for $x=0.82$ and where $m_{\mathrm{ion}, \mathrm{s}}$ was comparable to that expected for bulk $\mathrm{Ni}_{x} \mathrm{Fe}_{1-x}$ and close to that expected for a mixture of $\mathrm{Fe}$ and $\mathrm{Ni}$ with $18 \% \mathrm{Fe}$. A lower $m_{\mathrm{ion}, \mathrm{s}}$ can occur in small nanoparticles due to disordered moments at and near the nanoparticle surface. However, the size of our nanoparticles is too large for this to occur and as we show below the moment contribution for the shell is small and implies a very small moment disordered region. It is possible that $m_{\mathrm{ion}, \mathrm{s}}$ is lower than expected for $x=0.45$ due to some of the Fe not magnetically ordering or antiferromagnetically ordering. This is consistent with the observation from the TEM data that there is a $\mathrm{Fe}_{1-y} \mathrm{Si}_{y} \mathrm{O}_{x}$ shell and this shell could order antiferromagnetically. One possible scenario is that the nanoparticles have $x$ lower than that expected from the implantation fluence ratios and the remaining $\mathrm{Fe}$ is in $\mathrm{Fe}_{1-y} \mathrm{Si}_{y} \mathrm{O}_{z}$ shells or does not magnetically order. In this case it is possible to estimate the Fe fraction, $x_{1}$, in the $\mathrm{Ni}_{1-x_{1}} \mathrm{Fe}_{x_{1}}$ nanoparticles from, 


$$
x_{1}=\left[m_{\mathrm{ion}, \mathrm{s}}-x_{\mathrm{N} i} \times a\right] /\left[m_{\mathrm{ion}, \mathrm{s}}+x_{\mathrm{Ni}} \times b\right]
$$

where $x_{\mathrm{Ni}}$ is the implanted $\mathrm{Ni}$ fraction $(0.45) . m_{\mathrm{ion}, \mathrm{s}}$ from $x=0$ to 0.55 is approximately linear where $m_{\mathrm{ion}, \mathrm{s}}=a+b \times x$, with $\mathrm{a}=0.61 \mu_{\mathrm{B}}$ and $\mathrm{b}=2.18 \mu_{\mathrm{B}}$. Using the measured $m_{\mathrm{ion}, \mathrm{s}}=0.78 \mu_{\mathrm{B}}$ we find that $x_{1}=0.29$ and hence the expected moment per ion in the $\mathrm{Ni}_{0.71} \mathrm{Fe}_{0.29}$ nanoparticles, $m_{\text {inns }}$ is $1.24 \mu_{\mathrm{B}}$. The resultant $\mathrm{Ni}_{0.71} \mathrm{Fe}_{0.29}$ fraction is obtained from $m_{\mathrm{ions}} / m_{\mathrm{ions} s_{1}}=0.63$. Thus, in this scenario the growth of $\mathrm{Ni}_{1-x_{1}} \mathrm{Fe}_{x_{1}}$ nanoparticles with $x_{1}=0.29$ is thermodynamically favourable and the remaining $\mathrm{Fe}$ is either in the $\mathrm{Fe}_{1-y} \mathrm{Si}_{y} \mathrm{O}_{z}$ shell or not magnetically ordered. The value of $x_{1}=0.29$ is in the region where the Gibbs free energy of formation is a minimum and hence it is consistent with the nanoparticle growth mechanism discussed earlier.

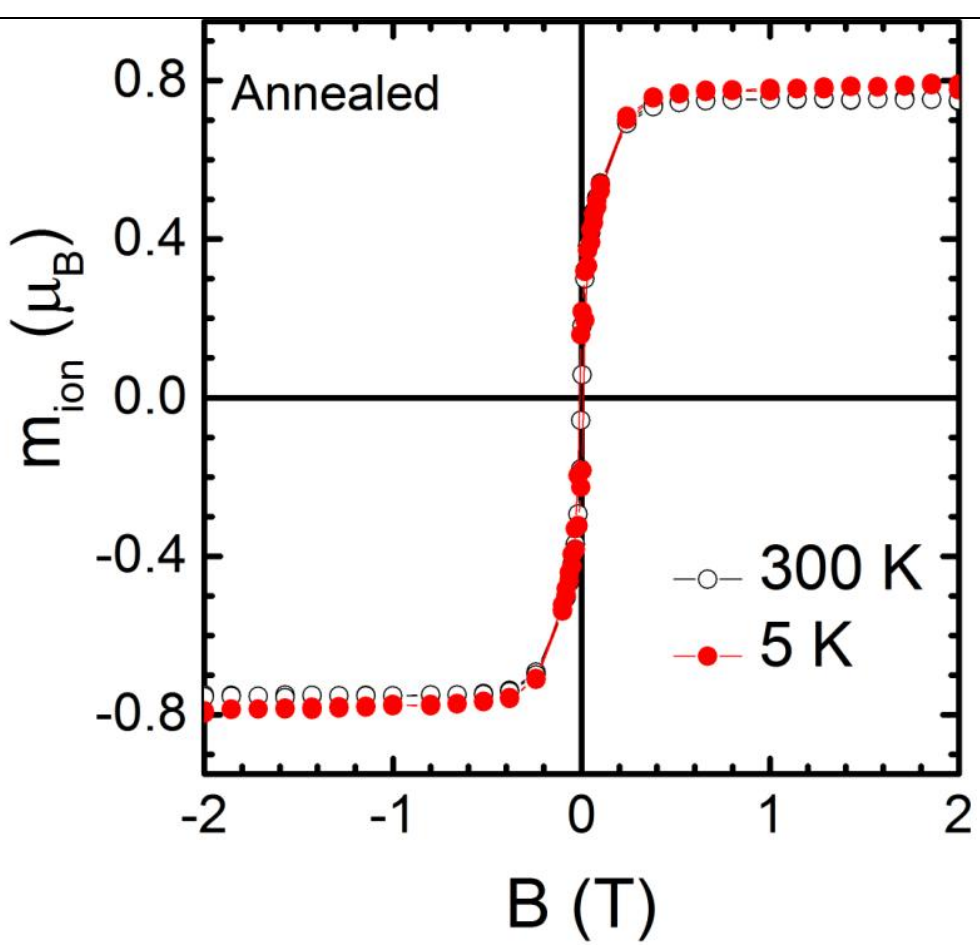

Figure 6.2.7. The moment per implanted ion against the magnetic field for the sample that was electron-beam annealed at $1000 \mathrm{C}$ for 1 hour at $5 \mathrm{~K}$ (filled circles) and $300 \mathrm{~K}$ (open circles). 
$m_{\text {ion,s }}$ monotonically decreases with temperature as can be seen in Figure 6.2.8a. However, it can clearly not be fitted using Equation 2.11 and with the bulk $\beta=3 / 2$. For this reason the data were fitted using Equation 6.1.3 and $\beta=3 / 2$ where we find that $m_{\text {core }}(0)=(0.751 \pm 0.005) \mu_{\mathrm{B}}, \quad B=(7 \pm 1) \times 10^{-5} \quad \mathrm{~K}^{3 / 2}$, $m_{\text {shell }}(0)=(0.028 \pm 0.004) \mu_{\mathrm{B}}$, and $T_{\mathrm{f}}=(68 \pm 25) \mathrm{K} . B$ and $T_{\mathrm{f}}$ are the same as those found for $x=0.82$ within the experimental uncertainty. The asymmetric nanoparticle shapes for $x=0.55$ make it difficult to estimate the thickness of the moment disordered region. However, $m_{\text {shell }}(0) /\left[m_{\text {core }}(0)+m_{\text {shell }}(0)\right]$ is small and it is 0.036 , which implies that the spin-disordered region is thin.

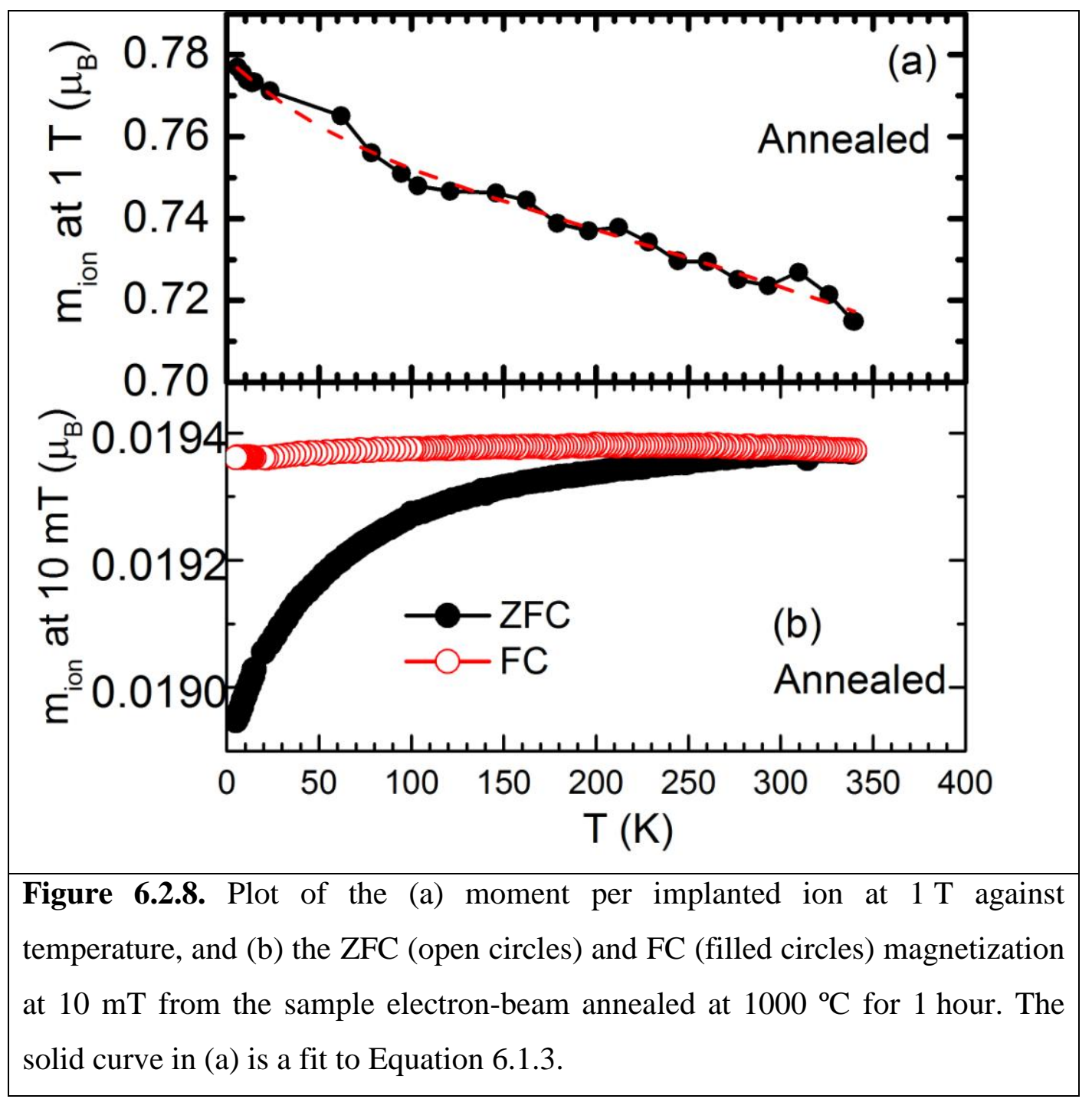


There is no evidence for superparamagnetic behaviour for the EBA sample as can be seen from the ZFC and FC magnetization in Figure 6.2.8. Hysteresis is observed up to the highest measured temperature indicating that any blocking temperature would need to be above $340 \mathrm{~K}$. This is consistent with the particle sizes estimated from the TEM data where the nanoparticles are too large for superparamagnetism to be observed.

$M / H$ against $B$ is plotted in Figure 6.2.9 for the EBA sample and the asimplanted sample. $M / H$ was obtained from the measured moment using Equation 6.1.5. The as-implanted film has a maximum $\chi$ value of 5 at low fields and the annealed film shows an increased value of $\sim 15$. The annealed $\chi$ is comparable to that found for the $x=0.82$ sample. As noted earlier, these values are lower than those seen in the bulk, which is due to the effect of demagnetization.

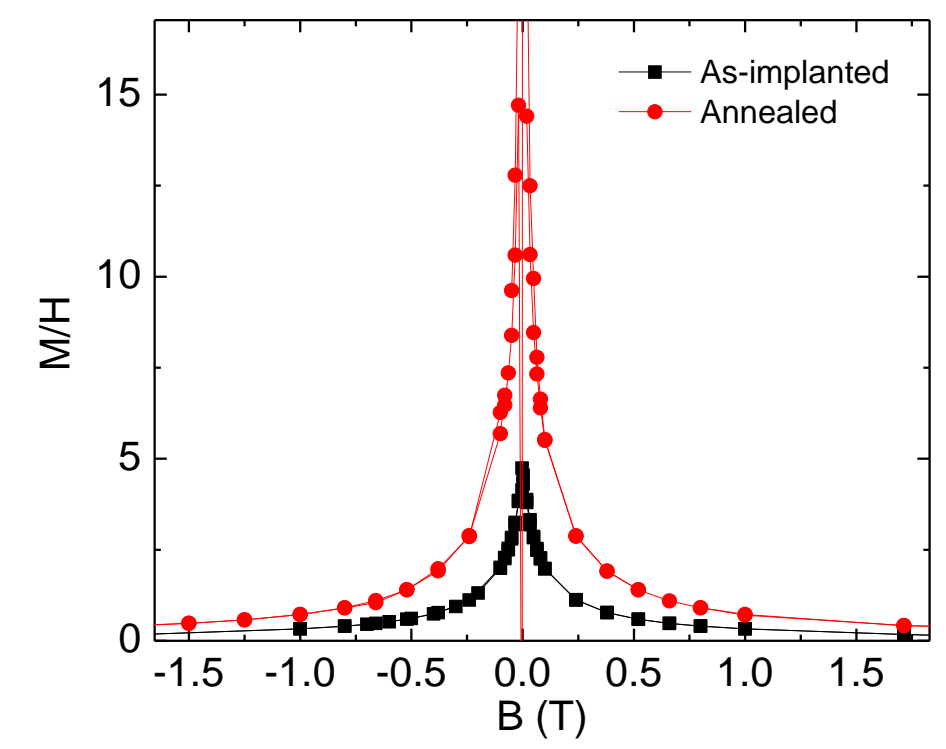

Figure 6.2.9. Plot of $\mathrm{M} / \mathrm{H}$ for the as-implanted sample (filled squares) and the sample that was EBA at $1000{ }^{\circ} \mathrm{C}$ for 1 hour (filled circles). 


\subsubsection{Conclusions}

In conclusion, low energy dual implantation of $\mathrm{Ni}$ and $\mathrm{Fe}$ into $\mathrm{SiO}_{2}$ with a $\mathrm{Ni}$ fraction of 0.45 has resulted in the formation of superparamagnetic magnetic nanoparticles and $\mathrm{EBA}$ at $1000^{\circ} \mathrm{C}$ for 1 hour led to $\mathrm{Ni}_{x} \mathrm{Fe}_{1-x}$ magnetic nanoparticles were some nanoparticles had a $\mathrm{Fe}_{1-y} \mathrm{Si}_{y} \mathrm{O}_{z}$ shell. The as-implanted sample had particle sizes ranging from $2 \mathrm{~nm}$ to $5 \mathrm{~nm}$ where the average size was 4 $\mathrm{nm}$, which is similar to that in a previous study with a similar fluence and $x=0.82$. The blocking temperature $(60 \mathrm{~K})$ is significantly higher than that reported for $x=0.82\left(T_{\mathrm{B}}=13 \mathrm{~K}\right)$, which may be due to a higher magnetocrystalline anisotropy or nanoparticles with different domain wall pinning energies. The saturation moment per implanted ion can be fitted with a Bloch exponent of $3 / 2$ and spin-glass shell component that had a freezing temperature of $17 \mathrm{~K}$ and an estimated thickness of $0.1 \mathrm{~nm}$. This can be compared with a Bloch exponent of 0.38 for the $x=0.82$ sample. This shows that the $x=0.45$ nanoparticles magnetically behave more like isolated nanoparticles with spin-waves localised in the nanoparticles. Similar to the $x=0.82$ sample we find that the saturation moment at low temperature is significantly less than the bulk value, which is likely to be due to unordered moments as well as antiferromagnetic phases.

The EBA sample had $\mathrm{Ni}_{x} \mathrm{Fe}_{1-x}$ nanoparticles with widths ranging from $\sim 72 \mathrm{~nm}$ to $\sim 140 \mathrm{~nm}$, depths ranging from $60 \mathrm{~nm}$ to $170 \mathrm{~nm}$, and there are very few smaller particles with sizes of $\sim 15 \mathrm{~nm}$. The susceptibility is enhanced after EBA. The size range for the larger nanoparticles is comparable to that found for $x=0.82$. However, the nanoparticles for $x=0.45$ are more asymmetrical and some samples contain $\mathrm{Ni}_{x} \mathrm{Fe}_{1-x}$ cores with $\mathrm{Fe}_{1-y} \mathrm{Si}_{y} \mathrm{O}_{z}$ shells that was not seen for $x=0.82$. It was previously not possible to determine if $\mathrm{Ni}_{x} \mathrm{Fe}_{1-x}$ nanoparticles rather than $\mathrm{Fe}$ and $\mathrm{Ni}$ nanoparticles were formed for $x=0.82$ due to the sizable bimodal particle size distribution with $\sim 10 \mathrm{~nm}$ nanoparticles extending to $60 \mathrm{~nm}$ to $120 \mathrm{~nm}$ into the film that is not clearly evident for $x=0.45$. The appearance of excess Fe for $x=0.45$ and 
not for $x=0.82$ may be related to the nanoparticle growth mechanism where $\mathrm{Ni}_{x} \mathrm{Fe}_{1-x}$ nanoparticles in the as-implanted film with $x$ close to 0.3 have the lowest Gibbs free energy of formation and act as nucleation centres. This scenario would lead to $\mathrm{Ni}_{x_{2}} \mathrm{Fe}_{1-x_{2}}$ nanoparticles with $x_{2}$ close to 0.7 and excess Fe. This is supported by the magnetic data where the saturation moment per implanted ion is significantly less than that expected from the bulk and we estimate that $x_{2}=0.71$. The ferromagnetic nanoparticles for $x=0.45$ have a saturation moment that follows Bloch 3/2 law found in bulk $\mathrm{Ni}_{x} \mathrm{Fe}_{1-x}$ as well as the $x=0.82$ sample where there is a spin-glass from the uncompensated surface moments and the spin freezing temperature is comparable for $x=0.45$ and $x=0.82$. Our results show that there are some similarities as well as considerable differences for $\mathrm{Ni}$ and $\mathrm{Fe}$ dual implanted and EBA samples with $x=0.45$ and $x=0.82$. 


\subsubsection{High fluence iron-nickel nanoparticles embedded in silicon dioxide}

The previous two sections reported the results from dual $\mathrm{Ni}$ and $\mathrm{Fe}$ implantation into $\mathrm{SiO}_{2}$ with the same $\mathrm{Ni}$ fluence but different $\mathrm{Fe}$ fluences and with $\mathrm{Ni}$ concentrations being close to the commonly used permalloy 78 and permalloy 45. In this section the effect of a higher Ni fluence on the structural and magnetic properties is explored where the $\mathrm{Ni}$ concentration is 0.53 , which is comparable to that used in section 6.2.1.

\subsubsection{Experimental details}

$\mathrm{Ni}_{x} \mathrm{Fe}_{1-x}$ nanoclusters embedded in silicon dioxide were made using the same methods described in the previous sections. The implanted nickel fluence was double of that used in sections 6.1 and 6.2 .1 and it was $4 \times 10^{16} \mathrm{at} . / \mathrm{cm}^{2}$. The ${ }^{56} \mathrm{Fe}+$ was implanted into the nickel-implanted samples with the fluence of $3.5 \times 10^{16}$ at. $/ \mathrm{cm}^{2}$. The nickel to iron fluence ratio is $53: 47$ and it is comparable to that used in section 6.2.1. D-TRIM simulations were used for depth and concentration profiling at the implantation energy. RBS measurements were performed using a $2 \mathrm{MeV} \mathrm{He}$ beam and current of $\sim 20 \mathrm{nA}$. The fluences were obtained by fitting using RUMP software.

\subsubsection{D-TRIM simulations}

Figure 6.2.2.1 shows the D-TRIM simulation for a high fluence sample of $\mathrm{Ni}$ and Fe sequentially implanted. The nickel fluence is $4 \times 10^{16}$ at. $/ \mathrm{cm}^{2}$ and the $\mathrm{Fe}$ fluence is $3.5 \times 10^{16}$ at. $/ \mathrm{cm}^{2}$. The simulated average depth of the nickel and iron are comparable. The maximum depth of both is $\sim 35 \mathrm{~nm}$. The average estimated depth of both is $\sim 12.5 \mathrm{~nm}$ with maximum concentrations of $32 \%$ and $28 \%$ for $\mathrm{Ni}$ and $\mathrm{Fe}$, respectively. The average straggling depth of either element is $\sim 12 \mathrm{~nm}$. A concentration of $25 \% \mathrm{Fe}$ and $33 \% \mathrm{Ni}$ is at the surface. The $\mathrm{Fe}$ concentration ratio is also plotted in Figure 6.2.2.1. The Fe concentration increases slightly up to 
$\sim 20 \mathrm{~nm}$ into the surface caused by sputtering of ions and it is $46 \%$ at the peak $\mathrm{Ni}$ and Fe concentration. It then increases up to a maximum of $62 \%$ at $28 \mathrm{~nm}$.

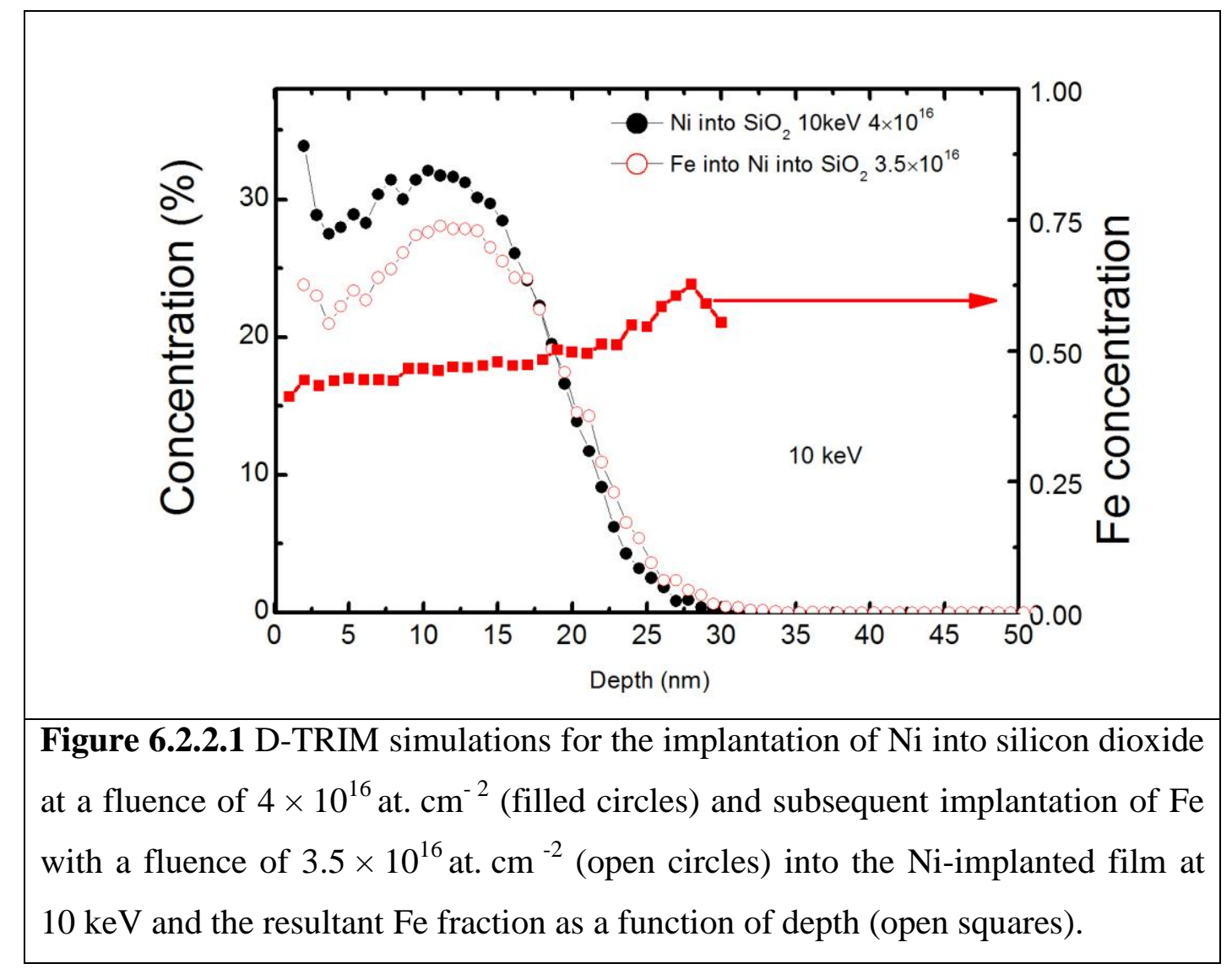

\subsubsection{Rutherford backscattering analysis}

Figure 6.2.2.2 shows the Rutherford backscattering data for the high fluence film. As noted in the RBS data in the previous sections the silicon edge, the silicon dioxide on silicon interface edge, and the oxygen edge can be clearly seen in the data. The peak at $\sim 1.5 \mathrm{MeV}$ is due to $\mathrm{Ni}$ and $\mathrm{Fe}$ in the film where the $\mathrm{Ni}$ and Fe edges cannot be clearly resolved. The RBS data were fitted using RUMP software to obtain the total $\mathrm{Ni}$ and $\mathrm{Fe}$ fluences. The resultant $\mathrm{Ni}$ and $\mathrm{Fe}$ fluences were $3.86 \times 10^{16}$ at. $/ \mathrm{cm}^{2}$ and $3.48 \times 10^{16}$ at. $/ \mathrm{cm}^{2}$ for the as-implanted film and assuming that the $\mathrm{Ni}$ to $\mathrm{Fe}$ ratio is the same as that used during the implantation process. 


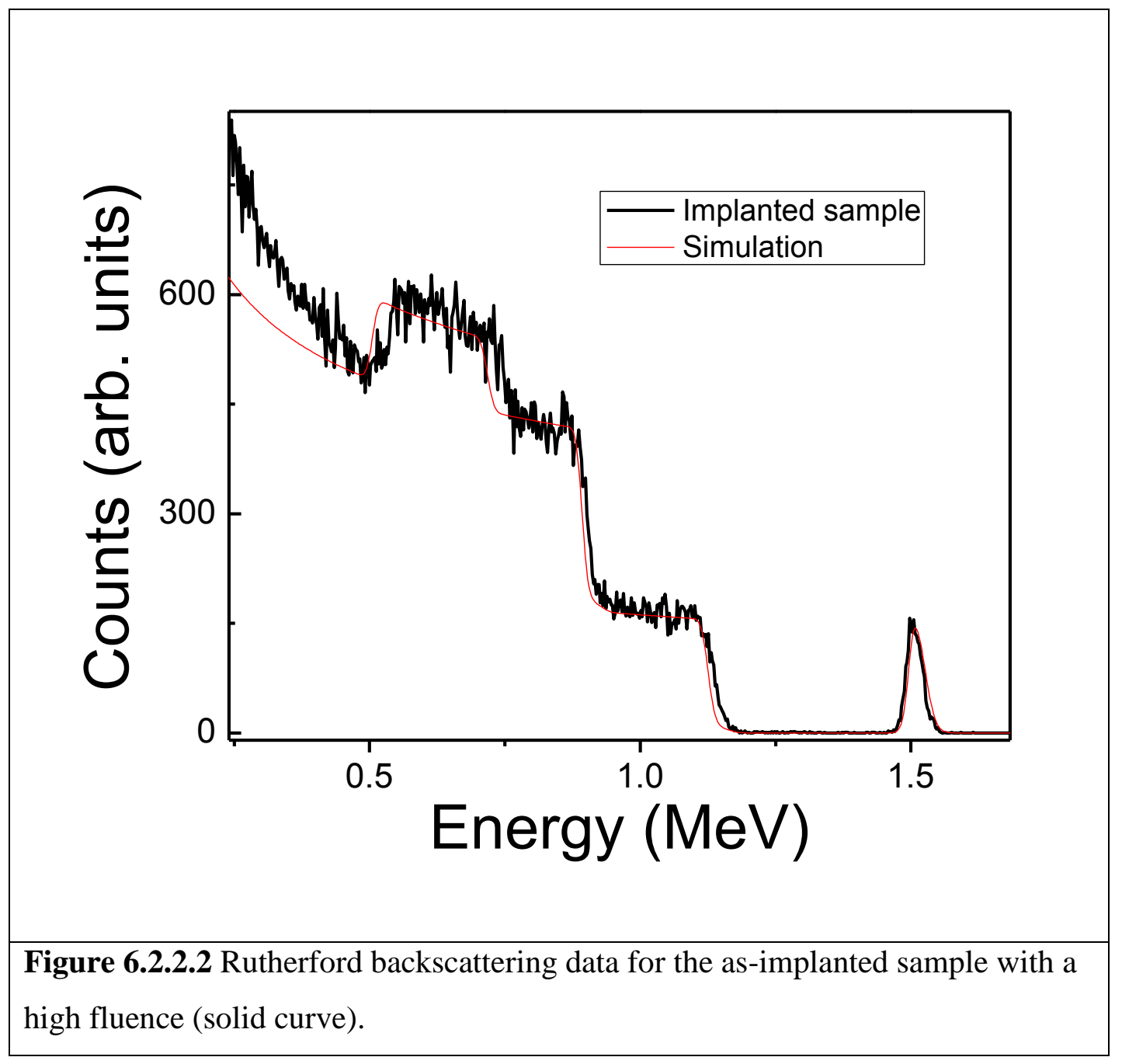

\subsubsection{Magnetization measurements}

Magnetization measurements on the higher fluence film are shown in Figure 6.2.2.3. The moment per implanted ion $\left(\mathrm{m}_{\mathrm{ion}}\right)$ at $300 \mathrm{~K}$ and $5 \mathrm{~K}$ are plotted against the applied field with a maximum of $2 \mathrm{~T}$. Measurements above $2 \mathrm{~T}$ were omitted due to the noise. $m_{\text {ion }}$ was calculated from the measured moment after $\mathrm{Si}$ subtraction using Equation 6.11. The moment per implanted ion is $1.5 \mu_{\mathrm{B}}$. This is slightly less than that reported for $\mathrm{x}=0.50$ where the saturation moment per ion was $1.7 \mu_{\mathrm{B}}$ (Crangle and Goodman, 1971). There is no indication of a lack of saturation at the high fields. This indicates less spin disorder when compared with 
the lower fluence sample plotted in Figure 6.2.4 in section 6.2.1. The saturation moment per ion at $300 \mathrm{~K}$ is lower at $5 \mathrm{~K}$ due to spin waves.

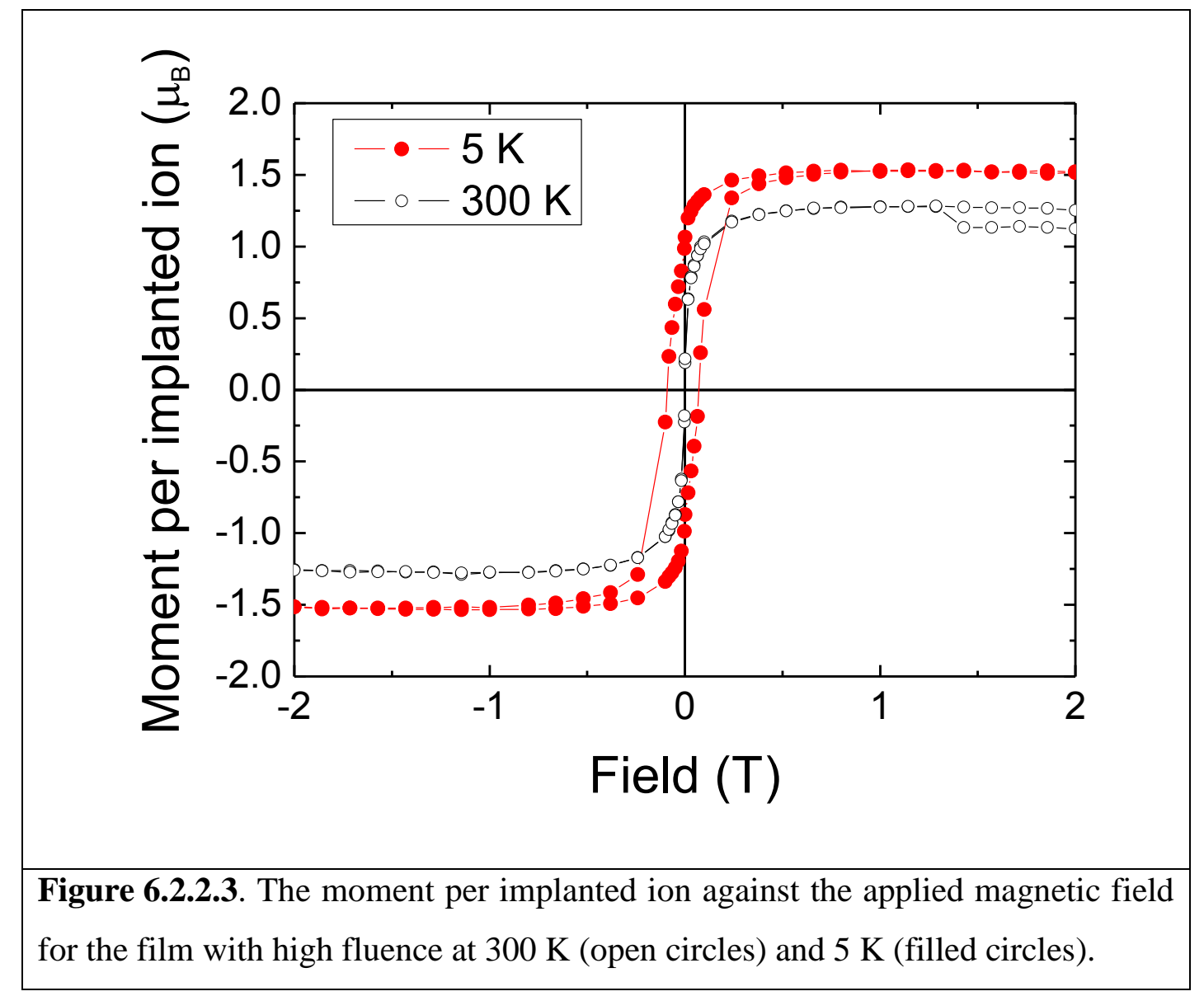

In Figure 6.2.2.4, the ZFC and FC curves for high fluence film is plotted for an applied magnetic field of $10 \mathrm{mT}$. The ZFC-FC curves show typical superparamagnetic behaviour. The blocking temperature is $170 \mathrm{~K}$. This is larger than the $60 \mathrm{~K}$ found for the lower fluence sample and indicates that the average particle size is larger. Using Eq. 2.12 it is possible to estimate that the average particle size has increased by a factor of $\sim 1.4$. 


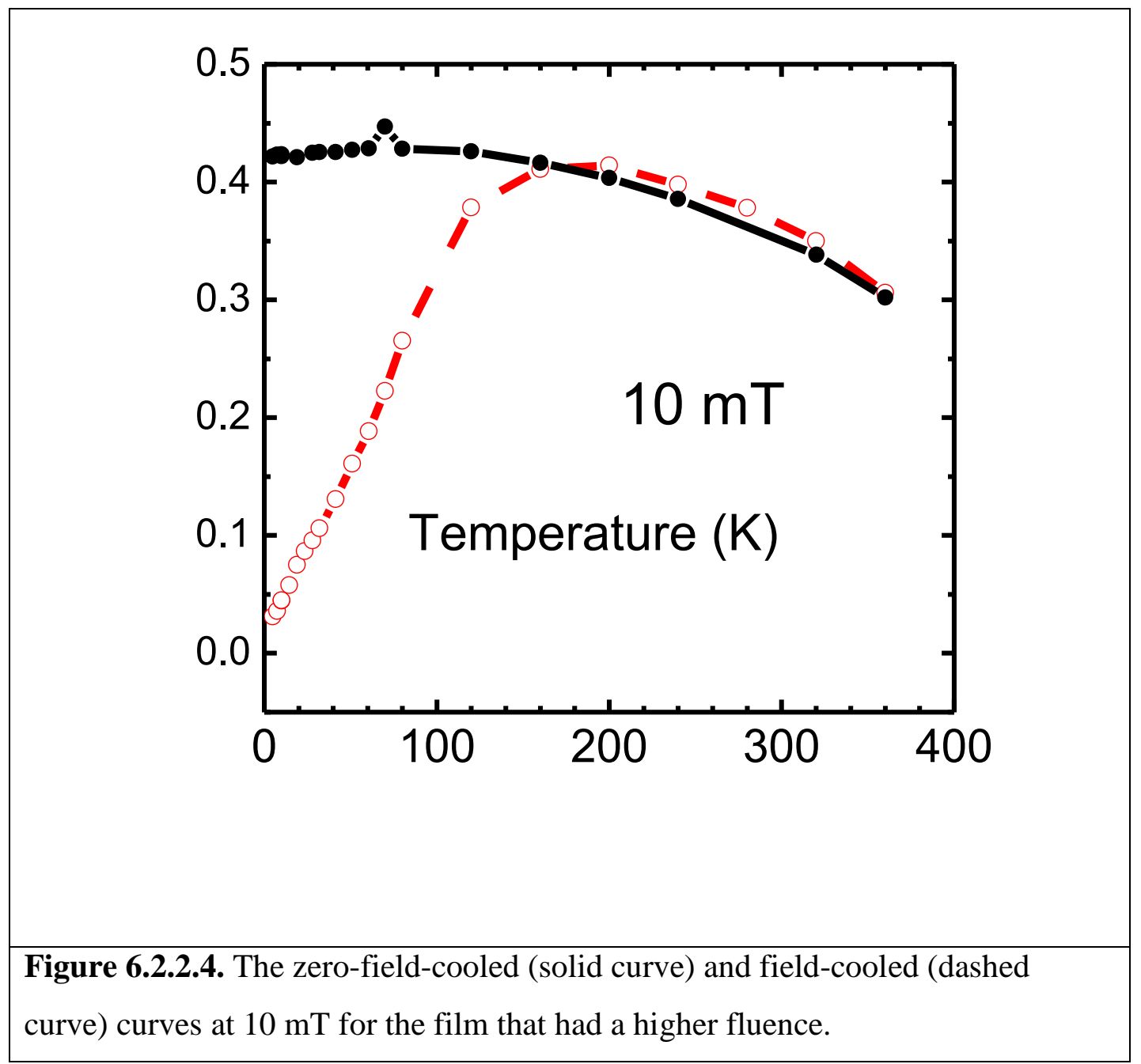

Figure 6.2.2.5 shows the susceptibility of the high fluence film plotted against the applied field. The susceptibility was obtained Eq. 6.1.5 and it reached 90 at $300 \mathrm{~K}$. This is a factor of 7.5 greater than that seen for as-implanted and 5.6 times greater than the annealed films with a lower fluence. The susceptibility is much less than expected for annealed bulk permalloy where values are greater than 10000. This is due to demagnetization effects. The higher susceptibilities may be due to different domain wall pinning. There is no evidence for two magnetic phases that was seen for the lower fluence films. 


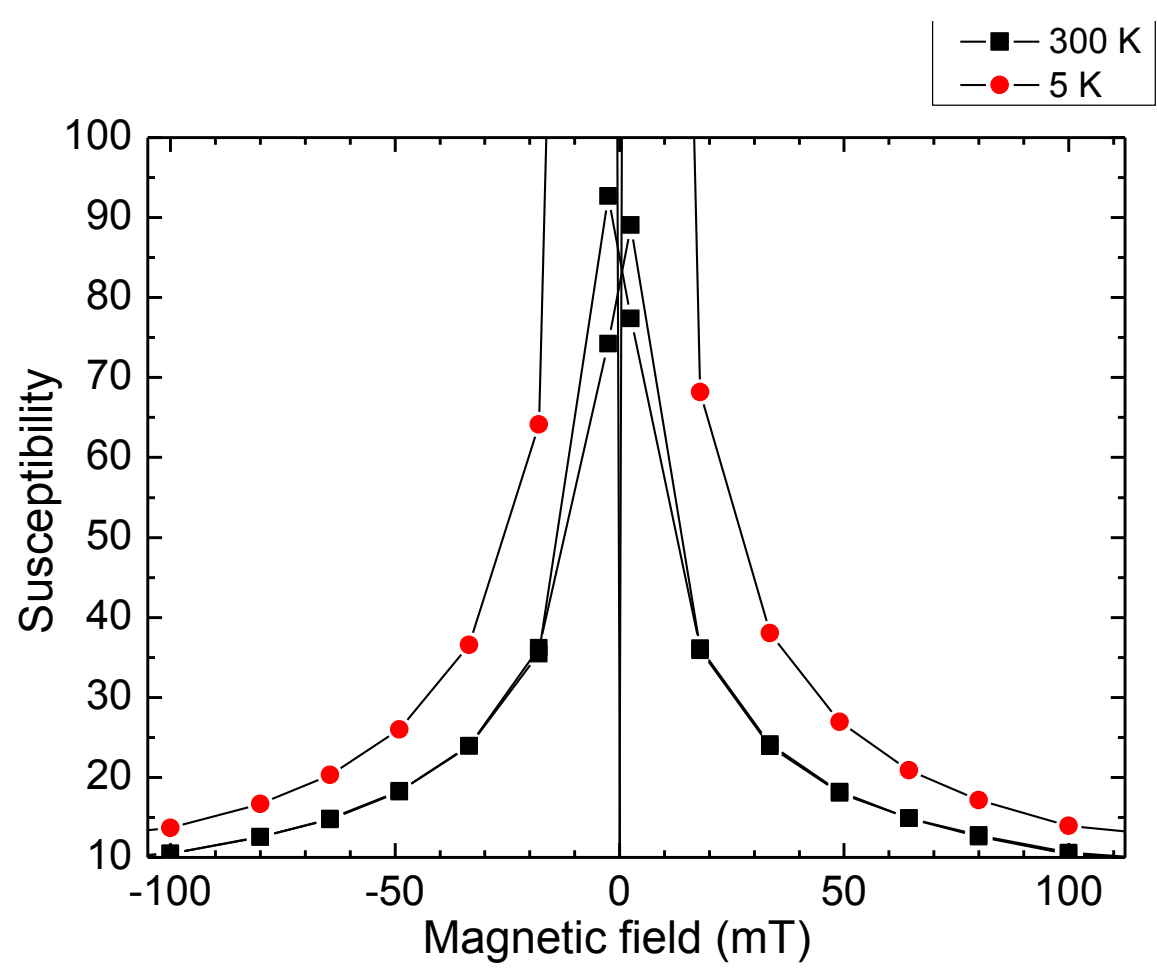

Figure 6.2.2.5. The susceptibilities at room temperature for the high fluence sample at $300 \mathrm{~K}$ (black closed squares) and $5 \mathrm{~K}$ (red closed circles).

The saturation moment per ion is plotted against temperature as shown in Figure 6.2.2.6 for the as-implanted film. It is apparent that the data cannot be fitted to Eq. 2.11. In can be seen in Figure 6.2.2.6 that the saturation moment per ion can be fitted to Eq. 6.1 .3 with $\beta=3 / 2$ and indicates that there is a spindisordered region in the nanoparticle surface region. The fitted values are $m_{\text {ion,bulk }}(0)=1.479 \mu_{\mathrm{B}}, \quad B=(2.48 \pm 0.04) \times 10^{-5} \mathrm{~K}^{-1.5}, \quad m_{\text {ion,surface }}(0)=0.0608 \quad \mu_{\mathrm{B}}, \quad$ and $T_{\mathrm{f}}=15.594 \pm 1 \mathrm{~K}$. It can be seen that $B$ and $T_{\mathrm{f}}$ are similar to that found in for the lower fluence as-implanted sample, which might be expected given that the average sizes are comparable. 
The thickness of the spin-disordered shell was estimated using Eq. 6.1.4 and it was found to be $t_{\text {shell }}=0.0195 r$. This is smaller than that found for the lower fluence sample and suggests lower spin-disorder in the shell.

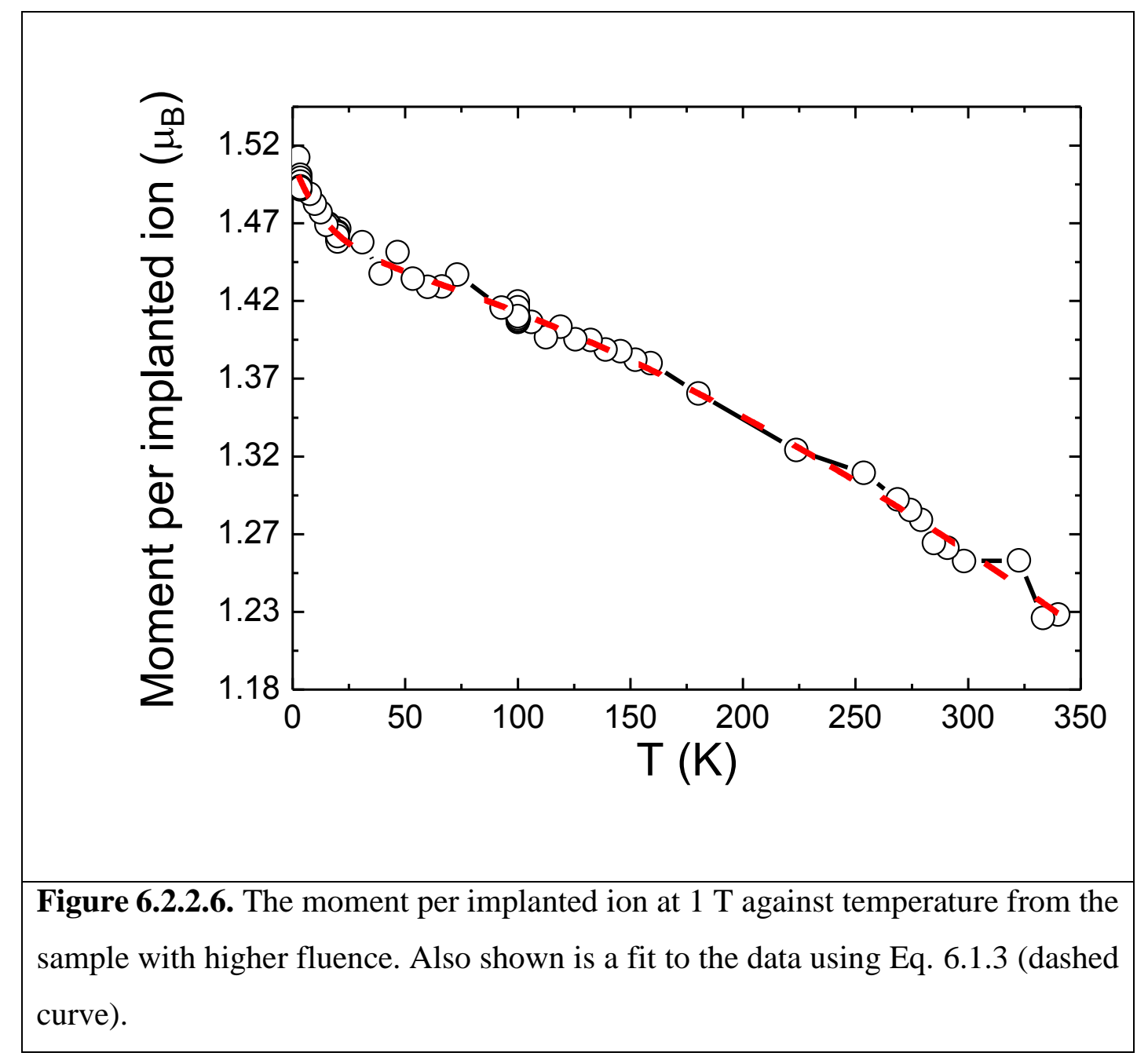

\subsubsection{Conclusions}

In conclusion, superparamagnetic nanoclusters of $\mathrm{Ni}_{x} \mathrm{Fe}_{1-x}$ alloy have been synthesized using ion implantation. The Ni concentration increases slightly up to $20 \mathrm{~nm}$ and it is 0.53 at the peak concentration. The saturation moment per ion at $5 \mathrm{~K}$ is $1.5 \mu_{\mathrm{B}}$, which is slightly less than the expected value of $1.7 \mu_{\mathrm{B}}$ for $\mathrm{x}=0.5$. This is possibly due to the presence of antiferromagnetic phase or spins that are 
not magnetically ordered. The value is much higher than that of the $\mathrm{x}=0.82$ or $\mathrm{x}=0.45$, indicating that there is less spin disorder and more of the spins have magnetically ordered. The zero-field cooled and field cooled measurements show the presence of superparamagnetic $\mathrm{Ni}_{x} \mathrm{Fe}_{1-x}$ and the blocking temperature is $170 \mathrm{~K}$. This is higher than that of the lower fluence samples and indicates larger average nanoparticle sizes. The susceptibility reaches 90 , which is higher than that of the lower fluence samples. The temperature dependence of the saturation moment per ion can be modelled in terms of a $\beta=3 / 2$ and a surface disorder term that had a spin freezing temperature of $16 \mathrm{~K}$. 


\subsection{Summary}

Magnetic $\mathrm{Ni}_{x} \mathrm{Fe}_{1-x}$ nanoparticles were made for the first time by low energy ion implantation into silicon dioxide films with two different $\mathrm{Ni}$ and $\mathrm{Fe}$ fractions that are close to those found in the commonly used bulk permalloy 78 and permalloy 45 .

As-implanted films with $\mathrm{x}=0.82$ and $\mathrm{x}=0.45$ and with the same fluence showed an implanted film layer with $\mathrm{Ni}_{x} \mathrm{Fe}_{1-x}$ nanoparticles with similar sizes and an average diameter of $4 \mathrm{~nm}$. The nanoparticles were superparamagnetic and the resultant magnetocrystalline anisotropies were enhanced over that seen in the bulk, which is known to occur in small magnetic nanoparticles. It was largest for $\mathrm{x}=0.45$. The saturation magnetic moments per ion were significantly less than that found in the bulk, which is likely to be due to moments that do not magnetically order and antiferromagnetic $\mathrm{Ni}_{\mathrm{x}} \mathrm{Fe}_{1-\mathrm{x}} \mathrm{Si}_{\mathrm{y}} \mathrm{O}_{\mathrm{z}}$ phases. The temperature dependence of the saturation moment per ion was different for $\mathrm{x}=0.82$ and $\mathrm{x}=0.45$. It was found the temperature exponent was 0.38 for $\mathrm{x}=0.82$, which is likely to be due magnon propagation between nanoparticles that is not seen in isolated nanoparticles as well as the effect of disordered surface spins. This can be contrasted with the $\mathrm{x}=0.45$ sample where the exponent is $3 / 2$ and this is closer to that seen in isolated nanoparticles as well as in bulk $\mathrm{Ni}_{x} \mathrm{Fe}_{1-x}$. The prefactor, $\mathrm{B}$, is larger than that found in the bulk, which is known to occur in other magnetic nanoparticles systems. The nanoparticles in the $\mathrm{x}=0.45$ sample have a spin-disordered shell, which is likely to be due to uncompensated surface spins. The data were modelled with a surface spin-disordered freezing temperature of $17 \mathrm{~K}$. The susceptibility was highest for $\mathrm{x}=0.82$ and it reached a value of 12 . This is significantly less than that seen in the bulk, which is likely to be due to demagnetization effects as well as domain wall pinning.

A sample was made with a higher fluence but with $x=0.53$ that was close to that for the $\mathrm{x}=0.45$ sample. Unlike the lower fluence sample, the saturation 
moment per ion was only slightly less than that found in the bulk and indicating that most of the implanted ions magnetically ordered. Superparamagnetism was also observed but the blocking temperature was higher indicating larger nanoparticles by a factor of $\sim 1.4$. The temperature dependence of the saturation moment per ion could be fitted with the bulk temperature exponent of $3 / 2$ and well as a surface spin disordered component. The prefactor, B, and the spinfreezing temperature were comparable to that found for the lower fluence sample. This might be expected based on the comparable nanoparticles sizes. The susceptibility was much larger and it reached 90 . The larger value may be due to a difference in the magnetic domain wall pinning energies.

Electron beam annealing of the $\mathrm{x}=0.82$ and $\mathrm{x}=0.45$ samples resulted in different nanoparticles morphologies. EBA the $\mathrm{x}=0.82$ sample resulted in a bimodal particle size distribution with large and nearly symmetric nanoparticles in the near surface region with sizes from $40 \mathrm{~nm}$ to $75 \mathrm{~nm}$ and an average diameter of $44 \mathrm{~nm}$. Small 10nm diameter nanoparticles extended into the film from $60 \mathrm{~nm}$ to $120 \mathrm{~nm}$ and some nanoparticles were seen at the $\mathrm{SiO}_{2} / \mathrm{Si}$ interface. There was no bimodal particle size distribution for the $\mathrm{x}=0.45$ sample and asymmetric near surface nanoparticles were observed with sizes of $\sim 2 \mathrm{~nm}$ to $140 \mathrm{~nm}$ along with a few small nanoparticles. Some nanoparticles were found that have $\mathrm{Ni}_{x} \mathrm{Fe}_{1-x}$ cores and $\mathrm{FeSi}_{\mathrm{y}} \mathrm{O}_{\mathrm{x}}$ shells. The different nanoparticles morphologies is likely to be due to $\mathrm{Ni}_{x} \mathrm{Fe}_{1-x}$ nucleation sites with different values of $\mathrm{x}$ and the different initial concentration profiles. The saturation magnetization for the $\mathrm{x}=0.82$ sample was close to that expected for the bulk while it was significantly less than the expected value for $x=0.45$. The lower value for $x=0.45$ is likely to be due to the nanoparticle growth mechanism as well as excess Fe in the nanoparticle shell that may form an antiferromagnetic phase. The temperature dependence of the saturation moment per ion could be fitted to the bulk value of $3 / 2$ and there was also evidence of nanoparticle surface spin-disorder. The spin freezing temperatures were higher than those seen in the as-implanted samples but they 
were the same with errors. The prefactor, B, was a factor of two larger than the bulk value for the P78 sample and less than that seen in the as-implanted samples, which suggests that it depends on the nanoparticle size. The $\mathrm{x}=0.53$ sample has a $\mathrm{B}$ that is a factor of 10 higher and it is $\sim 3$ times higher than that found for the asimplanted samples. This may be related to the appearance of an Fe-rich shell and the effect of magnon propagation within part of this shell. The susceptibility for both samples was comparable and $\sim 16$, which was slightly larger than that seen in the as-implanted samples.

\section{References}

Amekura, H., Kitazawa, H., Umeda, N., Takeda, Y., Kishimoto, N., 2004b. Nickel nanoparticles in silica glass fabricated by $60 \mathrm{keV}$ negative-ion implantation. Nuclear Instrument and Methods in Physics Research Section B Beam Interactions with Materials and Atoms 222, 114-122. doi:10.1016/j.nimb.2004.01.214

Anderson, T.S., Magruder, R.H., Kinser, D.L., Zuhr, R.A., Thomas, D.K., 1997. Formation and optical properties of metal nanoclusters formed by sequential implantation of $\mathrm{Cd}$ and $\mathrm{Ag}$ in silica. Nuclear Instrument and Methods in Physics Research Section B Beam Interactions with Materials and Atoms 124, 40-46.

Aquino, R., Depeyrot, J., Sousa, M.H., Tourinho, F.A., Dubois, E., Perzynski, R., 2005. Magnetization temperature dependence and freezing of surface spins in magnetic fluids based on ferrite nanoparticles. Physical Review B 72. doi:10.1103/PhysRevB.72.184435

Balakirev, N., Zhikharev, V., Gumarov, G., 2014. The formation of magnetic silicide $\mathrm{Fe}_{3} \mathrm{Si}$ clusters during ion implantation. Nuclear Instrument and Methods in Physics Research Section B Beam Interactions with Materials and Atoms 326, 61-64. doi:10.1016/j.nimb.2013.09.032

Ban, I., Drofenik, M., Makovec, D., 2006. The synthesis of iron-nickel alloy nanoparticles using a reverse micelle technique. Journal of Magnetism and Magnetic Materials 307, 250-256. doi:10.1016/j.jmmm.2006.04.010

Biersack, J.P., Berg, S., Nender, C., 1991. T-DYN Monte Carlo simulations applied to ion assisted thin film processes. Nuclear Instrument and Methods in Physics Research Section B Beam Interactions with Materials and Atoms 59, 21-27.

Bloch, F., 1930. Zur theorie des ferromagnetismus. Z. Für Phys. 61, 206-219.

Bozorth, R.M., 1951. Ferromagnetism. 
Bozorth, R.M., Walker, J.G., 1953. Magnetic crystal anisotropy and magnetostriction of iron-nickel alloys. Physical Review 89, 624.

Chase, M.W., 1998. NIST-JANAF Themochemical Tables, Fourth Edition. Journal of Physical and Chemical Reference Data, Monograph 9 1-1951.

Chudnovsky, E.M., Saslow, W.M., Serota, R.A., 1986. Ordering in ferromagnets with random anisotropy. Physical Review B 33, 251.

Cojocaru, S., Naddeo, A., Citro, R., 2014. Modification of the Bloch law in ferromagnetic nanostructures. EPL Europhysics Letters 106, 17001. doi:10.1209/0295-5075/106/17001

Crangle, J., Goodman, G.M., 1971. The magnetization of pure iron and nickel, in: Proceedings of the Royal Society of London A: Mathematical, Physical and Engineering Sciences. The Royal Society, pp. 477-491.

Cullity, B.D., Graham, C.D., 2011. Introduction to magnetic materials. John Wiley \& Sons.

Cushing, B.L., Golub, V., O’Connor, C.J., 2004. Synthesis and magnetic properties of Au-coated amorphous Fe20Ni80 nanoparticles. Journal of Physics and Chemistry of Solids 65, 825-829. doi:10.1016/j.jpcs.2003.11.027

Demortière, A., Panissod, P., Pichon, B.P., Pourroy, G., Guillon, D., Donnio, B., Bégin-Colin, S., 2011. Size-dependent properties of magnetic iron oxide nanocrystals. Nanoscale 3, 225-232. doi:10.1039/C0NR00521E

Gangopadhyay, S., Hadjipanayis, G.C., Dale, B., Sorensen, C.M., Klabunde, K.J., Papaefthymiou, V., Kostikas, A., 1992. Magnetic properties of ultrafine iron particles. Physical Review B 45, 9778.

Ghoshtagore, R.N., 1969. Diffusion of nickel in amorphous silicon dioxide and silicon nitride films. Journal of Applied Physics 40, 4374. doi:10.1063/1.1657201

Gianuzzi, 1999. Focused ion beam milling for site specific scanning and transmission electron microscopy of materials. Micron 30, 197.

Hendriksen, P.V., Linderoth, S., Lindgla ard, P.-A., 1993. Finite-size modifications of the magnetic properties of clusters. Physical Review B 48, 7259 .

Kennedy, J., Leveneur, J., Markwitz, A., 2012a. High temperature annealing effects on low energy iron implanted $\mathrm{SiO}_{2}$. Nuclear Instrument and Methods in Physics Research Section B Beam Interactions with Materials and Atoms 273, 182-185. doi:10.1016/j.nimb.2011.07.070

Kennedy, J., Leveneur, J., Takeda, Y., Williams, G.V.M., Kupke, S., Mitchell, D.R.G., Markwitz, A., Metson, J.B., 2012b. Evolution of the structure and magneto-optical properties of ion beam synthesized iron nanoclusters. Journal of Material Science 47, 1127-1134. doi:10.1007/s10853-0115849-1

Kennedy, J., Leveneur, J., Williams, G.V.M., Mitchell, D.R.G., Markwitz, A., 2011. Fabrication of surface magnetic nanoclusters using low energy ion 
implantation and electron beam annealing. Nanotechnology 22, 115602. doi:10.1088/0957-4484/22/11/115602

Kubaschewski, O., von Goldbeck, O., 1949. The thermodynamics of the ironnickel alloys. Transactions of the Faraday Society 45, 948-960.

Lakshantha, W.J., Dhoubhadel, M.S., Reinert, T., McDaniel, F.D., Rout, B., 2015. Investigation of various phases of $\mathrm{Fe}-\mathrm{Si}$ structures formed in $\mathrm{Si}$ by low energy Fe ion implantation. Nuclear Instrument and Methods in Physics Research Section B Beam Interactions with Materials and Atoms 365, 114-119. doi:10.1016/j.nimb.2015.07.037

Leveneur, J., Kennedy, J., Williams, G.V.M., Metson, J., Markwitz, A., 2011. Large room temperature magnetoresistance in ion beam synthesized surface Fe nanoclusters on $\mathrm{SiO}_{2}$. Applied Physics Letters 98, 053111. doi:10.1063/1.3553274

Leveneur, J., Kennedy, J., Williams, G.V.M., Sasase, M., Metson, J.B., Markwitz, A., 2014a. Structural and chemical changes during the growth of $\mathrm{Fe}$ nanoparticles in $\mathrm{SiO}_{2}$ under low energy ion implantation. International Journal of Nanotechnology 11, 466-476.

Leveneur, J., Kennedy, J., Williams, G.V.M., Sasase, M., Metson, J.B., Markwitz, A., 2014b. Structural and chemical changes during the growth of $\mathrm{Fe}$ nanoparticles in $\mathrm{SiO}_{2}$ under low energy ion implantation. International Journal of Nanotechnology 11, 466-476.

Leveneur, J., Waterhouse, G.I.N., Kennedy, J., Metson, J.B., Mitchell, D.R.G., 2011. Nucleation and Growth of Fe Nanoparticles in $\mathrm{SiO}_{2}$ : A TEM, XPS, and Fe L-Edge XANES Investigation. Journal of Physical Chemistry C 115, 20978-20985. doi:10.1021/jp206357c

Li, X., Chiba, A., Takahashi, S., 1997. Preparation and magnetic properties of ultrafine particles of Fe-Ni alloys. Journal of Magnetism and Magnetic Materials 170, 339-345.

Li, X., Jin, S., Dong, C., Zhang, Z., Gong, Z., Ma, T., 1997. Transmission electron microscopic studies of ternary FeNi-silicide layers prepared by metal vapour vacuum arc ion implantation. Thin Solid Films 304, 196-200.

Li, X.N., Dong, C., Jin, S., Ma, T.C., Zhang, Q.Y., 1998. Ion beam synthesis of $\mathrm{Ni}-\mathrm{Fe}-\mathrm{Si}$ layer by TEM. Surface Coating Technology 103, 231-234.

Maaz, K., Mumtaz, A., Hasanain, S.K., Bertino, M.F., 2010. Temperature dependent coercivity and magnetization of nickel ferrite nanoparticles. Journal of Magnetism and Magnetic Materials 322, 2199-2202. doi:10.1016/j.jmmm.2010.02.010

Ma, J., Chen, K., Zhang, X., 2012. Magnetic characteristics of $\mathrm{Fe}_{3} \mathrm{O}_{4} \alpha-\mathrm{Fe}_{2} \mathrm{O}_{3}$ hybrid cubes. Journal of Applied Physics 111, 033925-1-033925-6.

Malik, A.E., Hutchison, W.D., Nishimura, K., Elliman, R.G., 2012. Magnetic properties of $\mathrm{Co}, \mathrm{Ni}, \mathrm{Pt}$ and their alloy nanoparticles formed in $\mathrm{SiO}_{2}$ by ion beam synthesis. Nuclear Instrument and Methods in Physics Research Section B Beam Interactions with Materials and Atoms 272, 70-73. doi:10.1016/j.nimb.2011.01.035 
Ramappa, D.A., Henley, W.B., 1999. Diffusion of iron in silicon dioxide. Journal of The Electrochemical Society 146, 3773-3777.

Sprouster, D.J., Giulian, R., Araujo, L.L., Kluth, P., Johannessen, B., Kirby, N., Ridgway, M.C., 2011. Formation and structural characterization of $\mathrm{Ni}$ nanoparticles embedded in $\mathrm{SiO}_{2}$. Journal of Applied Physics 109, 113517. doi:10.1063/1.3594751

Swartzendruber, L., Itkin, V., Alcock, C., 1991. The Fe-Ni (iron-nickel) system. Journal of Phase Equilibria 12, 288-312.

Tejada, J., Martinez, B., Labarta, A., Chudnovsky, E.M., 1991. Correlated spin glass generated by structural disorder in the amorphous Dy6Fe74B20 alloy. Physical Review B 44, 7698.

Topkaya, R., Akman, ö., Kazan, S., Aktaş, B., Durmus, Z., Baykal, A., 2012. Surface spin disorder and spin-glass-like behaviour in manganesesubstituted cobalt ferrite nanoparticles. Journal of Nanoparticle Research 14. doi:10.1007/s11051-012-1156-2

Vitta, S., Khuntia, A., Ravikumar, G., Bahadur, D., 2008. Electrical and magnetic properties of nanocrystalline Fe100-xNix alloys. Journal of Magnetism and Magnetic Materials 320, 182-189.

Westerstrand, B., Nordblad, P., Nordborg, L., 1975. The magnetocrystalline anisotropy constants of iron and iron-silicon alloys. Physica Scripta 11, 383.

S. Xuan, Y.-X. J. Wang, J. C. Yu, and K. Cham-Fai Leung, Tuning the grain size and particle size of superparamagnetic $\mathrm{Fe}_{3} \mathrm{O}_{4}$ microparticles. Chemistry of Materials 21, 5079-5087, 2009.

Yuan, C.W., Yi, D.O., Sharp, I.D., Shin, S.J., Liao, C.Y., Guzman, J., Ager, J.W., Haller, E.E., Chrzan, D.C., 2009. Size-distribution evolution of ion-beamsynthesized nanoclusters in silica. Physical Review B 80. doi:10.1103/PhysRevB.80.134121

Yu, P., Jin, X.F., Kudrnovský, J., Wang, D.S., Bruno, P., 2008. Curie temperatures of fcc and bcc nickel and permalloy: Supercell and Green's function methods. Physical Review B 77. doi:10.1103/PhysRevB.77.054431

Zhang, D., Klabunde, K.J., Sorensen, C.M., Hadjipanayis, G.C., 1998. Magnetization temperature dependence in iron nanoparticles. Physical Review B 58, 14167.

Zhang, B., Fenineche, N.-E., Liao, H., Coddet, C., 2013. Magnetic properties of in-situ synthesized $\mathrm{FeNi}_{3}$ by selective laser melting $\mathrm{Fe}-80 \% \mathrm{Ni}$ powders. Journal of Magnetism and Magnetic Materials 336, 49-54. doi:10.1016/j.jmmm.2013.02.014

Q. T. Zhang, L. You, C. H. Wan, Z. H. Yuan, X. Zhang, J. Wang, and X. F. Han, Influence of epitaxial $\mathrm{BiFeO}_{3}$ on superparamagnetic behavior of $\mathrm{CoFeB}$ thin film, Journal of Applied Physics, vol. 117, no. 14, p. 143904, Apr. 2015. 
A. Zeleňáková, V. Zeleňák, J. Bednarčík, P. Hrubovčák, and J. Kováč, 2014. Magnetic nanocomposites of periodic mesoporous silica: The influence of the silica substrate dimensionality on the inter-particle magnetic interactions, Journal of Alloys and Compounds, 582, 483-490.

Zuhr, R.A., Magruder, R.H., Anderson, T.S., 1998. Formation and optical properties of intermetallic nanoclusters formed by sequential ion implantation. Surface Coating Technology 103, 401-408. 


\section{Chapter 7}

\section{Summary}

This thesis reports the results from a study of new methods to make magnetic nanoparticles. Specifically, permalloy and magnetite powders containing nanoparticles were made by a new arc-discharge method and the magnetite results were compared with those from magnetite nanoparticles made by a chemical process. $\mathrm{Ni}_{1-\mathrm{x}} \mathrm{Fe}_{\mathrm{x}}$ nanoparticles were made for the first time by low energy dual ion implantation. Structural, magnetic and transport measurements were used to understand the synthesised compounds. The key results are summarized below followed by the outlook for further work.

\subsection{Structural and vibrational studies}

It was shown that the new arc-discharge method could be used to make P78 and P45 permalloy and magnetite powders containing nanoparticles with sizes as small as $7 \mathrm{~nm}$ and particle sizes up to $25 \mu \mathrm{m}$. Raman measurements on the magnetite powders showed that there was also some maghemite and hematite. Magnetite nanoparticles were made by a co-precipitation method with average diameters of $14 \mathrm{~nm}$, where Raman measurements showed that there is also some maghemite.

Low energy dual $\mathrm{Ni}$ and $\mathrm{Fe}$ implantation was done into a $\mathrm{SiO}_{2}$ film for the first time to create $\mathrm{Ni}_{\mathrm{x}} \mathrm{Fe}_{1-\mathrm{x}}$ nanoparticles. Implantation with $\mathrm{x}=0.82$ and $\mathrm{x}=0.45$ with the same $\mathrm{Ni}$ fluence resulted in an implantation layer with similar nanoparticle sizes where the average diameter $\sim 4 \mathrm{~nm}$. Electron beam annealing resulted in different nanoparticle size distributions. In particular, there was a bimodal particle size distribution for $\mathrm{x}=0.82$ with large $40 \mathrm{~nm}$ to $75 \mathrm{~nm}$ nearly symmetric nanoparticles at the surface and smaller $\sim 10 \mathrm{~nm}$ diameter nanoparticles extending further into the film. This can be contrasted with $\mathrm{x}=0.45$ where there 
were highly asymmetric $\mathrm{Ni}_{\mathrm{x}} \mathrm{Fe}_{1-\mathrm{x}}$ nanoparticles at the surface with sizes of $\sim 72 \mathrm{~nm}$ to $140 \mathrm{~nm}$ and very few smaller $\mathrm{Ni}_{\mathrm{x}} \mathrm{Fe}_{1-\mathrm{x}}$ nanoparticles. Some of the $\mathrm{Ni}_{\mathrm{x}} \mathrm{Fe}_{1-\mathrm{x}}$ nanoparticles were also surrounded by a $\mathrm{FeSi}_{\mathrm{y}} \mathrm{O}_{\mathrm{x}}$ shell. The different nanoparticle size distributions and morphologies are likely to be related to the existence of $\mathrm{Ni}_{\mathrm{x}} \mathrm{Fe}_{1-\mathrm{x}}$ nanoparticles in the as-implanted films that act as nucleation centres as well as different initial $\mathrm{Ni}$ and Fe depth concentrations.

\subsection{Magnetic study}

The magnetite powder made by arc-discharge had a saturation magnetization that was less than the expected value due to the presence of some antiferromagnetic hematite. The value is also reduced in magnetite nanoparticles made by the co-precipitation method due to spin-disordered nanoparticle shells. The P78 and P45 permalloy powders made by arc-discharge also had lower saturation moments. It was comparable to that of the bulk for $\mathrm{Ni}_{\mathrm{x}} \mathrm{Fe}_{1-\mathrm{x}}$ with $\mathrm{x}=0.82$ after electron beam annealing and for $\mathrm{x}=0.53$ after implantation with a higher $\mathrm{Ni}$ fluence. Lower than expected values were found for $\mathrm{x}=0.45$ after EBA that may be due to formation of Fe-rich antiferromagnetic shells. It was also lower after implantation for $\mathrm{x}=0.78$ and $\mathrm{x}=0.45$, which is likely to be due to spin-disordered shells and some Ni and Fe ions that do not magnetically order. The contribution of spin glass effects at high fields was prominent in the smaller nanoparticles, i.e. the magnetite made by co-precipitation as well as the superparamagnetic nanoparticles formed upon implantation.

Superparamagnetism was observed for as-implanted $\mathrm{Ni}_{\mathrm{x}} \mathrm{Fe}_{1-\mathrm{x}}$ with $\mathrm{x}=0.45$, 0.53 and 0.78 , which was consistent with the small particle sizes. It was not observed after EBA because most of the nanoparticles were bigger than the superparamagnetic limit. The magnetite nanoparticles made by the coprecipitation method also had sizes $(\sim 14 \mathrm{~nm})$ that were larger than the superparamagnetic limit. There was no evidence for superparamagnetism in the 
permalloy or magnetite powders made by the arc-discharge method since most of the particle sizes were large.

The temperature dependence of the saturation magnetization was studied for all samples. It was found that the low temperature exponent of $\mathrm{M}_{\mathrm{s}}(\mathrm{T})$ was $3 / 2$ for magnetite made by arc-discharge. This is the same as that expected for a Heisenberg ferromagnet and it was also reported in a magnetite single crystal. It was found that $J_{a b} / k_{\mathrm{B}}=42 \mathrm{~K}$. A lower exponent was observed for the $\sim 14 \mathrm{~nm}$ diameter magnetite nanoparticles made by the co-precipitation method, which may be due to spin-disordered shells. There was a different exponent at higher temperatures above the Verwey transition that could be seen in the $\mathrm{M}_{\mathrm{s}}(\mathrm{T})$ data. By comparison with a previous study, it is evident that the Verwey transition disappears for magnetite nanoparticle diameters somewhere between $4 \mathrm{~nm}$ and 14 nm. As-implanted $\mathrm{Ni}_{\mathrm{x}} \mathrm{Fe}_{1-\mathrm{x}}$ with $\mathrm{x}=0.82$ had an exponent much less that the expected $3 / 2$. This is likely to be due to the morphology of the implanted region and magnon propagation between nanoparticles. The other implanted and annealed $\mathrm{Ni}_{\mathrm{x}} \mathrm{Fe}_{1-\mathrm{x}}$ nanoparticles all had a $\mathrm{M}_{\mathrm{s}}(\mathrm{T})$ that could be fitted with an exponent of $3 / 2$ as well as a contribution from a spin disordered shell that displayed spin-freezing at low temperatures.

The susceptibilities were highest for as-implanted $\mathrm{Ni}_{\mathrm{x}} \mathrm{Fe}_{1-\mathrm{x}}$ with a high $\mathrm{Ni}$ fluence and $\mathrm{x}=0.53$ where it reached 90 . It was $\sim 16$ for lower Ni fluences with $\mathrm{x}=0.45$ and $\mathrm{x}=0.78$ after EBA where the initial as-implanted values were as low as $\sim 5$. The susceptibility was also low for P78 and P45 made by arc-discharge where it was $\sim 11$. These are all less than that expected in the bulk and it likely to be related to demagnetization effects. There could also be a contribution from disorder that can lead to domain wall pining. The results show that using high $\mathrm{Ni}$ fluences is a method that can be used to maximize the permeability. 


\subsection{Magnetotransport study}

Magnetoresistance was observed in P78 and P45 pressed pellets that is due to the ordinary magnetoresistance and spin-dependent tunnelling. There was no evidence for an anisotropic MR from s-d scattering. The spin-dependent tunnelling MR was low due to spin-disorder in the grain boundary region. Spindependent tunnelling was also observed in magnetite made by arc-discharge and the co-precipitation method. It was much larger and reached $-9.5 \%$ at $120 \mathrm{~K}$ and high magnetic fields. The MR was similar for both magnetite preparation methods. A model was developed to explain the lower than expected MR values that included the effect of spin-disordered shells and grain boundaries. The behaviour of the MR at low fields was studied in the permalloy as well as magnetite pellets. There was a large coercivity compared to that of the magnetization in the permalloy as well as the magnetite. The similarity in both systems further confirm that the behaviour was because of conduction pathways through the grain boundaries through a highly spin polarized material. The electrical resistance for the P78 and P45 powders had the temperature dependence expected for a metal. This can be contrasted with the magnetite powders made by arc-discharge and the co-precipitation method. In this case the electrical resistance had an exponential dependence of $\mathrm{T}^{-1 / 2}$ that can be attributed to a Coulomb charging effect and tunnelling between nanoparticles. It occurs in the magnetite powders due to small nanoparticles coating the large particles. $\kappa_{0} / \mathrm{k}_{\mathrm{B}}$ is larger for the nanoparticles made by the co-precipitation method due to the smaller nanoparticle size and it is also possible that tunnelling barrier heights are different. 


\subsection{Outlook}

This section outlines further projects that could help to understand magnetic nanostructured materials and their properties. Additional measurements may include obtaining depth-dependant concentration profiles of the ion implanted films perhaps by high resolution RBS as well as probing the nanoparticle composition by X-ray photoelectron spectroscopy. It would also be interesting to do high resolution magnetic scanning to locally probe the nanoparticles made by ion implantation and annealing.

It would be useful to make $\mathrm{Ni}_{\mathrm{x}} \mathrm{Fe}_{1-\mathrm{x}}$ nanoparticle films with higher fluencies because this could lead to an enhancement of the susceptibility. EBA studies should also be performed for the same reason. Different $\mathrm{x}$ values with $\mathrm{x}$ between 0.45 and 0.78 should be studied and in particular $\mathrm{x} \sim 0.7$ where the current results suggest that only large $\mathrm{Ni}_{\mathrm{x}} \mathrm{Fe}_{1-\mathrm{x}}$ nanoparticle might be made. Higher implantation energies could be studied that may lead to different nanoparticle morphologies including the possibility of predominantly small nanoparticles that may be superparamagnetic.

The arc-discharge method should be modified to increase the fraction of magnetic nanoparticles. This could be done for example by making the modification discussed in section 3.2.4. A constant horizontal gas flow could also be used to get small particles. The anode and cathode could also be rotated and the powders produced by the high temperature arc could be quenched into a liquid that may also lead to smaller particles. Different gases, partial pressures, and arc currents should be explored to optimise the quality of the resultant powders.

The current results suggest that the magnetite Verwey transition disappears for nanoparticle diameters between $4 \mathrm{~nm}$ and $\sim 14 \mathrm{~nm}$. Thus, it would be interesting to study nanoparticle powders with intermediate sizes. It would also be useful to study $\mathrm{R}(\mathrm{T})$ as a function of nanoparticle size and separation to see how well the current model Coulomb charging model fits the data. Annealing 
studies on pressed pellets should be studied in the nanoparticle and arc-discharge powders to see if this can result in higher magnetoresistances. 